
VARTATION IN TREATMENT ANDEOLON-UP

FROM THE PATIENTS PERSRECAN

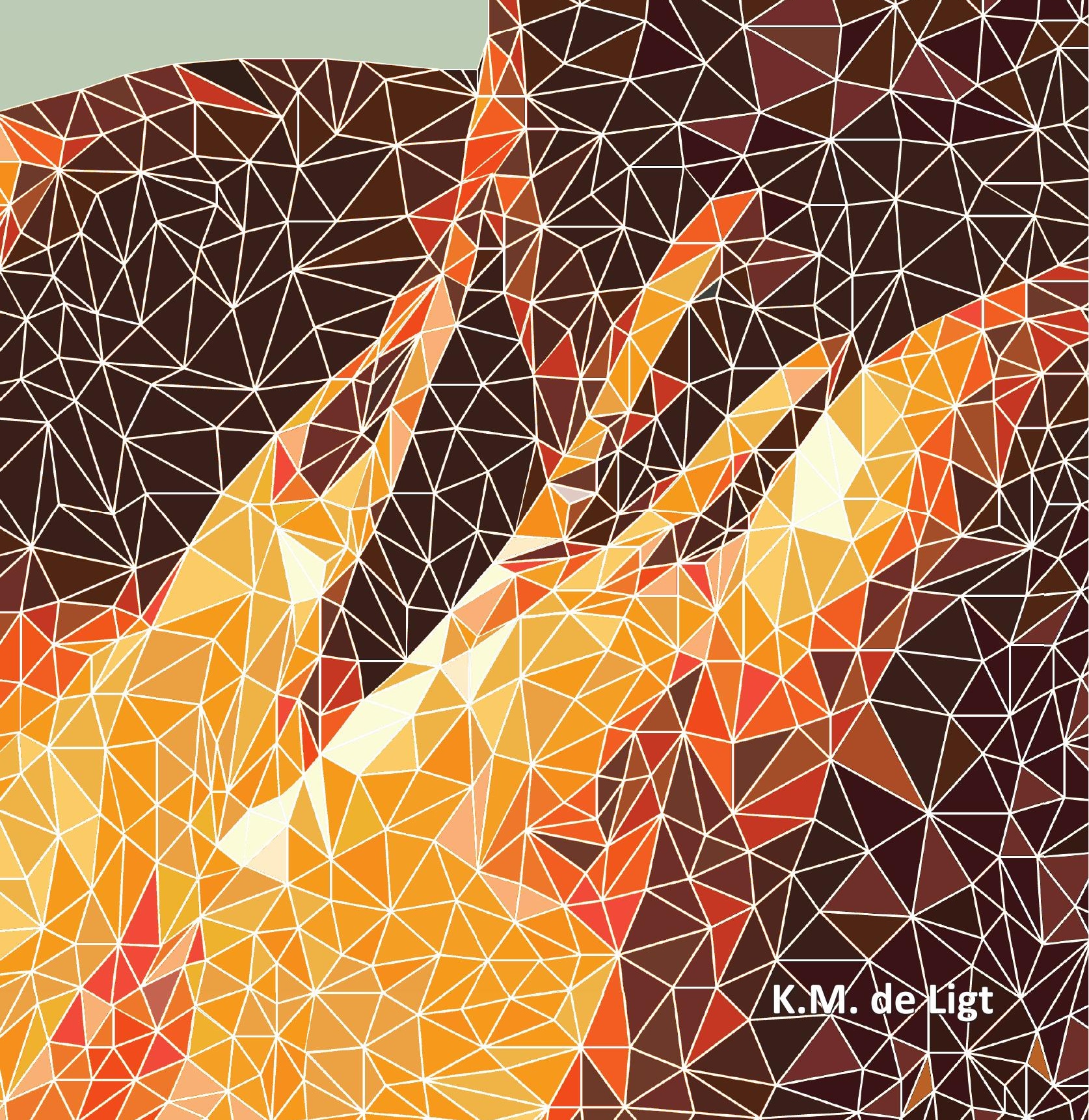



TOWARDS PERSONALISED BREAST CANCER CARE:

VARIATION IN TREATMENT AND FOLLOW-UP

FROM THE PATIENTS' PERSPECTIVE

Kelly de Ligt 



\title{
TOWARDS PERSONALISED BREAST CANCER CARE: VARIATION IN TREATMENT AND FOLLOW-UP FROM THE PATIENTS' PERSPECTIVE
}

\section{PROEFSCHRIFT}

\author{
ter verkrijging van \\ de graad van doctor aan de Universiteit Twente, \\ op gezag van de rector magnificus, \\ Prof. dr. T.T.M. Palstra, \\ volgens besluit van het College voor Promoties \\ in het openbaar te verdedigen \\ op vrijdag 13 september 2019 om 14.45 uur
}

door

\section{Kelly Maureen de Ligt}

geboren op 25 juni 1992

te Arnhem, Nederland 
Dit proefschrift is goedgekeurd door:

De promotor:

De copromotoren:
Prof. dr. S. Siesling

Dr. M.A.M. Mureau

Dr. C.H. Smorenburg

This thesis is part of the Health Science Series, HSS 19-28, department of Health Technology and Services Research, University of Twente, Enschede, the Netherlands. ISSN 1878-4968.

Financial support for printing of this thesis was kindly provided by:

\section{iKNL conedsesse

\section{UNIVERSITY OF TWENTE.}

Cover design and layout: Marlies van Hoof, madebymarlies.nl

Printed by: Ipskamp printing, Enschede

ISBN: 978-90-365-4815-1

DOI: $10.3990 / 1.9789036548151$

(C) Copyright 2019: Kelly de Ligt, Arnhem, the Netherlands. No parts of this thesis may be reproduced, stored in a retrieval system or transmitted in any form or by any means without permission of the author. Alle rechten voorbehouden. Niets uit deze uitgave mag worden vermenigvuldigd, in enige vorm of op enige wijze, zonder voorafgaande schriftelijke toestemming van de auteur. 
Promotiecommissie:

Voorzitter:

Secretaris:

Promotor:

Copromotoren:

Commissieleden:
Prof.dr. Th.A.J. Toonen, Universiteit Twente, BMS

Prof.dr. Th.A.J. Toonen, Universiteit Twente, BMS

Prof. dr. S. Siesling, Universiteit Twente

Dr. M.A.M. Mureau, Erasmus Medisch Centrum

Dr. C.H. Smorenburg, Nederlands Kanker Instituut Antoni van Leeuwenhoek

Prof. dr. W. H. van Harten, Universiteit Twente

Dr. C.H.C. Drossaert, Universiteit Twente

Prof. dr. L.V. van de Poll-Franse, Tilburg University

Prof. dr. J.A. Hazelzet, Erasmus Universiteit Rotterdam

Prof. dr. S.C. Linn, Universiteit Utrecht

Prof. dr. J.H.G. Klinkenbijl, Universiteit van Amsterdam 



\section{Table of contents}

General introduction and outline

First part Decisions about timing of chemotherapy

Chapter 1: Patients' experiences with decisions on timing of chemotherapy for breast cancer

Chapter 2: Current decisions on neoadjuvant chemotherapy for early breast cancer: experts' experiences in the Netherlands.

Second part Decisions about breast reconstruction

Chapter 3: Long-term health-related quality of life after four common surgical treatment options for breast cancer and the effect of complications a retrospective patient-reported survey among 1871 patients

Chapter 4: The effect of being informed on receiving immediate breast reconstruction in breast cancer patients

$\begin{array}{lll}\text { Third part } & \text { Potential for personalisation of follow-up } & 109\end{array}$

Chapter 5: Patient-reported health problems and healthcare use after treatment for early breast cancer

Chapter 6: The impact of health symptoms on health-related quality of life in earlystage breast cancer survivors

Chapter 7: Opportunities for personalised follow-up care among patients with breast cancer: A scoping review to identify preference-sensitive decisions

Summary, Conclusions and General discussion

List of publications 


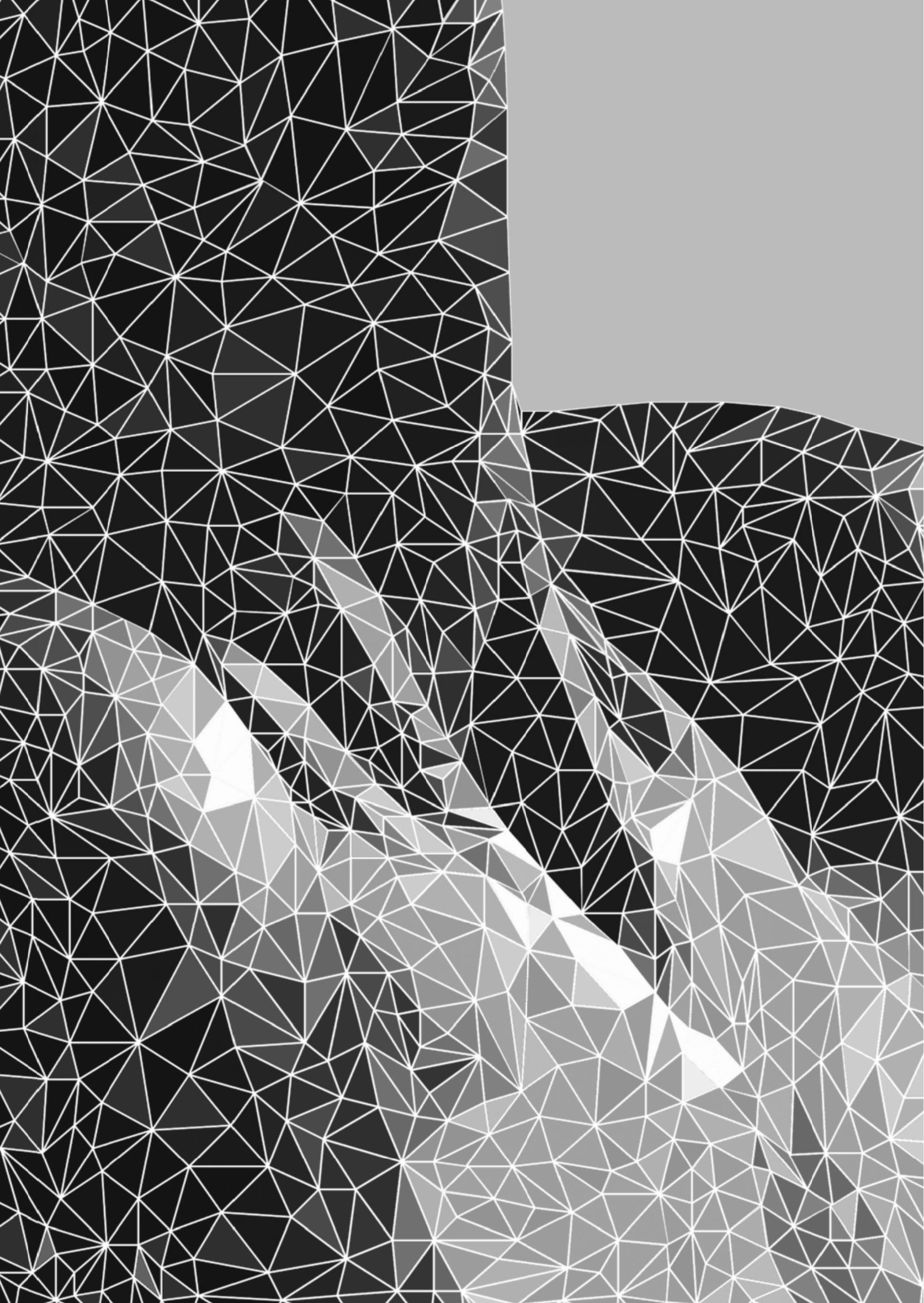


GENERAL INTRODUCTION AND OUTLINE

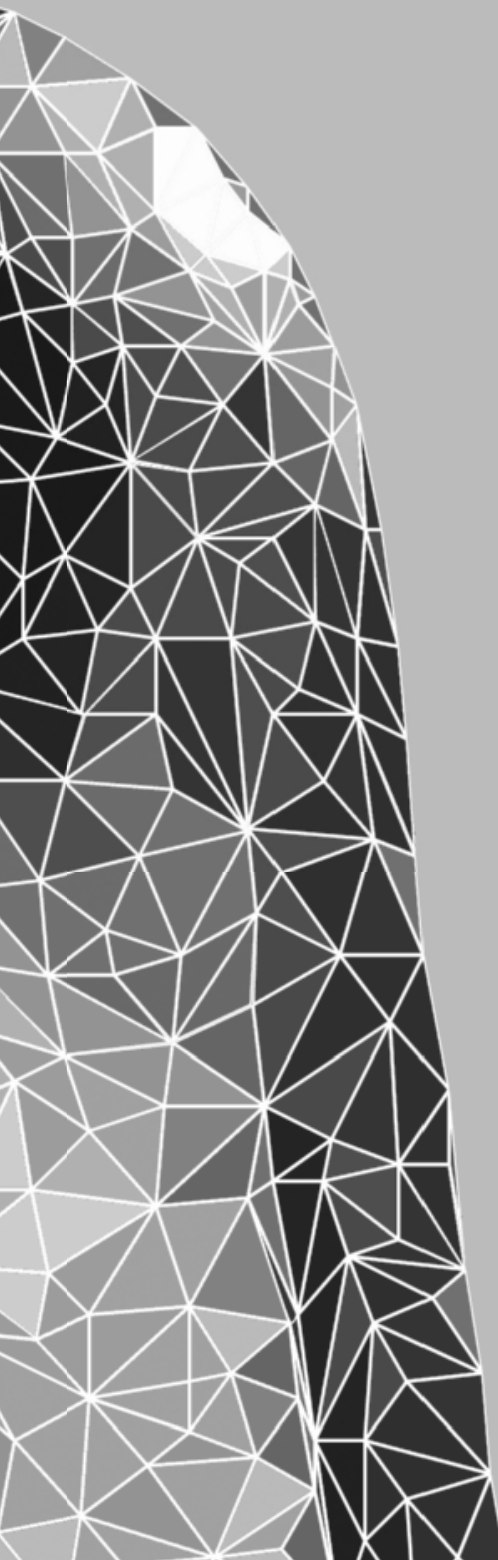





\section{General introduction and outline}

Breast cancer is the most commonly diagnosed cancer and the leading cause of cancer death in women worldwide [1]. At least one in eight women will develop breast cancer in her lifetime [2, 3]. In 2018, almost 2.1 million women worldwide were diagnosed with cancer [1]. Survival has been increasing over the past years, which is associated with a decrease in mortality through improved care and screening programmes [4]. Despite being the leading cause of cancer death in women worldwide, breast cancer patients in North America and Europe have a five-year survival over $80 \%$ [4], with rates exceeding $96 \%$ for stage I and $86 \%$ for stage II disease $[5,6]$. In the Netherlands, the 10 -year prevalence of invasive breast cancer was about 135.000 women in 2018 [7].

In general, non-metastasized breast cancer is treated by local surgical intervention. Surgery for breast cancer either consist of breast conserving surgery, in which the contour of the breast is spared, or mastectomy, in which all breast tissue is removed. Following mastectomy, a breast reconstruction may be performed to restore the contour of the breast. In addition to surgery, regional or locoregional radiotherapy, and/or systemic therapies such as chemotherapy, antihormonal therapy, or targeted therapy may be indicated to improve local control and survival. These treatment modalities are either administered before (i.e. neo-adjuvant) or after (i.e. adjuvant) surgical treatment. Primary treatment is followed by a minimum of five years of followup care, consisting of surveillance for recurrent disease, management of late and/or side-effects of treatment and attention for psychosocial effects, and evaluation of the care received by the patient. Other goals of follow-up care are the promotion of health and lifestyle and the management of anti-hormonal therapy, including therapy adherence and side effects [8-11].

Recommendations for diagnosis, treatment, and follow-up care for breast cancer are presented in international breast cancer guidelines [8-11]. In the Netherlands, the national evidence-based guideline for breast cancer has been developed by the multidisciplinary working group on breast cancer (Nationaal Borstkanker Overleg Nederland, NABON), and its modules are revised regularly to include relevant new insights [11]. Specifically for breast reconstruction, The Dutch Breast Reconstruction Guideline was developed to promote evidence-based choices concerning breast reconstruction options after mastectomy and breast conserving surgery [12]. Additionally to the follow-up recommendations in the breast cancer guideline, a guideline on cancer survivorship care in general ('Herstel na kanker') was developed to further support the trajectory of care after cancer treatment [13].

Cancer and cancer treatment can negatively affect the physical, psychological, social, and spiritual well-being of patients who are living with cancer or have survived cancer [14, 15]. Therefore, a patient-specific fit between patient and disease characteristics and the proposed therapy would be appropriate. Ideally, in treating cancer, overtreatment as well as undertreatment should be avoided, by choosing the least invasive therapy as possible, while maintaining optimal cancer control. By overtreatment, patients receive more aggressive treatment than necessary, possibly leading to needless side effects; by undertreatment, non-optimal cancer control may lead to increased risk of extended or recurrent disease $[16,17]$. We increasingly understand which specific treatment 
modalities are successful for which sub-groups of patients. With this knowledge, more personalized treatment of breast cancer can be achieved. Furthermore, predictive tools as 'MammaPrint' can be used to respectively estimate which patients really do require chemotherapy [18, 19]. Similar tools were developed as well to predict the benefit of radiotherapy [20]. After treatment, prognoses for overall and breast cancer specific survival can be calculated by the 'PREDICT' prognostic model [21], and the risk of locoregional recurrent cancer by the 'INFLUENCE' nomogram [22], which may guide personalized surveillance of recurrent disease. This way of personalized care, based on risks of a future event, is primarily based on clinical information, including patient characteristics (e.g. age), tumour characteristics (e.g. stage of disease, receptor status), and treatment characteristics (e.g type and extent of treatment). However, over the recent years, there has been a shift from a more generic and paternalistic approach of care towards a more patient-centred approach of care. Patient-centred care is care that is respectful and responsive to the preferences, needs, and value of individual patients, ensuring that all clinical decisions are guided by these aspects. With patient-centred care, cancer care has become more and more focused on the individual needs of cancer patients, both in clinical as in personal values [23].

Individual patients have their own context - the living conditions and environment they live in [24]. In general, a treatment decision will be based on the treatment option that yields a clear clinical or survival benefit over other possible options. However, treatment decisions without such predominant treatment option, in which the final decision may not be solely based on clinical reasoning, are of a less straightforward nature. These decisions are called preference-sensitive decisions. They are not merely based on a trade-off between risks and benefits based on all relevant available evidence, but also incorporate patient preferences. These decisions occur when several treatment options may lead to similar results in terms of survival, or are equally desirable in terms of clinical outcomes. Or, evidence about the risks of options may be lacking, regardless of the possible benefits the particular option may yield, leading to a trade-off in uncertainty. Last, the efforts the patient must make to adhere to the best option from a clinical perspective can be high or severely intervene with the patient's lifestyle, requesting a certain effort or dedication from the patient towards this treatment option. Thus, treatment recommendations do not only require diagnosis of the medical condition, but of the patient preferences as well [25]. Decisionmaking is then the result of the patient and physician working together to include the best available evidence, the patient's context and preferences, and the physicians clinical advice [26]. This collaboration in decision-making is called 'shared decision-making'. In order for a breast cancer patient to make an informed decision, she needs to be aware that there is a decision to be made, and she needs to receive the most up-to-date evidence-based information about the risks and benefits of each treatment option within this decision. Subsequently, the physician's advice and the patient's preferences are both incorporated in the final treatment decision $[27,28]$

When personalised care is provided, i.e. care that is in line with the patients characteristics and preferences, this may cause treatment variation. However, this is then a preferable result of personalized care processes. Variation can emerge in various aspects of care, for instance by decisions between treatment modalities, treatment timing, or sequence of treatment options. However, variation in care may also be the result of a lack of quality of care or access to treatment 
options, which is regarded undesirable variation. The underlying cause of variation may then be a result of unfavourable hospital characteristics, or inadequate processes of care. In order to determine whether variation in treatment administration is undesirable, and is caused by certain patient, tumour, treatment, or hospital characteristics, it is important to investigate all factors that might cause this variation, including the underlying process of decision-making. As some causes of unwanted variation may be reversible, observed treatment variation may be a starting point for quality of care improvements.

Within the development towards personalised or patient-centred care and shared decision-making, the treatment outcomes and experiences as assessed by the patient have become important outcomes, in addition to clinical treatment outcomes $[29,30]$. These experiences can be measured through validated questionnaires: Patient-Reported Outcome Measures (PROMs). PROMs measure the patient's functional status and health-related quality of life, including the patient's view on potentially experienced symptoms. PROMs are targeted at either the population in general or at a diseased population in specific. The first are more applicable to general aspects of health and wellbeing, while the latter are used in comparisons across conditions [29, 31]. Targeted to a specific patient population, such as patients with cancer, are cancer-specific PROMs such as the EORTCQLQ-C30, breast cancer-specific such as the EORTC-QLQ-BR23/45, or treatment-specific such as the Breast- $Q$ for patients treated with breast surgery [32]. Furthermore, questionnaires can be targeted at patient satisfaction and patient experiences with a health service or healthcare. PatientReported Experience Measures (PREMs) are generic tools that can be used in wider populations and non-specific settings, and report the quality of care from a patient's perspective [29].

The use of PROMs may have multiple goals. First, physicians may use PROMs as a means to assess the patient's health status, aiming to detect possible health problems that may require further attention. PROM results are then discussed during outpatient consultations. Second, PROMs may be applied for research: routine PROM assessments can provide a reflection of every-day care practice, giving insight in the effectiveness of care. Last, they may be used by patients to determine quality of care, and by providers to improve quality of care, as insight is gained in the institution's performance [31]. Valuable care is reached through increasing safety, quality, patient-centeredness, timeliness, efficiency, and equitability, as described in the earlier work of 'Crossing the Quality Chasm' by the Committee on Quality of Health Care in America [23]. Overall, PROMs have the potential to transform health care, i.e. to improve quality and safety through patient-centred provision of care, which has been recognized in the recent years [29, 31]. This potential is the foundation of Porter's Value Based Health Care (VBHC). Within VBHC, the goal is to maximize the value of care from the patients' perspective in relation to the costs of providing this care. Porter defined that outcomes should include the health circumstances that are most valuable to the patient [33]. Towards the measurement of values and preferences of patients, PROMs can be used to measure what patients value and where value could be added [31]. The Dutch government stimulates the implementation of VBHC as a means to promote the quality of care, the patients quality of life, and the physicians work satisfaction, and published a report in which the plans and development towards VBHC for the upcoming years were set forth [34]. 
This thesis describes variation in care for breast cancer patients in the Netherlands. The objective was to investigate whether patient preferences, needs, experiences, outcomes, and shared decision-making can explain the variation observed within three treatment modalities for breast cancer in the Netherlands. The three treatment modalities and subjects of treatment-decisions highlighted in this thesis are the timing of chemotherapy treatment, the application of breast reconstruction, and decisions about follow-up care. Patient-reported outcomes and experiences were the main source of information towards this goal, putting the patients' perspective at the heart of this thesis.

The first part deals with the variation in the timing of chemotherapy treatment for stage II and III breast cancer, either prior to or following breast surgery. Originally, neoadjuvant chemotherapy was indicated to enable surgery in inoperable breast cancer, but current use in operable breast cancer aims to enable breast conserving surgery and axilla preserving surgery, and to assess treatment response [35]. Despite these potential advantages over adjuvant timing of chemotherapy, use of neoadjuvant chemotherapy was lower than for adjuvant chemotherapy and varied among hospitals [36]. As not all variation could be explained by tumour and patient characteristics [37], it was hypothesized that information provision was an underlying factor for this variation. In Chapter 1, patients' experiences with the timing of chemotherapy are described following from survey results of almost 400 patients. Indications for and beliefs about treatment from almost 140 surgeons and oncologists in the Netherlands are described in Chapter $\mathbf{2}$ to further unfold possible causes for the observed variation. By that, this part is a diptych of the patients' and physicians' perspective of chemotherapy timing.

The second part of this thesis discusses surgical treatment options for breast cancer patients with a focus on the use of breast reconstruction in patients who underwent mastectomy. In Chapter 3 the importance of breast reconstruction and preservation is quantified by measuring quality of life after breast cancer surgery. Breast reconstruction yields positive effects on psychosocial outcomes and quality of life [38,39], but is elective within the treatment of breast cancer. It can be performed either at the time of breast cancer surgery (immediate breast reconstruction, IBR) or afterwards (delayed breast reconstruction, DBR) [38]. Specifically for IBR, considerable variation was observed between hospitals in the Netherlands. As this could not be fully explained by tumour, patient, and hospital factors [40,41], in Chapter 4 it was investigated whether the process of information provision and decision-making contributed to the observed variation in IBR.

Within the third part of this thesis, follow-up care for breast cancer patients is discussed. It was hypothesized that current follow-up for breast cancer patients does not (optimally) meet the individual patient's needs, suggesting further personalization may be preferred [42-45]. One aim of follow up care is to detect and manage effects of treatment [8-10]. Variation is expected in the prevalence and severity of these treatment-related health problems and associated use of care. Chapter 5 aims to determine treatment-related health symptoms and use of care in breast cancer patients up to five years after diagnosis, and in Chapter 6, the association between these symptoms and health-related quality of life in breast cancer patients up to five years after diagnosis is investigated. To move towards more personalized follow-up care, Chapter 7 describes which 
preference-sensitive decisions about common health problems and issues occur during followup, and to what degree shared decision-making is applied to provide care in line with patient preferences 


\section{References}

1. Bray F, Ferlay J, Soerjomataram I, Siegel RL, Torre LA, Jemal A. Global Cancer Statistics 2018: GLOBOCAN Estimates of Incidence and Mortality Worldwide for 36 Cancers in 185 Countries. CA: A Cancer Journal for Clinicians. 2018;0:1-31.

2. van der Waal D, Verbeek ALM, den Heeten GJ, Ripping TM, Tjan-Heijnen VCG, Broeders MJM. Breast cancer diagnosis and death in The Netherlands: a changing burden. European Journal of Public Health. 2011;25(2):320-4.

3. Wise J. Number of UK women who will get breast cancer has risen to one in eight. British Medical Journal. 2011;342:d808.

4. Coleman MP, Quaresma M, Berrino F, Lutz JM, De Angelis R, Capocaccia R, et al. Cancer survival in five continents: a worldwide population-based study (CONCORD). Lancet Oncology. 2008;9:730-56.

5. Janssen-Heijnen MLG, van Steenbergen LN, Voogd AC, Tjan-Heijnen VCG, Nijhuis PH, Poortmans PM, et al. Small but significant excess mortality compared with the general population for long-term survivors of breast cancer in the Netherlands. Annals of Oncology. 2014;25:64-8.

6. Howlader N, Noone AM, Krapcho M, Miller D, Bishop K, Kosary CL, et al. SEER Cancer Statistics Review, 1975-2014 Bethesda, MD: National Cancer Institute; 2017 [updated Based on November 2016 SEER data submission, posted to the SEER web site April 2017. Available from: https://seer.cancer.gov/csr/1975_2014/.

7. Netherlands Comprehensive Cancer Organisation (IKNL). Prevalence: Netherlands Comprehensive Cancer Organisation (IKNL),,; 2018 [Available from: https://www.cijfersoverkanker.nl/selecties/dataset_1/ img5bb31f884e973.

8. Senkus E, Kyriakides S, Ohno S, Penault-Llorca F, Poortmans P, Rutgers E, et al. Primary breast cancer: ESMO Clinical Practice Guidelines for diagnosis, treatment and follow-up. Annals of Oncology. 2015;26:8-30.

9. Runowicz CD, Leach CR, Henry NL, Henry KS, Mackey HT, Cowens-Alvarado RL, et al. American Cancer Society/American Society of Clinical Oncology Breast Cancer Survivorship Care Guideline. Journal of Clinical Oncology. 2016;34(6):611-35.

10. National Institute for Health and Clinical Excellence. Early and locally advanced breast cancer: diagnosis and treatment, NICE guideline. National Collaborating Centre for Cancer; 2009.

11. Netherlands Comprehensive Cancer Organisation (IKNL). National guideline on Breast Cancer. Netherlands Comprehensive Cancer Organisation (IKNL),,; 2012.

12. Mureau MAM, Nederlandse Vereniging voor Plastische Chirurgie (NVPC). Dutch Breast Reconstruction Guideline. Journal of Plastic Reconstructive and Aesthetic Surgery 2018:290-304.

13. Netherlands Comprehensive Cancer Organisation (IKNL). Guideline on Cancer Survivorship Care. 2011.

14. Ewertz $M$, Jensen $A B$. Late effects of breast cancer treatment and potentials for rehabilitation. Acta Oncologica. 2011;50:187-93.

15. Mayer DK, Nasso SF, Earp JA. Defining cancer survivors, their needs, and perspectives on survivorship health care in the USA. The Lancet. 2017;18(1):e11-e8.

16. Katz SJ, Morrow M. Addressing Overtreatment in Breast Cancer: The Doctors' Dilemma. Cancer. 2013;119(20):3584-8.

17. MacNeill F, Karakatsanis A. Over surgery in breast cancer. The Breast. 2017;31:284-9.

18. Ravdin PM, Siminoff LA, Davis GJ, Mercer MB, Hewlett J, Gerson N, et al. Computer Program to Assist in Making Decisions About Adjuvant Therapy for Women With Early Breast Cancer. Journal of Clinical Oncology. 2001;19(4):980-91.

19. Cardoso F, van't Veer LJ, Bogaerts J, Slaets L, Viale G, Delaloge S, et al. 70-Gene Signature as an Aid to Treatment Decisions in Early-Stage Breast Cancer. The New England Journal of Medicine. 2016;375(8):71729.

20. Chen K, Su F, Jacobs LK. A Nomogram to Predict the Benefit of Radiation Therapy After Breast-Conserving Surgery in Elderly Patients with Stage I \& ER-Negative, or Stage II/III Disease. Annals of Surgical Oncology. 2015;22(11):3497-503. 
21. Wishart GC, Azzato EM, Greenberg DC, Rashbass J, Kearins O, Lawrence G, et al. PREDICT: a new UK prognostic model that predicts survival following surgery for invasive breast cancer. Breast Cancer Research. 2010;12(R1).

22. Witteveen A, Vliegen IMH, Sonke GS, Klaase JM, IJzerman MJ, Siesling S. Personalisation of breast cancer follow-up: a time-dependent prognostic nomogram for the estimation of annual risk of locoregional recurrence in early breast cancer patients. Breast Cancer Research and Treatment. 2015;152(3):627-36.

23. Committee on Quality of Health Care in America Institute of Medicine (US). Crossing the Quality Chasm: A New Health System for the 21st Century.: National Academy Press; 2001.

24. The Council for Public Health and Society. No evidence without context: About the illusion of evidencebased practice in heatchcare. Den Haag2017.

25. Mulley AG, Chris T, Elwyn G. Stop the silent misdiagnosis: patients' preferences matter. British Medical Journal. 2012;245:e6572.

26. van der Weijden T, Pieterse AH, Koelewijn-van Loon MS, Knaapen L, Légaré F, Boivin A, et al. How can clinical practice guidelines be adapted to facilitate shared decision making? A qualitative key-informant study. BMJ Quality \& Safety. 2013;22:855-63.

27. Légaré F, Witteman HO. Shared Decision Making: Examining Key Elements And Barriers To Adoption Into Routine Clinical Practice. Health Affairs. 2013;32(2):276-84.

28. Stiggelbout AM, Pieterse AH, De Haes JCJM. Shared decision making: Concepts, evidence, and practice. Patient Education and Counseling. 2015;98:1172-9.

29. Weldring T, Smith SMS. Patient-Reported Outcomes (Pro's) and Patient-Reported Outcome Measures (Prom's). Health Services Insights. 2013;6:61-8.

30. Cribb A, Owens J. Whatever suits you: unpicking personalization for the NHS. Journal of Evaluation in Clinical Practise. 2010;16:310-4.

31. Black N. Patient reported outcome measures could help transform healthcare. British Medical Journal. 2013;346:f167.

32. Ong WL, Schouwenburg MG, van Bommel ACM, Stowell C, Allison KH, Benn KE, et al. A Standard Set of ValueBased Patient-Centered Outcomes for Breast Cancer: The International Consortium for Health Outcomes Measurement (ICHOM) Initiative. JAMA Oncology. 2017;3(5):677-85.

33. Porter ME. What Is Value in Health Care? New England Journal of Medicine 2010;363:2477-81.

34. Rijksoverheid. Ontwikkeling Uitkomstgerichte zorg 2018-2022. In: Ministerie van Volksgezondheid Welzijn en Sport, editor. 2018.

35. Mieog JS, van der Hage JA, van de Velde CJ. Preoperative chemotherapy for women with operable breast cancer. Cochrane Database Systematic Review. 2007;CD005002.

36. Dutch Institute for Clinical Auditing. Jaarrapportage 2013. NABON Breast Cancer Audit; 2014.

37. Spronk PER, van Bommel ACM, Vrancken Peeters MTFD, Siesling S, Smorenburg CH. Variation in the use of neoadjuvant chemotherapy for stage III breast cancer: results of the Dutch nationwide breast cancer registry NBCA (Nabon Breast Cancer Audit). The Breast. 2017;36:34-8.

38. Cordeiro PG. Breast Reconstruction after Surgery for Breast Cancer. New England Journal of Medicine. 2008(359):1590-601.

39. Tondu T, Tjalma WAA, Thiessen FEF. Breast reconstruction after mastectomy. European Journal of Obstetrics \& Gynecology and Reproductive Biology. 2018;article in press.

40. van Bommel ACM, Mureau MAM, Schreuder K, van Dalen T, Vrancken Peeters MTFD, Schrieks M, et al. Large variation between hospitals in immediate breast reconstruction rates after mastectomy for breast cancer in the Netherlands. Journal of Plastic, Reconstructive \& Aesthetic Surgery. 2017;70(2):215-21.

41. Schreuder K, Van Bommel ACM, De Ligt KM, Maduro JH, Vrancken Peeters MTFD, Mureau MAM, et al. Hospital organizational factors affect the use of immediate breast reconstruction after mastectomy for breast cancer in the Netherlands. Breast (Edinburgh, Scotland). 2017;34:96-102.

42. DH Macmillan Cancer Support, NHS Improvement. National Cancer Survivorship Initiative: Vision. 2010.

43. Donnelly P, Hiller L, Bathers S, Bowden S, Coleman R. Questioning specialists' attitudes to breast cancer follow-up in primary care. Annals of Oncology. 2007;18:1467-76. 
44. Montgomery DA, Krupa K, Cooke TG. Alternative methods of follow up in breast cancer: a systematic review of the literature. British Journal of Cancer. 2007;96:1625-32.

45. Zorginstituut Nederland. Verbetersignalement Zinnige nacontrole bij vrouwen behandeld voor borstkanker. Diemen: Zorginstituut Nederland; 2016. 



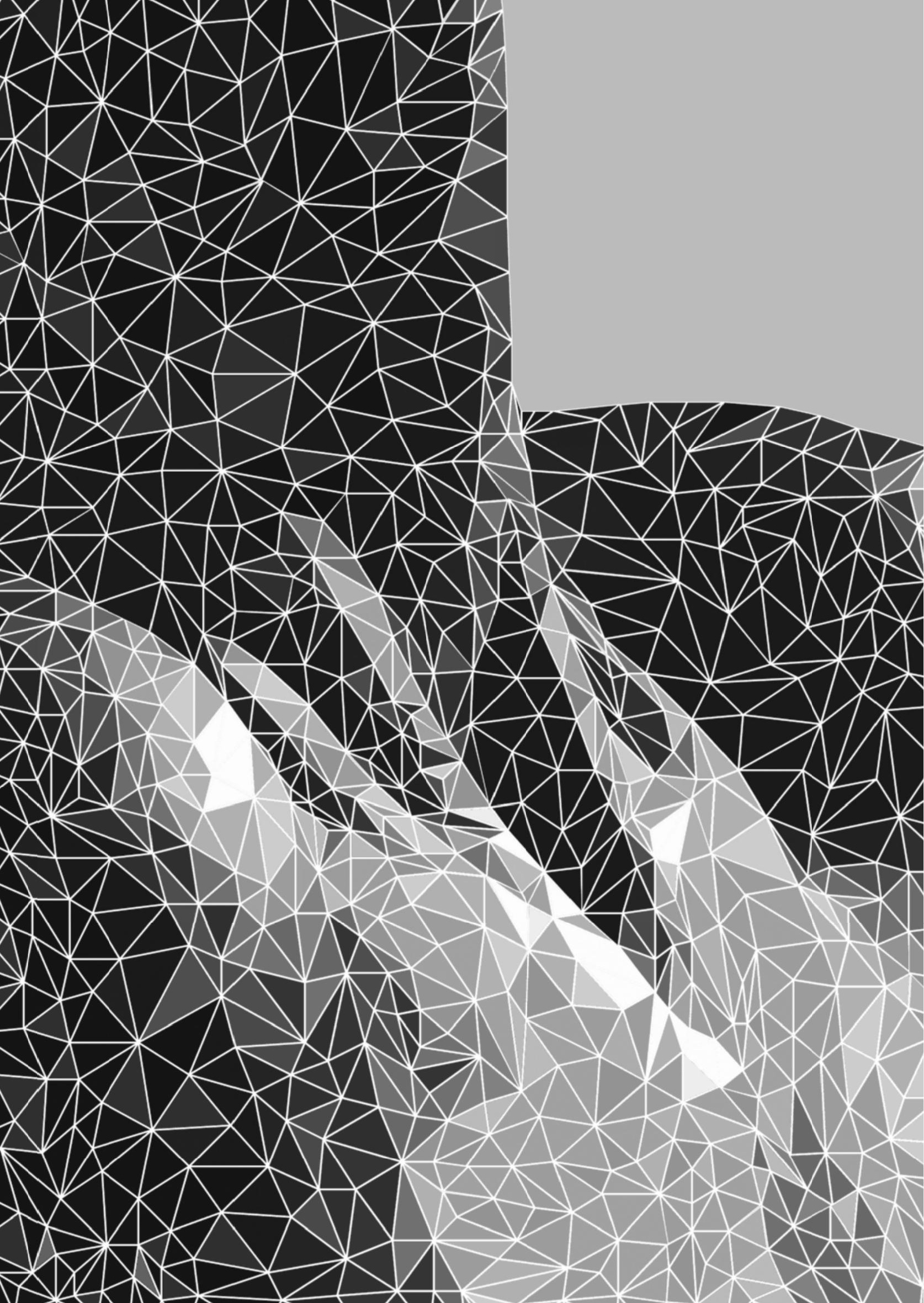


FIRST PART

DECISIONS ABOUT TIMING OF CHEMOTHERAPY 


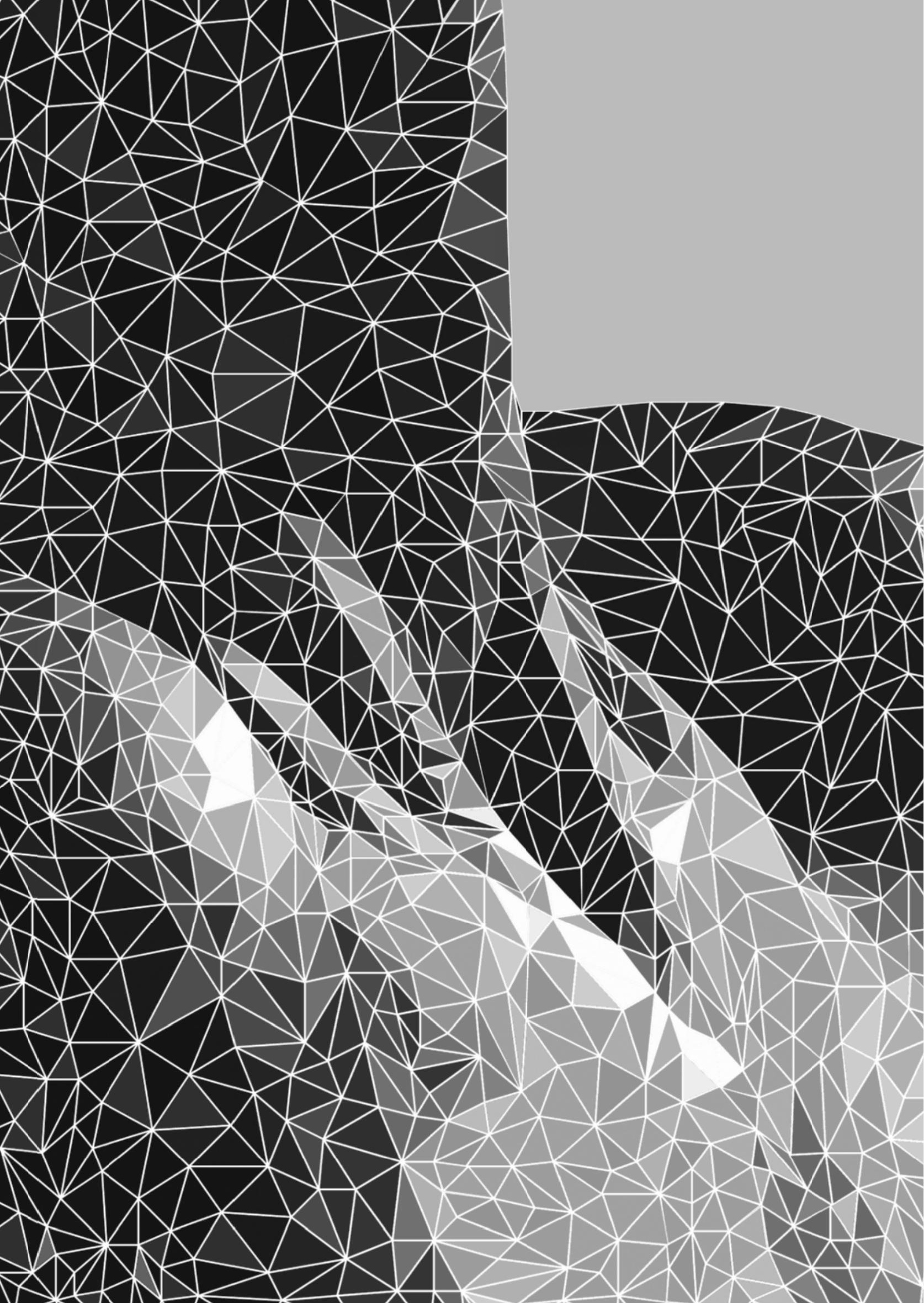


PATIENTS' EXPERIENCES WITH DECISIONS ON TIMING OF CHEMOTHERAPY FOR BREAST CANCER

K.M. de Ligt*

P.E.R. Spronk*

A.C.M. van Bommel

M.T.F.D. Vrancken Peeters

S. Siesling

C.H. Smorenburg

- on behalf of the NABON Breast Cancer Audit group -

* contributed equally

Breast. 2018 Feb;37:99-106. 



\section{Abstract}

Introduction:

Despite potential advantages, application of chemotherapy in the neo-adjuvant (NAC) instead of adjuvant (AC) setting for breast cancer (BC) patients varies among hospitals. The aim of this study was to gain insight in patients' experiences with decisions on the timing of chemotherapy for stage II and III BC.

\section{Materials and methods:}

A 35-item online questionnaire was distributed among female patients (age $>18$ ) treated with either NAC or AC for clinical stage II/III invasive BC in 2013-2014 in the Netherlands. Outcome measures were the experienced exchange of information on the possible choice between both options and patients' involvement in the final decision on chemotherapy timing. Chemotherapy treatment experience was measured with the Cancer Therapy Satisfaction Questionnaire (CTSQ).

\section{Results:}

Of 805 invited patients, 49\% responded (179 NAC, 215 AC). NAC-treated patients were younger and more often treated in teaching/academic hospitals and high-volume hospitals. Information on the possibility of NAC was given to a minority of AC-treated patients ( AC, stage II: $14 \%$, stage III: 31\%). Information on pros and cons of both NAC and AC was rated sufficient in about three fourth of respondents. Respondents not always felt having a choice in the timing of chemotherapy (stage II: $54 \%$ NAC vs $36 \%$ AC; stage III: $26 \%$ NAC, $54 \%$ AC).

\section{Conclusion:}

The need to make a treatment decision on NAC was found to be made explicit in only a small number of adjuvant treated patients, in particular in BC stage II. Less than half of the respondents felt they had a real choice. 


\section{Introduction}

Breast cancer $(\mathrm{BC})$ care consists of a multidisciplinary approach of surgery, radiation, and systemic therapy including chemotherapy [1]. Chemotherapy intents to eliminate potential existing micrometastases, thus decreasing recurrence rates and mortality [2]; it is timed either prior to or following surgery, respectively neoadjuvant (NAC) or adjuvant (AC), both leading to similar disease free and overall survival $[1,3,4]$. NAC versus AC yields several advantages. Down-staging of the primary tumour increases resectability and the possibility of breast conserving surgery (BCS) [4] and axillary preserving surgery [5]. Moreover, the response to chemotherapy can be assessed [1, $3,4,6]$, creating a platform to study the activity of (novel) agents or therapeutic combinations in a patient-personalized way $[3,4,7,8]$.

(Inter)national BC guidelines recommend NAC over AC for patients with locally advanced BC (stage III) aged $<70$ years, while NAC can also be considered for patients with stage II BC with a clear indication for adjuvant chemotherapy $[1,9,10]$. The use of NAC for early BC is increasing, but despite its advantages, NAC is still applied less frequently than AC [11]. In the Netherlands, $12 \%$ of all newly diagnosed BC patients was treated with NAC in 2014, whereas in that same year $31 \%$ of patients received AC. Also, a considerable variation (0-97\%) in NAC-application between hospitals was observed [12]. Significant predictors for the use of NAC (stage III) appeared to be young age, a diagnostic MRI, large tumour size, advanced nodal disease and a negative hormone receptor status. However, not all variation could be explained by tumour and patient characteristics [13], implicating that other factors play a part in the timing of chemotherapy. Nowadays, treatment decisions are shared between the physician and patient. Important in the process of shared decision-making (SDM) is that both patient and physician are aware of a decision being required, knowing and understanding all available information on treatment options, and sharing the decision by incorporating both the physicians advice as the patient's preferences [14]. Therefore, the goal of this study was to gain insight in patients' experiences with decisions on the timing of chemotherapy for stage II and III BC.

\section{Materials and Methods}

\section{Study population}

Fifty-two hospitals were invited to participate; nineteen were willing to cooperate. We attempted an equal distribution in hospital volume (low, middle, high) and type (general, teaching, academic), and an equal geographical scatter. Patients of these hospitals were selected from the Netherlands Cancer Registry (NCR): a nationwide registry in which all newly diagnosed cancer patients are registered, hosted by the Netherlands Comprehensive Cancer Organisation (IKNL), which includes all items for the NABON Breast Cancer Audit [12]. We selected surgically treated patients (aged 18 or older) who were diagnosed with primary invasive BC stage II/III between 2013-2014 and received NAC or AC. Patients with previous malignancies and/or metastases were excluded. A sub-set of 40-50 patients per participating hospital was randomly selected, with an average of 43 per hospital. 
A total of 805 patients (367 NAC-treated, 438 AC-treated) were invited by a letter through their treating physician between August $24^{\text {th }} 2015$ and January $1^{\text {st }} 2016$ to participate in our online questionnaire. The survey was offered within a secured web-based environment named Profile [15]; paper questionnaires were provided on request. Completed questionnaires were collected until the $28^{\text {st }}$ of February 2016. Respondents gave consent on an adjective (online) form for processing their answers and merging them to their clinical data available in the NCR. Approval from the Committee of Privacy of the NCR and the Medical Ethical Committee of the Netherlands Cancer Institute - Antoni van Leeuwenhoek were obtained for this study.

\section{Questionnaire}

The thirty-five-item questionnaire (appendix) consisted of questions on SDM, completed with questions on the patients' experience and satisfaction with chemotherapy care in general. SDM was defined as by the study of Légaré et al: both health care provider and patient recognise and acknowledge that a decision is required, while knowing and understanding all best available relevant evidence, taking into account both the patient's preferences and the provider's advice [14].

Questions (Q) 1 to 9 asked about general mental and physical health and timing and type of chemotherapy received. The following questions dealt with the conditions of SDM. To determine whether patients were informed on the possible choice between NAC and AC, patients were asked whether they received information on chemotherapy prior to surgery (Q10) and whether (Q11) and with whom (oncologist, surgeon, nurse practitioner, nurse specialised on BC, general practitioner; Q12) NAC was discussed. To assess whether information on evidence of both options was provided, patients were asked if pros and cons of both NAC and AC were discussed (Q13). To determine if patient preferences were taken into account, questions were posed on whether the patient understood on what arguments the final decision was made (Q14 to Q17, Q19). The patients experienced SDM was based on questions whether they felt they shared the decision on the timing of chemotherapy (Q18) and had enough time to make a decision (Q20). In addition, to determine the overall level of patient information we asked questions on chemotherapy treatment information in general (Q21 to Q24). To determine chemotherapy treatment experience, all questions from the Cancer Therapy Satisfaction Questionnaire (CTSQ) were included (Q25 to Q30), consisting of three domains: Expectation of Therapy (EOT), Feelings about Side Effects (FSE), and Satisfaction With Therapy (SWT) [16]. General items such as nationality, level of education, and living and working status were requested as well (Q31 to Q35). A patient panel contacted through the Dutch BC patient association (Borstkankervereniging Nederland) critically reviewed and adjusted the questionnaire in comprehensible language and added additional explanations.

\section{Analysis}

Completed questionnaires were merged with the clinical data registered in the NCR.

Generalisability of the results was determined by comparing characteristics of respondents to non-respondents (Pearson's chi-square). Furthermore, NAC-treated and AC-treated respondents were compared on patient, tumour, and treatment characteristics (Pearson's chi-square). 
The answers to the questionnaire were assessed separately for stage II and III; NAC-treated compared to AC-treated patients. Conditions of SDM were chi-square tested, as well as the experience with general information on chemotherapy (Q21 to Q24). At last, treatment experience was described by calculating CTSQ-scores [17]: a score between 0 and 100 was assessed separately for each domain for respondents that answered a minimum amount of questions. Higher score $s$ are associated with better responses (better therapy expectations, feeling less impact of side effects, and greater satisfaction with therapy). Means were calculated by the sum of all assessed scores divided by the number of respondents that a score was assessed to. Mean scores were compared using a T-test; we reported $95 \%$-confidence intervals as well. Statistical significance was defined as a $p$-value $<0.05$ (two-sided).

All analyses were performed in STATA 14.

\section{Results}

\section{Respondents to questionnaire (Table 1)}

A response rate of 49\% (394/805) was reached; 179 (45\%) NAC-treated patients versus 215 (55\%) AC-treated patients. Respondents did not differ significantly from the non-respondents on patient (age), tumour (year of diagnosis, clinical stage, morphology), and hospital characteristics (volume, type). The ratio of NAC versus AC was comparable between respondents and non-respondents.

NAC-treated respondents were more often treated in a teaching or academic hospital (including BC-specialised hospitals) and in a high-volume hospital. Moreover, they were generally younger and had a higher SES, and were more often classified with clinical stage III disease (30\%) compared to AC-treated patients (7\%). Also, there were more triple-negative and Her2-receptor positive tumours in the NAC-treated group. The majority of NAC-treated patients received breast conserving surgery for BC stage II (58\%); AC-treated patients received a mastectomy more often $(54 \%$, all $p<0.05)$.

Table 1: characteristics respondents treated with NAC vs AC

\begin{tabular}{|c|c|c|c|c|c|}
\hline & $\operatorname{NAC}(n=179)$ & (\%) & $A C(n=215)$ & (\%) & $\mathrm{P}$ (Chi2) \\
\hline \multicolumn{6}{|c|}{ Patient characteristics } \\
\hline \multicolumn{6}{|c|}{ Age at diagnosis } \\
\hline$<40$ & 19 & $11 \%$ & 14 & $7 \%$ & \\
\hline $40-49$ & 74 & $41 \%$ & 65 & $30 \%$ & $<0.001$ \\
\hline $50-59$ & 55 & $31 \%$ & 58 & $27 \%$ & \\
\hline $60+$ & 31 & $17 \%$ & 78 & $36 \%$ & \\
\hline \multicolumn{6}{|l|}{ Comorbidities } \\
\hline None & 119 & $66 \%$ & 140 & $65 \%$ & \\
\hline 1 & 48 & $27 \%$ & 59 & $27 \%$ & 0.987 \\
\hline 2 or more & 10 & $6 \%$ & 13 & $6 \%$ & \\
\hline Missing & 2 & $1 \%$ & 3 & $1 \%$ & \\
\hline
\end{tabular}

Table 1 continues on next page 


\begin{tabular}{|c|c|c|c|c|c|}
\hline \multicolumn{6}{|l|}{ Socio-economic status (SES)* } \\
\hline High & 65 & $36 \%$ & 55 & $26 \%$ & \multirow{3}{*}{0.008} \\
\hline Medium & 73 & $41 \%$ & 82 & $38 \%$ & \\
\hline Low & 41 & $23 \%$ & 78 & $36 \%$ & \\
\hline \multicolumn{6}{|l|}{ Highest completed level of education } \\
\hline Secondary school (low level) or lower & 19 & $11 \%$ & 43 & $20 \%$ & \multirow{6}{*}{0.093} \\
\hline Secondary school (medium level) & 38 & $21 \%$ & 49 & $23 \%$ & \\
\hline Secondary school (high level) & 22 & $12 \%$ & 26 & $12 \%$ & \\
\hline Intermediate vocational training (MBO) & 39 & $22 \%$ & 41 & $19 \%$ & \\
\hline $\begin{array}{l}\text { Higher vocational training (HBO) and } \\
\text { university }\end{array}$ & 58 & $32 \%$ & 50 & $23 \%$ & \\
\hline Other/unknown & 3 & $2 \%$ & 6 & $3 \%$ & \\
\hline \multicolumn{6}{|c|}{ Tumour characteristics } \\
\hline \multicolumn{6}{|l|}{ Stage (short), clinical } \\
\hline ॥ & 126 & $70 \%$ & 201 & $93 \%$ & \multirow[t]{2}{*}{$<0.001$} \\
\hline III & 53 & $30 \%$ & 14 & $7 \%$ & \\
\hline \multicolumn{6}{|l|}{$\begin{array}{l}\text { Hormone receptor status (based on biopsy } \\
\text { supplemented with post-OK information) }\end{array}$} \\
\hline Triple negative & 33 & $18 \%$ & 29 & $13 \%$ & \multirow{5}{*}{0.028} \\
\hline Hormone-negative, Her2-positive & 15 & $8 \%$ & 14 & $7 \%$ & \\
\hline Hormone-positive, Her2-positive & 29 & $16 \%$ & 23 & $11 \%$ & \\
\hline Hormone-positive, Her2-negative & 99 & $55 \%$ & 149 & $69 \%$ & \\
\hline Unknown & 3 & $2 \%$ & 0 & $0 \%$ & \\
\hline \multicolumn{6}{|c|}{ Treatment characteristics } \\
\hline \multicolumn{6}{|l|}{ Type of surgery (based on final surgery) } \\
\hline \multicolumn{6}{|l|}{ Stage II (clinical) } \\
\hline Breast Conserving/Lumpectomy & 73 & $58 \%$ & 92 & $46 \%$ & \multirow[t]{2}{*}{0.032} \\
\hline Mastectomy & 53 & $42 \%$ & 109 & $54 \%$ & \\
\hline \multicolumn{6}{|l|}{ Stage III (clinical) } \\
\hline Breast Conserving/Lumpectomy & 14 & $26 \%$ & 1 & $7 \%$ & \multirow[t]{2}{*}{0.124} \\
\hline Mastectomy & 39 & $74 \%$ & 13 & $93 \%$ & \\
\hline \multicolumn{6}{|c|}{ Hospital characteristics } \\
\hline \multicolumn{6}{|l|}{ Hospital type } \\
\hline General & 47 & $26 \%$ & 79 & $37 \%$ & \multirow{2}{*}{0.026} \\
\hline $\begin{array}{l}\text { Teaching or academic (incl. BC specialised } \\
\text { hospital) }\end{array}$ & 132 & $74 \%$ & 136 & $63 \%$ & \\
\hline \multicolumn{6}{|l|}{ Hospital surgical volume $* *$} \\
\hline Low & 58 & $32 \%$ & 112 & $52 \%$ & \multirow{3}{*}{$<0.001$} \\
\hline Middle & 77 & $43 \%$ & 75 & $35 \%$ & \\
\hline High & 44 & $25 \%$ & 28 & $13 \%$ & \\
\hline
\end{tabular}

* Socio-economic status (SES) of the patients was based on four-digit postal code at time of surgery; SES scores are provided by the Netherlands Institute for Social Research (Sociaal Cultureel Planbureau) and divided into three groups based on the delivered rank numbers: low (1st-3rd deciles), intermediate (4th-7th) and high (8th-10th) SES.

** Hospitals were categorised by surgical volume for primary breast cancer, defined as the mean annual number of $B C$ surgeries during the period 2011-2015; categorised as low (<150), medium (150-300), and high (300<) volume. 


\section{Conditions of SDM (Table 2)}

For BC stage II, $98 \%$ and $84 \%$ of NAC-treated and AC-treated patients, respectively, received information on chemotherapy prior to surgery $(p=0.000)$. Among AC-treated patients, receiving information was more common in younger patients $(p=0.061)$. Further on, information was provided four times as often by the medical oncologist for NAC-treated compared to AC-treated patients respectively (Figure 1). If information on chemotherapy was provided prior to surgery, $100 \%$ of NAC-treated patients versus $14 \%$ of AC-treated patients received information on NAC as a possible treatment option ( $p=0.000)$; again, receiving information in the $A C$-group was more common in younger patients $(p=0.009)$. Of all respondents that received information on NAC, $85 \%$ and $63 \%$ of NAC and AC-patients, respectively, stated they received sufficient evidence on the pros and cons of both NAC and AC ( $p=0.008)$. Eventually, NAC-patients could explain more often why she and/or her physician decided the given treatment plan ( $97 \%$ NAC vs $66 \% A C, p=0.000$ ).

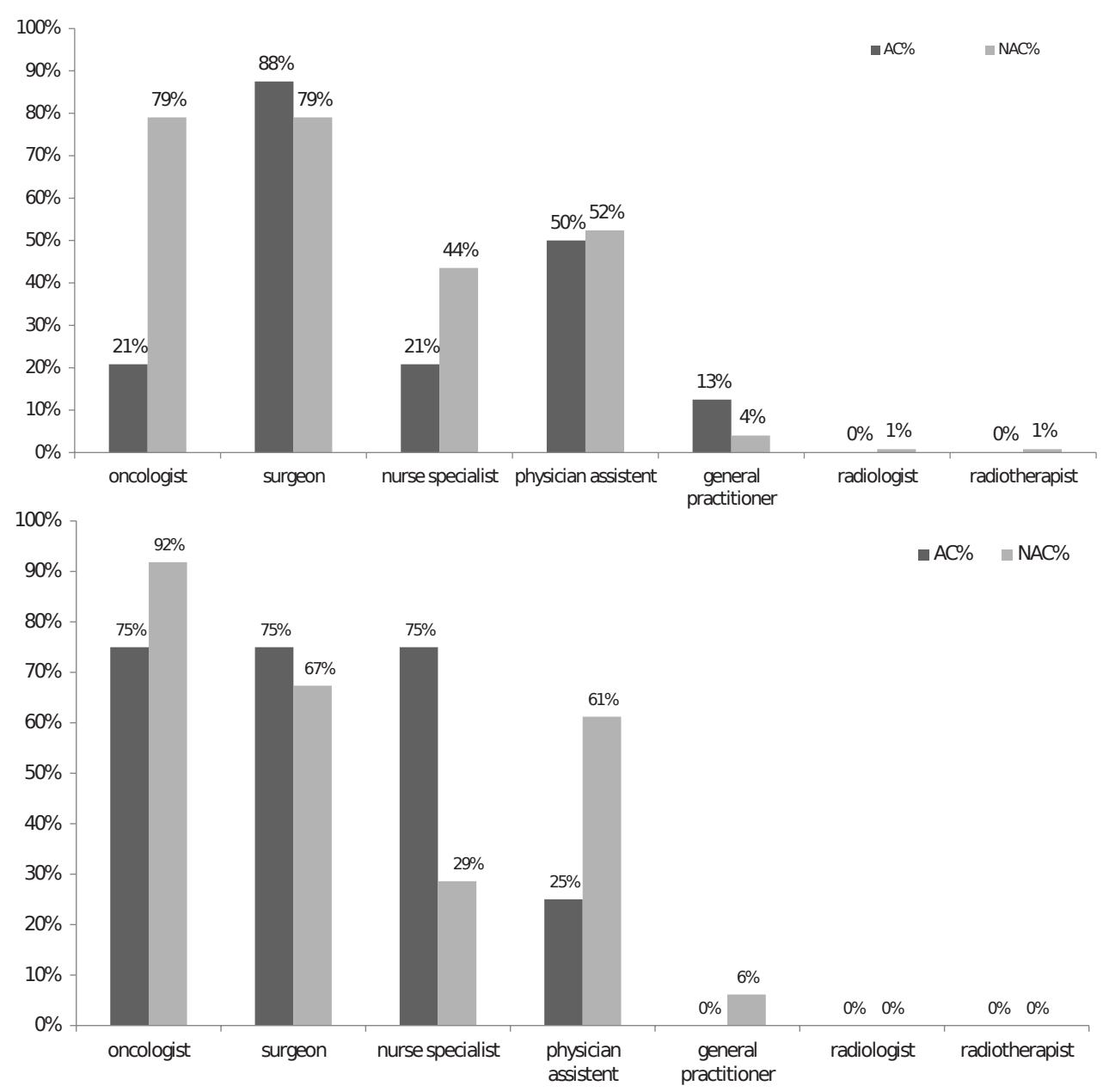

Figure 1: information on chemotherapy provided prior to surgery by physicians during pre-surgical consultation(s), NAC vs AC, stage II (a) and stage III (b) separately (Q12) 
For BC stage III, 92\% and 93\% of NAC and AC-treated patients, respectively, received prior to surgery any information on chemotherapy $(p=0.959)$. Provided information on pros and cons of NAC was stated sufficient in both groups $(p=0.947)$; almost every patient was able to explain why she and or her physician decided on either NAC or AC $(p=0.362)$ (Table 2$)$.

Table 2 : conditions of Shared Decision-Making (SDM), NAC vs AC; separate for stage II and III

\begin{tabular}{|c|c|c|c|c|c|}
\hline & NAC & (\%) & $A C$ & $(\%)$ & $\mathrm{P}($ Chi2) \\
\hline \multicolumn{6}{|l|}{ Stage II (n=126 NAC, 201 AC) } \\
\hline $\begin{array}{l}\text { Patients received information on chemotherapy in general before } \\
\text { surgery } \\
\text { ( } n=126 \text { NAC, } 201 \mathrm{AC})\end{array}$ & 124 & $98 \%$ & 169 & $84 \%$ & $<0.001$ \\
\hline $\begin{array}{l}\text { Patient was given information about NAC } \\
(n=124 N A C, 169 A C)\end{array}$ & 124 & $100 \%$ & 24 & $14 \%$ & $<0.001$ \\
\hline $\begin{array}{l}\text { Patient received sufficient information on pros and cons of both } \\
A C \text { and NAC } \\
(n=124 N A C, 24 A C)\end{array}$ & 106 & $85 \%$ & 15 & $63 \%$ & 0.008 \\
\hline $\begin{array}{l}\text { Patient was able to explain why she and/or the physician chose } \\
\text { for either NAC or AC } \\
\text { ( } n=126 \text { NAC, } 201 \text { AC) }\end{array}$ & 122 & $97 \%$ & 132 & $66 \%$ & $<0.001$ \\
\hline \multicolumn{6}{|l|}{ Stage III ( $n=53$ NAC, 14 AC) } \\
\hline $\begin{array}{l}\text { Patients received information on chemotherapy in general before } \\
\text { surgery } \\
\text { ( } n=53 \mathrm{NAC}, 14 \mathrm{AC} \text { ) }\end{array}$ & 49 & $92 \%$ & 13 & $93 \%$ & 0.959 \\
\hline $\begin{array}{l}\text { Patient was given information about NAC } \\
(n=49 N A C, 13 \mathrm{AC})\end{array}$ & 49 & $100 \%$ & 4 & $31 \%$ & $<0.001$ \\
\hline $\begin{array}{l}\text { Patient received sufficient information on pros and cons of both } \\
A C \text { and NAC } \\
(n=49 N A C, 4 A C)\end{array}$ & 36 & $73 \%$ & 3 & $75 \%$ & 0.947 \\
\hline $\begin{array}{l}\text { Patient was able to explain why she and/or the physician chose } \\
\text { for either NAC or AC } \\
\text { ( } n=53 \text { NAC, 14AC) }\end{array}$ & 50 & $94 \%$ & 14 & $100 \%$ & 0.362 \\
\hline
\end{tabular}

\section{The patient's opinion on SDM (Table 3, Figure 2)}

About half of all respondents with stage II BC (54\% NAC, 36\% AC) felt they had a real choice in their treatment plan $(p=0.004) ; 68 \%$ and $50 \%$ of NAC-treated and AC-treated patients, respectively, described they wanted to decide themselves or shared their decision with their physician (Figure 2). However, patients who stated they received information on the possibility of chemotherapy (Q10) and NAC specifically (Q11) in both groups felt equally involved in making a decision (54\% NAC, $58 \%$ AC, $p=0.854$ (not in Table)). For BC stage III, the treatment plan was already decided in $64 \%$ of NAC-treated patients and $50 \%$ of AC-treated patients $(p=0.521)$ (Table 3$)$. 
Table 3: The patients opinion on Shared Decision-Making (SDM), NAC vs AC; separate for stage II and III

\begin{tabular}{|c|c|c|c|c|c|}
\hline & $\begin{array}{c}\text { NAC } \\
(n=126)\end{array}$ & (\%) & $\begin{array}{c}A C \\
(n=201)\end{array}$ & $(\%)$ & $P($ Chi2) \\
\hline \multicolumn{6}{|l|}{ Stage II (n=126 AC, $201 \mathrm{AC})$} \\
\hline $\begin{array}{l}\text { Patient felt she did have a choice in either choosing for NAC } \\
\text { or AC } \\
\text { ( } n=126 \text { NAC, } 201 \text { AC) }\end{array}$ & 68 & $54 \%$ & 72 & $36 \%$ & 0.008 \\
\hline $\begin{array}{l}\text { Patient felt she had enough time to decide on either NAC or } \\
\text { AC } \\
(n=68 N A C, 72 A C)\end{array}$ & 67 & $99 \%$ & 72 & $100 \%$ & 0.302 \\
\hline \multicolumn{6}{|l|}{ Stage III ( $n=53$ NAC, 14 AC) } \\
\hline $\begin{array}{l}\text { Patient felt she did have a choice in either choosing for NAC } \\
\text { or } A C \\
(n=53 \text { NAC, 14AC) }\end{array}$ & 19 & $36 \%$ & 7 & $50 \%$ & 0.521 \\
\hline $\begin{array}{l}\text { Patient felt she had enough time to decide on either NAC or } \\
\text { AC } \\
(n=19 N A C, 7 A C)\end{array}$ & 19 & $100 \%$ & 7 & $100 \%$ & 0.923 \\
\hline
\end{tabular}
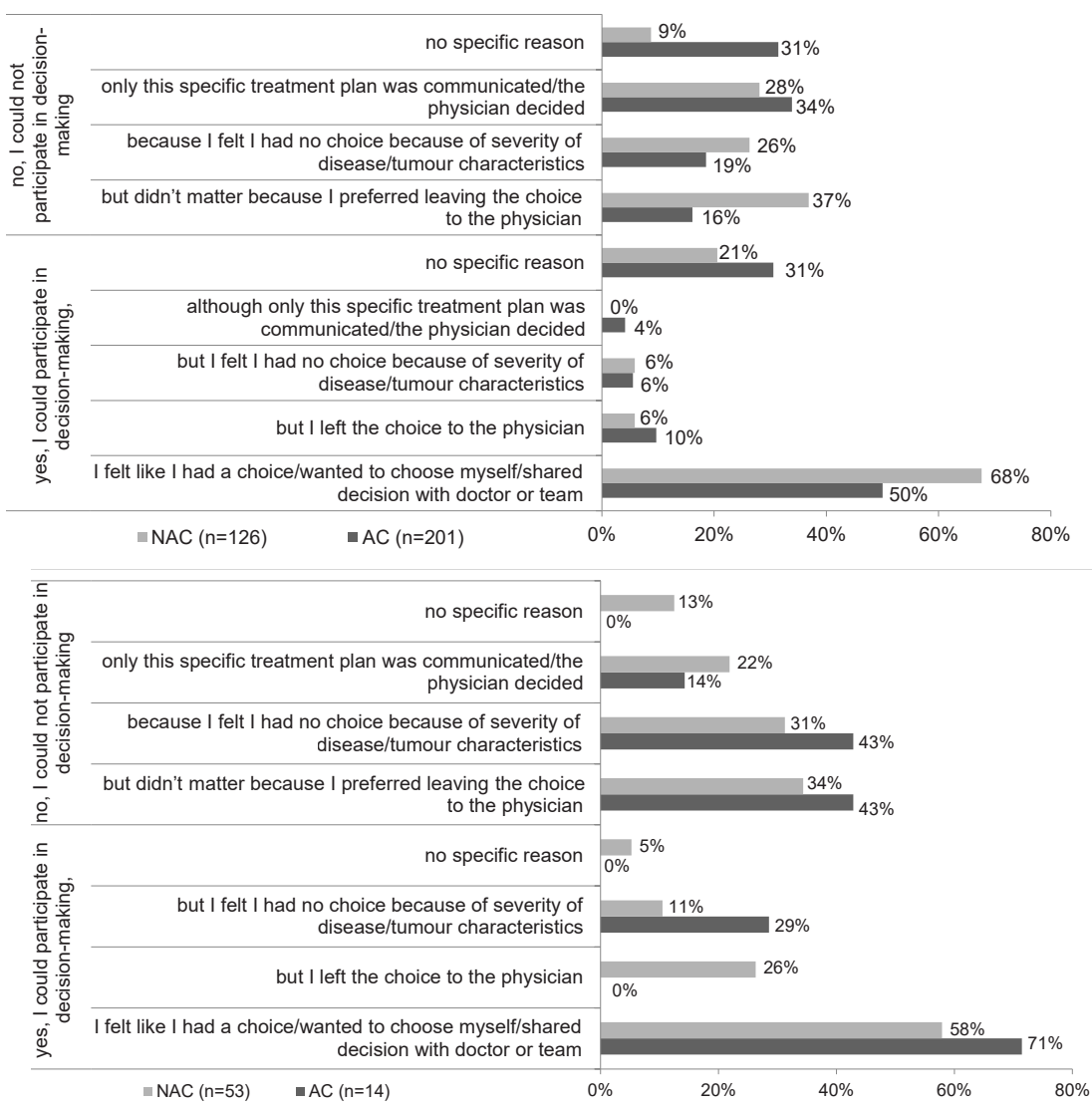

Figure 2: The patients opinion on SDM, separate for stage II (a) and III (b) (Q18, categorization of free text fields) 


\section{Experience with general information on chemotherapy}

No significant differences were found in the patients' experience with general information on chemotherapy. Over $95 \%$ of all respondents received information on their chemotherapy scheme and understood this information ( $95 \%$ NAC, $96 \%$ AC). Over $80 \%$ of respondents was informed on side-effects of their chemotherapy (NAC $88 \%$, AC 84\%). Both groups scored very high regarding understanding the information they received on chemotherapy ( $94 \%$ NAC, 96\% AC). Respondents felt they had the opportunity to ask questions about chemotherapy ( $92 \% \mathrm{NAC}, 95 \% \mathrm{AC}$ ).

\section{Treatment experience with chemotherapy (CTSQ)}

Significant differences between NAC-treated and AC-treated patients, respectively, were found in the treatment experience (Figure 3). Mean EOT-scores for both NAC-treated and AC-treated patients were high (68 NAC, 68 AC; $p=0.948$ ), meaning that overall, respondents had a high believe in chemotherapy contributing to their cancer treatment. FSE-scores were moderate (46 NAC, 45 $A C ; p=0.714)$, meaning respondents felt their side effects were as severe as expected beforehand. In totality, NAC-treated patients were less satisfied with their chemotherapy than AC-treated patients (40 NAC, $42 \mathrm{AC} ; \mathrm{p}=0.018$ ).

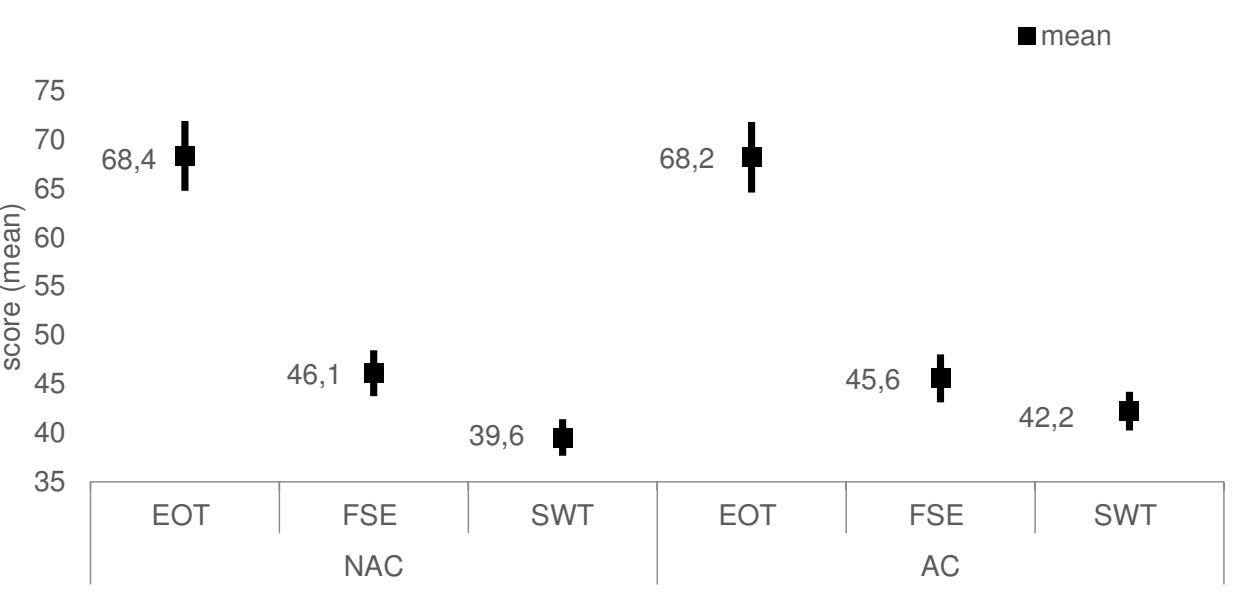

Figure 3: treatment experience, mean CTSQ-scores per domain* and chemotherapy treatment, incl. 95\% lower and upper confidence interval for NAC and AC seperately ( $n=179$ NAC, 215 AC)

*domains: Expectation of Therapy (EOT), Feelings about Side Effects (FSE), and Satisfaction With Therapy (SWT); range 0 (low score) to 100 (high score);

\section{Discussion}

This study highlights important aspects in the decision-making process on the timing of chemotherapy (NAC vs AC) for early breast cancer. If information on chemotherapy was provided prior to surgery, $100 \%$ of NAC-treated patients versus $14 \%$ of AC-treated patients received information on NAC as a possible treatment option. Of those who received information on NAC, $85 \%$ and $63 \%$ of patients treated with NAC and AC, respectively, stated that they received sufficient evidence on the pros and cons of both NAC and AC. 
The results of this survey confirm that the choice regarding either NAC or AC is often not discussed with patients with stage II-III breast cancer prior to treatment. This suggests that clinicians rarely express that a treatment decision needs to be made, and patients may not realize that neoadjuvant treatment is a valid choice. In order to make a decision, sufficient information and relevant evidence on pros and cons of all treatment options should be provided before the start of therapy. Patients treated with AC were less informed about this treatment decision than NAC-treated respondents, and stage II respondents were less informed than stage III respondents.

Further on, both patients' and clinicians' preferences should be incorporated in treatment plans [14]. Few AC-treated respondents with BC stage II were able to explain reasons for adjuvant timing of systemic treatment instead of neoadjuvant timing. Moreover, about half of respondents did not feel they had a choice regarding timing of systemic treatment. These results reveal the impaired role of participation of patients in SDM on NAC.

Several potential explanations are present. First, the Dutch and international breast guidelines are straightforward about the recommendation of NAC for stage III BC $[1,9,10]$, but the evidence of NAC for stage II BC is not included in the guidelines yet, since it is based on promising preliminary data and research $[18,19]$. Seemingly, treatment decisions are predominantly guideline-congruent, and when guidelines are not clear, clinicians' recommendations to patients are not uniform either. Consequently, differences in clinicians' opinions may lead to variation in treatment patterns, as confirmed by the NBCA audit results and other recent studies [11, 13].

Moreover, clinicians' opinions exert one of the most powerful influences over patients' preferences [20]. Also, patients are often not aware that a treatment decision is required [20]. The health professional first speaking with the patient plays an important role in how information is conveyed, whether this is a surgeon, medical oncologist, nurse practitioner, or physician assistant. This will most likely drive the treatment decision. According to our survey, most of the information about NAC was provided by medical oncologists, of whom we expect stronger support for applying NAC than from surgeons. A referral from the surgeon to the medical oncologist defines whether a patient actually will have a consultation with an oncologist. In addition, appropriate information and additional support is essential to make quality decisions. Decision support-systems may help patients allow them to be primary decision maker [21]. Thirdly, the level of training of clinicians, conference attendance, and multidisciplinary meeting groups on a regular base may play a crucial role by creating more choice-awareness in preference-sensitive decisions.

Also, the preferred role of patients in preference-sensitive decisions is influenced by patients' age and education. Older and less educated patients are more likely to prefer a passive or collaborative role [22], whereas an active role is generally preferred by younger and better educated patients [23]. We observed that NAC-treated patients treated were younger and better educated, while being better informed as well. Patient's participation in their treatment plan is important because a high level of involvement is associated with improved outcome in quality of life, physical and social functioning. Patient's involvement led to high levels of satisfaction with the decision and the subsequent treatment they received [24]. Even if the fit of treatment to preferences is not 
enhanced, the fact that patients are involved and felt meaningful to the situation may increase satisfaction [25]. Moreover, patients could be pleased to know whether their tumour responded or progressed on NAC, which can be valuable contribution under conditions of uncertainty.

Finally, the most common explanations for the application of NAC in stage II and stage III patients were tumour size and axillary metastases. These explanations correspond with reported results from cancer registries $[11,13]$. While NAC aims to downsize the tumour to improve the possibility of breast conservation surgery, it was expected that more respondents were treated with BCS after NAC $[4,26]$. However, in our survey the patient's desire for BCS was the major reason for NAC in only $28 \%$ of BC stage II patients. Valid options to refrain from chemotherapy (NAC or postoperative) may have been contraindications such as poor performance status or severe comorbidity. Also, it may be possible that women prefer to undergo surgery first, but these considerations should be clearly discussed.

Overall, the results of our study showed that general experience (CTSQ) with chemotherapy in terms of side effects was scored equal in both groups, but significant differences between groups were found in final satisfaction with care; NAC-treated patients seem to be less satisfied. The most likely explanation for this result, is the difference in approach in NAC-treated patients, in which NAC is commonly being used for down- staging of the tumour to increase resectability and enable BCS. When the response to chemotherapy appears to be disappointing and BCS does not seem possible, satisfaction could be less. Also, mostly young women receive NAC, which will have a big impact on their daily lives. However, these negative perceptions reiterate the importance of counselling support, communication, and expectation management.

\section{Strengths and limitations}

To our knowledge, this is the first study reflecting patients' experiences with decisions on the timing of chemotherapy for early BC. Previous literature focused mainly on experiences with decisions on adjuvant therapies for $B C[27,28]$. In the context of an increasing trend in NAC use[11, 26], insight in patients' experience in SDM on NAC is extremely relevant.

Because of the connection between the clinical cancer registry and the patient reported experiences, this study is unique in design. We reached a high response rate of almost 400 respondents (49\%) and selected a homogeneous comparable population compared to non-responders. The absolute number of BC stage III respondents was small, but because of the strict indication of NAC for these patients, this group was less relevant to discuss. Participation was opt-in, leading to selection bias in which those who responded were probably more critical on SDM then non-responders; providing paper questionnaires on request could lead to an underrepresentation of patients with lack of computer skills or access. Also, recall bias is a known limitation of all survey studies. Idem, creating a patient-comprehensible questionnaire is a difficult task. We were favoured by input from the Dutch patient association on breast cancer. Besides, we emphasize the fact that patient-reported data is based on the experience of patients, rather than a factual reflexion of how decisions on chemotherapy timing were made. Furthermore, unfortunately, the numbers of 
respondents per hospital were too small to analyse intra-hospital variation in SDM; this would be interesting for further research.

\section{Conclusion}

In conclusion, our study revealed that the need to make a treatment decision on the timing of chemotherapy (NAC vs AC) for early breast cancer was discussed with only a small number of patients, in particular in BC stage II. Less than half of the respondents felt they had a real choice. Clinicians' opinions exert one of the most powerful influences over patients' preferences. National guidelines that are frequently updated and a continuous audit system integrating detection and real-time feedback will help in providing clear guidance to physicians for chemotherapy treatment timing with decision-making as a result of team work of all involved disciplines. By understanding patient preferences and incorporating them into treatment decisions, it will be possible to reduce unwarranted variations and deliver appropriate patient-centred care. 


\section{References}

1. Netherlands Comprehensive Cancer Organisation (IKNL). National guideline on Breast Cancer. Netherlands Comprehensive Cancer Organisation (IKNL); 2012.

2. Early Breast Cancer Trialists' Collaborative Group (EBCTCG). Effects of chemotherapy and hormonal therapy for early breast cancer on recurrence and 15-year survival: an overview of the randomised trials. Lancet. 2005;365(9472):1687-717.

3. Mauri D, Pavlidis N, loannidis JP. Neoadjuvant versus adjuvant systemic treatment in breast cancer: a metaanalysis. J Natl Cancer Inst. 2005;97(3):188-94.

4. Mieog JS, van der Hage JA, van de Velde CJ. Preoperative chemotherapy for women with operable breast cancer. Cochrane Database Systematic Review. 2007;CD005002.

5. Rastogi P, Anderson SJ, Bear HD, Geyer CE KM, Robidoux A, Margolese RG, et al. Preoperative chemotherapy: updates of National Surgical Adjuvant Breast and Bowel Project Protocols B-18 and B-27. J Clin Oncol. 2008;26(5):778-85.

6. Mougalian SS, Hernandez M, Lei X, Lynch S, Kuerer HM, Symmans WF, et al. Ten-Year Outcomes of Patients With Breast Cancer With Cytologically Confirmed Axillary Lymph Node Metastases and Pathologic Complete Response After Primary Systemic Chemotherapy. JAMA Oncology. 2016;2(4):508-16.

7. Symmans WF, Wei C, Gould R, Yu X, Zhang Y, Liu M, et al. Long-Term Prognostic Risk After Neoadjuvant Chemotherapy Associated With Residual Cancer Burden and Breast Cancer Subtype. Journal of Clinical Oncology. 2017;35(10):1049-60.

8. Untch M, Jackisch C, Schneeweiss A, Conrad B, Aktas B, Denkert C, et al. Nab-paclitaxel versus solvent-based paclitaxel in neoadjuvant chemotherapy for early breast cancer (GeparSepto-GBG 69): a randomised, phase 3 trial. Lancet Oncol. 2016;17:345-56.

9. Holmes D, Colfry A, Czerniecki B, Dickson-Witmer D, Francisco Espinel C, Feldman E, et al. Performance and Practice Guideline for the Use of Neoadjuvant Systemic Therapy in the Management of Breast Cancer. Ann Surg Oncol. 2015;22(10):3184-90.

10. Cardoso F, Costa A, Norton L, Senkus E, Aapro M, André F, et al. ESO-ESMO 2nd international consensus guidelines for advanced breast cancer (ABC2). The Breast. 2014;23(5):489-502.

11. Mougalian SS, Soulos PR, Killelea BK, Lannin DR, Abu-Khalaf MM, DiGiovanna MP, et al. Use of neoadjuvant chemotherapy for patients with stage I to III breast cancer in the United States. Cancer. 2015;121(15):254452.

12. Dutch Institute for Clinical Auditing. Jaarrapportage 2013. NABON Breast Cancer Audit; 2014.

13. Spronk PER, van Bommel ACM, Vrancken Peeters MTFD, Siesling S, Smorenburg CH. Variation in the use of neoadjuvant chemotherapy for stage III breast cancer: results of the Dutch nationwide breast cancer registry NBCA (Nabon Breast Cancer Audit). The Breast. 2017;36:34-8.

14. Légaré F, Witteman HO. Shared Decision Making: Examining Key Elements And Barriers To Adoption Into Routine Clinical Practice. Health Affairs. 2013;32(2):276-84.

15. Netherlands Comprehensive Cancer Organisation, University of Tilburg. PROFILES registry [Available from: https://www.profilesregistry.nl/Home.

16. Trask PC, Tellefsen C, Espindle D, Getter C, Hsu MA. Psychometric Validation of the Cancer Therapy Satisfaction Questionnaire. Value in Health. 2008;11(4):669-79.

17. Administration and Scoring Guide for the Cancer Therapy Satisfaction Questionnaire (CTSQ). 2006 31-102006.

18. von Minckwitz G, Untch M, Nüesch E, Loibl S, Kaufmann M, Kümmel S, et al. Impact of treatment characteristics on response of different breast cancer phenotypes: pooled analysis of the German neoadjuvant chemotherapy trials. Breast Cancer Res Treat. 2011;125(1):145-56.

19. Cortazar P, Zhang L, Untch M, Mehta K, Costantino JP, Wolmark N, et al. Pathological complete response and long-term clinical benefit in breast cancer: the CTNeoBC pooled analysis. Lancet. 2014;384(9938):164-72.

20. Kunneman M, Engelhardt EG, Ten Hove FL, Marijnen CA, Portielje JE, Smets EM, et al. Deciding about (neo-) adjuvant rectal and breast cancer treatment: Missed opportunities for shared decision making. Acta Oncol. 2016;55(2):134-9. 
21. Zdenkowski N, Butow P, Hutchings E, Douglas C, Coll JR, Boyle FM. A Decision Aid for Women Considering Neoadjuvant Systemic Therapy for Operable Invasive Breast Cancer: Development and Protocol of a Phase II Evaluation Study (ANZ1301 DOMINO). JMIR Res Protoc. 2016;5(2).

22. Mandelblatt J, Kreling B, Figeuriedo M, Feng S. What is the impact of shared decision making on treatment and outcomes for older women with breast cancer? J Clin Oncol. 2006;24(30):4908-13.

23. Wallberg B, Michelson H, Nystedt M, Bolund C, Degner LF, Wilking N. Information Needs and Preferences for Participation in Treatment Decisions Among Swedish Breast Cancer Patients. Acta Oncol. 2000;39(4):467-76.

24. Hamelinck VC, Bastiaannet E, Pieterse AH, Jannink I, van de Velde CJ, Liefers GJ. Patients ' preferences for sur- gical and adjuvant systemic treatment in early breast cancer: A systematic review Cancer Treat Rev. 2014;40:1005-18.

25. Stiggelbout AM, Van der Weijden T, De Wit MP, Frosch D, Légaré F, Montori VM, et al. Shared decision making: really putting patients at the centre of healthcare. BMJ. 2012;344 :e256

26. Killelea BK, Yang VQ, Mougalian S, et al. Neoadjuvant Chemotherapy for Breast Cancer Increases the Rate of Breast Conservation: Results from the National Cancer Database. . Journal of the American College of Surgeons. 2015;220:1063-9.

27. Wengstrom Y, Aapro M, Leto di Priolo S, Cannon H, Georgiou V. Patients' knowledge and experience of adjuvant endocrine therapy for early breast cancer: a European study. Breast (Edinburgh, Scotland). 2007;16:462-8.

28. Gaston CM, Mitchell G. Information giving and decision- making in patients with advanced cancer: a cancer systematic review. Soc Sci Med. 2005;61:2252-64. 
Supplementary table 1: questionnaire (plain text)

0. What is your date of birth? ..-..-19..

1. How was, in your own perception, your physical health over the past three months?

Excellent - very well - well - moderate - bad

2. How was, in your own perception, your mental health over the past three months?

Excellent - very well - well - moderate - bad

3. At time of your treatment with chemotherapy, did you suffer from one or more of undermentioned diseases?

Any other type of cancer - lung disease - cardiovascular disease - gastro-intestinal disease - illness of urinary or reproductive system - musco-skeletal disease - central nerve system - illness of metabolism or coagulopathy - infectious disease - none - other

4. Were you menopausal at time of your treatment with chemotherapy?

Premenopausal - perimenopausal - postmenopausal - I don't know - not applicable

5. Were you treated with chemotherapy anterior or posterior to your surgical treatment for breast cancer?

Anterior (neoadjuvant chemotherapy) - posterior (adjuvant chemotherapy) - both anterior as posterior (combination of neoadjuvant and adjuvant)

6. Which type of chemotherapy did you receive at the start of your chemotherapy treatment?

TAC - AC - FEC - FEC followed by taxane - AC followed by taxane - Trastuzumab (Herceptin) and chemotherapy - I don't know - other

7. How many courses of chemotherapy treatment did you receive?

.. courses

8. Did you finish the total amount of chemotherapy courses that were planned for you?

Yes (proceed with question 10) - no (proceed with question 9)

9. What was the reason for premature termination of your chemotherapy treatment?

Because of (severe) side effects - by own preference - the chemotherapy did not (sufficient) affect the cancer - I don't know - other

10. Was the necessity of chemotherapy within your treatment plan discussed with you previous to receiving your surgical treatment?

$$
\text { Yes - no - I don't know }
$$

11. Was the option of starting of with chemotherapy prior to surgery discussed with you previous to receiving your surgical treatment?

$$
\text { Yes - no - I don't know }
$$

12. With whom did you discuss treatment with chemotherapy prior to surgery?

Oncologist - surgeon - nurse practitioner - breast cancer nurse - general practitioner - other 
13. Did you receive information on the pros and cons of both treatment with chemotherapy initiated either prior or subsequently to surgical treatment?

Yes - yes, but not as much as I preferred - no - I don't know

14. Do you know why you were treated with chemotherapy prior to receiving surgery?

Not applicable, I received adjuvant chemotherapy treatment - No, I don't know - Yes, I do know

15. In case you do know why you were treated with chemotherapy prior to surgery, what was the reason for choosing this treatment option?

Tumour size - axillary metastases - preferring a breast conserving surgery - to stretch time to surgery

- my physician decided this - my physician decided this, because... - I don't know - other

16. Do you know why you were treated with chemotherapy after receiving surgery?

Not applicable, I received neoadjuvant chemotherapy treatment - No, I don't know - Yes, I do know

17. In case you do know why you were treated with chemotherapy after receiving surgery, what was the reason for choosing this treatment option?

Tumour size - axillary metastases - I preferred this type of chemotherapy - my physician decided this - my physician decided this, because... - I don't know - other

18. Do you feel you could co-decide with your physician on treatment with chemotherapy either prior or after receiving surgery?

Yes, because... - no, because...

19. Who helped you in deciding on chemotherapy treatment order?

I decided myself - my physician - my partner - family - friends - information on the internet - patient association - 'fellow-sufferers' - other

20. Do you feel you had enough time to decide on chemotherapy treatment order?

Yes - no

21. Was the chemotherapy treatment scheme explained clearly to you?

Yes, and I fully understood the explanation - yes, but I did not fully understand the explanation - no

- I don't know

22. Were the side effects of chemotherapy explained prior to receiving chemotherapy?

Yes, prior to treatment and sufficient - Yes, prior to treatment but not sufficient - Yes, but not prior to treatment - No - I don't know

23. Did you understand the information you received on chemotherapy?

Yes - no - not applicable, I received no information on chemotherapy in general - I don't know

24. Was there the opportunity to ask questions to your physician on chemotherapy?

Yes, sufficient - yes, somewhat - no - I don't know

25. How often during chemotherapy treatment did you feel that..

(never - rarely - sometimes - mostly - always)

chemotherapy would help you to return to a normal life?

b chemotherapy would get rid of the cancer?

c chemotherapy would help prevent the cancer from coming back? 
26. Overall, how worthwhile was your chemotherapy?

27. Overall, was taking chemotherapy as difficult as expected?

A lot more difficult - slightly more difficult - as difficult as I expected - slightly easier - a lot easier

28.a Overall, how well did the benefits of chemotherapy meet your expectations?

28.b Overall, were side effects as expected?

A lot better/more than expected - slightly better/more than expected - met my expectations slightly less than expected - a lot less than expected

29.a How satisfied were you with the form of your chemotherapy?

29.b How satisfied were you with your most recent chemotherapy?

Very satisfied - satisfied - neither satisfied nor dissatisfied - dissatisfied - very dissatisfied

30.a If given choice again, would you decide to take this chemotherapy treatment?

30.b Would you recommend this type of chemotherapy to others in a similar situation?

Absolutely - probably - I don't know - probably not - absolutely not

31. At time of your breast cancer treatment, what were the four digits of your postal code?

32. What is your highest completed education? (completed with diploma or certificate)

No education - lower education - middle education - higher education - other

33. What is currently your marital status?

Married/relationship - divorced/separated - widow/widower/partner diseased - single

34. What description is most applicable to you at this moment? (please tick one answer)

Attending school/education - paid employment - unemployed/seeking work - incapacitated housewife - retirement

35. What is your nationality?

Dutch - Moroccan - Surinamese - Turkish - German - Belgian - Other

Do you have any questions/remarks? 


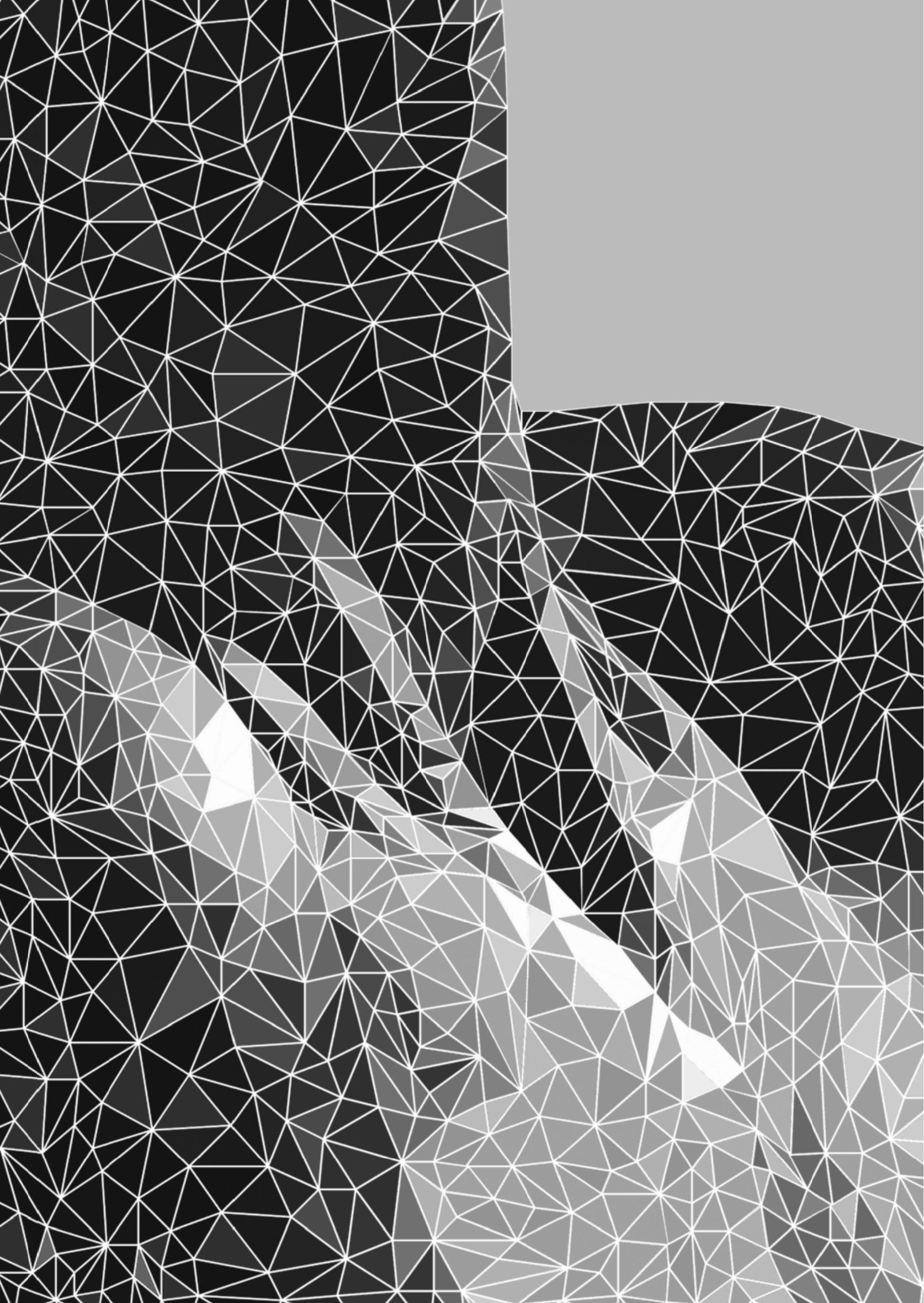


CHAPTER 2

CURRENT DECISIONS ON NEOADJUVANT CHEMOTHERAPY FOR EARLY BREAST CANCER: EXPERTS' EXPERIENCES IN THE NETHERLANDS

P.E.R. Spronk

K.M. de Ligt

A.C.M. van Bommel

S. Siesling

C.H. Smorenburg

M.T.F.D. Vrancken Peeters

- on behalf of the Nabon Breast Cancer Audit group -

Patient Educ Couns. 2018 Dec;101(12):2111-2115. 



\section{Abstract}

Purpose:

To evaluate the opinion of surgical and medical oncologists on neoadjuvant chemotherapy (NAC) for early breast cancer.

\section{Methods:}

Surgical and medical oncologists $(\mathrm{N}=292)$ participating in breast cancer care in the Netherlands were invited for a 20-question survey on the influence of patient, disease, and management related factors on their decisions towards NAC.

\section{Results:}

A total of 138 surgical and medical oncologists from 64 out of 89 different Dutch hospitals completed the survey. NAC was recommended for locally advanced breast cancer (94\%) and for downstaging to enable breast conserving surgery (BCS) (75\%). Despite willingness to downstage, $64 \%$ of clinicians routinely recommended NAC when systemic therapy was indicated preoperatively as based on known tumour characteristics. Reported reasons to refrain from NAC are comorbidities (68\%), age $>70$ years (52\%), and WHO-performance status $\geq 2$ (93\%). Opinions on NAC and surgical management were inconclusive; while $75 \%$ recommends NAC to enable BCS, some stated that BCS after NAC increases the risk of a non-radical resection (21\%), surgical complications (9\%) and recurrence of disease $(5 \%)$.

\section{Conclusion:}

This article highlights the complexity chemotherapy timing decision-making for early breast cancer patients and it emphasizes the need for more consensus among specialists on the indications for NAC in early BC patients. Unambiguous and evidence-based treatment information could improve doctor-patient communication, supporting the patient in chemotherapy timing decision-making. 


\section{Introduction}

Neoadjuvant chemotherapy (NAC) is an important initial strategy for the management of operable breast cancer $(\mathrm{BC})$. In accordance with international guidelines, the Dutch national breast cancer guideline recommends NAC as an option for all patients aged $<70$ with an indication for systemic treatment, as similar overall and disease-free survival rates were demonstrated between preoperative and postoperative application of chemotherapy [1, 2, 3, 4such as diseasefree survival, event-free survival (EFS] These guidelines disclose that NAC may be used for large tumours $(\mathrm{T} 3 ;>5 \mathrm{~cm})$ to increase resectability and the rate of breast conserving surgery and axillary preserving surgery [5initially, the only conceivable surgical option. Patients and Methods Between 2007 and 2012, 168 patients from a single center received nCT. Among these patients, we focused on the ones who received $\mathrm{nCT}$ ( $\mathrm{n}=119,[70.8 \%]$ ]. Besides, chemotherapy prior to breast surgery remains a valuable therapeutic approach for the assessment of biological anti-tumour activity and clinical efficacy of new treatments [6leading to more flexible, \"tailored\" therapies. Indeed, these advantages are tenable if one assumes that the primary tumor response serves as a surrogate marker of the efficacy of PST in terms of survival. Unfortunately, this has not yet been validated. The data that are actually available show that both clinical complete response (cCR]. Furthermore, administration of NAC creates a time frame for testing on hereditary breast cancer and planning the final type of surgery, for example reconstruction surgery.

Despite these arguments in favour of NAC, large national and international variation in the application of NAC is observed between hospitals [7,8]. Previous research based on data from the NABON Breast Cancer Audit (NBCA) revealed that most variation between hospitals consists in the treatment of BC stage IIB with a national average of $40 \%$ NAC use. For BC stage III, the national average is $80 \%$. After adjustment for patient and tumour factors associated with the use of NAC, including hospital study participation, a considerable unaccountable variation still remained between all 89 Dutch hospitals $[9,10]$.

Additional factors, such as clinician preferences and the level of shared decision-making, may play a role in the application of NAC [11]. Since it has been demonstrated that clinicians' treatment recommendations exert one of the most powerful influences over patients' preferences, the clinicians' opinion on NAC is therefore of great importance [12]. Some specialists adhere firmly to their personal treatment preferences which may lie outside evidence of best practice or safety [13]. Consequently, differences in surgeons and medical oncologists' opinions may lead to unwanted variation in treatment patterns. As options of chemotherapy timing are in equilibrium for overall and disease-free survival, but NAC also yields several advantages, it is important to gain insight in the observed variation of NAC application, as each patient indicated for NAC deserves a choice in chemotherapy timing. The aim of this study is to evaluate the current opinion of surgical and medical oncologists in the Netherlands on the use of NAC and their decisions towards NAC in early breast cancer. 


\section{Methods}

\section{Participants}

On November 11, 2015, an invitation for an online survey was sent by mail to 575 surgical and medical oncologists, invited by the network of the NABON Breast Cancer Audit (NBCA), covering all Dutch hospitals that are involved in breast cancer care. A reminder was sent to non-respondents 3 weeks later and the survey was closed on January 8th, 2016.

Demographics of participating hospitals were derived from the NBCA dataset. The surgical volume of a hospital was defined as the mean annual number of breast cancer surgeries during the period 2011-2015; divided into low-volume (<150), mid-range (150-300) and high-volume (>300) categories. Type of hospital was described as academic, teaching, and general hospitals. Academic hospitals are part of a university, and both academic and teaching hospitals provide medical training to surgical residents.

\section{Survey}

The survey was developed by a multidisciplinary taskforce, including a medical oncologist, a breast cancer surgeon, a clinical epidemiologist and medical researchers. Hereafter, the survey was pre-tested and modified based on the obtained feedback. The survey consisted of 20 questions about (contra)indications and considerations for NAC and general information about the survey participants. Part one of the survey consisted of eight questions about commonly accepted indications and contraindications of NAC on the following categories: tumour characteristics (tumour size, stage and biology), patient characteristics (age, performance status and comorbidities) and clinical disease management (genetic testing and timing of final surgery) (supplement 1 ). The 5-point Likert scale was used to allow the respondent to express how much they agree or disagree. Part two of the survey consisted of four questions about other possible considerations that could influence the use of NAC (evidence in overall and disease-free survival benefit of NAC, axillary conservation surgery, risk of complications, risk of non-radical resections), using a yes/no scale. Throughout the survey there was the ability to write and add comments in the responses. To get an idea of the level of experience per specialist, demographic data, numbers of years in specialty, numbers of patients treated, and questions on study participation were included in the survey.

\section{Statistical analysis}

Frequencies and percentages were used to display responses to individual questions. Differences between surgical and medical oncologists' responses were analysed using Pearson chi-square. Statistical significance is defined as a two-sided $p$ value $<0.05$. All analyses are performed in PASW Statistics version 24 (SPSS inc Chicago, IL, USA).

\section{Results}

A total of 292 clinicians opened the online program, of whom 138 clinicians from 64 out of 89 Dutch hospitals completed the survey, leading to a response rate of $47,3 \%$. Of 138 respondent 
clinicians, 70 surgical oncologists ( $43 \%$ female, $57 \%$ male) and 68 medical oncologists ( $59 \%$ female, $41 \%$ male) participated in the survey. The respondents had been in clinical practice for a median of 12 years (range 1-35). The number of annually treated breast cancer patients varied from 50 patients for medical oncologists (range 15-110) to 70 patients for surgical oncologists (range 30110). The majority of clinicians included more than 10 patients in neoadjuvant chemotherapy trials per year. This survey represented two-third of Dutch hospitals; 22 hospitals had only one representative and 42 hospitals were represented by 2 to 7 representatives. Medical oncologists and surgical oncologists were evenly represented according to type and volume of hospitals (Table 1).

Table 1: Respondents' and affiliated hospital demographics

\begin{tabular}{|c|c|c|c|c|}
\hline & $\begin{array}{l}\text { Surgeons } \\
(\mathrm{N}=70)\end{array}$ & $\begin{array}{l}\text { Oncologists } \\
(N=68)\end{array}$ & $\begin{array}{l}\text { Hospitals } \\
(\mathrm{N}=64)\end{array}$ & $\begin{array}{c}P \\
\text { (Chi2) }\end{array}$ \\
\hline \multicolumn{5}{|l|}{ Sex } \\
\hline Male & 40 & 28 & $\mathrm{n} / \mathrm{a}$ & 0.106 \\
\hline Female & 30 & 40 & $\mathrm{n} / \mathrm{a}$ & \\
\hline \multicolumn{5}{|c|}{ Number of years in practice } \\
\hline$<10$ & 27 & 27 & $\mathrm{n} / \mathrm{a}$ & \\
\hline $10-19$ & 32 & 27 & $\mathrm{n} / \mathrm{a}$ & 0.774 \\
\hline $20+$ & 11 & 14 & $\mathrm{n} / \mathrm{a}$ & \\
\hline \multicolumn{5}{|c|}{ Number of patients per specialist/year } \\
\hline$<50$ & 8 & 24 & $\mathrm{n} / \mathrm{a}$ & 0.001 \\
\hline $50-99$ & 23 & 25 & $\mathrm{n} / \mathrm{a}$ & \\
\hline $100+$ & 32 & 15 & $\mathrm{n} / \mathrm{a}$ & \\
\hline \multicolumn{5}{|c|}{ Number of patients per physician included in NAC studies/year } \\
\hline$<10$ & 21 & 12 & $\mathrm{n} / \mathrm{a}$ & 0.001 \\
\hline$>10$ & 39 & 52 & $\mathrm{n} / \mathrm{a}$ & \\
\hline \multicolumn{5}{|l|}{ Volume of hospital* } \\
\hline$<150$ & 27 & 29 & 31 & 0.578 \\
\hline $150-300$ & 23 & 25 & 22 & \\
\hline$>300$ & 20 & 14 & 11 & \\
\hline \multicolumn{5}{|l|}{ Type of hospital* } \\
\hline General hospital & 19 & 22 & 24 & 0.281 \\
\hline Teaching hospital & 43 & 33 & 34 & \\
\hline Academic hospital & 8 & 13 & 6 & \\
\hline
\end{tabular}

* Derived from the NBCA.

\section{Survey}

Respondents rated locally advanced breast cancer $(L A B C)$ as the most distinguished indication for NAC (94\%). The second commonly accepted indication is down staging of the tumour to enable breast conserving surgery (75\%). Of all respondents, $64 \%$ "always to frequently" recommended NAC if systemic therapy is indicated preoperatively, based on known clinical tumour characteristics (Figure 1A). Reported reasons to refrain from NAC were WHO-performance status $\geq 2(93 \%)$, 
comorbidities (68\%), and age $>70$ years (52\%) (Figure 1C and 1D). A WHO-performance score of $\geq 2$, which implies an inability to carry out any work activities, was reported as the most common contraindication. Age by itself was no contraindication according to $48 \%$ of respondents. But if so, patients aged $<70$ seemed to be the main reason for restrained application of NAC. Clinical management factors, such as the time necessary for testing on hereditary breast cancer or to plan the final type of reconstructive surgery, were less frequently denominated as indications for NAC (Figure 1B).

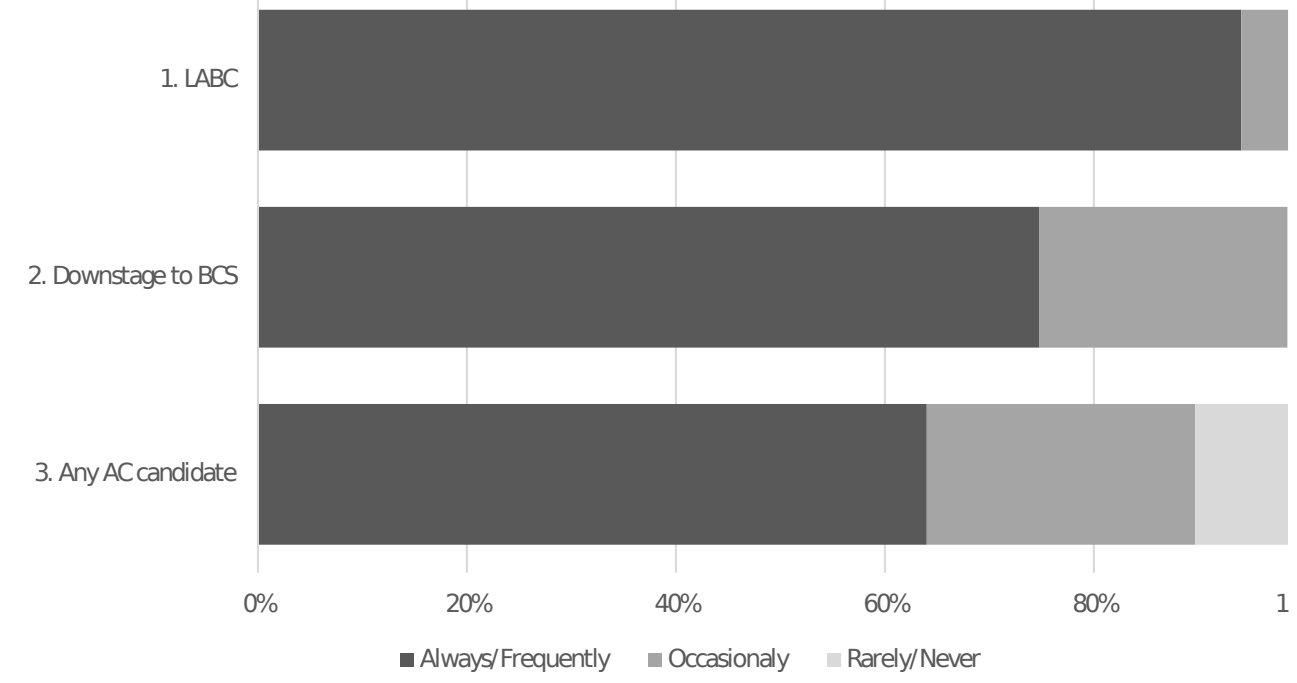

Figure 1A: Reported indications (tumour characteristics) for recommending NAC

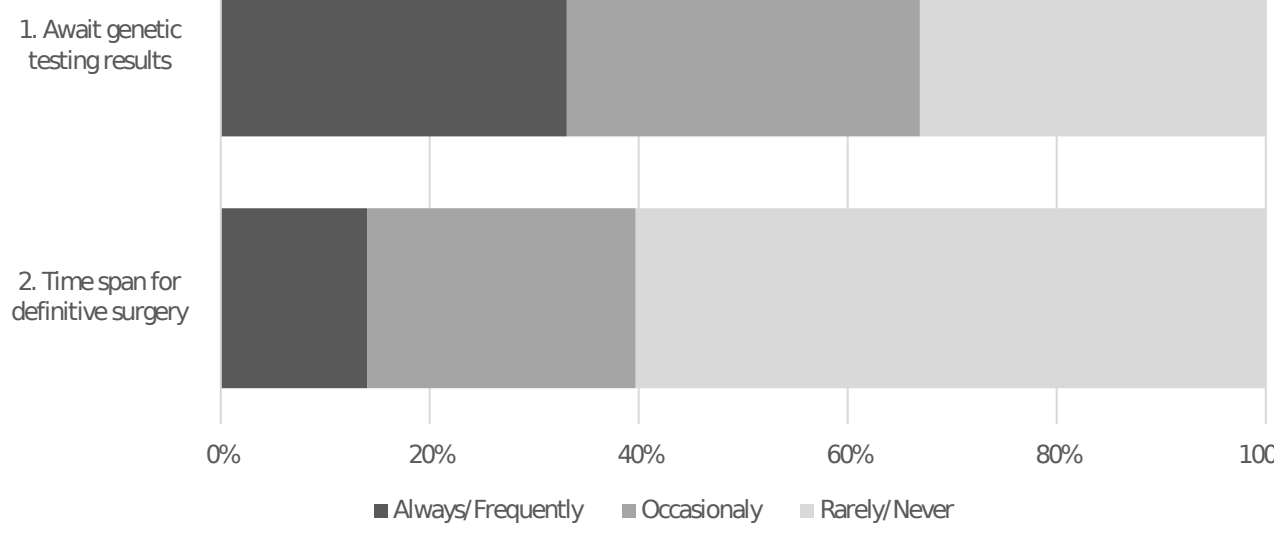

Figure 1B: Reported indications (clinical management factors) for recommending NAC 
1. Poor performance status

2. Comorbidities
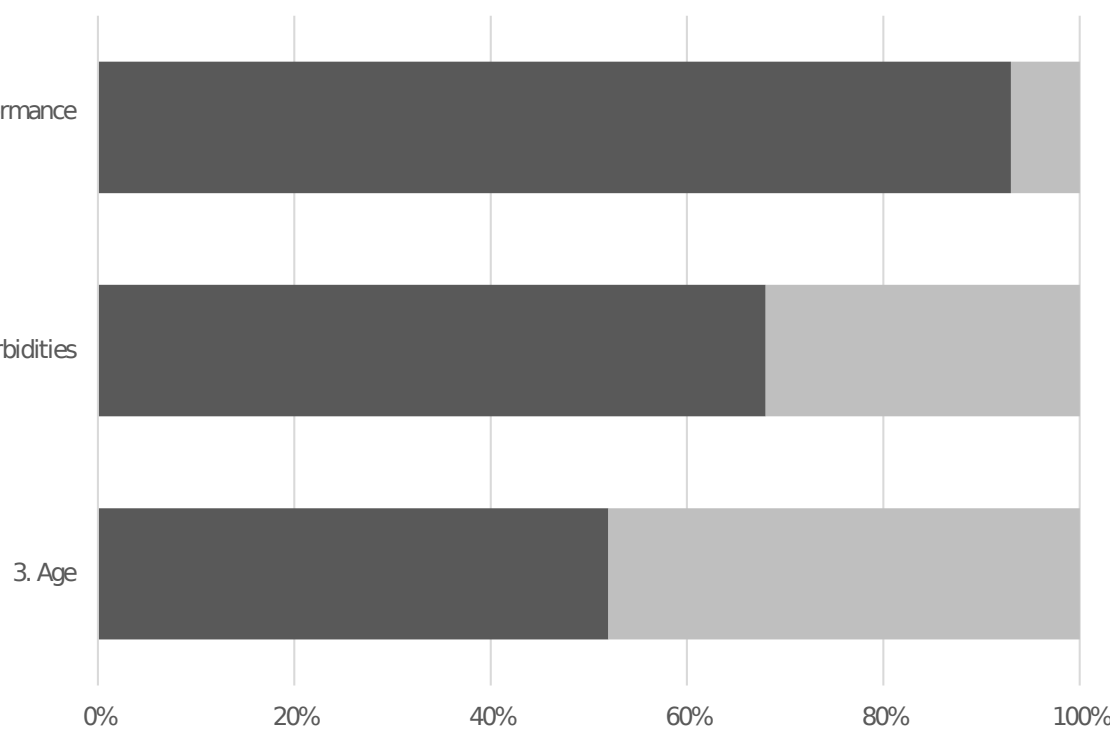

Figure 1C: Reported contraindications (patient characteristics) for recommending NAC

$60 \%$

$40 \%$

$20 \%$

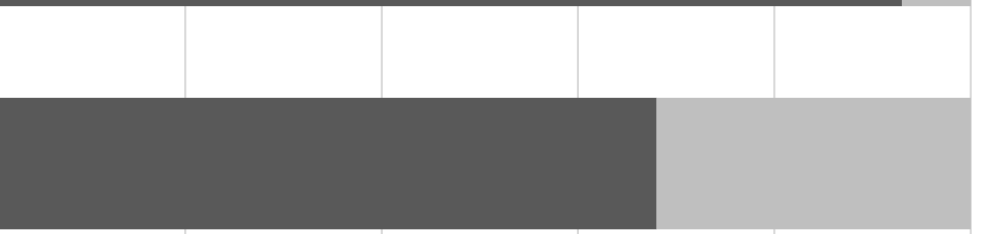

$0 \%$

$20 \%$

$40 \%$

$80 \%$

$100 \%$

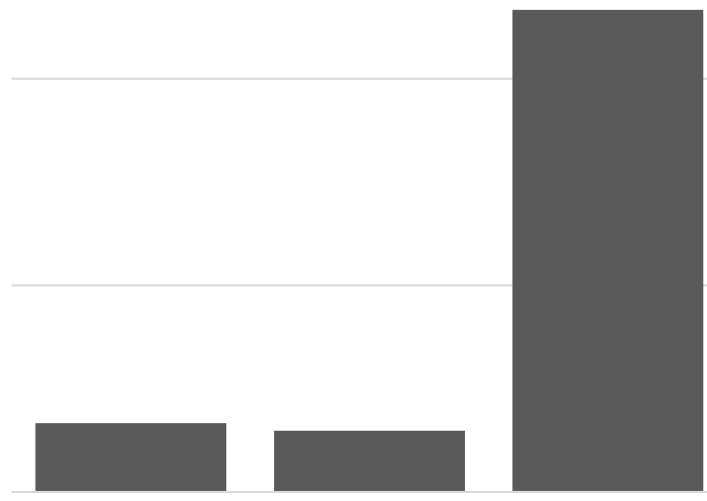

No

$$
>\text { PS } 1
$$

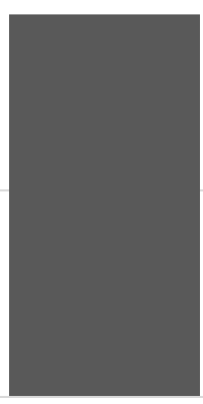

$>$ PS3

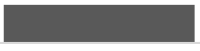

>PS4

Figure 1D: Most common reported contraindication: performance status $>2$

In the second part of the survey, clinicians were asked about other considerations that could influence the use of NAC (Table 2). More than half of the respondents (60\%), especially medical oncologist (83\%), stated that the evidence in overall and disease-free survival benefits of NAC compared to adjuvant chemotherapy is not established yet ( $p$-value: 0,015 ). While in the first part of the survey 75 percent of the respondents mentioned increased breast conservation rate as an 
indication for NAC, a concern about non-radical resections is raised by $21 \%$ of the respondents (surgeons $29,2 \%$, medical oncologists $15,8 \%$, p-value:0.078). A minor consideration in performing surgery after NAC was the increased chance of surgical complications (9\%). Finally, in a relative high percentage of clinicians (63\%), NAC is also being used to enable axillary conserving surgery.

Table 2: Agreement with statements on NAC by responding surgeons and medical oncologists

\begin{tabular}{|c|c|c|c|c|}
\hline Statements & YES & $\begin{array}{l}\text { Surgeons } \\
(\mathrm{N}=70)\end{array}$ & $\begin{array}{l}\text { Oncologists } \\
(\mathrm{N}=68)\end{array}$ & P-value \\
\hline $\begin{array}{l}\text { "NAC improves the chance of achieving axillary conservation } \\
\text { surgery" }\end{array}$ & $63 \%$ & $70.8 \%(46)$ & $62.9 \%(39)$ & 0.346 \\
\hline "NAC increases the risk of surgical complications" & $9 \%$ & $13.3 \%(8)$ & $6.9 \%(4)$ & 0.247 \\
\hline $\begin{array}{l}\text { "Breast conservation surgery after NAC increases the risk of a non- } \\
\text { radical resection" }\end{array}$ & $21 \%$ & $29.2 \%(19)$ & $15.8 \%(9)$ & 0.078 \\
\hline $\begin{array}{l}\text { "Breast conservation surgery after NAC increases the risk of } \\
\text { recurrence" }\end{array}$ & $5 \%$ & $6.5 \%(4)$ & $4.8 \%(3)$ & 0.697 \\
\hline $\begin{array}{l}\text { "There is no evidence for an overall and disease-free survival } \\
\text { benefit of NAC compared to AC" }\end{array}$ & $60 \%$ & $62.3 \%(33)$ & $82.8 \%(48)$ & 0.015 \\
\hline
\end{tabular}

In added comments, a frequently described benefit of neoadjuvant therapy was the extra time for patient work-up for surgery, for example in case of controlling diabetes or smoking cessation. Reported barriers for recommending NAC were lack of patient cooperation, logistic challenges (for example a far travel distance to the hospital), a term pregnancy, oocyte preservation, or a patient's desire to undergo surgery first.

\section{Discussion}

This survey depicts the opinion of 138 Dutch surgical and medical oncologists from 64 out of 92 hospitals in the Netherlands on NAC in BC. Despite an international trend of increasing implementation for NAC in patients with early $B C$ and the relatively high standard of care in the Netherlands, considerable variation in the use of NAC still exists between hospitals.

Respondents rated $L A B C$ as the most distinguished indication for NAC, in accordance with Dutch and international breast cancer guidelines.12 In addition, the St. Gallen Breast Cancer Conference, that focuses exclusively on the primary therapy of early breast cancer, recommends to consider NAC based on tumour biology $[14,15]$ Our survey demonstrates that only $64 \%$ of clinicians recommends NAC instead of adjuvant chemotherapy when systemic therapy is indicated based on tumour biology. The actual NAC use is even lower based on NBCA-data ( $40 \%$ in BC stage II). With the increased evidence that subgroups of patients that achieve pCR after NAC do have a better prognosis in terms of disease-free and overall survival, NAC should nowadays be considered as a preferred option in the treatment of high risk triple negative $B C$ and HER2 $B C[3,4,16]$. 
Another commonly accepted indication for NAC - confirmed by our survey - is to increase the chance of breast conservation surgery (BCS) without compromising the local recurrence rate. The ESMO guidelines on primary breast cancer advice primary systemic therapy in locally advanced and large operable cancers to allow for achieving operability or decreasing the extent of surgery [17]. In our survey, $75 \%$ of respondents recommend NAC to enable BCS. Contradictory, a relatively high percentage of $21 \%$ of respondents argued that BCS after NAC increases the risk of non-radical (i.e. resection with positive margins) resections. The restraint to use NAC to enable BCS may arise from the challenge for surgeons to determine the extent and original location of the residual lesion after NAC. More recently than our survey, a nationwide Dutch pathology study showed tumorinvolved margins in $24.3 \%$ patients after BCS after NAC, compared to $10,3 \%$ after primary BSC [18]. According to Dutch National guidelines, a tumor-free margin is defined as the absence of tumor cells at the inked margins. Although surgical experiences have been improved by the introduction of iodine-125 seeds and ultrasound guided surgery, monitoring and localization techniques are still under research [19]. It is likely that clinicians' decisions towards NAC are mainly driven by surgical management goals, rather than tumour biology and survival.

Other incentives to consider NAC, such as time necessary for testing on hereditary breast cancer, are less frequently denominated as indication of importance. Only $33 \%$ of the clinicians recommends NAC to await genetic testing results, while the discovery of a BRCA1/2 mutation may influence treatment strategies. Also, extra time for patient work-up to plan the final type of reconstructive surgery is less frequently considered important. However, NAC has the potential for improving cosmetic outcomes in oncoplastic surgery [20]. Another important consideration described by clinicians in favour of chemotherapy prior to breast surgery is the possibility to asses anti-tumour activity and clinical efficacy of new treatments in neoadjuvant chemotherapy trials [21].

The survey also revealed concerns that prevented clinicians from recommending NAC. A patients' WHO-performance status of $\geq 2$ was stated most frequently as reason to refrain from NAC, rather than advanced age. This is consistent with the idea that older patients, when selected correctly, can be treated safely with chemotherapy and that age only is no reason to refrain [22].

Although it can be questioned if these 138 experts represent the major opinion of NAC for breast cancer in the Netherlands, the main strength of this survey is that the respondents reflect practice preferences of 64 out of 89 Dutch hospitals: which means a $72 \%$ nationwide coverage, which stands for the treatment of almost 15.000 patients annually [10]. If this survey would be repeated, we expect same differences in opinions between experts' to be demonstrated. However, surveys rely heavily on the respondents' memory and opinion, thus bias should always be kept in mind when interpreting survey results. 


\section{Conclusion}

Considerable variation exists in expert opinions on NAC for early breast cancer. This article highlights the complexity of decision making for early breast cancer patients and it emphasizes the need for more consensus among specialists on the indications for NAC in early BC patients.

\section{Practice implications}

The results of this survey highlight the importance of dynamic updates of reliable clinical practice guidelines, to standardize and ensure medical quality and safety. In other words: not only clinicians' awareness on multiple arguments in favour of the use of NAC could be improved, but also the sharing of considerations and experiences - as this brief report detailing clinical practices of Dutch surgical and medical oncologists - will speed up and clarify the implementation of NAC in early breast cancer. Ultimately, it is important that patients receive unambiguous and evidencebased treatment information in order to take part in a useful process of shared decision-making. The authors do not necessarily advocate that every patient should receive NAC; however, every patient eligible to NAC should receive a choice in chemotherapy timing. Another work by our group describes how patients perceived the choice in chemotherapy timing [23]. 


\section{References}

1. Netherlands Comprehensive Cancer Organisation (IKNL). National guideline on Breast Cancer. Netherlands Comprehensive Cancer Organisation (IKNL); 2012.

2. Holmes D, Colfry A, Czerniecki B, Dickson-Witmer D, Francisco Espinel C, Feldman E, et al. Performance and Practice Guideline for the Use of Neoadjuvant Systemic Therapy in the Management of Breast Cancer. Ann Surg Oncol 2015;22(10):3184-90.

3. Von Minckwitz G, Untch M, Nüesch E, Loibl S, Kaufmann M, Kümmel S, et al. Impact of treatment characteristics on response of different breast cancer phenotypes: Pooled analysis of the German neoadjuvant chemotherapy trials. Breast Cancer Res Treat 2011;125(1):145-56.

4. Cortazar P, Zhang L, Untch M, Mehta K, Costantino JP, Wolmark N, et al. Pathological complete response and long-term clinical benefit in breast cancer: the CTNeoBC pooled analysis. Lancet 2014;384(9938):164-72.

5. Barranger E, Antomarchi J, Chamorey E, Cavrot C, Flipo B, Follana P, et al. Effect of Neoadjuvant Chemotherapy on the Surgical Treatment of Patients with Locally Advanced Breast Cancer Requiring Initial Mastectomy. Clin Breast Cancer 2015;15(5):1-5.

6. Berruti A, Brizzi MP, Generali D, Ardine M, Dogliotti L, Bruzzi P, et al. Presurgical systemic treatment of nonmetastatic breast cancer: facts and open questions. Oncologist 2008;13(11):1137-48.

7. Mougalian SS, Soulos PR, Killelea BK, Lannin DR, Abu-Khalaf MM, DiGiovanna MP, et al. Use of neoadjuvant chemotherapy for patients with stage I to III breast cancer in the United States. Cancer 2015;121(15):254452.

8. van Bommel ACM, Spronk PER, Vrancken Peeters MJTFD, et al. Clinical auditing as an instrument for quality improvement in breast cancer care in the Netherlands: The national NABON Breast Cancer Audit. J Surg Oncol 2016:1-7.

9. Spronk PER, van Bommel ACM, Smorenburg CH, Jager A, Lobbes M, Maduro JH, et al. Variation in use of neoadjuvant chemotherapy in patients with stage III breast cancer: results of the Dutch national breast cancer audit. [submitted]

10. DICA annual rapport. Available at: https://www.dica.nl/jaarrapportage-2014/nbca

11. Kunneman M, Engelhardt EG, Hove FLL, Marijnen CA, Portielje JE, Smets EM, et al. Deciding about (neo-) adjuvant rectal and breast cancer treatment : Missed opportunities for shared decision making. Acta Oncol 2016;55:134-9.

12. Wei JP, Sherry RM, Baisden BL, Peckel J, Lala G. Prospective hospital-based survey of attitudes of Southern women toward surgical treatment of breast cancer. Ann Surg Oncol 1995;2(4):360-4.

13. Caldon LJM, Walters SJ, Ratcliffe J, Reed MWR, Sheffield S. What influences clinicians' operative preferences for women with breast cancer? An application of the discrete choice experiment. Eur J Cancer 2007 Jul;43(11):1662-9.

14. Gnant M, Thomssen C, Harbeck N. St. Gallen/Vienna 2015: A brief summary of the consensus discussion. Breast Care 2015;10(2):124-30.

15. Onclive 2017. Available at: http://www.onclive.com/conference-coverage/st-gallen 2017/neoadjuvanttherapy fundamental-in-tnbc-and-her2-breast-cancer

16. Cortazar P, Geyer CE Jr., Pathological Complete Response in Neoadjuvant Treatment of Breast Cancer. Ann Surg Oncol. 2015;22(5):1441-6.

17. Cardoso F, Costa A, Senkus E, Aapro M, André F, Barrios CH, et al. 3rd ESO-ESMO international consensus guidelines for Advanced Breast Cancer (ABC 3). Ann Oncol. 2017;31:244-59.

18. Volders JH, Haloua MH, Krekel NMA. Neoadjuvant chemotherapy in breast-conserving surgery consequences on margin status and excision volumes: A nationwide pathology study. Eur J Surg Oncol 2016 Jul;42(7):986-93.

19. Hayes MK. Update on preoperative breast localization. Radiol Clin North Am 2017 May;55(3):591-603.

20. Jagsi R, Li Y, Morrow M, Janz N, Alderman A, Graff J, et al. Patient-reported Quality of Life and Satisfaction With Cosmetic Outcomes After Breast Conservation and Mastectomy With and Without Reconstruction. Ann Surg 2015;261(6):1198-206. 
21. Esserman LJ, Berry DA, DeMichele A, Carey L, Davis SE, Buxton M, et al. Pathologic complete response predicts recurrence-free survival more effectively by cancer subset: results from the I-SPY 1 TRIAL--CALGB 150007/150012, ACRIN 6657. J Clin Oncol 2012 Sep 10;30(26):3242-9.

22. Heijmen L, van Laarhoven HW, Punt CJ, van den Hurk D, van der Drift MA, Ottevanger PB, et al. Encouraging results in older patients receiving chemotherapy: A retrospective analysis of treatment guideline adherence in daily practice. Geriatr Gerontol Int 2012;12(1):80-5.

23. de Ligt KM, Spronk PER, van Bommel ACM, Vrancken Peeters MTFD, Siesling S, Smorenburg CH. Patients' experiences with decisions on timing of chemotherapy for breast cancer. Breast 2018;37:99-106. 
Supplement 1: 20-question survey on the influence of patient, disease, and management related factors on decisions towards NAC.

\section{General Information}

1. What institute do you work for?

2. Are you working in an affiliated or other institute as well?

3. Sex $\mathrm{m} / \mathrm{v}$

4. Age

5. Specialism: surgeon / medical oncologist / other

6. Number of years in practice in current specialism (training excluded)

7. Number of new patients diagnosed with breast cancer treated per year

\section{Diagnostics}

8. Are the following diagnostic modalities typically applied prior to the commence of neoadjuvant chemotherapy (NAC)?

- MRI

- $\quad$ PET-CT

$$
\begin{aligned}
& \text { Never - Rarely - Sometimes - Often - Always } \\
& \text { Never - Rarely - Sometimes - Often - Always }
\end{aligned}
$$

Add comments

PART I. Indications and contraindications of NAC

9. Which items do you consider to be indications for the use of neoadjuvant chemotherapy (NAC)?

- $\quad$ Locally advanced disease (stage III)

- Downstage to breast conserving surgery

Never - Rarely - Sometimes - Often - Always

- Any adjuvant chemotherapy candidate / systemic therapy is indicated preoperatively

Never - Rarely - Sometimes - Often - Always

- $\quad$ Await genetic testing results

- $\quad$ Time span for definitive surgery

Never - Rarely - Sometimes - Often - Always

Never - Rarely - Sometimes - Often - Always

Other/ add comments

10. Other / missing indications?

11. Ranking from $1-6$ (most - less important) indication for neoadjuvant chemotherapy (NAC):

Locally advanced disease (stage III)

Downstage to breast conserving surgery

Any adjuvant chemotherapy candidate /systemic therapy is indicated preoperatively

Awaiting genetic testing results

Time span for definitive surgery

12. Do you consider age to be a contraindication for the use of neoadjuvant chemotherapy (NAC)?

No, age alone is no contraindication

Yes, for patients aged $<55$

Yes, for patients aged $<60$

Yes, for patients aged $<65$

Yes, for patients aged $<70$

Yes, for patients aged $<75$ 
13. Do you consider the presence of comorbidities to be a contraindication for the use of neoadjuvant chemotherapy (NAC)?

According to the Charlson Index Scale:

No, comorbidities are no contraindication

Yes, for cardiac disease

Yes, for vascular disease

Yes, for pulmonary disease

Yes, for gastrointestinal disease

Yes, for urogenital disease

Yes, for thrombotic disease

Yes, for muscle and joint disease

Yes, for endocrine system disease

Other/ add comments

14. Do you consider a poor performance status (PS) to be a contraindication for the use of neoadjuvant chemotherapy (NAC)?

According to the ECOG/WHO Performance Scale:

No, a poor performance status is no contraindication

Yes, if PS=0 - Asymptomatic (Fully active, able to carry on all predisease activities without restriction)

Yes, if PS=1 - Symptomatic but completely ambulatory (Restricted in physically strenuous activity but ambulatory and able to carry out work of a light or sedentary nature. For example, light housework, office work)

Yes, if PS=2 - Symptomatic, $<50 \%$ in bed during the day (Ambulatory and capable of all self care but unable to carry out any work activities. Up and about more than $50 \%$ of waking hours)

Yes, if PS $=3$ - Symptomatic, $>50 \%$ in bed, but not bedbound (Capable of only limited self-care, confined to bed or chair $50 \%$ or more of waking hours)

Yes, if PS=4 - Bedbound (Completely disabled. Cannot carry on any self-care. Totally confined to bed or chair)

15. Ranking from $1-3$ (most - less important) contraindication:

High age

Comorbidities

Poor performance status

16. Other / missing contraindications?

Part II. Other considerations that could influence the use of NAC

17. To what extent do you agree or disagree with the following statements?

- "NAC improves the chance of achieving axillary conservation surgery"

Agree/Disagree

- "NAC increases the risk of surgical complications"

Agree/Disagree

- "Breast conservation surgery after NAC increases the risk of a non-radical resection"

- "Breast conservation surgery after NAC increases the risk of recurrence"

Agree/Disagree

Agree/Disagree

- $\quad$ "There is no evidence for an overall and disease-free survival benefit of NAC compared to $\mathrm{AC}^{\prime \prime}$

Agree/Disagree 


\section{Add comments}

\section{Final section about study participation and interests}

18. Number of new patients included in trials a year (national and international $\quad>10$ or $<10$ level)

Other/add comments

19. Do you visit one of the following conferences on a regular base?

\section{SABCS}

Bossche mammadagen (Dutch conference - annual conference for breast surgeons and medical oncologists)

\section{EBCC}

St. Gallen

Chirurgendagen (Dutch conference - annual conference for surgeons in general)

No, I never visit one of these conferences

Other/add comments

20. Possibility to add any questions or comments 



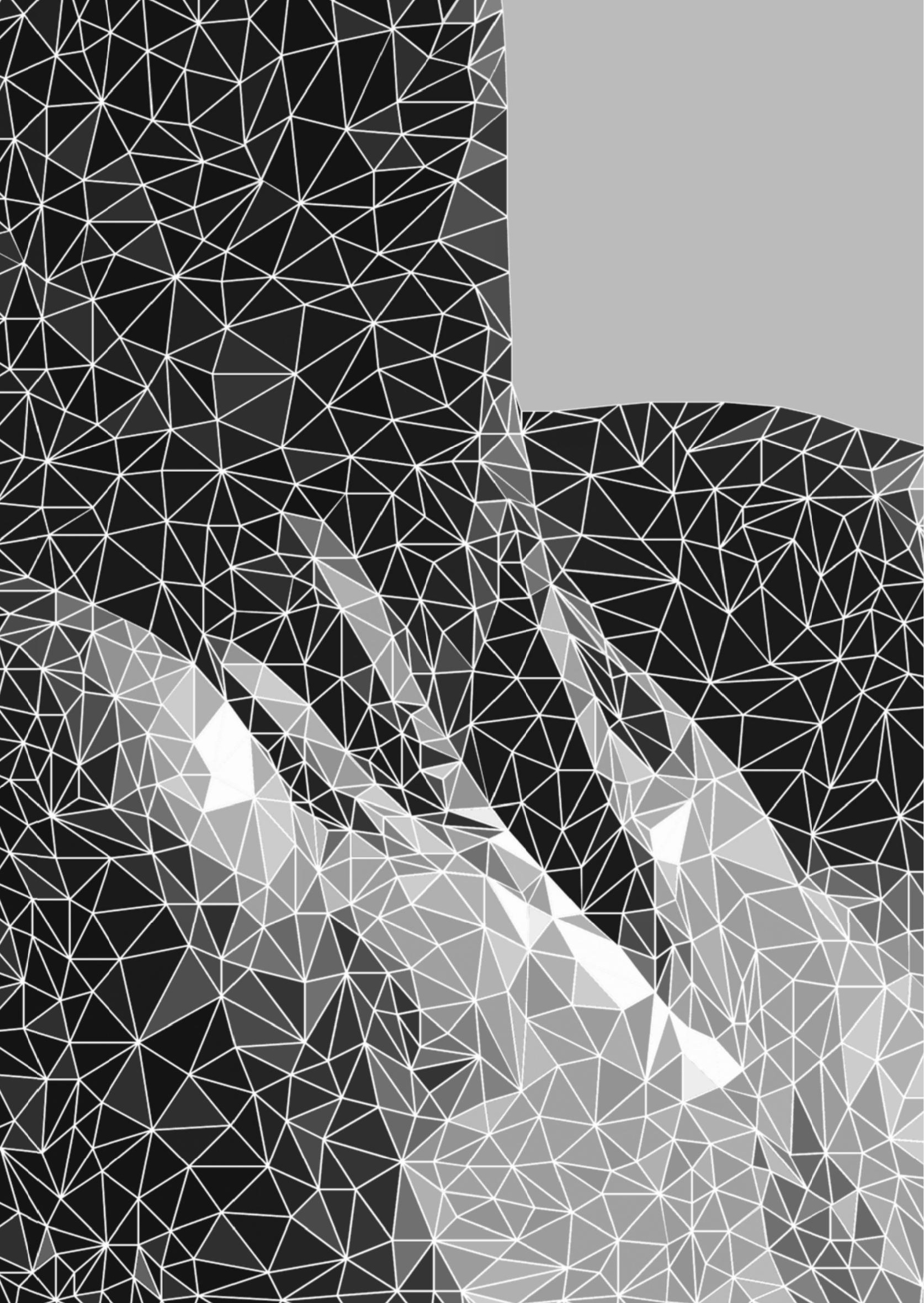


SECOND PART

DECISIONS ABOUT BREAST RECONSTRUCTION 


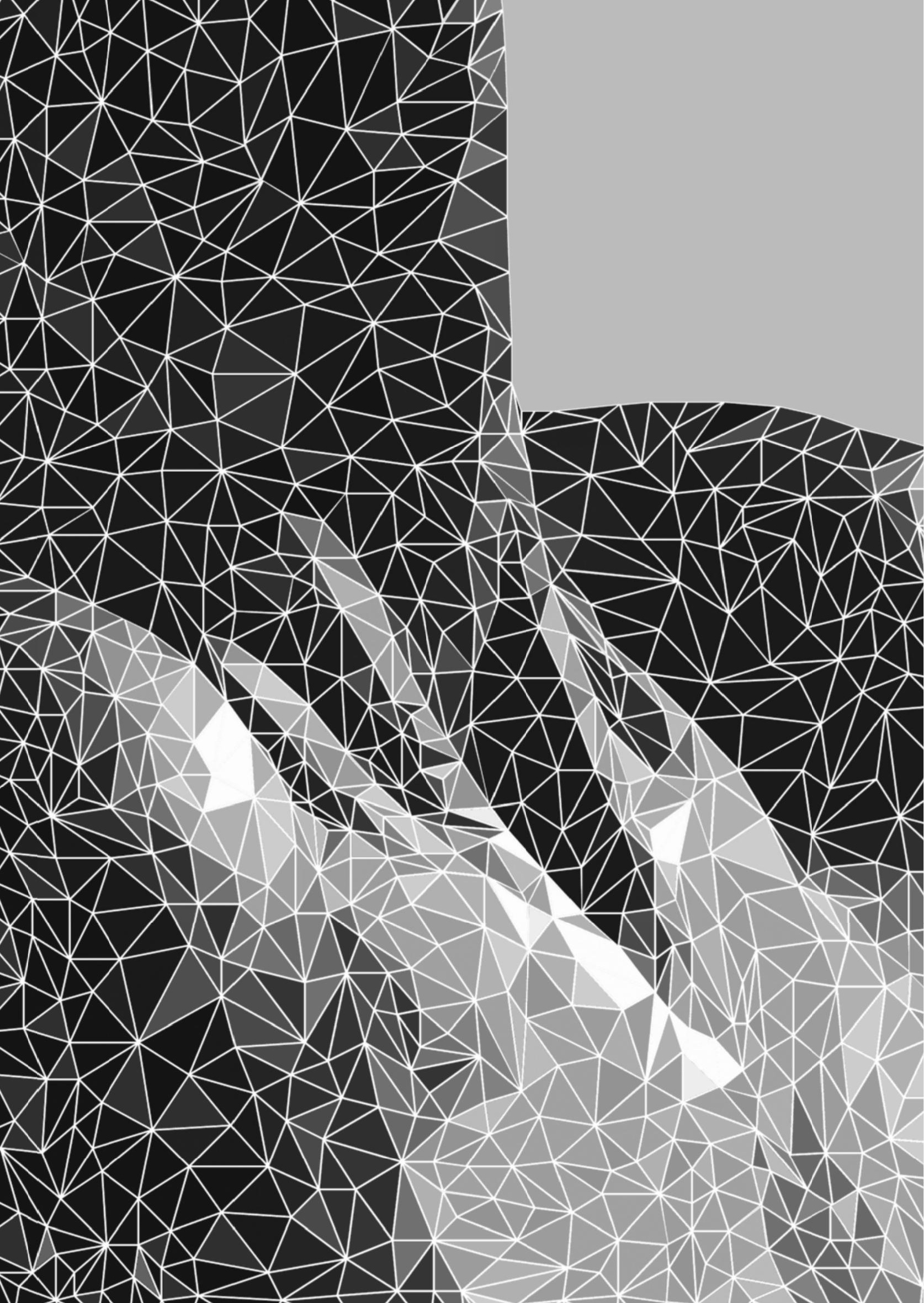




\section{CHAPTER 3}

LONG-TERM HEALTH-RELATED QUALITY OF LIFE AFTER FOUR COMMON SURGICAL TREATMENT OPTIONS FOR BREAST CANCER AND THE EFFECT OF COMPLICATIONS - A RETROSPECTIVE PATIENTREPORTED SURVEY AMONG 1871 PATIENTS

C.A.E. Kouwenberg* K.M. de Ligt*

L.W. Kranenburg H. Rakhorst

D. de Leeuw

S. Siesling J.J. Busschbach M.A.M. Mureau

*contributed equally

Submitted, March 2019 



\section{Abstract}

\section{Background:}

Differences in health-related quality of life (HRQoL)-outcomes after different surgical breast cancer treatment options, including breast reconstruction (BR), are relevant for counseling individual patients in clinical decision-making, and for (societal) evaluations such as (cost-) effectiveness analyses. However, current literature shows contradictory results, due to use of different PatientReported Outcome Measures (PROMs) and study designs with limited patient numbers. Therefore, this article sets out to improve this evidence from a clinical and societal perspective using PROMs in a large, cross-sectional study for different surgical breast cancer treatment options.

\section{Methods:}

HRQoL was assessed through the EQ-5D-5L, EORTC-QLQ-C30/-BR23 and Breast-Q. Patients with different treatments (breast conserving surgery (BCS), mastectomy (MAS), autologous BR (A-BR), implant-based BR (I-BR)) were compared after propensity-weighted adjustment of pre-treatment differences. The EQ-5D was used to value the effect of surgical complications.

\section{Results:}

1871 breast cancer patients participated (BCS: $n=615$, MAS: $n=507, A-B R: n=330,1-B R: n=419$ ). MAS-patients reported the lowest mean HRQoL based on the EQ-5D (MAS:0.805, BCS:0.844, A-BR:0.849, I-BR:0.850) and functioning scores of the EORTC-QLQ-C30. Based on the Breast-Q, A-BR-patients had higher mean satisfaction with outcome and satisfaction with breasts and had higher sexual well-being scores than I-BR patients. Patients with complications (except for I-BR) reported statistically significant lower HRQoL than patients without complications. Complications in A-BR patients resulted in a substantially lower HRQoL than in I-BR patients.

\section{Conclusions:}

Our study shows the added value of breast conservation and reconstruction compared to mastectomy, however, differences between BCS, I-BR, and A-BR were subtle. Complications resulted in poorer HRQoL. 


\section{Introduction}

Because five-year survival rates for early stage breast cancer are relatively high [1, 2], the effects of breast cancer and its treatment on quality of life become more important, which may affect surgical decision-making. Since mastectomy and breast conserving surgery (BCS) including radiotherapy have similar disease-free and overall survival [3-5], the effects of different treatment modalities on outcomes other than survival gain significance. Because loss of a breast may negatively affect psychosocial health, body image, and sexual function [6], guidelines recommend that the possibility of breast reconstruction (BR) should be discussed with every patient scheduled for MAS [7-9]. Multiple options are available, either using autologous tissue (autologous BR, A-BR) or breast implants (implant BR, I-BR), varying in costs, timing, duration, complication rates, and cosmetic results $[6,10,11]$. BR aims to improve the patient's well-being and health-related quality of life (HRQoL), $[6,10]$ but patients opting for BR also have a risk of complications [12-15], reconstruction failure [12, 13], or disappointing (cosmetic) results [6]. Consequently, shared decision-making (SDM) between physicians and patients about the preferred surgical treatment is a complex tradeoff between outcomes and risks.

HRQoL-outcomes after different surgical breast cancer treatment options are relevant for counseling individual patients in clinical decision-making, and for societal evaluations as costeffectiveness analyses used in health policy. Research shows that post-treatment HRQoL is relatively high in breast cancer patients, but evidence about (differences in) HRQoL after different treatment options is conflicting [16-19]. This conflicting evidence may be explained by variation in the use of patient-reported outcome measures (PROMs), study designs, and patient populations. For instance, there are both studies that have and have not found differences in HRQoL between patients who had undergone BCS or MAS [20]. Also, several higher quality studies did not find statistically significant differences in HRQoL, body image, and sexuality between patients with or without BR [21]. We believe that evidence should be improved, as such information is relevant for choosing a treatment in clinical decision-making and for health policy. Until now, outcomes have been generally measured in small, cross-sectional, mono-center studies. Ideally, one would include all surgical options relevant to breast cancer patients in one large prospective cohort study [21]. Santosa et al. performed such a large prospective study, comparing patients with I-BR and A-BR [22]. Furthermore, outcomes measured over a longer period of time would be of interest, as different surgical outcomes may have a different HRQoL-course over time. For example, recovery from surgical complications will take additional time.

To improve the evidence on the impact of breast cancer surgery and consequently for clinical decision-making and health policy, the present study aimed to compare HRQoL outcomes for four common surgical breast cancer treatment options (BSC, MAS, A-BR, I-BR). HRQoL was assessed using multiple PROMs in a large, multicenter, retrospective, cross-sectional cohort of breast cancer patients up to ten years after diagnosis. The second aim was to investigate the impact of complications on HRQoL following these different surgical treatment options. We hypothesized that BCS and A-BR are favorable over I-BR and MAS in terms of HRQoL, however, in the absence of complications, with MAS yielding the least preferable outcomes. 


\section{Methods}

\section{Study population}

Female breast cancer patients $(n=3,804)$ from four hospitals in the Netherlands (one academic hospital, three general hospitals) were invited by mail to participate in a self-administered cross-sectional online survey. Patients were included if they had been surgically treated for nonmetastatic breast cancer in the last ten years (2008-2018). Patients who had developed distant metastases since curative treatment or who were not proficient in Dutch were excluded. Four groups were formed based on the surgical procedure: BCS, MAS, A-BR, or I-BR. Time between surgery and invitation was over 6 months, to ensure patients had recovered from the treatment. Patients who preferred completing a paper questionnaire, were sent one on request. Respondents gave informed consent for processing their coded survey results. The Medical Ethics Committee of the Erasmus MC reviewed and approved the study protocol (MEC-2015-273).

\section{Measures}

The survey included questions regarding baseline patient and treatment characteristics, including surgical complications, and the following validated questionnaires:

EQ-5D-5L. This questionnaire of health status measures problems in five dimensions (mobility, self-care, usual activities, pain/discomfort, and anxiety/depression), all with five levels of severity (no, slight, moderate, severe, and extreme problems). Per health state, a 'value' can be assigned, where 0.00 and 1.00 represent the value for death and perfect health, respectively. This 'value' is also referred to as utility, index score, or preference. The EQ-5D includes values of the general public in the valuation of HRQoL, resulting in 'preference-weighted QoL scores', often referred to as 'utilities'. Utilities are used as outcomes in economic evaluations, which can inform health policy. A mean general population (GP) reference was obtained to compare study results to that of a sample of the Dutch general population [23].

EORTC QLQ-C30/BR23. The EORTC Quality of Life Questionnaires (QLQ) measures HRQoL in cancer patients [24], the breast cancer-specific QLQ-BR23 supplements the cancer-specific QLQ-C30 [25]. Both consist of functioning and symptom scales; the QLQ-C30 also includes a global health status scale. All items and scales range from 0 to 100 , with higher scores presenting a higher level of functioning or general health for the functional and global health status scales, respectively, and higher scores representing a higher level of symptoms for the symptom scales [26].

Breast-Q. A treatment-specific PROM, developed to measure breast-related QoL and satisfaction, on several HRQoL domains. Six postoperative domains were used from the different modules which were developed for the respective patient groups: 'Satisfaction with Breasts', 'Satisfaction with Outcome', 'Psychosocial Well-being', 'Sexual Well-being', 'Physical Well-being Chest', 'Physical Well-being Abdomen'. The raw scores of the Breast- $Q$ domains were converted to scores between 0 (worst) and 100 (best) using the Q-score software [27]. 


\section{Analyses}

Twenty-five patients did not report their highest completed education level; these missing answers were imputed using a single imputation method. A propensity-score weighting for multiple treatments was calculated according to the method of McCaffrey et al. [28] to adjust for covariates that predict receiving either one of the four surgical treatment options, thereby reducing the effects of confounding. The following clinical and sociodemographic characteristics were included in the propensity weight calculation: age at time of survey, education, year of breast cancer diagnosis, year of surgery, chemotherapy, hormone therapy, and breast cancer recurrence. The EQ-5D Dutch general population reference sample (GP) was matched to all surgical groups using age and sex as the matching variables. Propensity weights were calculated by the Toolkit for Weighting and Analysis of Non-equivalent Groups (TWANG) Package for STATA [29].

For all responding patients, propensity-adjusted patient and treatment characteristics and HRQoL were presented. Propensity weights were incorporated in the analyses using the Stata-SE14 survey (svy) post-estimation function [30]. Mean scores, confidence intervals, and pair-wise comparisons were subsequently obtained and performed using the margins regression estimation function. Column proportions were chi-squared tested. The utilities resulting from the EQ-5D per surgical treatment were stratified by experienced surgical complications. Utilities per surgical treatment were plotted over the course of time in three-year intervals $(\leq 3 ; 3 \leq 6 ; 6 \leq 9 ;>9)$, starting at time of last BR.

For statistical testing, two-sided $p$-values $\leq 0.05$ were considered statistically significant. All analyses were performed in StataCorp Stata-SE14 [31].

\section{Results}

1,871 out of 3,804 patients (49\%) responded, consisting of $615 \mathrm{BCS}, 507 \mathrm{MAS}, 330 \mathrm{~A}-\mathrm{BR}$, and $419 \mathrm{I}-\mathrm{BR}$ patients. Table 1 presents patient and treatment characteristics. Nearly all A-BR procedures were abdominally-based free-flap reconstructions. After propensity-weighted adjustment, estimated group sizes were reduced to 434.0 BCS, 386.3 MAS, 178.6 A-BR, and 295.5 I-BR patients. Group sizes declined as patients with certain characteristics from one group occurred less frequently in another group, and thus received a relative score weight lower than 1 . After propensity-weighted adjustment, balance was achieved for all variables, except for age and chemotherapy treatment. 


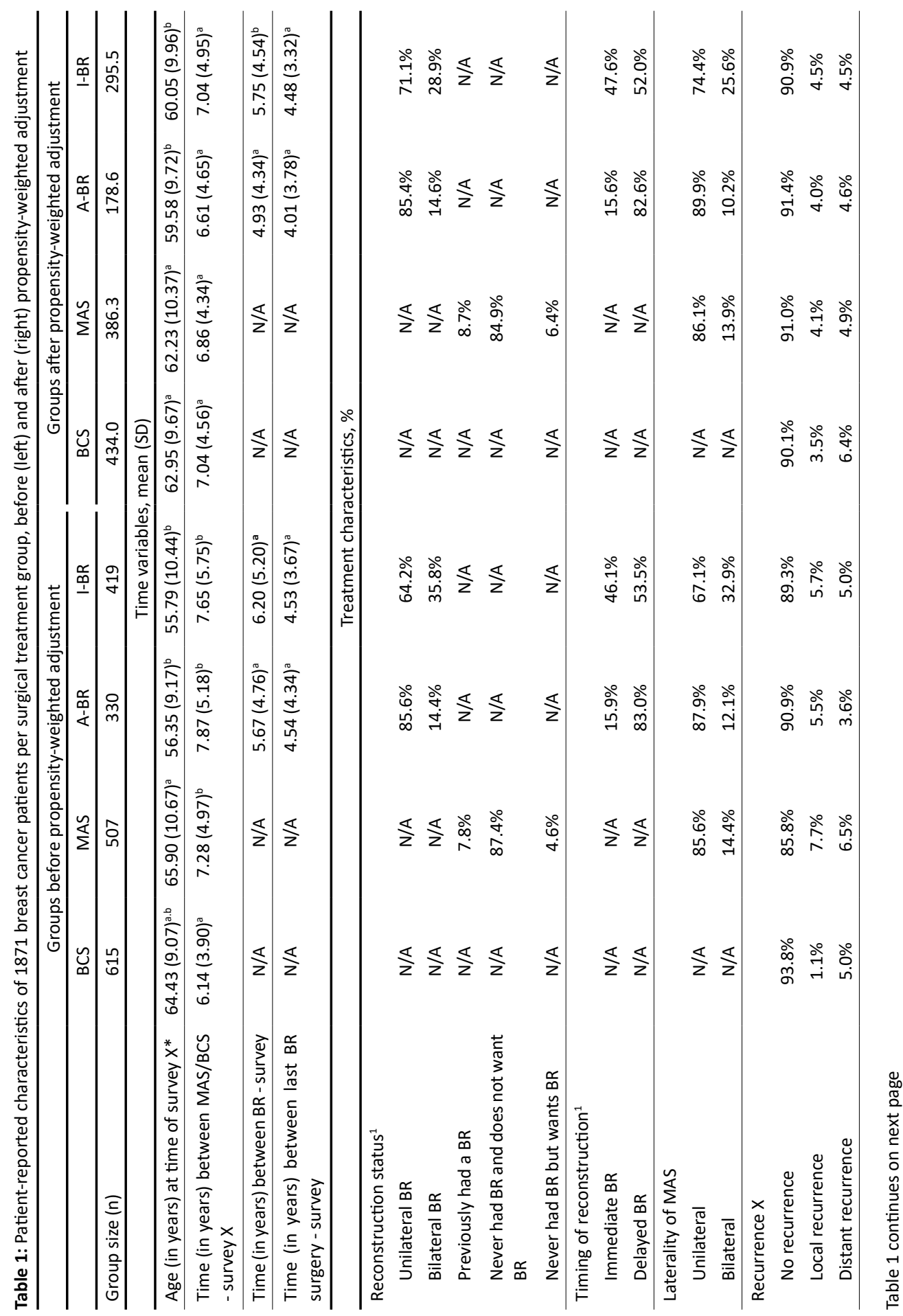




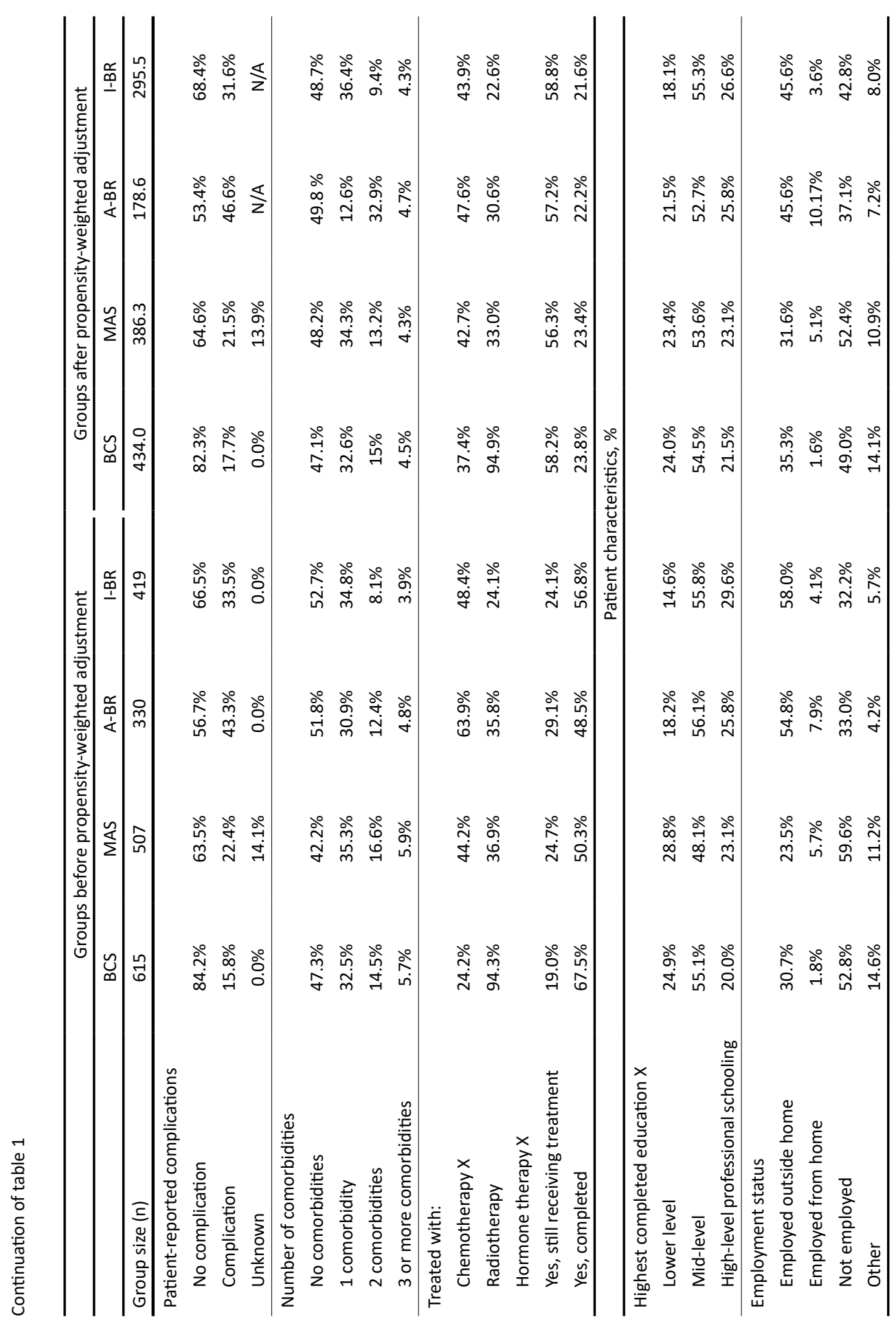




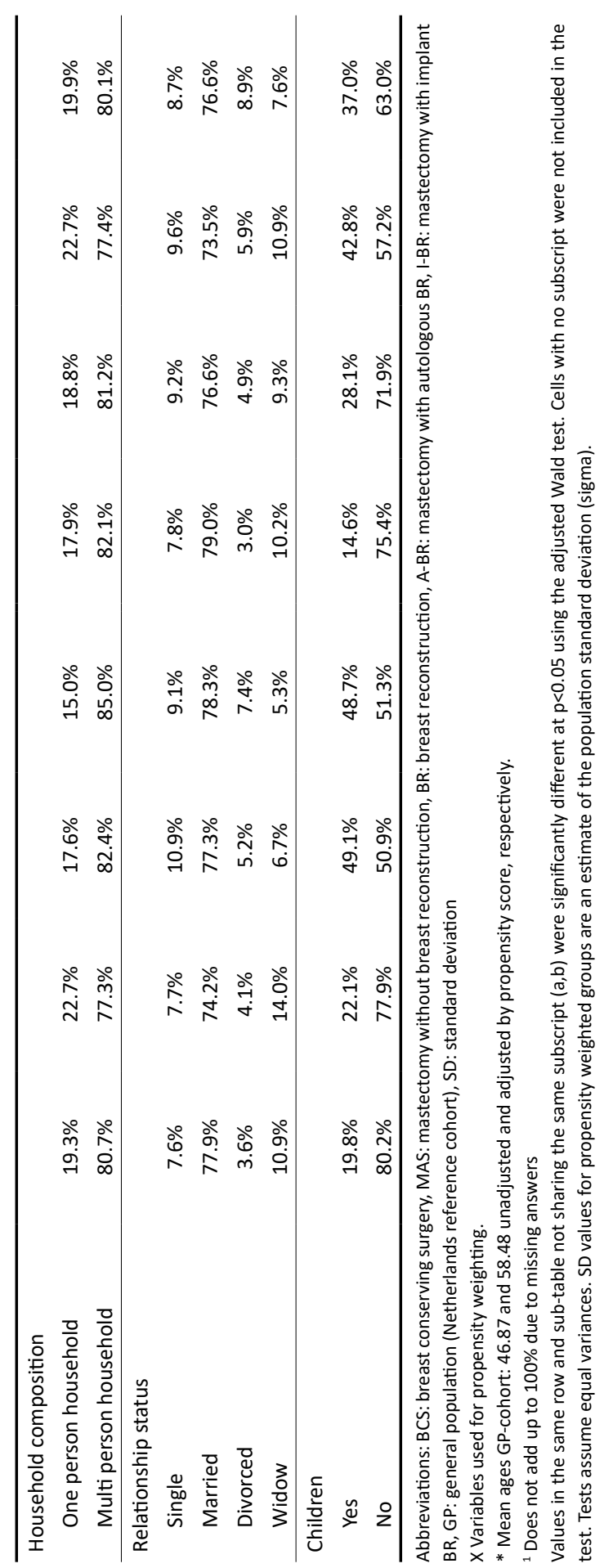




\section{Quality of life outcomes}

Table 2 presents preference-based HRQoL outcomes at time of survey per group. After propensityweighted adjustment, patients treated with MAS reported a statistically significant lower mean EQ-5D score (0.805) compared to all other surgical groups (BCS: 0.844; A-BR: 0.849; I-BR: 0.850). Pairwise comparisons of the groups for the individual EQ-5D domains reflected these lower means for MAS as well (Figure 1).

Furthermore, for two EORTC-QLQ-C30 functioning scales, statistically significant differences were found. First, patients treated with I-BR reported a statistically significant higher mean 'Physical functioning' (87.97) than patients with BCS (84.53) or MAS (82.94), although comparable to A-BR (85.62). Second, I-BR patients reported a statistically significant higher mean 'Role functioning' (86.02) compared to patients treated with MAS (80.70). Within the 'Symptom Scales', statistically significant more favorable mean scores were found for BCS over MAS for 'Pain' (BCS: 15.41, MAS: 18.93;) and 'Financial problems' (BCS: 5.23, MAS: 8.22).

Based on the EORTC-QLQ-BR23 scores, mean 'Body image' was significantly higher for BCS-patients (BCS: 87.45; MAS: 80.49; A-BR: 82.28; I-BR: 82.35). BCS-patients also reported the lowest mean 'Arm symptoms' (BCS: 12.68; MAS: 17.12; A-BR: 18.18; I-BR: 16.82). In contrast, 'Breast symptoms' on average were more often reported by patients treated with BCS (13.45) than MAS (9.94) or $A-B R$ (8.79). Patients with A-BR and I-BR reported the highest mean 'Sexual enjoyment' (A-BR: 64.24; I-BR: 63.80) compared to BCS or MAS (BCS: 57.03; MAS: 54.82).

For the Breast-Q scales, patients with A-BR reported the highest mean 'Satisfaction with Breasts' (A-BR: 71.29) compared to the other groups (BCS: 65.52; MAS: 60.65; I-BR: 59.39). Interestingly, 'Satisfaction with Breasts' for MAS and I-BR did not differ significantly. Mean 'Satisfaction with Outcome' and 'Satisfaction with Nipple' were significantly higher in A-BR than I-BR patients (ABR: 75.75 vs, I-BR: 66.37; and A-BR: 63.03; I-BR: 54.96, respectively). MAS-patients reported the lowest mean 'Psychosocial well-being' (66.50) and 'Sexual well-being' (50.00). 
Table 2: Mean patient-reported quality of life scores of 1871 breast cancer patients per surgical treatment and the Dutch general population, after propensity-weighted adjustment

\begin{tabular}{|c|c|c|c|c|c|}
\hline & \multicolumn{5}{|c|}{ Propensity weighted groups (mean, $\mathrm{Cl}$ ) } \\
\hline & BCS & MAS & A-BR & I-BR & GP \\
\hline Group size (n) & 434.0 & 386.3 & 178.6 & 295.5 & N/A \\
\hline \multicolumn{6}{|l|}{ EQ-5D-5L } \\
\hline Utilities & $\begin{array}{c}0.844^{a} \\
(0.829-0.859)\end{array}$ & $\begin{array}{c}0.805^{b} \\
(0.787-0.823)\end{array}$ & $\begin{array}{c}0.849^{a} \\
(0.828-0.871)\end{array}$ & $\begin{array}{c}0.850^{\mathrm{a}} \\
(0.823-0.877)\end{array}$ & $\begin{array}{c}0.833^{\mathrm{a}} \\
(0.812-0.854)\end{array}$ \\
\hline VAS Score & $\begin{array}{c}77.76^{a} \\
(76.02-79.51)\end{array}$ & $\begin{array}{c}76.48^{a} \\
(74.75-78.22)\end{array}$ & $\begin{array}{c}79.24^{\mathrm{a}} \\
(76.76-81.72)\end{array}$ & $\begin{array}{c}77.58^{a} \\
(75.52-79-65)\end{array}$ & $\begin{array}{c}78.88^{a} \\
(77.08-80.69)\end{array}$ \\
\hline \multicolumn{6}{|l|}{ EORTC-QLQ-C30 } \\
\hline Global Health Status & $\begin{array}{c}80.00^{a} \\
(78.26-81.75)\end{array}$ & $\begin{array}{c}79.01^{\mathrm{a}} \\
(77.14-80.87)\end{array}$ & $\begin{array}{c}81.38^{a} \\
(79.22-83.54)\end{array}$ & $\begin{array}{c}80.16^{\mathrm{a}} \\
(78.07-82.25)\end{array}$ & N/A \\
\hline \multicolumn{6}{|l|}{ Function scales: } \\
\hline Physical function & $\begin{array}{c}84.53^{a} \\
(83.02-86.04)\end{array}$ & $\begin{array}{c}82.94^{\mathrm{a}} \\
(81.32-84.55)\end{array}$ & $\begin{array}{c}85.62^{a, b} \\
(83.30-87.85)\end{array}$ & $\begin{array}{c}87.97^{b} \\
(85.63-90.31)\end{array}$ & N/A \\
\hline Role function & $\begin{array}{c}84.35^{\mathrm{a}} \\
(82.34-86.36)\end{array}$ & $\begin{array}{c}80.70^{\mathrm{b}} \\
(80.84-87.19)\end{array}$ & $\begin{array}{c}84.02^{a, b} \\
(82.82-89.22)\end{array}$ & $\begin{array}{c}86.02^{\mathrm{a}} \\
(82.82-89.22)\end{array}$ & N/A \\
\hline Emotional function & $\begin{array}{c}85.36^{a} \\
(83.36-87.18)\end{array}$ & $\begin{array}{c}83.84^{a} \\
(81.90-85.78)\end{array}$ & $\begin{array}{c}87.00^{\mathrm{a}} \\
(84.47-89.51)\end{array}$ & $\begin{array}{c}85.04^{a} \\
(82.40-87.67)\end{array}$ & N/A \\
\hline Cognitive function & $\begin{array}{c}84.14^{a} \\
(82.21-86.06)\end{array}$ & $\begin{array}{c}84.32^{\mathrm{a}} \\
(82.32-86.31)\end{array}$ & $\begin{array}{c}83.67^{a} \\
(80.53-86.82)\end{array}$ & $\begin{array}{c}84.97^{a} \\
(82.29-87.65)\end{array}$ & N/A \\
\hline Social function & $\begin{array}{c}88.82^{\mathrm{a}} \\
(87.01-90.63)\end{array}$ & $\begin{array}{c}86.94^{a} \\
(87.01-90.63)\end{array}$ & $\begin{array}{c}88.02^{\mathrm{a}} \\
(85.54-90.50)\end{array}$ & $\begin{array}{c}87.48^{a} \\
(84.40-90.55)\end{array}$ & N/A \\
\hline \multicolumn{6}{|l|}{ Symptom scales: } \\
\hline Fatigue & $\begin{array}{c}21.82^{\mathrm{a}} \\
(19.77-23.87)\end{array}$ & $\begin{array}{c}22.54^{a} \\
(20.30-24.77)\end{array}$ & $\begin{array}{c}22.39^{a} \\
(19.27-25.51)\end{array}$ & $\begin{array}{c}20.21^{\mathrm{a}} \\
(17.47-22.94)\end{array}$ & N/A \\
\hline Nausea and vomiting & $\begin{array}{c}2.72^{\mathrm{a}} \\
(1.98-3.46)\end{array}$ & $\begin{array}{c}3.31^{\mathrm{a}} \\
(2.25-4.38)\end{array}$ & $\begin{array}{c}2.83^{\mathrm{a}} \\
(1.76-3.90)\end{array}$ & $\begin{array}{c}3.35^{\mathrm{a}} \\
(1.40-5.30)\end{array}$ & N/A \\
\hline Pain & $\begin{array}{c}15.41^{\mathrm{a}} \\
(13.56-17.25)\end{array}$ & $\begin{array}{c}18.93^{b} \\
(16.53-21.32)\end{array}$ & $\begin{array}{c}17.18^{\mathrm{a}, \mathrm{b}} \\
(14.07-20.28)\end{array}$ & $\begin{array}{c}15.89^{a, b} \\
12.87-18.90)\end{array}$ & N/A \\
\hline Dyspnea & $\begin{array}{c}14.28^{a} \\
(12.18-16.39)\end{array}$ & $\begin{array}{c}12.22^{\mathrm{a}} \\
(10.12-14.31)\end{array}$ & $\begin{array}{c}13.39^{a} \\
(9.75-17.03)\end{array}$ & $\begin{array}{c}10.82^{\mathrm{a}} \\
(8.06-13.59)\end{array}$ & $\mathrm{N} / \mathrm{A}$ \\
\hline Insomnia & $\begin{array}{c}22.76^{a} \\
(20.09-25.44)\end{array}$ & $\begin{array}{c}22.69^{a} \\
(19.93-25.44)\end{array}$ & $\begin{array}{c}20.37^{a} \\
(16.87-23.87)\end{array}$ & $\begin{array}{c}25.11^{\mathrm{a}} \\
(21.10-29.11)\end{array}$ & N/A \\
\hline Appetite loss & $\begin{array}{c}5.87^{\mathrm{a}} \\
(4.45-7.30)\end{array}$ & $\begin{array}{c}4.08^{\mathrm{a}} \\
(2.90-5.26)\end{array}$ & $\begin{array}{c}3.81^{\mathrm{a}} \\
(2.25-5.37)\end{array}$ & $\begin{array}{c}3.90^{\mathrm{a}} \\
(1.78-6.03)\end{array}$ & N/A \\
\hline Constipation & $\begin{array}{c}6.98^{\mathrm{a}} \\
(5.38-8.59)\end{array}$ & $\begin{array}{c}7.07^{a} \\
(5.34-8.79)\end{array}$ & $\begin{array}{c}7.96^{a} \\
(5.29-10.63)\end{array}$ & $\begin{array}{c}5.29^{a} \\
(3.80-6.78)\end{array}$ & N/A \\
\hline Diarrhea & $\begin{array}{c}4.81^{\mathrm{a}} \\
(3.52-6.10)\end{array}$ & $\begin{array}{c}4.43^{\mathrm{a}} \\
(3.14-5.72)\end{array}$ & $\begin{array}{c}3.24^{\mathrm{a}} \\
(1.96-4.51)\end{array}$ & $\begin{array}{c}4.65^{\mathrm{a}} \\
(3.11-6.19)\end{array}$ & N/A \\
\hline Financial problems & $\begin{array}{c}5.23^{\mathrm{a}} \\
(3.77-6.69)\end{array}$ & $\begin{array}{c}8.22^{\mathrm{b}} \\
(6.16-10.28)\end{array}$ & $\begin{array}{c}12.30^{\mathrm{b}} \\
(8.41-16.19)\end{array}$ & $\begin{array}{c}7.71^{\mathrm{a}, \mathrm{b}} \\
(5.21-10.22)\end{array}$ & N/A \\
\hline
\end{tabular}

Table 2 continues on next page 
Continuation of table 1

\begin{tabular}{|c|c|c|c|c|c|}
\hline & \multicolumn{5}{|c|}{ Propensity weighted groups (mean, $\mathrm{Cl}$ ) } \\
\hline & BCS & MAS & A-BR & I-BR & GP \\
\hline Group size $(\mathrm{n})$ & 434.0 & 386.3 & 178.6 & 295.5 & N/A \\
\hline \multicolumn{6}{|l|}{ EORTC-QLQ-BR23 } \\
\hline \multicolumn{6}{|l|}{ Function scales: } \\
\hline Body image & $\begin{array}{c}87.45^{a} \\
(85.45-89.45)\end{array}$ & $\begin{array}{c}80.49^{b} \\
(78.24-82.74)\end{array}$ & $\begin{array}{c}82.28^{b} \\
(79.01-85.55)\end{array}$ & $\begin{array}{c}82.35^{\mathrm{b}} \\
(79.83-84.88)\end{array}$ & N/A \\
\hline Sexual functioning & $\begin{array}{c}27.90^{a} \\
(25.23-30.58)\end{array}$ & $\begin{array}{c}27.51^{\mathrm{a}} \\
(24.80-30.22)\end{array}$ & $\begin{array}{c}31.95^{\mathrm{a}, \mathrm{b}} \\
(28.02-35-89)\end{array}$ & $\begin{array}{c}33.35^{b} \\
(29.99-36.72)\end{array}$ & N/A \\
\hline Sexual enjoyment & $\begin{array}{c}57.03^{a} \\
(53.03-61.02)\end{array}$ & $\begin{array}{c}54.82^{\mathrm{a}} \\
(51.07-58.57)\end{array}$ & $\begin{array}{c}64.24^{b} \\
(59.27-69.21)\end{array}$ & $\begin{array}{c}63.80^{\mathrm{b}} \\
(60.06-67.54)\end{array}$ & N/A \\
\hline Future perspective & $\begin{array}{c}74.51^{\mathrm{a}} \\
(72.17-76.85)\end{array}$ & $\begin{array}{c}71.93^{\mathrm{a}} \\
(69.15-74.71)\end{array}$ & $\begin{array}{c}76.14^{\mathrm{a}} \\
(72.34-79.95)\end{array}$ & $\begin{array}{c}75.03^{\mathrm{a}} \\
(72.21-77.85)\end{array}$ & N/A \\
\hline \multicolumn{6}{|l|}{ Symptom scales: } \\
\hline Systemic therapy side-effects & $\begin{array}{c}12.60^{\mathrm{a}} \\
(11.57-13.64)\end{array}$ & $\begin{array}{c}12.74^{a} \\
(11.49-13.99)\end{array}$ & $\begin{array}{c}14.41^{\mathrm{a}} \\
(12.41-16.40)\end{array}$ & $\begin{array}{c}13.79^{a} \\
(12.08-15.50)\end{array}$ & N/A \\
\hline Breast symptoms & $\begin{array}{c}13.45^{\mathrm{a}} \\
(11.80-15.11)\end{array}$ & $\begin{array}{c}9.94^{b} \\
(8.60-11.28)\end{array}$ & $\begin{array}{c}8.79^{b} \\
(6.88-10.71)\end{array}$ & $\begin{array}{c}10.82^{\mathrm{a}, \mathrm{b}} \\
(8.36-13.27)\end{array}$ & N/A \\
\hline Arm symptoms & $\begin{array}{c}12.68^{\mathrm{a}} \\
(11.02-14.34)\end{array}$ & $\begin{array}{c}17.12^{\mathrm{b}} \\
(15.12-19.12)\end{array}$ & $\begin{array}{c}18.18^{b} \\
(14.94-21.41)\end{array}$ & $\begin{array}{c}16.82^{\mathrm{b}} \\
(13.60-20.03)\end{array}$ & N/A \\
\hline Hair loss & $\begin{array}{c}4.25^{\mathrm{a}} \\
(2.78-5.71)\end{array}$ & $\begin{array}{c}4.86^{a} \\
(3.37-6.35)\end{array}$ & $\begin{array}{c}6.50^{\mathrm{a}} \\
(4.02-8.98)\end{array}$ & $\begin{array}{c}5.47^{a} \\
(3.58-7.37)\end{array}$ & N/A \\
\hline \multicolumn{6}{|l|}{ BREAST-Q } \\
\hline Satisfaction with Breasts & $\begin{array}{c}65.52^{\mathrm{a}} \\
(63.43-67.61)\end{array}$ & $\begin{array}{c}60.65^{b} \\
(58.79-62.51)\end{array}$ & $\begin{array}{c}71.29^{c} \\
(68.66-73.92)\end{array}$ & $\begin{array}{c}59.39^{b} \\
(57.18-61.60)\end{array}$ & N/A \\
\hline Satisfaction with Outcome & N/A & $\mathrm{N} / \mathrm{A}$ & $\begin{array}{c}75.75^{a} \\
(72.52-78.99)\end{array}$ & $\begin{array}{c}66.37^{\mathrm{b}} \\
(63.66-69.08)\end{array}$ & N/A \\
\hline Psychosocial Well-being & $\begin{array}{c}73.77^{\mathrm{a}} \\
(71.70-75.83)\end{array}$ & $\begin{array}{c}66.50^{\mathrm{b}} \\
(64.68-68.32)\end{array}$ & $\begin{array}{c}75.78^{\mathrm{a}, \mathrm{c}} \\
(72.94-78.63)\end{array}$ & $\begin{array}{c}71.60^{\mathrm{a}} \\
(69.30-73.90)\end{array}$ & N/A \\
\hline Sexual Well-being & $\begin{array}{c}62.70^{\mathrm{a}} \\
(59.92-65.48)\end{array}$ & $\begin{array}{c}50.00^{\mathrm{b}} \\
(47.44-52.55)\end{array}$ & $\begin{array}{c}63.33^{\mathrm{a}} \\
(58.91-67.75)\end{array}$ & $\begin{array}{c}56.38^{c} \\
(52.88-59.88)\end{array}$ & N/A \\
\hline Physical Well-being: Chest & $\begin{array}{c}67.39^{a} \\
(65.17-69.61)\end{array}$ & $\begin{array}{c}73.47^{\mathrm{b}} \\
(71.78-75.16)\end{array}$ & $\begin{array}{c}75.81^{b, c} \\
(73.56-78.06)\end{array}$ & $\begin{array}{c}72.64^{b} \\
(70.61-74.66)\end{array}$ & N/A \\
\hline Physical Well-being: Abdomen & N/A & N/A & $\begin{array}{c}75.81 \\
(73.56-78.06)\end{array}$ & $\mathrm{N} / \mathrm{A}$ & N/A \\
\hline Satisfaction Nipple & & & $\begin{array}{c}63.03^{a} \\
(58.82-67.25)\end{array}$ & $\begin{array}{c}54.96^{\mathrm{b}} \\
(49.38-60.54)\end{array}$ & N/A \\
\hline
\end{tabular}

BCS: breast conserving surgery, MAS: mastectomy without breast reconstruction, A-BR: mastectomy with autologous breast reconstruction, I-BR: mastectomy with implant breast reconstruction, GP: general population. VAS: Visual Analogue Scale, CI: $95 \%$ Confidence interval.

Values in the same row and subtable not sharing the same subscript $(a, b, c)$ were significantly different at $p<0.05$ using the adjusted Wald test. Cells with no subscript were not included in the test. Tests assume equal variances. 


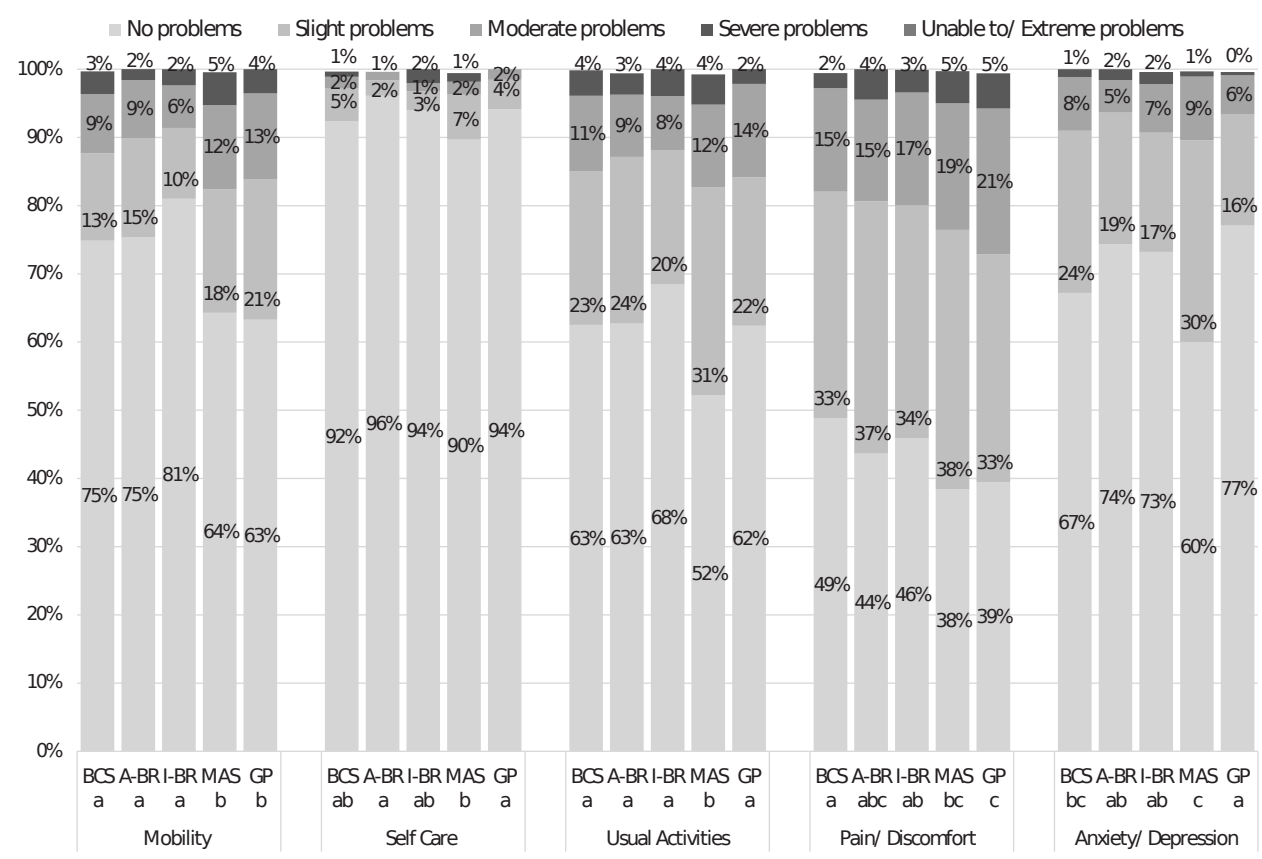

Figure 1: Propensity-weighted EQ-5D-5L sub-scale per surgical treatment and weighted Dutch general population (GP)

$B C S$ : breast conserving surgery, MAS: mastectomy without breast reconstruction, A-BR: mastectomy with autologous breast reconstruction, I-BR: mastectomy with implant breast reconstruction, GP: general population.

Values in the same domain not sharing the same subscript were significantly different at $p<0.05$ using the adjusted Wald test. Cells without subscript were not included in the test. Tests assume equal variances.

Table 3: Mean EQ-5D-5L utilities for 1871 breast cancer patients with or without surgical complications per treatment group and the Dutch general population after propensity-weighted adjustment

\begin{tabular}{lccccc}
\hline \multicolumn{5}{c}{ Adjusted group } \\
\hline Group size (n) & BCS & MAS & A-BR & I-BR & GP \\
\hline No complications & 434.0 & 386.3 & 178.6 & 295.5 & N/A \\
\hline $\begin{array}{l}\text { Mean EQ-5D-5L utilities } \\
\text { (Cl) }\end{array}$ & $82.3 \%$ & $75.0 \%$ & $53.4 \%$ & $68.4 \%$ & $100 \%$ \\
\hline Complications & $0.859^{\mathrm{a}}$ & $0.818^{\mathrm{b}}$ & $0.878^{\mathrm{a}}$ & $0.847^{\mathrm{a}, \mathrm{b}}$ & $0.833^{\mathrm{b}}$ \\
\hline $\begin{array}{l}\text { Mean EQ-5D-5L utilities } \\
\text { (Cl) }\end{array}$ & $10.844-0.875)$ & $(0.796-0.840)$ & $(0.854-0.902)$ & $(0.810-0.884)$ & $(0.812-0.854)$ \\
\hline
\end{tabular}

$\mathrm{BCS}$ : breast conserving surgery, MAS: mastectomy without breast reconstruction, BR: breast reconstruction, A-BR: mastectomy with autologous BR, I-BR: mastectomy with implant BR, GP: general population. Cl: $95 \%$ Confidence interval.

Values in the same row and subtable not sharing the same subscript $(a, b)$ were significantly different at $p<0.05$ using the adjusted Wald test. Cells with no subscript were not included in the test. Tests assume equal variances. 


\section{Effect of complications on EQ-5D outcomes}

A total of $96 / 615$ (16\%) of BCS, 112/507 (22\%) of MAS, 138/330 (42\%) of A-BR, and 140/419 (33\%) of I-BR-patients reported to have experienced complications following surgery (unadjusted groups). After propensity-weighted adjustment, patients treated with either BCS, MAS, or A-BR who had experienced complications, showed statistically significant lower mean utilities than patients from the same groups who had not experienced complications (Table 3). However, MAS patients without complications $(0.818$ reported means similar to A-BR-patients with complications (0.816) and I-BR-patients with complications (0.861). Mean EQ-5D scores of MAS-patients who previously had undergone a BR (e.g. failed BR) and who never had undergone a BR did not differ significantly from each other ( $p=0.943$, results not shown).

For both BR groups, problems reported per EQ-5D domain were stratified by complications (Figure 2). A-BR patients with complications reported statistically significant more often problems for the 'Usual activities' and "Pain/Discomfort" domain than A-BR patients without complications.

Figure 3 includes boxplots presenting timelines of the utilities for A-BR and I-BR patients who had experienced complications, adjusted by propensity weighting. In the first three years after a complicated $B R$, a relatively large proportion of $A-B R$ patients experienced a severe impact on HRQoL. This negative impact on HRQoL recovered with time for both treatment modalities. However, a larger proportion of I-BR patients seemed to recover up to the degree that they did not report problems on any of the EQ-5D dimensions within 6-9 years after the last BR surgery, compared to a much smaller portion of A-BR patients.

\section{Discussion}

This study aimed to compare HRQoL outcomes for four common surgical breast cancer treatment options (BSC, MAS, A-BR, I-BR) to improve the evidence and consequently decision-making about breast cancer surgery. We found that MAS-patients reported the lowest mean HRQoL (EQ-5D) and functioning (EORTC-QLQ-C30). Based on the Breast-Q, A-BR-patients had statistically significant higher 'satisfaction with outcome' and 'satisfaction with breasts' and 'sexual well-being' scores than I-BR patients. Patients with complications (except for I-BR) reported statistically significant lower HRQoL (EQ-5D) than patients without complications; complications in A-BR patients resulted in a substantially lower HRQoL than in I-BR patients.

The results show the added value of breast conservation and reconstruction compared to MAS, however, the differences between BCS, I-BR, and A-BR were subtle. Indeed, we found many statistically significant differences, but given the high statistical power of our large study most of them were small. So, on average we found few clinically relevant differences between BCS, $\mathrm{I}-\mathrm{BR}$, and A-BR for the various HRQoL domains. However, the benefits of these subtle differences over a long time are a good reason to consider them in clinical decision-making, specifically when considering the effects of complications and the patients' attitude towards the risks of complications. 


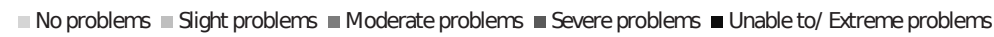
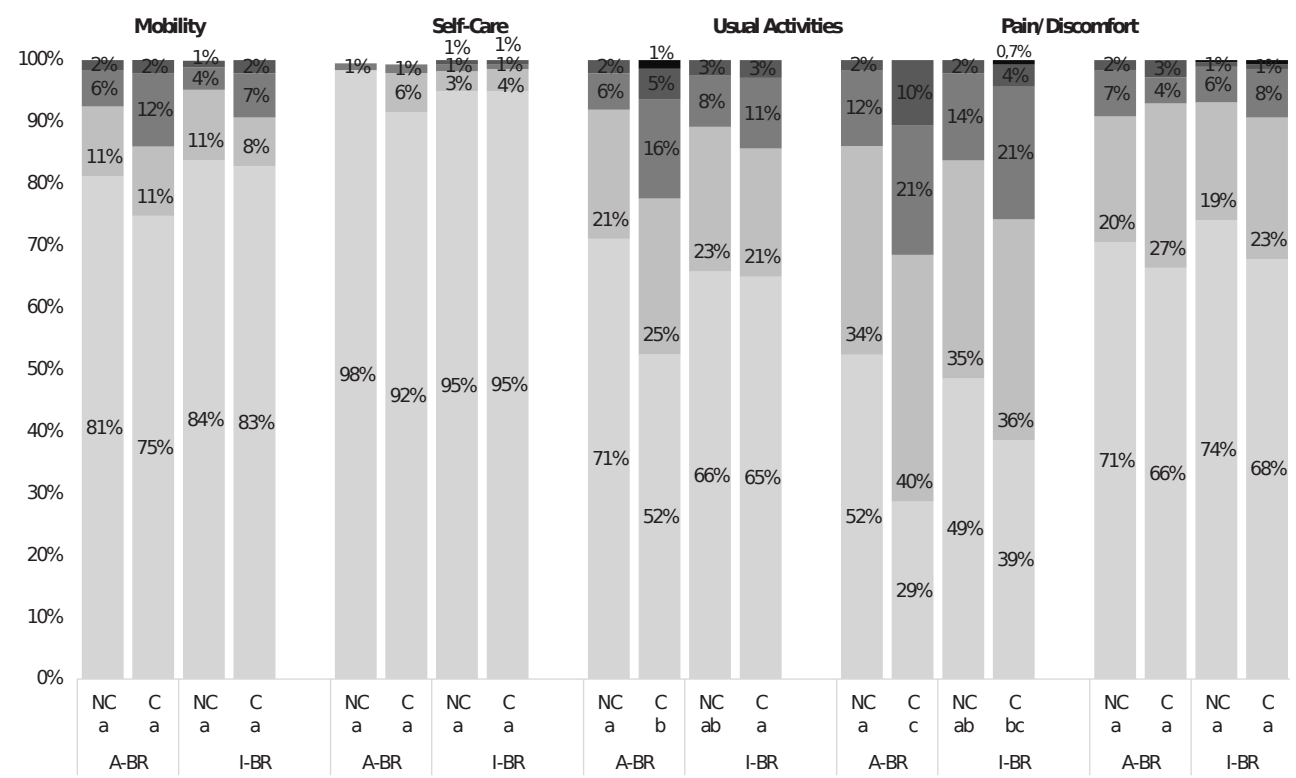

Figure 2: EQ-5D-5L sub-scale contrasting A-BR and I-BR with or without surgical complication (unadjusted for propensity score)

A-BR: mastectomy with autologous BR, I-BR: mastectomy with implant BR, GP: general population. $\mathrm{C}=$ surgical complications; $\mathrm{NC}=$ no surgical complications.

Values in the same domain not sharing the same subscript were significantly different at $p<0.05$ using the Adjusted Wald test. Cells without subscript were not included in the test. Tests assume equal variances.

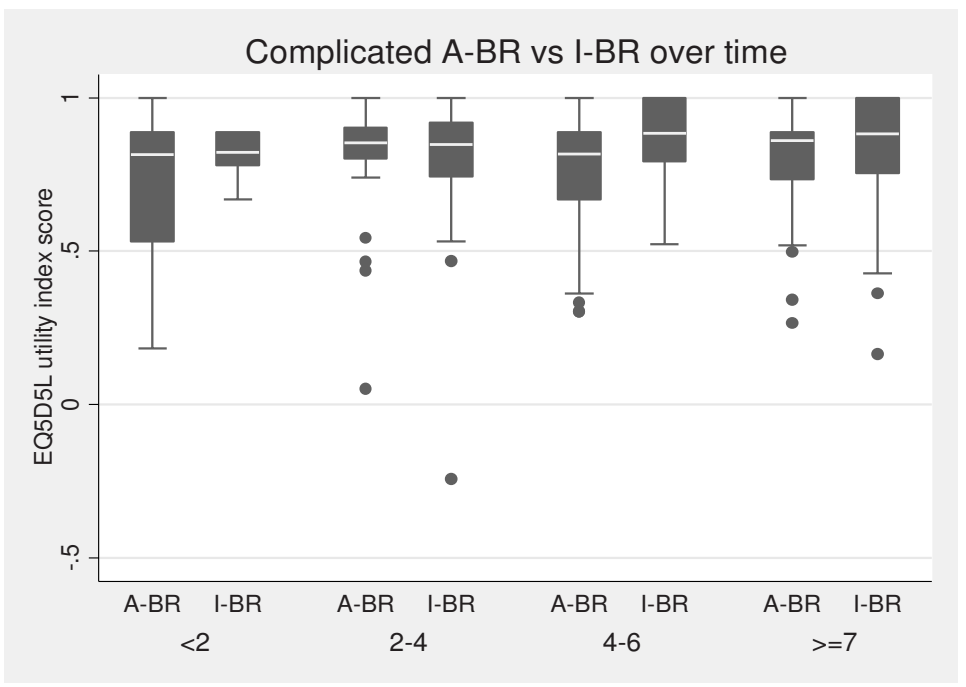

Figure 3: EQ-5D-5L utilities over time in years (time between last BR and questionnaire) for breast cancer patients following breast reconstruction (BR) with complications, after propensity-weighted adjustment A-BR: mastectomy with autologous BR, I-BR: mastectomy with implant BR 
Only one other study that reported utilities for surgically-treated breast cancer patients receiving BR was found (Immediate IBR-patients, mean score:0.83) [14]. We found lower mean HRQoL scores reported by MAS-patients and benefits in some HRQoL domains for BCS-patients (higher 'Body Image' and more favorable 'Arm Symptom') over all other surgically treated groups, which confirms previously reported results [15, 20,32]. The benefits of A-BR compared to I-BR (higher mean 'Satisfaction with Breasts/Outcome/Nipple', and 'Sexual Well-being') were also reported by Santosa et al. [22]. However, in contrast, both the present study and Thorarinsson et al. [33] did not find statistically significant differences between A-BR and I-BR on either EQ-5D-5L or EORTCQLQ outcomes. So, although the BREAST-Q results suggest that the patients' perception of their reconstructed breast(s) is favorable for A-BR over I-BR, this does not necessarily lead to better outcomes in terms of overall HRQoL.

The second aim was to assess the impact of complications after different surgical treatments. Indeed, if any clinically relevant differences were found [34], they seemed to be related to complications. Specifically for A-BR, one should not ignore the impact of complications [35]. A-BR patients with complications (versus those without complications) had statistically significant lower mean utilities as measured with the EQ-5D, and more often had problems in the 'Usual activities' and 'Pain/Discomfort' domains. Also, mean scores recovered faster for I-BR patients than A-BR patients. Finally, a larger proportion of A-BR patients never recovered up to the degree that they did not report problems on any of the EQ-5D dimensions.

The faster recovery after complications of I-BR patients could explain why the utilities in patients with and without complications did not differ statistically. The symptoms and the longer lasting impact of complications in A-BR patients may be inherent to the type of complications associated with these procedures. More specifically, failure of an I-BR is often due to an infection, resulting in removal of the implant, later often followed by a new I-BR. Total flap failure following A-BR requires a new and additional donor-site, with its own donor-site issues and complication risks. Women experience BR (flap) failure as an emotionally very difficult life event [36], although previous studies have shown that physical and mental health after a BR complication generally recover to normal levels after a period of time $[37,38]$.

By measuring $\mathrm{HRQ}$ oL using multiple, validated PROMs in a large sample of patients following different types of breast cancer surgery, we were able to improve earlier, smaller and less consistent attempts to assess HRQoL in surgically-treated breast cancer patients. Our statistically significant results confirm the findings of studies mentioned previously $[15,20,22,32,35]$, thereby supporting the added value of breast conservation and reconstruction for breast cancer patients.

Furthermore, no predominant treatment option was found. This stresses the idea that all treatment options (which are physically feasible) should be considered for every patient. The ultimate treatment decision should be predominantly based on the patient's preferences, resulting in the alignment of the favorable assets (or domain scores) of each procedure and the patient's goals and expectations with the expected final result of each procedure as well as their attitudes towards complication risks. Although we have investigated decision-making in a previous study in 
a similar cohort of patients [39], it would have been interesting to have insight in the treatment rationale for the current patient cohort.

This study demonstrates the utilization of the unique assets of the EQ-5D, a 'preference-based', standardized generic measure of health status which is suitable for a wide comparison of treatment options [40,41]. A benefit of this preference-based HRQoL measure compared to commonly applied 'non-preference-based' measures like the EORTC and Breast- $Q$ is that its outcomes can be aggregated over time and, after multiplication with survival time, provides Quality Adjusted Life Years (QALYs) [41]. The EQ-5D utilities can be related to the time period of each health state, and can therefore combine the 'utility' of the advantages and disadvantages, such as complications of surgical procedures. Note that our data were not able to fully solve the question how the utility of the benefits of a surgical procedure relates to the disutility of complications, as this requires longitudinal data to represent the EQ-5D values and the time lived with or without a given complication. Nevertheless, the present data can still provide insights in the trade-off between benefits and complications of the different surgical procedures.

Of further importance is that previous studies have not yet described 'utilities' for the complete range of breast cancer surgery options. This currently complicates the implementation of health economics and reimbursement decision-making. Clinical treatment value should be related to healthcare costs, which is the ultimate goal towards creating value-based health care [42]. In our subsequent study, we will relate costs to the outcomes we found in the present study.

Some limitations are relevant in the interpretation of our results. First, although propensityweighted adjustment was employed to minimize the effects of bias caused by including patients from an observational cohort, one cannot exclude that relevant variables may still have influenced the results of our study [43]. For instance, surgical treatment selection might be based on severity of comorbidities or performance status, which were both not available in our data. Non-response bias could have been induced by socioeconomic and procedure-related differences, as described by Berlin et al. [44] Besides, surveys introduce a certain arbitrariness, as patients might understand or interpret questions or experiences other than intended.

We conclude that HRQoL of MAS-patients was often the lowest, supporting the added value of breast conservation and reconstruction in breast cancer patients. Furthermore, we found that each surgical procedure has subtle favorable assets, the most notable related to complications: a complication in A-BR patients resulted in a substantially lower HRQoL than in I-BR patients and MAS-patients without complications had similar or lower mean EQ-5D scores to A-BR or I-BR patients with complications. This could support a discussion about the alignment of the patient's goals, expectations and attitudes towards complication risks with the expected final result of each procedure. 


\section{References}

1. Janssen-Heijnen MLG, van Steenbergen LN, Voogd AC, Tjan-Heijnen VCG, Nijhuis PH, Poortmans PM, et al. Small but significant excess mortality compared with the general population for long-term survivors of breast cancer in the Netherlands. Annals of Oncology. 2014;25:64-8.

2. Howlader N, Noone AM, Krapcho M, Miller D, Bishop K, Kosary CL, et al. SEER Cancer Statistics Review, 1975-2014 Bethesda, MD: National Cancer Institute; 2017 [updated Based on November 2016 SEER data submission, posted to the SEER web site April 2017. Available from: https://seer.cancer.gov/csr/1975_2014/.

3. van Maaren MC, de Munck L, de Bock GH, Jobsen JJ, van Dalen T, Linn SC, et al. 10 year survival after breastconserving surgery plus radiotherapy compared with mastectomy in early breast cancer in the Netherlands: a population-based study. Lancet Oncol. 2016;17(8):1158-70.

4. Fischer B, Andersen S, Bryant J, Margolese RG, Deutsch M, ER F, et al. Twenty-year follow-up of a randomized trial comparing total mastectomy, lumpectomy, and lumpectomy plus irradiation for the treatment of invasive breast cancer. N Engl J Med. 2002;347(16):1233-41.

5. Litière $S$, Werutsky G, Fentiman IS, Rutgers $E$, Christiaens MR, Van Limbergen $E$, et al. Breast conserving therapy versus mastectomy for stage I-II breast cancer: 20 year follow-up of the EORTC 10801 phase 3 randomised trial. Lancet Oncol. 2012;13(4):412-9.

6. Cordeiro PG. Breast Reconstruction after Surgery for Breast Cancer. New England Journal of Medicine. 2008(359):1590-601.

7. Netherlands Comprehensive Cancer Organisation (IKNL). National guideline on Breast Cancer. Netherlands Comprehensive Cancer Organisation (IKNL),,; 2012.

8. Senkus E, Kyriakides S, Ohno S, Penault-Llorca F, Poortmans P, Rutgers E, et al. Primary breast cancer: ESMO Clinical Practice Guidelines for diagnosis, treatment and follow-up. Annals of Oncology. 2015;26:8-30.

9. Mureau MAM, Nederlandse Vereniging voor Plastische Chirurgie (NVPC). Dutch Breast Reconstruction Guideline. Journal of Plastic Reconstructive and Aesthetic Surgery 2018:290-304.

10. Tondu T, Tjalma WAA, Thiessen FEF. Breast reconstruction after mastectomy. European Journal of Obstetrics \& Gynecology and Reproductive Biology. 2018;article in press.

11. Damen TH, Wei W, Mureau MAM, Tjong-Joe-Wai R, Hofer SO, Essink-Bot ML, et al. Medium-term cost analysis of breast reconstructions in a single Dutch centre: a comparison of implants, implants preceded by tissue expansion, LD transpositions and DIEP flaps. J Plast Reconstr Aesthet Surg. 2011;64(8):1043-53.

12. Bennett KG, Qi J, Kim HM, Hamill JB, Pusic AL, Wilkins EG. Comparison of 2-Year Complication Rates Among Common Techniques for Postmastectomy Breast Reconstruction. JAMA surgery. 2018.

13. Pinsolle V, Grinfeder C, Mathoulin-Pelissier S, Faucher A. Complications analysis of 266 immediate breast reconstructions. J Plast Reconstr Aes. 2006;59:1017-24.

14. Robertson S, Wengstrom Y, Eriksen C, Sandelin K. Breast surgeons performing immediate breast reconstruction with implants - assessment of resource-use and patient-reported outcome measures. Breast (Edinburgh, Scotland). 2012;21(4):590-6

15. Jeevan R, Cromwell DA, Browne JP, Caddy CM, Pereira J, Sheppard C, et al. Findings of a national comparative audit of mastectomy and breast reconstruction surgery in England. J Plast Reconstr Aes. 2014;67(10):133344.

16. Mols F, Vingerhoets AJJM, Coebergh JW, van de Poll-Franse LV. Quality of life among long-term breast cancer survivors: A systematic review. European Journal of Cancer. 2005;41:2613-9.

17. Ganz PA, Desmond KA, Leedham B, Rowland JH, Meyerowitz BE, Belin TR. Quality of Life in Long-Term, Disease-Free Survivors of Breast Cancer: a Follow-up Study. J Natl Cancer Inst. 2002;94:39-49.

18. Tan ML, Idris BD, Teo LW, Loh SY, Seow GC, Chia YY, et al. Validation of EORTC QLQ-C30 and QLQ-BR23 questionnaires in the measurement of quality of life of breast cancer patients in Singapore. Asia-Pacifi $C$ Journal of Oncology Nursing. 2014;1(1).

19. Schmidt ME, Wiskemann J, Steindorf K. Quality of life, problems, and needs of disease-free breast cancer survivors 5 years after diagnosis. Quality of Life Research. 2018;27:2077-86.

20. Sun Y, Kim S-W, Heo CY, Kim D, Hwang Y, Yom CK, et al. Comparison of Quality of Life Based on Surgical Technique in Patients with Breast Cancer. Jpn J Clin Oncol. 2014;44(1):22-7. 
21. Lee C, Sunu C, Pignone M. Patient-Reported Outcomes of Breast Reconstruction after Mastectomy: A Systematic Review. J Am Coll Surgeons. 2009;209(1):123-33.

22. Santosa KB, Qi J, Kim HM, Hamill JB, Wilkins EG, Pusic AL. Long-term Patient-Reported Outcomes in Postmastectomy Breast Reconstruction. JAMA surgery. 2018;153(10):891-9.

23. Versteegh MM, Vermeulen KM, Evers SMAA, de Wit GA PR, Stolk EA. Dutch Tariff for the Five-Level Version of EQ-5D. Value Health. 2016;19:343-52.

24. Aaronson NK, Ahmedzai S, Bergman B, Bullinger M, Cull A, Duez NJ, et al. The European Organisation for Research and Treatment of Cancer QLQ-C30: A quality-of-life instrument for use in international clinical trials in oncology. J Natl Cancer Inst. 1993;85:365-76.

25. Sprangers MAG, Groenvold M, Arraras JI, Franklin J, te Velde A, Muller M, et al. The European Organisation for Research and Treatment of Cancer: Breast Cancer Specific Quality of Life Questionnaire Module: First results from a three-country field study. J Clin Oncol. 1996;14:2756-68.

26. Fayers PM, Aaronson NK, Bjordal K, Groenvold M, Curran D, Bottomley A, et al. The EORTC QLQ-C30 Scoring Manual (3rd Edition). Brussels: European Organisation for Research and Treatment of Cancer; 2001.

27. Cano SJ, Klassen AF, Scott AM, Pusic AL. A Closer Look at the BREAST-Q. Clin Plastic Surg. 2013;40:287-96.

28. McCaffrey DF, Griffin BA, Almirall D SM, Ramchand R, Burgette LF. A Tutorial on Propensity Score Estimation for Multiple Treatments Using Generalized Boosted Models. Stat Med. 2013;32(19):3388-414.

29. Griffin BA, Ridgeway G, Morral AR, Burgette LF, Martin C, Almirall D, et al. Toolkit for Weighting and Analysis of Nonequivalent Groups (TWANG). Santa Monica, CA: RAND Corporation; 2014.

30. StataCorp L. STATA SURVEY DATA REFERENCE MANUAL.

31. StataCorp. Stata Statistical Software: Release 14. In: College Station TSL, , editor. 2015.

32. Eltahir Y, Werners LLCH, Dreise MM, IA ZvE, Jansen L, Werker PMN, et al. Quality-of-life outcomes between mastectomy alone and breast reconstruction: comparison of patient-reported BREAST-Q and other healthrelated quality-of-life measures. Plastic and reconstructive surgery. 2013;132(2):201e-9e.

33. Thorarinsson A, Fröjd V, Kölby L, Ljungdal J, Charles Taft C, Mark H. Long-Term Health-Related Quality of Life after Breast Reconstruction: Comparing 4 Different Methods of Reconstruction. Plast Reconstr Surg Glob Open. 2017;5:e1316.

34. Cocks K, King MT, Velikova G, St-James MM, Fayers PM, Brown JM. Evidence-Based Guidelines for Determination of Sample Size and Interpretation of the European Organisation for the Research and Treatment of Cancer Quality of Life Questionnaire Core 30. Journal of Clinical Oncology. 2011;29(1):89-96.

35. Gopie JP, Timman R, Hilhorst MT, Hofer SOP, Mureau MAM, Tibben A. The short-term psychological impact of complications after breast reconstruction. Psychooncology. 2013;22:290-8.

36. Higgins KS, Gillis J, Williams JG, LeBlanc M, Bezuhly M, Chorney JM. Women's Experiences With Flap Failure After Autologous Breast Reconstruction: A Qualitative Analysis. Ann Plas Surg. 2017;78(5):521-5.

37. Timman R, Gopie JP, Brinkman JN, Kleijne A, Seynaeve C, Menke-Pluymers MBE, et al. Most women recover from psychological distress after postoperative complications following implant or DIEP flap breast reconstruction: A prospective long-term follow-up study. PloS one. 2017;12(3):e0174455.

38. Lu SM, Nelson JA, Fischer JP, Fosnot J, Goldstein J, Selber JC, et al. The impact of complications on function, health, and satisfaction following abdominally based autologous breast reconstruction: A prospective evaluation. J Plast Reconstr Aes 2014;67(5):682-92.

39. de Ligt KM, van Bommel ACM, Schreuder K, Maduro JH, Vrancken Peeters MTFD, Mureau MAM, et al. The effect of being informed on receiving immediate breast reconstruction in breast cancer patients. European journal of surgical oncology : the journal of the European Society of Surgical Oncology and the British Association of Surgical Oncology. 2018;44(5):717-24.

40. Herdman M, Gudex C, Lloyd A, Janssen MF, Kind P, Parkin D, et al. Development and preliminary testing of the new five-level version of EQ-5D (EQ-5D-5L) Qual Life Res. 2011;20:1727-36.

41. Gray AM, Clarke PM, Wolstenholme JL, Wordsworth S. Chapter 5: Measuring, valuing, and analysing health outcomes. Applied Methods of Cost-effectiveness Analysis in Health Care. Handbooks in Health Economic Evaluation Series. Oxford: Oxford University Press; 2011.

42. Porter ME, Teisberg EO. How Physicians Can Change the Future of Health Care. JAMA. 2007;297(10):110311. 
43. McCaffrey DF, Griffin BA, Almirall D, Slaughter ME, Ramchand R, Burgette LF. A Tutorial on Propensity Score Estimation for Multiple Treatments Using Generalized Boosted Models. Stat Med. 2013;32(19):3388-414.

44. Berlin NL, Hamill JB, Qi J, Kim HM, Pusic AL, Wilkins EG. Nonresponse bias in survey research: lessons from a prospective study of breast reconstruction. Journal of Surgical Research. 2018;224:112-20. 



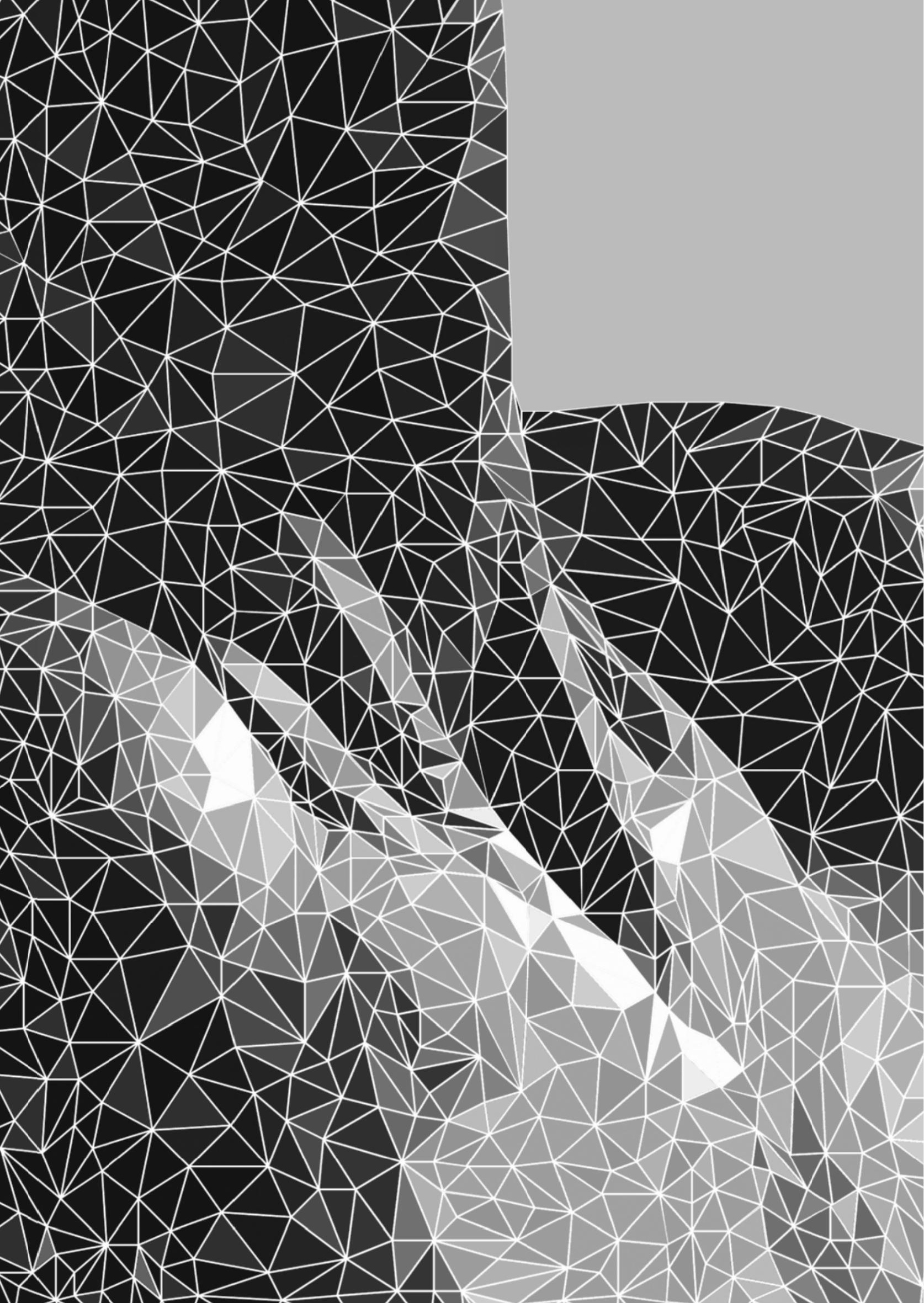


CHAPTER 4

THE EFFECT OF BEING INFORMED ON RECEIVING IMMEDIATE BREAST RECONSTRUCTION IN BREAST CANCER PATIENTS

K.M. de Ligt

A.C.M. van Bommel

K. Schreuder

J.H. Maduro

M.T.F.D. Vrancken Peeters

M.A.M. Mureau

S. Siesling

- On behalf of the NABON Breast Cancer Audit Working Group -

Eur J Surg Oncol. 2018 May;44(5):717-724. 



\begin{abstract}
Introduction:

In previous research from the NABON breast cancer audit, observed hospital variation in immediate breast reconstruction (IBR) rates in the Netherlands could not be fully explained by tumour, patient, and hospital factors. The process of information provision and decision-making may also contribute to the observed variation; the objective of the current study was to give insight in the underlying decision-making process for IBR and to determine the effect of being informed about IBR on receiving IBR.
\end{abstract}

\title{
Methods:
}

A total of 502 patients with IBR and 716 without IBR treated at twenty-nine hospitals were invited to complete an online questionnaire on obtained information and decision-making regarding IBR. The effect of being informed about IBR on receiving IBR was determined by logistic regression analysis.

\section{Results:}

Responses from five hundred and ten patients ( $n=229$ IBR, $n=281$ without IBR) were analysed. Patients with IBR compared to patients without reconstruction showed a difference in patient, tumour, treatment (including radiotherapy), and hospital characteristics. Patients with IBR were more often informed about IBR as a treatment option (99\% vs 73\%), they discussed (dis)advantages more often with their physician ( $86 \%$ vs $68 \%$ ), and they were more often involved in shared decision-making ( $91 \%$ vs $67 \%$ ) compared to patients without IBR. Multivariate logistic regression analysis, corrected for confounders, showed that being informed about IBR increased the odds for receiving IBR fourteen times $(p<0.001)$.

\section{Conclusions:}

The positive effect of being informed about IBR on receiving IBR stresses the importance of treatment information in the decision-making process for IBR. 


\section{Introduction}

In 2014, about 14,500 women were diagnosed with invasive breast cancer and 2,300 with Ductal Carcinoma in Situ (DCIS) in the Netherlands [1]. Surgical procedures as mastectomy and breast conserving therapy combined with adjuvant radiotherapy have been shown to offer equivalent survival $[2,3]$. However, loss of one or both breasts mutilates the female appearance and consequently, mastectomy may negatively impact body image and sexuality, leading to feelings of anxiety and depression [4, 5]. These effects may be minimized by restoring the contour of the breast with a breast reconstruction [6-8]. Breast reconstruction may be performed either directly after mastectomy in the same operation, which is known as immediate breast reconstruction (IBR), or in a separate operation, some time after the mastectomy, which is called a delayed breast reconstruction (DBR) [9]. IBR can be safely performed without affecting patient survival $[10,11]$ or hampering detection of local recurrences [10-12].

Although in the Netherlands the national guideline on breast cancer treatment recommends considering IBR for every patient needing mastectomy [3], the average IBR-rate of about $20 \%$ was rather low in 2014 [13], albeit comparable to other countries [14]. In the NABON ('National Breast Cancer Consultation the Netherlands') Breast Cancer Audit (NBCA)[15] we previously demonstrated varying IBR-rates between Dutch hospitals from 0-83\% (DCIS) and 0-64\% (invasive breast cancer), which could not be fully explained by tumour, patient, and hospital factors $[13,16]$. However, it could well be that other reasons for this observed variation exist, such as preoperative information provision about IBR, shared decision-making (SDM), and patient or physician preferences.

Aside from the recommendation to consider IBR in every mastectomy patient, the guideline also recommends physicians to provide sufficient and timely information to patients [3]. In the Netherlands, IBR is performed by plastic surgeons; therefore, consultation between surgeon and plastic surgeon and referral of patients for a consultation with a plastic surgeon is recommended [17]. Although information facilitates SDM [18], current information provision about IBR may be insufficient [19-21]. Considering the positive effects of both IBR and SDM (for instance about treatment decisions) on the quality of life [7, 22-24], psychosocial functioning [25-28], and patient satisfaction [29-31] of mastectomy patients, breast reconstruction should ideally be performed whenever feasible [22] and more importantly, preoperatively discussed with patients in a process of SDM. Therefore, the objective of the present study was to investigate the underlying decisionmaking processes patients experienced during the preoperative consultations for their breast cancer surgery with or without IBR in the Netherlands. The second aim was to determine the effect of being informed about IBR on actually receiving IBR.

\section{Methods}

\section{Study population}

Twenty-nine hospitals ( $1 / 3$ rd of the total number of hospitals in the Netherlands) volunteered to participate in the study. These hospitals were general $(n=15)$, teaching $(n=10)$, academic 
hospitals ( $n=4$, including a cancer-specific hospital), and all offered IBR in-house or referring to IBR-performing hospitals based on the national guideline as mentioned in the introduction. All patients that fit the inclusion criteria (female, aged $\geq 18$ years, diagnosed with DCIS or invasive breast cancer, treated with mastectomy between January 2013 and October 2014, no distant metastases) were selected from the Netherlands Cancer Registry (NCR), a national registry in which all newly diagnosed cancer patients are registered annually. Based on our power calculation (Appendix 1), fifty patients ( 25 with IBR and 25 without IBR) were randomly selected from every participating hospital by assigning them a random value between 0-1 and including those with the lowest values. In consultation with each hospital, we then excluded patients with recent recurrent disease (we did not want to bother patients currently receiving treatment) and patients who were unfit to fill in a questionnaire (due to psychological difficulties (dementia, depression) or language limitations). Since information about DBR is not collected in the NCR, we could not exclude patients with DBR on beforehand. The survey was hosted in PROFILES ('Patient Reported Outcomes Following Initial treatment and Long term Evaluation of Survivorship'), an online secured environment which facilitates data collection on patient-reported outcome measures (PROMs) from cancer survivors [32]. Paper questionnaires were provided on request. Invitations were sent out to selected patients from January 8th to May 29th 2015; responses were collected until July 30th, 2015. Respondents gave consent for processing their completed questionnaires and to merge them with the clinical data available in the NCR and NBCA. According to the Central Committee on Research involving Human Subjects (CCMO), this type of study does not require approval from an ethics committee in the Netherlands. This study was approved by the Privacy Review Board of the NCR.

\section{Questionnaire}

The questionnaire was specifically developed for this study and included items on patient characteristics, general health, breast cancer treatment, and breast reconstruction (Appendix 2). In addition, questions about SDM were categorized according to the definition of SDM: acknowledging a decision is required by knowing that IBR is an option, understanding and weighing all available information about the treatment options, and incorporating the patients' preferences in the final decision [18]. The questionnaire was tested for readability and comprehensibility by a panel of former breast cancer patients (members of the Dutch Breast Cancer Patient Association, 'Borstkankervereniging Nederland') before deployment.

\section{Analysis}

Patients who reported they had had DBR were excluded. Statistical analyses were performed in three steps. First, characteristics of respondents with IBR versus without IBR were compared using Pearson Chi-square tests. Second, patient responses regarding information provision and decisionmaking items were described and compared using Chi-square tests. In the third step, a multivariate logistic regression analysis was performed to determine the effect of being informed about IBR on receiving IBR, controlled for patient, tumour, and treatment characteristics that appeared to have a statistically significant relation with IBR in univariate analyses (relaxed significance level $p<0.10$ ); the significance level within the multivariate analyses was $p<0.05$. The following variables were include in the univariate analysis: age, body mass index (BMI), number of comorbidities, highest 
completed education, stage of disease (clinical), multifocality, unilateral or bilateral mastectomy, axillary dissection, neoadjuvant chemotherapy, radiotherapy, and IBR hospital volume. Variables were selected based on our previous research $[13,17]$ and literature on factors affecting the use of IBR [32].

All statistical analyses were performed using STATA (STATA Version 14) [33].

\section{Results}

\section{Respondents}

Five hundred and two patients with IBR and 716 without IBR received an invitation. Two hundred and fifty-three patients who had received IBR and 305 patients without IBR responded, giving a total of 558 responses (46\%). Twenty-four patients were excluded due to incomplete questionnaires, leading to valid data from 534 patients ( $n=229$ IBR, $n=305$ without IBR). Twenty-four patients who reported they had had DBR were excluded for the analyses, leaving 281 patients without IBR. No statistically significant differences between respondents and non-respondent groups were found in baseline characteristics (tumour morphology, year of surgery, IBR hospital volume) other than that respondents were younger than non-respondents $(p<0.001)$. The respondent group consisted of relatively more patients who had received IBR compared to the non-respondent group $(p=0.027$; data not shown). Respondents with IBR compared to respondents without IBR significantly differed in patient (age, education, socioeconomic status, comorbidities, BMI), tumour (stage, grade, lymph node status, multifocality), and treatment characteristics (unilateral or bilateral (prophylactic) mastectomy, radiotherapy), as well as hospital factors (IBR hospital volume, hospital type; all $p$-values< 0.05 ). Both groups were equally treated with chemotherapy ( $46 \%$ vs $51 \%, p=0.301$ ) and equally received neoadjuvant treatment ( $11 \%$ vs $17 \%$, borderline significance: $p=0.085$; Table 1 ).

The majority of patients with IBR either had received a tissue expander followed by a definite implant $(55 \%)$ or a direct-to-implant $(32 \%)$ reconstruction; other reconstruction types were latissimus dorsi flap (4\%), DIEP flap (5\%), or Superior Gluteal Artery Perforator (SGAP) flap (1\%; 3\% unknown; data not shown in Table 1).

Table 1: patient, tumour, treatment, and hospital characteristics

\begin{tabular}{|c|c|c|c|c|c|}
\hline & $\begin{array}{c}\text { IBR } \\
(n=229)\end{array}$ & $\%$ & $\begin{array}{c}\text { No IBR } \\
(n=281)\end{array}$ & $\%$ & p-value* \\
\hline \multicolumn{6}{|l|}{ Patient characteristics } \\
\hline \multicolumn{6}{|l|}{ Age in years (at diagnosis) } \\
\hline$<40$ & 31 & $14 \%$ & 9 & $3 \%$ & \multirow{3}{*}{$<0.001$} \\
\hline $40-59$ & 163 & $71 \%$ & 118 & $42 \%$ & \\
\hline $60+$ & 35 & $15 \%$ & 154 & $55 \%$ & \\
\hline \multicolumn{6}{|l|}{ Highest completed level of education ${ }^{a, b}$} \\
\hline Secondary school intermediate level or less & 60 & $26 \%$ & 135 & $48 \%$ & \multirow{3}{*}{$<0.001$} \\
\hline Medium vocational training (MBO), secondary school high level & 81 & $36 \%$ & 77 & $28 \%$ & \\
\hline Higher vocational training ( $\mathrm{HBO}) /$ university & 87 & $38 \%$ & 68 & $24 \%$ & \\
\hline
\end{tabular}

Table 1 continues on next page 


\begin{tabular}{|c|c|c|c|c|c|}
\hline \multicolumn{6}{|l|}{ Marital status $^{b}$} \\
\hline Married/living together & 180 & $79 \%$ & 202 & $72 \%$ & \multirow[t]{2}{*}{0.082} \\
\hline Divorced/partner deceased & 49 & $21 \%$ & 79 & $28 \%$ & \\
\hline \multicolumn{6}{|l|}{ Socio-economic status (SES) ${ }^{c}$} \\
\hline Low & 58 & $25 \%$ & 105 & $37 \%$ & \multirow{3}{*}{0.014} \\
\hline Medium & 93 & $41 \%$ & 99 & $35 \%$ & \\
\hline High & 77 & $34 \%$ & 78 & $28 \%$ & \\
\hline Comorbidities $^{b}$ & 61 & $27 \%$ & 102 & $36 \%$ & 0.020 \\
\hline \multicolumn{6}{|l|}{$B M I^{a, b, d}$} \\
\hline Healthy weight (BMI<25) & 153 & $67 \%$ & 123 & $44 \%$ & \multirow{3}{*}{$<0.001$} \\
\hline Overweight ( $25<=\mathrm{BMI}>30)$ & 59 & $26 \%$ & 100 & $36 \%$ & \\
\hline Obese (BMI>30) & 15 & $7 \%$ & 57 & $20 \%$ & \\
\hline Smoking ${ }^{\mathrm{b}}$ & 43 & $19 \%$ & 42 & $15 \%$ & 0.248 \\
\hline \multicolumn{6}{|c|}{ Tumour characteristics } \\
\hline \multicolumn{6}{|l|}{ Stage (clinical) $^{\mathrm{b}}$} \\
\hline 0 & 70 & $31 \%$ & 62 & $22 \%$ & \multirow{4}{*}{$<0.001$} \\
\hline I & 85 & $37 \%$ & 61 & $22 \%$ & \\
\hline II & 64 & $28 \%$ & 118 & $42 \%$ & \\
\hline III & 3 & $1 \%$ & 28 & $10 \%$ & \\
\hline \multicolumn{6}{|l|}{ Receptor status ${ }^{a}$} \\
\hline Triple negative & 12 & $5 \%$ & 21 & $7 \%$ & \multirow{5}{*}{0.347} \\
\hline Hormone-negative, Her2-positive & 10 & $4 \%$ & 20 & $7 \%$ & \\
\hline Hormone-positive, Her2-positive & 120 & $52 \%$ & 129 & $46 \%$ & \\
\hline Hormone-positive, Her2-negative & 84 & $37 \%$ & 104 & $37 \%$ & \\
\hline Unknown & 3 & $1 \%$ & 7 & $2 \%$ & \\
\hline \multicolumn{6}{|l|}{ Grade $^{b}$} \\
\hline Grade I & 34 & $15 \%$ & 32 & $11 \%$ & \multirow{3}{*}{0.012} \\
\hline Grade II & 98 & $43 \%$ & 129 & $46 \%$ & \\
\hline Grade III & 83 & $36 \%$ & 81 & $29 \%$ & \\
\hline \multicolumn{6}{|l|}{ Lymph node status } \\
\hline NO / unknown & 171 & $75 \%$ & 163 & $58 \%$ & \multirow[t]{2}{*}{$<0.001$} \\
\hline$>\mathrm{NO}$ & 58 & $25 \%$ & 118 & $42 \%$ & \\
\hline Multifocality & 78 & $34 \%$ & 63 & $22 \%$ & 0.010 \\
\hline \multicolumn{6}{|c|}{ Treatment characteristics } \\
\hline \multicolumn{6}{|l|}{ Mastectomy ${ }^{b}$} \\
\hline Bilateral therapeutic mastectomy & 14 & $6 \%$ & 14 & $5 \%$ & \multirow{3}{*}{$<0.001$} \\
\hline Therapeutic and contralateral prophylactic mastectomy & 41 & $18 \%$ & 11 & $4 \%$ & \\
\hline Unilateral therapeutic mastectomy & 174 & $76 \%$ & 256 & $91 \%$ & \\
\hline Radiotherapy & 39 & $17 \%$ & 95 & $34 \%$ & $<0.001$ \\
\hline Hormone therapy & 106 & $46 \%$ & 149 & $53 \%$ & 0.130 \\
\hline Chemotherapy & 106 & $46 \%$ & 143 & $51 \%$ & 0.301 \\
\hline Neoadjuvant chemotherapy & 26 & $11 \%$ & 47 & $17 \%$ & 0.085 \\
\hline
\end{tabular}

Table 1 continues on next page 


\begin{tabular}{|c|c|c|c|c|c|}
\hline & $\begin{array}{c}\text { IBR } \\
(n=229)\end{array}$ & $\%$ & $\begin{array}{c}\text { No IBR } \\
(n=281)\end{array}$ & $\%$ & p-value* \\
\hline \multicolumn{6}{|l|}{ Hospital characteristics } \\
\hline \multicolumn{6}{|l|}{ IBR hospital volume } \\
\hline Low & 22 & $10 \%$ & 51 & $18 \%$ & \multirow{3}{*}{0.001} \\
\hline Medium & 70 & $31 \%$ & 106 & $38 \%$ & \\
\hline High & 137 & $60 \%$ & 124 & $44 \%$ & \\
\hline \multicolumn{6}{|l|}{ Breast cancer surgery hospital volume ${ }^{f}$} \\
\hline Low & 74 & $32 \%$ & 114 & $40 \%$ & \multirow{3}{*}{0.125} \\
\hline Medium & 88 & $38 \%$ & 101 & $36 \%$ & \\
\hline High & 67 & $30 \%$ & 66 & $24 \%$ & \\
\hline \multicolumn{6}{|l|}{ Hospital type ${ }^{g}$} \\
\hline General hospital & 84 & $37 \%$ & 135 & $48 \%$ & \multirow{3}{*}{0.002} \\
\hline Top clinical hospital & 111 & $48 \%$ & 127 & $45 \%$ & \\
\hline Academic hospital (including breast cancer-specialized hospital) & 34 & $15 \%$ & 19 & $7 \%$ & \\
\hline
\end{tabular}

IBR: mastectomy with immediate breast reconstruction. No IBR: mastectomy without IBR. BMI: body mass index.

* Chi-square tested

a Totals do not match up due to missing values

${ }^{\mathrm{b}}$ self-reported

' Socio-economic status (SES) of the patients was based on four-digit postal code at time of surgery. SES-scores are provided by the Netherlands Institute for Social Research (Sociaal Cultureel Planbureau) and divided into three groups based on the delivered rank numbers: low (1st-3rd deciles), intermediate (4th-7th) and high (8th-10th) SES.

${ }^{d}$ Body Mass Index (BMI) based on body length and weight, according to WHO-definition.

e Percentage of annual IBR for mastectomy patients per hospital, categorised as low (0\% IBR), middle (1-15\%) or high volume (>15\%); respectively 12, 43, and 37 hospitals in the Netherlands had low, middle, and high volume IBR.

${ }^{\mathrm{f}}$ Number of surgical treated breast cancer patients per year (average over 2012-2014), categorised as low (<150), middle (150-249), and high (>250) volume.

${ }^{\mathrm{g}}$ Hospitals were categorised as either general, teaching, or academic hospitals (including breast cancer-specialized hospital).

\section{Shared decision-making for patients who had received IBR versus no IBR}

Patients with IBR compared to patients without IBR were more often preoperatively informed about the opportunity for IBR ( $99 \%$ vs $75 \%$; $p<0.001$ ), were just as often informed about DBR $(77 \%$ vs $73 \% ; p=0.534)$, and they were less often informed about the possibility of an external breast prosthesis to conceal the missing breast when dressed (64\% vs $81 \% ; p<0.001$ ).

Of all patients who had received preoperative information about IBR, $86 \%$ of the patients with IBR versus $68 \%$ of the patients without IBR had discussed the advantages and disadvantages of IBR with their physician $(p<0.001)$. Moreover, patients with IBR more often reported that the information about breast reconstruction had been comprehensible $(p<0.001)$ and they felt more often than patients without IBR they had had the opportunity to ask questions about breast reconstruction issues $(p<0.001)$. More patients with IBR $(91 \%)$ than patients without IBR $(67 \%)$ felt they had shared the decision-making with their physicians ( $<<0.001$; Table 2).

Most patients reported they had chosen their treatment based on their preferences (IBR: $53 \%$, without IBR 68\%) or their physician had recommended the received treatment (IBR: 41\%, without IBR: 10\%; Figure 1). 
For both patients with IBR and without IBR, $41 \%$ of patients who had been treated with radiotherapy had received a preoperative consultation with a radiation oncologist. Furthermore, for patients who were treated with radiotherapy and had received information about IBR, $63 \%$ of patients with IBR and $51 \%$ without IBR had been informed about the effects of radiotherapy on breast reconstruction ( $p=0.082$, Table 2$)$.

\section{Factors affecting receiving IBR}

The following variables were significantly related to having undergone IBR in univariate analyses and were therefore included in the multivariate analysis: informed about IBR, age, BMI $\geq 25$, highest completed education, two or more comorbidities, stage II or III tumour, multifocal tumour, bilateral mastectomy, neoadjuvant chemotherapy, radiotherapy, and IBR hospital volume.

In the multivariate logistic regression analyses, receiving IBR was significantly and positively affected by preoperatively being informed about IBR: these women had a 14 fold higher chance of receiving IBR (OR 13.87, $\mathrm{Cl}$ : $3.75-51.30$ ). Other significant factors were age over $60, \mathrm{BMI}$ over 25 , stage II and III, multifocality, bilateral mastectomy, and radiotherapy, and IBR hospital volume (Table 3).

Table 2: Patient-reported experience with information provision on treatment options, on pros and cons of treatment options, and shared decision-making about IBR

\begin{tabular}{|c|c|c|c|c|c|}
\hline & $\begin{array}{c}\text { IBR } \\
(n=229)\end{array}$ & $\%$ & $\begin{array}{l}\text { No IBR } \\
(n=281)\end{array}$ & $\%$ & $\mathrm{P}^{*}$ \\
\hline \multicolumn{6}{|c|}{ Information on available treatment options } \\
\hline \multicolumn{6}{|l|}{ Patient was preoperatively informed about possible treatment with: } \\
\hline IBR & 226 & $99 \%$ & 204 & $73 \%$ & $<0.001$ \\
\hline DBR & 176 & $77 \%$ & 204 & $73 \%$ & 0.534 \\
\hline External breast prosthesis & 147 & $64 \%$ & 228 & $81 \%$ & $<0.001$ \\
\hline \multicolumn{6}{|c|}{ Patient received information on pros and cons of reconstruction } \\
\hline \multirow{2}{*}{$\begin{array}{l}\text { Pros and cons of IBR were discussed between patient and } \\
\text { physician (if received information on IBR) }\end{array}$} & $n=226$ & & $n=204$ & & \\
\hline & 194 & $86 \%$ & 139 & $68 \%$ & $<0.001$ \\
\hline \multirow{2}{*}{$\begin{array}{l}\text { Patient regarded information about BR was comprehensible (if } \\
\text { information received about IBR) }\end{array}$} & $n=224$ & & $n=227$ & & \\
\hline & 224 & $100 \%$ & 191 & $85 \%$ & $<0.001$ \\
\hline \multirow{2}{*}{$\begin{array}{l}\text { Patient had opportunity to ask questions about BR } \\
\text { (if information received about IBR) }\end{array}$} & $n=226$ & & $n=204$ & & \\
\hline & 226 & $100 \%$ & 189 & $84 \%$ & $<0.001$ \\
\hline \multicolumn{6}{|c|}{ Discussing effects of radiotherapy on BR } \\
\hline \multirow{2}{*}{$\begin{array}{l}\text { Patient had a preoperative consultation with radiation oncologist } \\
\text { (if treated with radiotherapy) }\end{array}$} & $n=39$ & & $\mathrm{n}=95$ & & \\
\hline & 16 & $41 \%$ & 39 & $41 \%$ & 0.784 \\
\hline \multirow{2}{*}{$\begin{array}{l}\text { Patient received information on effect of radiotherapy on BR (if } \\
\text { information received about IBR and treated with radiotherapy) }\end{array}$} & $n=38$ & & $\mathrm{n}=74$ & & \\
\hline & 24 & $63 \%$ & 38 & $51 \%$ & 0.082 \\
\hline \multicolumn{6}{|c|}{ Experienced shared decision-making } \\
\hline Patient felt she could share the decision regarding $B R$ & 208 & $91 \%$ & 187 & $67 \%$ & $<0.001$ \\
\hline
\end{tabular}

IBR: mastectomy with immediate breast reconstruction. No IBR: mastectomy without IBR. BR: breast reconstructive surgery. DBR: delayed breast reconstruction.

*chi-square test 


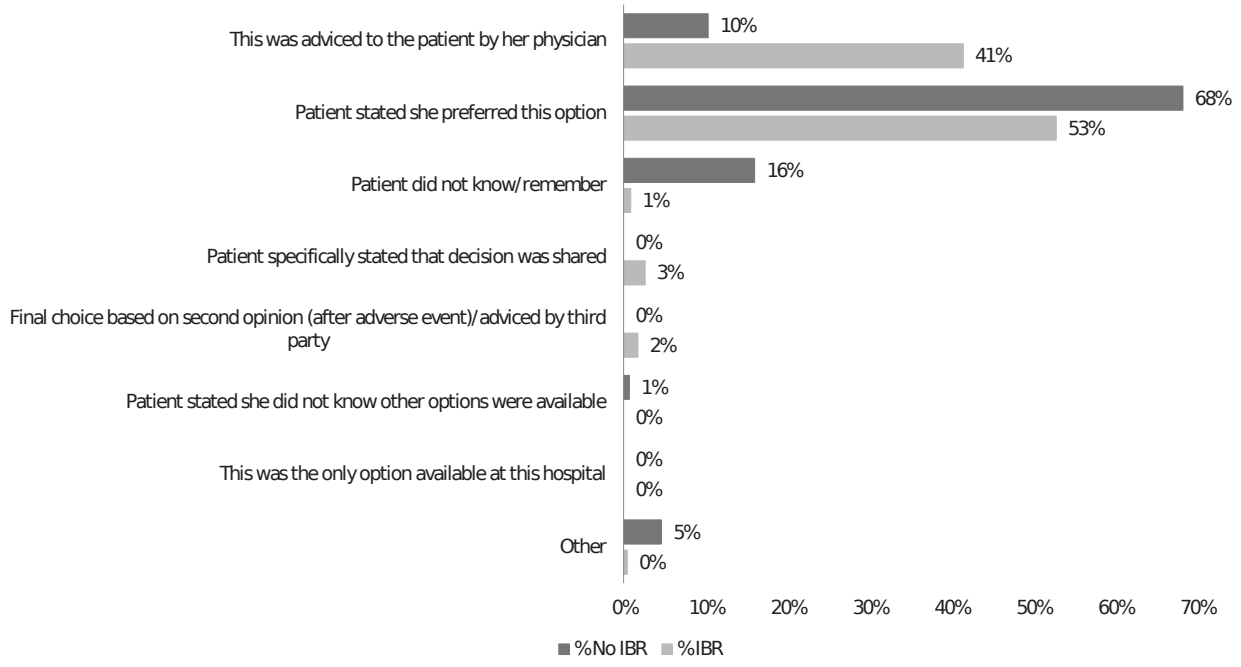

Figure 1: Patient-reported reasons for the choice for IBR, patients with IBR $(n=229)$ versus no IBR $(n=281)$. IBR: immediate breast reconstruction. No IBR: mastectomy without IBR

Table 3: Effect of information provision about IBR on receiving IBR, corrected for patient, tumour, and treatment characteristics

\begin{tabular}{|c|c|c|c|c|c|c|}
\hline & \multirow[t]{2}{*}{$\mathrm{n}$} & \multicolumn{2}{|c|}{ Univariable } & \multicolumn{2}{|c|}{ Multivariable } & \multirow[t]{2}{*}{$\mathrm{P}^{\mathrm{b}}$} \\
\hline & & OR & $95 \% \mathrm{Cl}$ & OR & $95 \% \mathrm{Cl}$ & \\
\hline \multicolumn{7}{|c|}{ Information about IBR } \\
\hline \multicolumn{7}{|c|}{ Patient received information about IBR } \\
\hline No/don't know & 90 & ref & & ref & & \\
\hline Yes & 444 & 28.43 & $8.84-91.51$ & 13.87 & $3.75-51.30$ & $<0.001$ \\
\hline \multicolumn{7}{|c|}{ Patient characteristics } \\
\hline \multicolumn{7}{|l|}{ Age $^{a}$} \\
\hline$<40$ & 44 & 2.49 & $1.14-5.43$ & 1.84 & $0.66-5.19$ & 0.246 \\
\hline $40-59$ & 295 & ref & & Ref & & \\
\hline $60+$ & 195 & 0.16 & $0.11-0.25$ & 0.21 & $0.12-0.36$ & $<0.001$ \\
\hline \multicolumn{7}{|l|}{ BMla } \\
\hline Healthy weight (BMI<25) & 286 & Ref & & Ref & & \\
\hline Overweight $(25<=\mathrm{BMI}>30)$ & 171 & 0.47 & $0.32-0.71$ & 0.59 & $0.35-1.01$ & 0.055 \\
\hline Obese (BMI>30) & 74 & 0.21 & $0.11-0.39$ & 0.22 & $0.10-0.48$ & $<0.001$ \\
\hline \multicolumn{7}{|l|}{ Comorbidities $^{\mathrm{a}}$} \\
\hline None & 351 & Ref & & Ref & & \\
\hline One & 139 & 0.67 & $0.44-1.01$ & 1.11 & $0.64-1.95$ & 0.708 \\
\hline Two or more & 33 & 0.42 & $0.19-0.94$ & 0.91 & $0.29-2.90$ & 0.879 \\
\hline
\end{tabular}

Table 3 continues on next page 


\begin{tabular}{|c|c|c|c|c|c|c|}
\hline \multicolumn{7}{|l|}{ Highest completed level of education ${ }^{a}$} \\
\hline $\begin{array}{l}\text { Secondary school intermediate level or } \\
\text { less }\end{array}$ & 203 & Ref & & Ref & & \\
\hline $\begin{array}{l}\text { Medium vocational training (MBO), } \\
\text { secondary school high level }\end{array}$ & 168 & 2.37 & $1.53-3.66$ & 1.54 & $0.86-2.78$ & 0.149 \\
\hline $\begin{array}{l}\text { Higher vocational training ( } \mathrm{HBO} \text { ) or } \\
\text { university }\end{array}$ & 160 & 2.88 & $1.85-4.47$ & 1.62 & $0.88-2.97$ & 0.123 \\
\hline \multicolumn{7}{|c|}{ Tumour characteristics } \\
\hline \multicolumn{7}{|l|}{ Stage (clinical) } \\
\hline Stage 0 (DCIS) & 135 & Ref & & Ref & & \\
\hline Stage I & 155 & 1.23 & $0.77-1.98$ & 1.17 & $0.64-2.14$ & 0.613 \\
\hline Stage II & 189 & 0.48 & $0.30-0.76$ & 0.39 & $0.20-0.75$ & 0.005 \\
\hline Stage III & 34 & 0.09 & $0.03-0.33$ & 0.08 & $0.01-0.41$ & 0.003 \\
\hline \multicolumn{7}{|l|}{ Multifocality } \\
\hline No & 386 & Ref & & Ref & & \\
\hline Yes & 148 & 1.79 & $1.21-2.64$ & 2.52 & $1.43-4.42$ & 0.001 \\
\hline \multicolumn{7}{|c|}{ Treatment characteristics } \\
\hline \multicolumn{7}{|l|}{ Mastectomy ${ }^{\mathrm{a}}$} \\
\hline Unilateral mastectomy & 448 & Ref & & Ref & & \\
\hline Bilateral mastectomy & 86 & 3.24 & $1.94-5.39$ & 2.22 & $1.08-4.55$ & 0.029 \\
\hline \multicolumn{7}{|l|}{ Axillary dissection } \\
\hline No & 128 & Ref & & - & - & - \\
\hline Yes & 406 & Omitted & Omitted & - & - & - \\
\hline \multicolumn{7}{|l|}{ Neoadjuvant chemotherapy } \\
\hline No & 460 & Ref & & Ref & & \\
\hline Yes & 74 & 0.63 & $0.37-1.07$ & 0.99 & $0.41-2.40$ & 0.994 \\
\hline \multicolumn{7}{|l|}{ Radiotherapy } \\
\hline No & 394 & Ref & & Ref & & \\
\hline Yes & 140 & 0.40 & $0.26-0.61$ & 0.52 & $0.28-0.98$ & 0.045 \\
\hline \multicolumn{7}{|c|}{ Hospital factors } \\
\hline \multicolumn{7}{|l|}{ IBR hospital volume ${ }^{\mathrm{C}}$} \\
\hline Low & 76 & 0.39 & $0.22-0.68$ & 0.43 & $0.20-0.94$ & 0.035 \\
\hline Middle & 186 & 0.60 & $0.41-0.88$ & 0.41 & $0.24-0.69$ & 0.001 \\
\hline High & 272 & Ref & & Ref & & \\
\hline
\end{tabular}

IBR: immediate breast reconstruction.

a Self-reported.

${ }^{\mathrm{b}}$ Chi-square tested.

c Hospital volume (\%IBR) was based on percentage of annual IBR for mastectomy patients, and categorised as low $(0 \%$ IBR), middle $(0-15 \%)$ or high volume $(>15 \%)$.

\section{Discussion}

The objectives of the current study were to gain insight into the underlying decision-making process of IBR and to determine the effect of preoperative information provision about IBR on receiving IBR. 
Based on more than five hundred completed questionnaires, we found that patients treated with IBR had been better informed about IBR, more often had weighed the advantages and disadvantages of IBR in discussion with their physician, and more often had experienced shared decision-making regarding IBR compared to patients without IBR. Furthermore, our multivariate logistic regression showed that being informed about IBR increased the probability for receiving IBR fourteen-fold. Because of our large sample, we were able to statistically control this relation for patient, tumour, treatment, and hospital factors.

Since we found that being informed about IBR had a large effect on receiving IBR, it may well be that the uninformed mastectomy patients would have opted for IBR if they had received information about IBR. A prospective study by Ananian et al. reported that patients who opted for breast reconstruction more frequently recognized the importance of discussing breast reconstruction with their surgeon, and women who had benefitted more frequently from discussions with their physician in general tended to prefer IBR over DBR [34]. Other factors that significantly reduced the chance of receiving IBR in the multivariate regression analysis, mainly were risk-factors for postoperative complications after IBR (age over 55, BMI over 30 , radiotherapy), as stated in the national guideline [17].

We found that patients without IBR were less often informed about IBR as a treatment option and its advantages and disadvantages than patients with IBR. However, it is the preoperative information discussed between patient and physician that is particularly considered carefully when making a choice $[34,35]$ and not so much the information the patient collects herself from other resources. Guidelines, including the Dutch guideline on breast reconstruction published in 2015 , already stress the importance of starting the discussion on different BR possibilities at the same time when mastectomy is offered to the patient by the surgeon $[17,36]$.

It has already been found that low satisfaction with preoperative information is associated with an increased likelihood of decisional regret [37]. However, in our questionnaire almost $70 \%$ of patients without IBR stated they preferred not to have IBR, and $10 \%$ indicated their physician advised this. After analysing the free text field in the survey of the latter patients, it became evident the advice to postpone breast reconstruction was based on severity of tumour characteristics or the increased risk of surgical complications. This suggests that mainly those patients with strong contra-indications for IBR were recommended mastectomy without IBR by their physician; therefore, we would be jumping to conclusions by merely stating that patients without IBR were not informed about IBR. Patients knowing about IBR as a treatment option, could have rejected this option immediately, therefore not receiving any further information. Contradictory to this, patients without IBR less often felt they shared the decision with their physician. Since patients without IBR in our study were older and lower educated, it could well be these patients did not want to share the decision, which was found in patients with these characteristics before [38, 39]. A previous study reported that surgeons tend to predict their patients' preferences fairly accurately [34]. For one fifth of the mastectomy patients in our study, the choice had not been based on their preferences or on a medical necessity; these patients possibly may have missed their chance of receiving IBR. As women vary in information seeking behaviour [40], tailoring 
information to individual patients [41] may be helpful here. Furthermore, we suggest that it should be documented in every patient's file whether and which breast reconstruction options were discussed; this is already recommended in the national guideline on breast reconstruction as well [17]. Often, this documentation is lacking [42], while it could help the physician in revealing unmet needs in patient information as well as education [42].

Harcourt et al. reported that only $15 \%$ of respondents searched for further information before making a decision, while $82 \%$ made 'instant' decisions (during the consultation where the reconstruction was first discussed) [35]. Opting for DBR creates a time span for patients in which they can explore their possibilities considering different breast reconstruction types and which provides professionals with the ability to assess whether patients are fully prepared for the outcomes [43]. Neo-adjuvant therapy creates a time span to surgery as well [43]. Based on our selection, we did not know on beforehand who had DBR or was considering this; it turned out 24 patients had had DBR. Thirty-eight respondents that had mastectomy without IBR in our study stated they were currently considering DBR; twenty-five of these patients $(70 \%)$ were informed about IBR before receiving mastectomy. Since time between diagnosis and completing the questionnaire was short for some patients, we presume that more mastectomy patients in our sample eventually will receive DBR.

We found that patients with IBR more often felt the decision-making had been shared between themselves and their physician compared to patients without IBR. Several studies have reported variation between actual and preferred involvement in decision-making for breast cancer treatment $[31,44,45]$. As described above, some mastectomy patients were denied IBR based on tumour or treatment characteristics. Therefore, they might feel they did not have a choice. However, involving patients in the decision-making process should always be promoted, because of its positive effects on quality of life [24] and patient satisfaction [29-31]. Although we did not measure SDM-preferences, we expect that a majority of patients would have wanted to participate in decision making [30,31]. Besides, Lee et al. reported that the majority of patients in their study felt involved in decision-making, while their knowledge on the procedure of IBR could be improved [46]; shared decision-making is therefore not the sole result of providing information.

Radiotherapy is an accepted reason to omit IBR [20,47], as it has been shown to increase the risk of implant loss, complications, poorer aesthetic results and less satisfied patients [48]. However, these increased complication and failure rates did not seem to apply for immediate autologous reconstructions, with comparable (partly unpublished) results for pre-reconstructive and postreconstructive exposure to radiation therapy $[49,50]$. Since implant-based reconstruction is the most applied form of breast reconstruction [48], which was applicable for our respondents as well, a majority of patients will face the trade-off between choosing IBR with a hazard of experiencing the negative effects of radiotherapy, or omitting IBR and therefore omitting its positive effects on quality of life and psychosocial functioning. Therefore, patients should be fully aware of these effects in order to make an informed decision. Interestingly, Flitcroft et al. reported that when patients (two-stage with tissue expander) were informed about potential negative aesthetic sideeffects of post-mastectomy radiotherapy on IBR, 63\% still opted for IBR [51]. In our study, 39\% of patients who had been informed about the effects of radiotherapy had undergone IBR. 


\section{Strengths and limitations}

With a response of over five hundred patients from a large geographically diverse and randomly selected nationwide sample, we believe that we accurately reflected reconstructive care for mastectomy patients in the Netherlands. This was confirmed by our analysis of the characteristics of respondents versus non-respondents. Furthermore, patient experiences and clinical data were combined, therefore creating a broad dataset for each patient.

However, also some limitations in the design of our study can be identified. Because patients had undergone mastectomy in 2013 or 2014, whereas our survey was conducted in 2015, potential bias lies in the patients' ability to properly recall the exact process of decision-making and information provision [52]. In addition, there may have been a reduction of inconsistencies between current beliefs and previous decisions ('cognitive dissonance reduction') [53]. Finally, inherent to an online survey, and despite the possibility to provide paper based questionnaires, respondents were younger than non-respondents, leading to a slightly lower response rate in the group without IBR. Since we used a non-validated questionnaire, not all invited patients responded, and respondents characteristics were not equal over both groups, which are limitations inherent to patient-reported data, interpretation of the results should be done with caution.

We recommend that every woman who faces mastectomy is informed about all relevant options for breast reconstruction [54]. She consequently also should receive this information, even if this means that she has to be referred to another hospital to undergo the type of IBR she desires. Only after knowing and understanding all options, a well-informed decision can be made by the patient. Ideally, all patients should be referred to a plastic surgeon for a completely balanced weighing of the decision whether or not to perform IBR, because another study recently conducted in the Netherlands revealed surgeons informed patients differently compared to plastic surgeons [50]. Physician education is important to accomplish continuity of care and proper referral. Furthermore, more implant IBR than autologous IBR can be performed within the same amount of time; thus, financial incentives made performing implant IBR more attractive for hospitals than autologous IBR [55], explaining the relative low number of respondents with autologous reconstruction. As IBR is covered by every patients' health insurance (except for the obligatory deductible excess of $€ 385$,- (2017))[56], this does not hinder access.

\section{Conclusions}

Patients who received IBR had been better informed about IBR as a treatment option, more often had discussed advantages and disadvantages of IBR, and felt significantly more involved in SDM than patients without IBR. After correction for patient, tumour, treatment, and hospital factors, being informed about IBR significantly increased the odds of receiving IBR fourteen-fold.

Our results highlight the importance of providing sufficient information on all relevant treatment options. 


\section{References}

1. Integraal Kankercentrum Nederland (IKNL). Cijfers over kanker: Incidentie Borst: Integraal Kankercentrum Nederland (IKNL); [selection: incidence, female, 2014, invasive and DCIS]. Available from: http://www. cijfersoverkanker.nl/selecties/incidentie_borst/img58b57441aa333.

2. Morrow M, Strom EA, Bassett LW, Dershaw DD, Fowble B, Giuliano A, et al. Standard for breast conservation therapy in the management of invasive breast carcinoma. CA Cancer J Clin. 2002;52:277-300.

3. Netherlands Comprehensive Cancer Organisation (IKNL). National guideline on Breast Cancer. Netherlands Comprehensive Cancer Organisation (IKNL),,; 2012.

4. Rowland JH, Desmond KA, Meyerowitz BE, Belin TR, Wyatt GE, PA G. Role of breast reconstructive surgery in physical and emotional outcomes among breast cancer survivors. J Natl Cancer Inst. 2000;92:1422-9.

5. Parker PA, Youssef A, Walker S, Basen-Engquist K, Cohen L, Gritz ER, et al. Short-term and long-term psychosocial adjustment and quality of life in women undergoing different surgical procedures for breast cancer. Ann Surg Oncol. 2007;14:3078-89.

6. Cordeiro PG. Breast Reconstruction after Surgery for Breast Cancer. New England Journal of Medicine. 2008(359):1590-601.

7. Elder EE, Brandberg Y, Björklund T, Rylander R, Lagergren J, Jurell G, et al. Quality of life and patient satisfaction in breast cancer patients after immediate breast reconstruction: a prospective study. Breast (Edinburgh, Scotland). 2005;14(3):201-8.

8. Howard MA, Polo K, Pusic AL, Cordeiro PG, Hidalgo DA, Mehrara B, et al. Breast cancer local recurrence after mastectomy and TRAM flap reconstruction: incidence and treatment options. Plast Reconstr Surg. 2006;117(5):1381-6.

9. Robb GL. Reconstructive Surgery. In: Hunt KK, Robb GL, Strom EA, Ueno NT, editors. Breast Cancer. New York: Springer; 2001. p. 223-53.

10. Zhang $\mathrm{P}$, Li CZ, Wu CT, Jiao GM, Yan F, Zhu HC, et al. Comparison of immediate breast reconstruction after mastectomy and mastectomy alone for breast cancer: A meta-analysis. European journal of surgical oncology : the journal of the European Society of Surgical Oncology and the British Association of Surgical Oncology. 2017;43(2):285 - 93.

11. Yang X, Zhu C, Gu Y. The Prognosis of Breast Cancer Patients after Mastectomy and Immediate Breast Reconstruction: A Meta-Analysis. PLOS ONE. 2015;10(5).

12. Murphy RX Jr, Wahhab S, Rovito PF, Harper G, Kimmel SR, Kleinman LC, et al. Impact of immediate reconstruction on the local recurrence of breast cancer after mastectomy. Ann Plast Surg. 2003;50(4):333-8.

13. van Bommel ACM, Mureau MAM, Schreuder K, van Dalen T, Vrancken Peeters MTFD, Schrieks M, et al. Large variation between hospitals in immediate breast reconstruction rates after mastectomy for breast cancer in the Netherlands. Journal of Plastic, Reconstructive \& Aesthetic Surgery. 2017;70(2):215-21.

14. Jeevan R, Mennie JC, Mohanna PN, O’Donoghue JM, Rainsbury RM, DA. C. National trends and regional variation in immediate breast reconstruction rates. British Journal of Surgery. 2016;103:1147-56.

15. Dutch Institute for Clinical Auditing (DICA). Nabon Breast Cancer Audit - NBCA: Dutch Institute for Clinical Auditing (DICA),,; [Available from: https://www.dica.nl/nbca/home.

16. Schreuder K, Van Bommel ACM, De Ligt KM, Maduro JH, Vrancken Peeters MTFD, Mureau MAM, et al. Hospital organizational factors affect the use of immediate breast reconstruction after mastectomy for breast cancer in the Netherlands. Breast (Edinburgh, Scotland). 2017;34:96-102.

17. Mureau MAM, Nederlandse Vereniging voor Plastische Chirurgie (NVPC). Dutch Breast Reconstruction Guideline. Journal of Plastic Reconstructive and Aesthetic Surgery 2018:290-304.

18. Légaré F, Witteman HO. Shared Decision Making: Examining Key Elements And Barriers To Adoption Into Routine Clinical Practice. Health Affairs. 2013;32(2):276-84.

19. Cohen WA, Ballard TNS, Hamill JB, Kim HM, Chen XBS, Klassen A, et al. Understanding and Optimizing the Patient Experience in Breast Reconstruction. Annals of Plastic Surgery. 2016;77(2):237-41.

20. Nelson JA, Fischer JP, Radecki MA, Pasick C, McGrath J, Serletti JM, et al. Delayed autologous breast reconstruction: factors which influence patient decision making. J Plast Reconstr Aesthet Surg. 2013;66(11):1513-20. 
21. Mátrai Z, Kenessey I, Sávolt A, Ujhelyi M, Bartal A, Kásler M. Evaluation of patient knowledge, desire, and psychosocial background regarding postmastectomy breast reconstruction in Hungary: a questionnaire study of 500 cases. Med Sci Monit. 2014;12(20):2633-42.

22. Shekhawat L, Busheri L, Dixit S, Patel C, Dhar U, Koppiker C. Patient-Reported Outcomes Following Breast Reconstruction Surgery and Therapeutic Mammoplasty: Prospective Evaluation 1 Year Post-Surgery with BREAST-Q Questionnaire. Indian J Surg Oncol. 2015;6(4):356-62.

23. Kim MK, Kim T, Moon HG, Jin US, Kim K, Kim J, et al. Effect of cosmetic outcome on quality of life after breast cancer surgery. European journal of surgical oncology : the journal of the European Society of Surgical Oncology and the British Association of Surgical Oncology. 2014;41(3):426-32.

24. Hack TF, Degner LF, Watson P, L. S. Do patients benefit from participating in medical decision making? Longitudinal follow-up of women with breast cancer. Psychooncology. 2006;15(1):9-19.

25. Nissen MJ, Swenson KK, Kind EA. Quality of Life After Postmastectomy Breast Reconstruction. Oncology Nursing Forum. 2002;29(3).

26. Al-Ghazal SK, Fallowfield L, RW. B. Comparison of psychological aspects and patient satisfaction following breast conserving surgery, simple mastectomy and breast reconstruction. European journal of cancer (Oxford, England : 1990). 2000;36(15):1938-43.

27. Wilkins EG, Cederna PS, Lowery JC, Davis JA, Kim HM, Roth RS, et al. Prospective analysis of psychosocial outcomes in breast reconstruction: one-year postoperative results from the Michigan Breast Reconstruction Outcome Study. Plast Reconstr Surg. 2000;106(5):1014-25.

28. Al-Ghazal SK, Sully L, Fallowfield L, Blamey RW. The psychological impact of immediate rather than delayed breast reconstruction. European journal of surgical oncology : the journal of the European Society of Surgical Oncology and the British Association of Surgical Oncology. 2000;26(1):17-9.

29. Mandelblatt JS, Edge SB, Meropol NJ, Senie R, Tsangaris T, Grey L, et al. Predictors of Long-Term Outcomes in Older Breast Cancer Survivors: Perceptions Versus Patterns of Care. J Clin Oncol. 2003;21(5):855-63.

30. Brown R, Butow P, Wilson-Genderson M, Bernhard J, Ribi K, I. J. Meeting the Decision-Making Preferences of Patients With Breast Cancer in Oncology Consultations: Impact on Decision-Related Outcomes. J Clin Oncol. 2012;30(8).

31. Gattellari M, Butow PN, Tattersall MH. Shared decisions in cancer care. Soc Sci Med. 2001;52(12):1865-78.

32. van de Poll-Franse LV, Horevoorts N, Eenbergen M, Denollet J, Roukema JA, Aaronson NK, et al. The Patient Reported Outcomes Following Initial treatment and Long term Evaluation of Survivorship registry: Scope, rationale and design of an infrastructure for the study of physical and psychosocial outcomes in cancer survivorship cohorts. European journal of cancer (Oxford, England : 1990). 2011;47(14):2188-94.

33. StataCorp. Stata Statistical Software: Release 14. In: College Station TSL, , editor. 2015.

34. Ananian $P$, Houvenaeghel $G$, Protière $C$, Rouanet $P$, Arnaud S, Moatti JP, et al. Determinants of Patients' Choice of Reconstruction with Mastectomy for Primary Breast Cancer. Ann Surg Oncol. 2004;11(8):762-71.

35. Harcourt D, Rumsey N. Mastectomy patients' decision-making for or against immediate breast reconstruction. Psychooncology. 2004;13:106-15.

36. Zhong T, Spithoff K, Kellett S, Boyd K, Brackstone M, Hanrahan R, et al. Breast cancer reconstruction surgery (immediate and delayed) across Ontario: Patient indications and appropriate surgical options. Section 1: Recommendations. Ontario: Ontario Cancer Care: Action Cancer Ontario; 2016.

37. Sheehan J, Sherman KA, Lam T, J. B. Association of information satisfaction, psychological distress and monitoring coping style with post-decision regret following breast reconstruction. Psychooncology. 2007;16(4):342-51.

38. Janz NK, Wren PA, Copeland LA, Lowery JC, Goldfarb SL, Wilkins EG. Patient-Physician Concordance: Preferences, Perceptions, and Factors Influencing the Breast Cancer Surgical Decision. J Clin Oncol 2004;22(15):3091-8.

39. Wallberg B, Michelson H, Nystedt M, Bolund C, Degner LF, Wilking N. Information Needs and Preferences for Participation in Treatment Decisions Among Swedish Breast Cancer Patients. Acta Oncol. 2000;39(4):467-76.

40. Begum S, Grunfeld EA, Ho-Asjoe M, Farhadi J. An exploration of patient decision-making for autologous breast reconstructive surgery following a mastectomy. Patient Educ Couns. 2011;84(1):105-10. 
41. Temple-Oberle C, Ayeni O, Webb C, Bettger-Hahn M, Ayeni O, Mychailsychyn D. Shared Decision-Making: Applying a Person-Centered Approach to Tailored Breast Reconstruction Information Provides High Satisfaction Across a Variety of Breast Reconstruction Options. Journal of surgical oncology. 2014;110:796800.

42. Ogrodnik A, MacLennan S, Weaver D, James T. Barriers to completing delayed breast reconstruction following mastectomy: a critical need for patient and clinician education. J Canc Educ. 2016;32(4):700-6.

43. Greenall MJ. The timing of breast reconstruction. Ann R Coll Surg Engl. 2006;89:754-9.

44. Hawley ST, Lantz PM, Janz NK, Salem B, Morrow M, Schwartz K, et al. Factors associated with patient involvement in surgical treatment decision making for breast cancer. Patient Educ Couns 2007;65:387-95.

45. Vogel BA, Helmes AW, Hasenburg A. Concordance between patients' desired and actual decision-making roles in breast cancer care. psychooncology. 2008;17:182-289.

46. Lee CN, Ubel PA, Deal AM, Burdick Blizard L, Sepucha KR, Ollila DW, et al. How Informed is the Decision about Breast Reconstruction after Mastectomy? A Prospective, Cross-sectional Study. Ann Surg. 2016;264(6):11039.

47. Musgrave KJ, Bochner M, Kollias J. Surgical Decision-making in Immediate Breast Reconstruction. World J Surg. 2010;34:3029 - 35.

48. Magill LJ, Robertson FP, Jell G, Mosahebi A, Keshtgar M. Determining the outcomes of post-mastectomy radiation therapy delivered to the definitive implant in patients undergoing one- and two-stage implantbased breast reconstruction: A systematic review and meta-analysis. JPRAS. 2017;70:1329-35.

49. Jagsi R, Li Y, Morrow M, Janz N, Alderman A, Graff J, et al. Patient-Reported Quality of Life and Satisfaction with Cosmetic Outcomes After Breast Conservation and Mastectomy with and without Reconstruction: Results of a Survey of Breast Cancer Survivors. Ann Surg. 2015;261(6):1198-206.

50. Van Bommel ACM, Schreuder K, Veenstra RK, De Ligt KM, Vrancken Peeters MTFD, Maduro JH, et al. Discrepancies between surgical oncologists and plastic surgeons in patient information provision and personal opinions towards immediate breast reconstruction. Unpublished results.

51. Flitcroft K, Brennan M, Costa D, Wong A, Snook K, Spillane A. An evaluation of factors affecting preference for immediate, delayed or no breast reconstruction in women with high-risk breast cancer. Psycho-Oncology. 2016;25:463-9.

52. Wolf $L$. The information needs of women who have undergone breast reconstruction. Part II: Information giving and content of information. Eur J Oncol Nurs 2004;8:315-24.

53. Festinger L. Conflict, Decision, and Dissonance. Stanford, CA: Stanford University Press; 1964.

54. Wolf $L$. The information needs of women who have undergone breast reconstruction. Part I: decision-making and sources of information. European Journal of Oncology Nursing. 2004;8:211-23.

55. M E. Borstreconstructie steeds vaker met eigen weefsel. Volkskrant. 201318 September 2013.

56. ZorgverzekeringWijzer.nl. Vergoeding plaatsen van borst(mamma)prothesen 20182017 [Available from: https://www.zorgverzekeringwijzer.nl/zorgvergoedingen/plaatsen-van-borstmammaprothesen. 


\section{Appendix 1: Power calculation}

The power calculation for this questionnaire was based on the Breast- $Q$ modules that were included in our questionnaire, since the questions for these modules have been validated. The analysis for these questions however will be conducted in a separate manuscript.

Based on Zhong et al (2011) ${ }^{1}$, an SD of about 20 was found for separate Breast-Q subscales. A difference of 10 points on each scale (0-100) was considered clinically relevant. With an alpha of 0.05 (double sided) and a power of 0.85 , we needed 32 respondents in each group. Since we estimated we could invite a lot more patients, we chose a lower difference in points:

$h=0,50$ à $n=32$ (difference of 10 points)
$h=0,40$ à $n=49$ (difference of 6 points)
$h=0,25$ à $n=126$ (difference of 5 points)
$h=0,20$ à $n=197$ (difference of 4 points)

Furthermore, we expected a response of about $25 \%$. Therefore, we decided to invited 500 patients per group $(500 * 0,25=125)$. Since we wanted a sample of patients that were treated in all types of hospitals and all regions in the Netherlands, we decided to include at least 20 different hospitals. This meant that 50 patients had to be selected per participating hospital.

We hypothesize that selecting 50 patients per hospital leaves the required workload per hospital acceptable, since all patients are selected in deliberation with the physician and each hospital has to prepare letters for all selected patients, without receiving a financial incentive.

Since more than 20 hospitals committed to our study, we decided to include these hospitals as well; therefore, we were able to indicate differences of 4 points on the Breast- $Q$ subscales.

1. Zhong T, McCarthy C, Min S, Zhang J, Beber B, Pusic AL, Hofer SOP. Patient Satisfaction and Health-Related Quality of Life After Autologous Tissue Breast Reconstruction. Cancer, 2011. 118(6). P: 1701-1709. DOI: 10.1002/cncr.26417. 


\section{Appendix 2}

The following questions are covering understated subjects:

1 Questions on health and treatment:

General health: questions 1, 2.

Breast cancer treatment: questions 3-5.

Breast reconstruction: questions 17-21.

\section{Shared decision-making aspects:}

Both patient and physician acknowledge a decision is required (by knowing that Immediate Breast Reconstruction (IBR) is an option): questions 7-9, 16.

Understanding and weighing all available information about the treatment options: questions $10,12,13 ; 6,15$.

Incorporating the patients preferences in the final decision: questions 11, 22-24.

\section{Questions on patients background:}

Date of birth, nationality, educational level, working status, relationship status, breast size, body length and weight, and menopausal status: questions 46-57.

1. How was, in your own perception, your physical health over the past three months?

Excellent - very well - well - moderate - bad

2. How was, in your own perception, your mental health over the past three months?

Excellent - very well - well - moderate - bad

3. What kind of surgery did you receive?

Amputation - Amputation including axillary lymph node dissection - Other

4. When did you receive this surgical procedure?

[fill in date DD/MM/YYYY]

5. Did you receive surgery on one or both breast?

Both breast (double-sided) because of cancer in both breast - both breast (double-sided) because of cancer in a single breast while other side preventive - single breast (single-sided)

6. Did you have a consultation with a radiotherapist prior to your surgery?

Yes - no - can't remember - not applicable since I did not receive radiotherapy

7. Were you informed about possible treatment with an immediate breast reconstruction prior to receiving your surgical treatment?

Yes - no - can't remember

8. Were you informed about possible treatment with a delayed breast reconstruction prior to receiving your surgical treatment?

Yes - no - can't remember

9. Were you informed about the possibility of breast prosthesis prior to receiving your surgical treatment (when not opting for breast reconstruction)?

Yes - no - can't remember 
10. Did you and your physician discuss the pros and cons of an immediate breast reconstruction? Yes - no - not applicable: did not receive any information on IBR - can't remember

11. Do you feel you had a choice in choosing for either an immediate breast reconstruction, a delayed breast reconstruction, or no breast reconstruction at all?

Yes - no - not applicable: did not receive any information on IBR - can't remember

12. Do you feel the information you received about breast reconstruction was comprehensible? Yes - no - not applicable: did not receive any information on BR - can't remember

13. Do you feel you got the opportunity to ask your physician questions about breast reconstruction? Yes - no - not applicable: did not receive any information on BR - can't remember

14. Are you familiar with the 'B-bewust checklist', developed by the Dutch Breast Cancer Patient Association, and did you use this checklist when informing yourself about breast reconstructive procedures?

Yes, and applied this as well - yes, but did not apply this checklist - no - can't remember

15. Were you informed about the possible effects of irradiation on the possibility of receiving (any kind of) breast reconstructive procedure?

Yes - no - not applicable: did not receive any information on BR - can't remember

16. Were you redirected to another hospital in order to receive breast reconstructive surgery?

Not applicable, breast reconstruction was offered in my treating hospital - no, I was not redirected and did not receive a breast reconstructive surgery - no, I was not redirected but informed myself and eventually rejected breast reconstruction - no, I was not redirected but informed myself and eventually opted for breast reconstruction - yes, I was redirected and received breast reconstructive surgery - yes, I was redirected but rejected breast reconstructive surgery - I can't remember - other

17. Did you receive a breast reconstructive surgery, and if so, when did you receive this procedure?

I did not receive breast reconstructive surgery - I did not receive breast reconstructive surgery, but consider having this procedure in de (nearby) future - I did not receive breast reconstructive surgery, but a delayed breast reconstruction is currently planned on [date DD/MM/YYYY] - Yes, I received a delayed reconstruction on [date $\mathrm{DD} / \mathrm{MM} / \mathrm{YYYY}$ ] - Yes, I received a direct reconstruction

18. Which type of immediate breast reconstruction did you receive?

Tissue expander, followed by final prosthesis - Immediate final prosthesis without use of a tissue expander - Tissue from the back (latissimus dorsi) combined with final prosthesis, whether or not with use of tissue expander - tissue from the abdomen (TRAM, DIEP, SIEA) - tissue from thigh (TMG, SGAP, IGAP of PAP) - I don't know - Other

19. Which type of delayed breast reconstruction did you receive?

Tissue expander, followed by final prosthesis - Immediate final prosthesis without use of a tissue expander - Tissue from the back (latissimus dorsi) combined with final prosthesis, whether or not with use of tissue expander - tissue from the abdomen (TRAM, DIEP, SIEA) - tissue from thigh (TMG, SGAP, IGAP of PAP) - I don't know - Other

20. Did you receive a nipple reconstruction? 
Yes, a nipple reconstruction including medical tattooing of the areola - Yes, solely a nipple reconstruction - yes, solely medical tattooing of the areola - No, neither a nipple reconstruction nor medical tattooing of the areola - no, because my nipple was spared during amputation (autologous use for reconstructed breast)

21. Did you receive additional surgical procedures in order to accomplish an optimal result of your breast reconstruction?

No, I did not receive additional surgical procedures - Yes, I received additional surgical procedures for my reconstructed breast - Yes, I received additional surgical procedures for my preserved breast - Yes, I received additional surgical procedures for both breast - other

22. Why did you opt for an immediate breast reconstructive surgery?

My physician recommended this, without specifically stating why - My physician recommended this, because [open text field] - by own choice, because [open text field] - I did not know there were other options than an immediate breast reconstructive surgery - I don't know - other

23. Why did you opt for a delayed breast reconstructive surgery?

My physician recommended this, because an immediate breast reconstruction was to risky - My physician recommended this, because an immediate breast reconstruction was not a possibility since [ open text field] - My physician recommended this, without specifically stating why - My physician recommended this, because [open text field] - I did not know there were other options than an delayed breast reconstructive surgery - my treating hospital did only offer delayed breast reconstructive surgery - by own choice, because immediate reconstruction gave a high risk on complications - by own choice, since there was a waitlist to receive an immediate reconstructive surgery - by own choice, since I wanted to focus on getting better first - by own choice, since [ open text field] - I don't know - other

24. Why did you reject breast reconstructive surgery?

My physician recommended this, because a breast reconstruction gave too high risk on complications - My physician recommended this, without specifically stating why - My physician recommended this, because [open text field] - by own choice, because a reconstruction gave a high risk on complications by own choice, because I think a breast reconstructive surgery is unnecessary - by own choice, because I think receiving breast reconstructive surgery is a too lengthy procedure - by own choice, because I think a breast reconstructive surgery is too invasive - by own choice, because I think a breast reconstructive surgery leads to a lengthy and intensive recovery - by own choice, since [ open text field] - I did not know there were options for breast reconstructive surgery - I don't know - other

\section{BREAST-Q MODULES}

25 to 39: BREAST-Q Postoperative reconstruction module

40 to 45: BREAST-Q Postoperative mastectomy module

46. What is your date of birth?

..-.-19..

47. At time of your breast cancer treatment, what were the four digits of your postal code?

48. What is your highest completed education? (completed with diploma or certificate) No education - lower education - middle education - higher education - other

49. What is currently your marital status?

Married/relationship - divorced/separated - widow/widower/partner diseased - single 
50. Which description is most applicable to you at this moment?

Attending school/education - paid employment - unemployed/seeking work - incapacitated housewife - retirement

51. What is your nationality?

Dutch - Moroccan - Surinamese - Turkish - German - Belgian - Other

52. At time of your breast cancer surgery, how tall were you and how much did you weigh?

[ height in $\mathrm{cm}$ ]

[ weight in $\mathrm{kg}$ ]

53. At time of your breast cancer diagnosis, did you smoke?

Yes - no

54. At time of your treatment for breast cancer, did you suffer from one or more of undermentioned diseases?

Any other type of cancer - lung disease - cardiovascular disease - gastro-intestinal disease - illness of urinary or reproductive system - musculoskeletal disease - central nerve system - illness of metabolism or coagulopathy - infectious disease - none - other

55. What was your breast size at the time of your breast cancer diagnosis?

$A A-A-B-C-D-E-F-G-$ Other

56. Were you menopausal at time of your treatment with chemotherapy?

Pre-menopausal - peri-menopausal - post-menopausal - I don't know - not applicable

57. Are you an active member of an patient association for breast cancer or breast disease?

No - Yes, but not an active member - Yes, active member

Do you have any questions/remarks? 



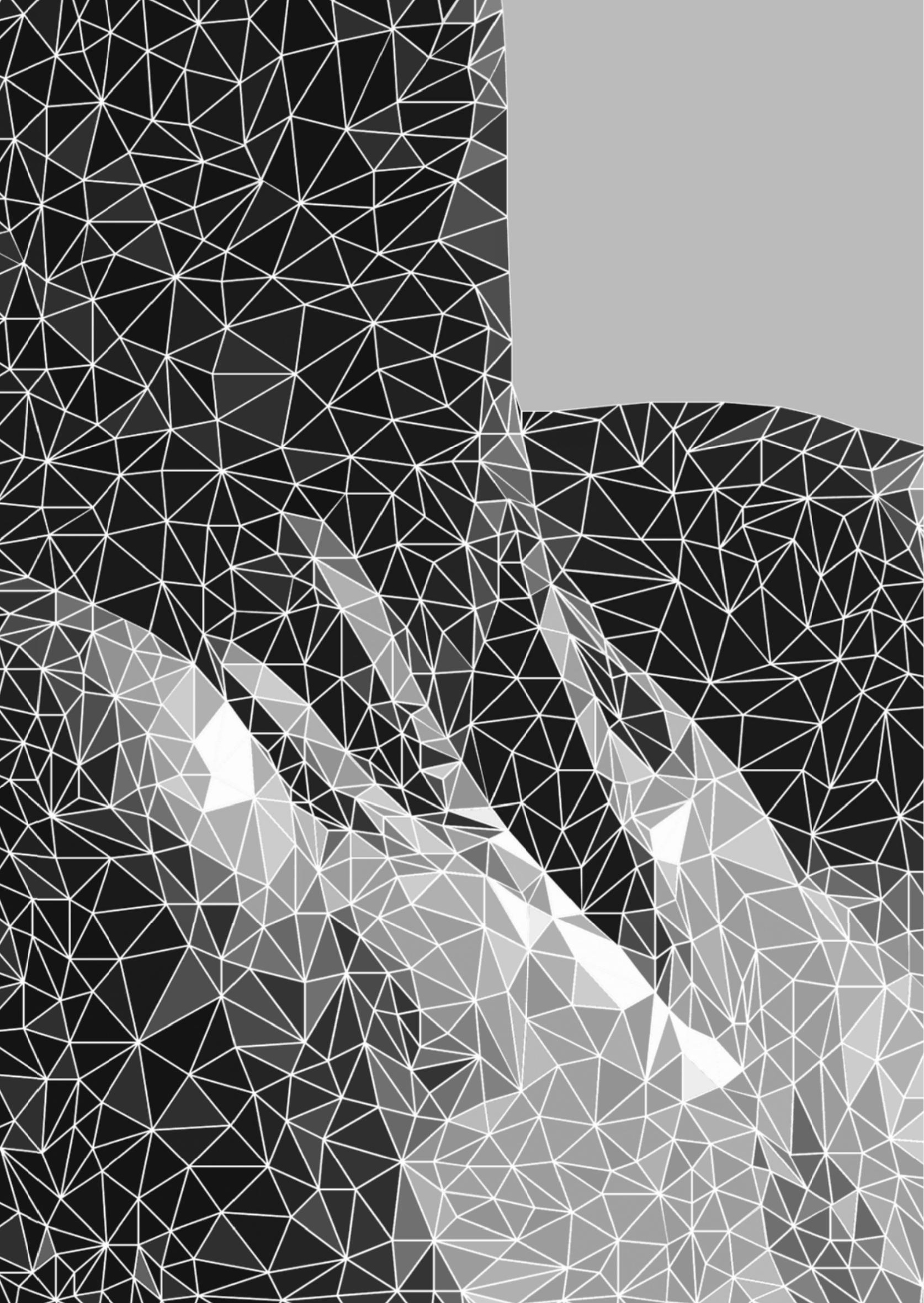


THIRD PART

POTENTIAL FOR PERSONALISATION OF FOLLOW-UP 


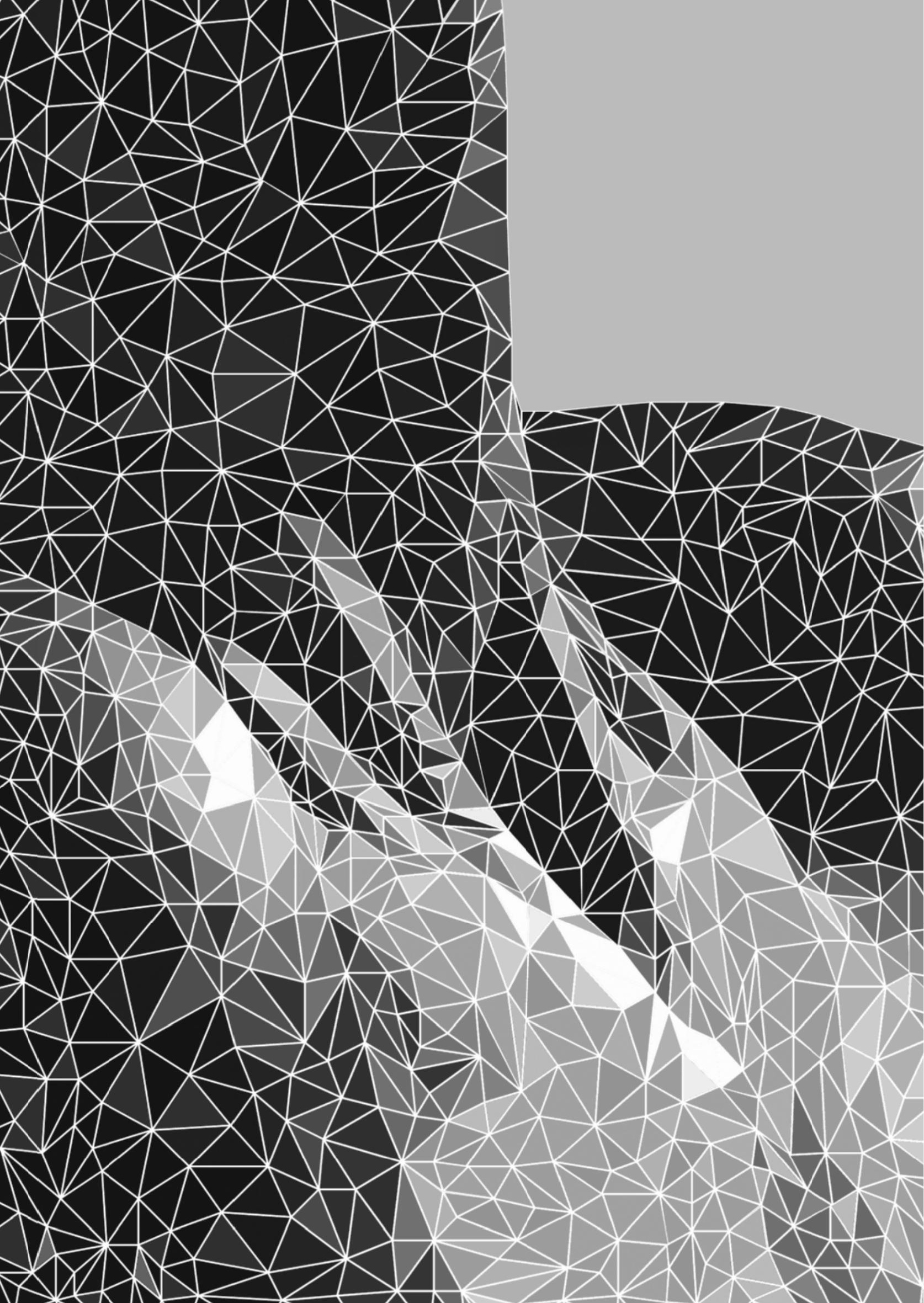




\section{CHAPTER 5}

PATIENT-REPORTED HEALTH PROBLEMS AND HEALTHCARE USE AFTER TREATMENT FOR EARLY-STAGE BREAST CANCER

K.M. de Ligt M. Heins

J. Verloop

C.H. Smorenburg J.C. Korevaar

S. Siesling

Breast. 2019 Apr 4;46:4-11. 



\section{Abstract}

\section{Background:}

A clear picture of treatment-related health problems following breast cancer treatment is useful in anticipating the informational and other needs of patients during follow-up. This study aimed to identify treatment-related health problems in breast cancer patients up to five years after diagnosis. Secondly, the use of care associated with these health problems was identified.

\section{Methods:}

876 surgically-treated female patients diagnosed between 2012-2016 with early-stage breast cancer were asked to complete an online survey about their current health problems and use of care. Multivariate logistic regression analyses were applied to determine the effect of patient and treatment characteristics on health problems.

\section{Results:}

404 patients responded (46\%). The median age was 62.0 years (SD:10.9). Apart from breast surgery, patients had been treated with radiotherapy (72\%), chemotherapy (49\%), anti-hormonal therapy (57\%), and axillary dissection (21\%). Ninety-three percent experienced one or more health problems. Over $50 \%$ of respondents experienced fatigue, psychological problems, and health problems regarding the breast, and/or musculoskeletal, central nervous, and reproductive system. Treatment with chemotherapy was significantly associated $(p<0.05)$ with an increased risk of health problems, respectively fatigue (OR:2.00), respiratory (OR:1.81), gastrointestinal (OR:1.87), central nervous (OR:3.40), and skin problems (OR:2.62). Use of healthcare for one or more health problems was reported by $64 \%$ of respondents.

\section{Discussion:}

Almost all patients experienced health problems up to five years after breast cancer diagnosis, with a range of complaints that were consistently present over time. Factors associated with the development of health problems are useful for better informing patients beforehand and targeting follow-up care. 


\section{Introduction}

Breast cancer and its treatment have a major impact on psychosocial, emotional, cognitive, and physical well-being [1-3], partly due to treatment-induced effects later. Surgery (including axillary resection) is associated with lymphoedema, pain in the arm and shoulder, and movement restrictions of the arm and shoulder [4]. Radiotherapy can lead to pneumonitis, skin changes, and pain and lymph oedema in the arm following nodal irradiation [4, 5]. Chemotherapy can lead to premature menopause, neuropathy [4] and bone loss [5]. Both chemotherapy and anti-hormonal therapy were also negatively associated with cognitive decline, although the evidence is not conclusive [4, 6]. Cardiotoxic effects can follow radiotherapy, chemotherapy (anthracyclines) and targeted therapies (anti-HER2 treatment and trastuzumab) [4, 5, 7, 8]. Other common effects of breast cancer diagnosis and treatment were fatigue, insomnia, depression and cognitive dysfunction $[1,4,6]$, and one third of patients experienced sexual problems, which were more pronounced in patients who were treated with chemotherapy [4].

All in all, the transition from treatment to 'life after cancer' is a difficult one, and can result in unmet needs [9]. Patients receive follow-up care to inform and counsel them about these potential physical and psychosocial late effects of the treatment and to detect these effects at an early stage [10-12]. International guidelines prescribe that follow-up visits should take place several times per year up to five years after completing treatment [10-12]. These visits are made in a hospital care setting, are often related to monitoring for recurrences of breast cancer and are done by a physician or nurse specialising in breast cancer care [10].

Although knowledge about (late) treatment-related effects is available for each treatment modality, several studies concluded that providing information to patients during follow-up could be improved: patients were often unaware of what effects to expect later and the duration of these effects, and thus felt unprepared for the post-treatment period [3, 13-15]. Patients also have expressed strong unmet needs for education, information, or support related to physical impairments or activity limitations [3]. A complete overview of risks of developing health problems after each of the breast cancer treatment modalities is useful in anticipating the informational and other needs of patients during the follow-up period. The primary goal of this study was to identify treatment-related health problems in breast cancer patients up to five years after completing curative treatment. A secondary aim was to identify use of care associated with these health problems.

\section{Materials and methods}

\section{Study population}

Twenty hospitals in the Netherlands ( 8 general hospitals, 10 teaching hospitals, and 2 academic hospitals) were willing to take part by inviting patients to fill in an online survey. In each participating hospital, fifty patients were selected from the Netherlands Cancer Registry (NCR). The NCR had documented data about incidence, diagnosis, and treatment since 1989 for all cancer 
patients treated in Dutch hospitals [16]. Those selected for inclusion were surgically-treated female breast cancer patients, diagnosed with early stage disease (stages I to III), aged 18 and older and diagnosed between 2012 and 2016. Patients were excluded if they were undergoing treatment for recurrent disease, could not read or write Dutch, were not receiving active follow-up anymore, or if their contact information was not up to date $(n=124)$. Paper questionnaires were provided on request for patients without computer access.

From September 2017 to March 2018, 876 patients were invited by letter through the patient administration systems of each participating hospital; responses were collected until May 2018. Each letter contained individual log in credentials for the PROFILES Registry ('Patient Reported Outcomes Following Initial treatment and Long-term Evaluation of Survivorship'), an online survey application developed to study the physical and psychosocial impact of cancer and its treatment from a cohort of cancer survivors [17]. On first using this application, patients set up their own password and give consent for their responses to be processed and merged with their clinical data in the NCR. The Dutch Medical Research (Human Subjects) Act does not apply for this type of study and formal approval from an ethics committee was not required. The use of NCR data in this study was approved by the NCR Privacy Review Board.

\section{Questionnaire}

The self-administered survey (Appendix 1) consisted of three topics: 1) a predefined list of health problems experienced during the twelve months preceding the questionnaire, retrieved from Yzermans et al. [18]; 2) associated use of health care (visiting a physician or general practitioner) over the last year; 3 ) personal information including highest completed level of education, current treatment status, and comorbidities. Comorbidity was defined as chronic health problems that patients experienced at the time of completing the survey, including other types of cancer, pulmonary, cardiovascular, gastrointestinal, urogenital, musculoskeletal, neurological, metabolic/ coagulation, or infectious diseases. The questionnaire was tested by a panel of current and former breast cancer patients for its readability and comprehensibility.

\section{Analyses}

The patient-reported data from the questionnaire was linked to the clinical data in the NCR. The following analyses were performed. First, respondent characteristics (age, year of diagnosis, type of surgery, stage of disease, and type of hospital) were compared against non-respondent characteristics to assess generalisability (chi-square, level of significance $p<0.05$ ). Second, patient characteristics and patient-reported health problems plus health care use were presented. Health problems were categorized according to organ system, resulting in eleven categories, and presented against time since diagnosis. Thirdly, the effect of patient and treatment characteristics on the health problems experienced was tested through multivariate logistic regression analyses. A regression was formed for each category of health problems. Levels of significance of 0.10 and 0.05 were applied for univariate and multivariate regression, respectively. Factors tested in relation to reported health problems were: age at time of the survey, time since diagnosis (in years), highest completed level of education, presence of comorbidities, lateralisation of disease, type of surgical intervention (whether or not combined with radiotherapy), axillary surgery, 
chemotherapy (whether or not combined with targeted therapy), anti-hormonal therapy, and breast reconstruction surgery. All analyses were performed in STATA SE14.2 [19].

\section{Results}

Out of 876 invited patients, 404 completed the questionnaire (46\%). Respondents were a representative sample of the invited population based on patient and treatment characteristics, with a slight underrepresentation for the youngest and oldest patients (20\% for respondents versus $25 \%$ for non-respondents for age $<50 ; 17 \%$ for respondents versus $23 \%$ for non-respondents ages $70+; p=0.010$, results not shown). Table 1 shows all patient characteristics. The median age at the time of response was 62.0 years (range: $27.5-91.6)$. One (33\%) or more than one (15\%) comorbid diseases were present in $48 \%$ of patients. Of all patients, $59 \%$ received breast conserving therapy and $41 \%$ received a mastectomy; $21 \%$ also received axillary dissection, and $72 \%$ received treatment with radiotherapy. Adjuvant or neoadjuvant treatment with chemotherapy and anti-hormonal therapy (categories not mutually exclusive) was given to $49 \%$ and $57 \%$ respectively; chemotherapy was combined with trastuzumab in one quarter of patients treated with chemotherapy. These treatment characteristics are representative for the surgically treated breast cancer population in the Netherlands.

Table 1: respondent characteristics $(n=404)$

\begin{tabular}{|c|c|c|}
\hline & $\mathrm{N}(404)$ & $\%$ \\
\hline \multicolumn{3}{|c|}{ Patient characteristics } \\
\hline \multicolumn{3}{|l|}{ Age at time of survey } \\
\hline $\begin{array}{l}\text { Mean } \\
\text { (SD, range) }\end{array}$ & \multicolumn{2}{|c|}{$\begin{array}{c}62.0 \\
(10.9,27.5-91.6\end{array}$} \\
\hline$<50$ & 56 & 14 \\
\hline $50-59$ & 108 & 27 \\
\hline $60-69$ & 133 & 33 \\
\hline $70+$ & 107 & 26 \\
\hline \multicolumn{3}{|l|}{ Time between diagnosis and survey } \\
\hline$<2$ & 83 & 21 \\
\hline $2-3$ & 92 & 23 \\
\hline $3-4$ & 85 & 21 \\
\hline $4-5$ & 89 & 22 \\
\hline$>5$ & 55 & 14 \\
\hline \multicolumn{3}{|l|}{ Highest completed level of education ${ }^{x * *}$} \\
\hline Secondary education or lower & 122 & 30 \\
\hline Medium vocational training (MBO) & 170 & 42 \\
\hline Higher vocational training ( $\mathrm{HBO} /$ university) & 108 & 27 \\
\hline \multicolumn{3}{|l|}{ Comorbidity ${ }^{x * *}$} \\
\hline None & 188 & 47 \\
\hline One & 131 & 33 \\
\hline Two or more & 61 & 15 \\
\hline
\end{tabular}

Table 1 continues on next page 


\begin{tabular}{|c|c|c|}
\hline \multicolumn{3}{|c|}{ Tumour characteristics } \\
\hline \multicolumn{3}{|l|}{ Year of diagnosis } \\
\hline 2012 & 54 & 13 \\
\hline 2013 & 92 & 23 \\
\hline 2014 & 86 & 21 \\
\hline 2015 & 89 & 22 \\
\hline 2016 & 83 & 21 \\
\hline \multicolumn{3}{|l|}{ Stage } \\
\hline 1 & 186 & 46 \\
\hline II & 174 & 43 \\
\hline III & 44 & 11 \\
\hline \multicolumn{3}{|l|}{ Hormone receptor status ${ }^{x}$} \\
\hline HR-positive & 287 & 71 \\
\hline ER or PR positive & 53 & 13 \\
\hline HR-negative & 62 & 15 \\
\hline \multicolumn{3}{|l|}{ Tumour grade ${ }^{x}$} \\
\hline 1 & 95 & 24 \\
\hline 2 & 176 & 44 \\
\hline 3 & 95 & 24 \\
\hline \multicolumn{3}{|c|}{ Treatment characteristics } \\
\hline \multicolumn{3}{|l|}{ Surgery ${ }^{x}$} \\
\hline Breast conserving surgery & 238 & 59 \\
\hline Mastectomy & 160 & 41 \\
\hline \multicolumn{3}{|l|}{ Local treatment modalities: } \\
\hline Axillary dissection & 85 & 21 \\
\hline Radiotherapy & 291 & 72 \\
\hline Immediate breast reconstruction & 36 & 9 \\
\hline \multicolumn{3}{|l|}{ Systemic treatment modalities: } \\
\hline Chemotherapy & 196 & 49 \\
\hline Chemotherapy - with trastuzumab & 50 & 12 \\
\hline Anti-hormonal therapy & 232 & 57 \\
\hline \multicolumn{3}{|c|}{ Hospital characteristics } \\
\hline \multicolumn{3}{|l|}{ Hospital type ${ }^{a}$} \\
\hline General hospital & 166 & 41 \\
\hline Teaching/ academic hospital & 238 & 59 \\
\hline \multicolumn{3}{|l|}{ Hospital volume $^{b}$} \\
\hline Low & 157 & 39 \\
\hline Medium & 88 & 22 \\
\hline High & 159 & 39 \\
\hline
\end{tabular}


Table 2: Health problems categorised by organ system

\begin{tabular}{ll}
\hline Category: & Health problems: \\
\hline Fatigue/stamina & Fatigue/stamina. \\
\hline Cardiac & Palpitations, chest pain or tightness. \\
\hline Respiratory & Cough, complaints in the nose, shortness of breath. \\
\hline Gastrointestinal & Dry mouth, diarrhoea/constipation, gastric or abdominal complaints, nausea. \\
\hline Renal and urinary & Urinary complaints. \\
\hline Central nervous system & $\begin{array}{l}\text { Memory/concentration, tingling hands/feet (neuralgia), irritation of the eyes, } \\
\text { dizziness/vertigo, headache, earache or ear complaints, hypersensitivity to light or } \\
\text { sound. }\end{array}$ \\
\hline Skin & Hair loss, skin problems. \\
\hline Rsychological & $\begin{array}{l}\text { Insomnia, agitation/irritability, anxiety, depressive feelings, sudden feelings of stress } \\
\text { or crisis, increased use of drugs/alcohol. }\end{array}$ \\
\hline Breast & $\begin{array}{l}\text { Menopausal complaints, weight increase/decrease, problems with sex or sexuality, } \\
\text { infertility. }\end{array}$ \\
\hline Musculoskeletal & $\begin{array}{l}\text { Hypersensitivity in the area breast, pain/swelling scars in the breast area, axillary } \\
\text { complaints (including lymphoedema), skin problems in the breast area. }\end{array}$ \\
\hline $\begin{array}{l}\text { Pain/complaints in the upper extremities, pain/complaints in the lower extremities, } \\
\text { neck or shoulder pain/complaints, myalgia/muscle strain, back pain/complaints, } \\
\text { movement restrictions in the arm, fractures. }\end{array}$
\end{tabular}

\section{Health problems}

Health problems were grouped in eleven categories based on organ system (Table 2). In total, 377 out of 404 (93\%; Figure 1) respondents experienced one or more health problems in at least one of these categories. Patients most often reported complaints in the following categories: musculoskeletal health problems (70.5\%); central nervous system health problems $(66.1 \%)$, in particular memory and concentration impairment (42.6\%); fatigue (63.4\%), reproductive system health problems (54.5\%), problems related to the breast $(54.0 \%)$ and psychological health problems (53.0\%; Figure 1).

When stratified by time since diagnosis, the majority of health problems were present to the same extent over time (Table 3). Only the number of patients with fatigue (78\%-56\%, $p=0.033$ ), hair loss $(34 \%-15 \%, p=0.023)$ and premature menopause $(53 \%-35 \%, p=0.024)$ decreased significantly over time, whereas the number of patients with pain and/or complaints in the back (19\%-44\%, $\mathrm{p}=0.022)$ and pain and/or complaints in the lower extremities $(27 \%-51 \%, p=0.023)$ increased significantly over time. 
* Musculoskeletal health problems: Fracture Movement restrictions arm Back pain/complaints Myalgia/ muscle strain

Neck or shoulder pain/complaints Pain/complaints lower extremities: Pain/complaints upper extremities

$$
\text { * Problems breast: }
$$

kin problems breast are Complaints axilla (incl. lymph edema)

Pain/swelling scars breast area Hypersensitivity area breas

${ }^{\star}$ Reproductive system health problems: Problems sex(uality) Weight increase/decrease Menopausal complaints

* Psychological health problems: Increased in drugs/alcohol use Sudden feelings of stress or crisis Depressive feelings Anxiety
Agitation/irritability Insomnia

* Skin health problems: Skin problem Hair loss

* Central nervous system health problems: Hypersensitivity for light or sound Ear pain or complaints Headache Dizziness/vertigo Irritation to the eye(s) hands/feet (neuralgia) Memory/concentration

* Renal and urinary health problems: Urinary complaints

* Gastrointestinal health problems: Gastric or abdominal Nause Diarrhea/constipaints Dry mouth

* Respiratory health problems: Shortness of breath Complaints nose Cough
.

* Cardiac health problems: Chest pain or pressure Palpitations
Pal pressure

* Fatigue/endurance: Fatigue/endurance Total 93.3 $-100 \%$
Reported health problems

Reported health care use

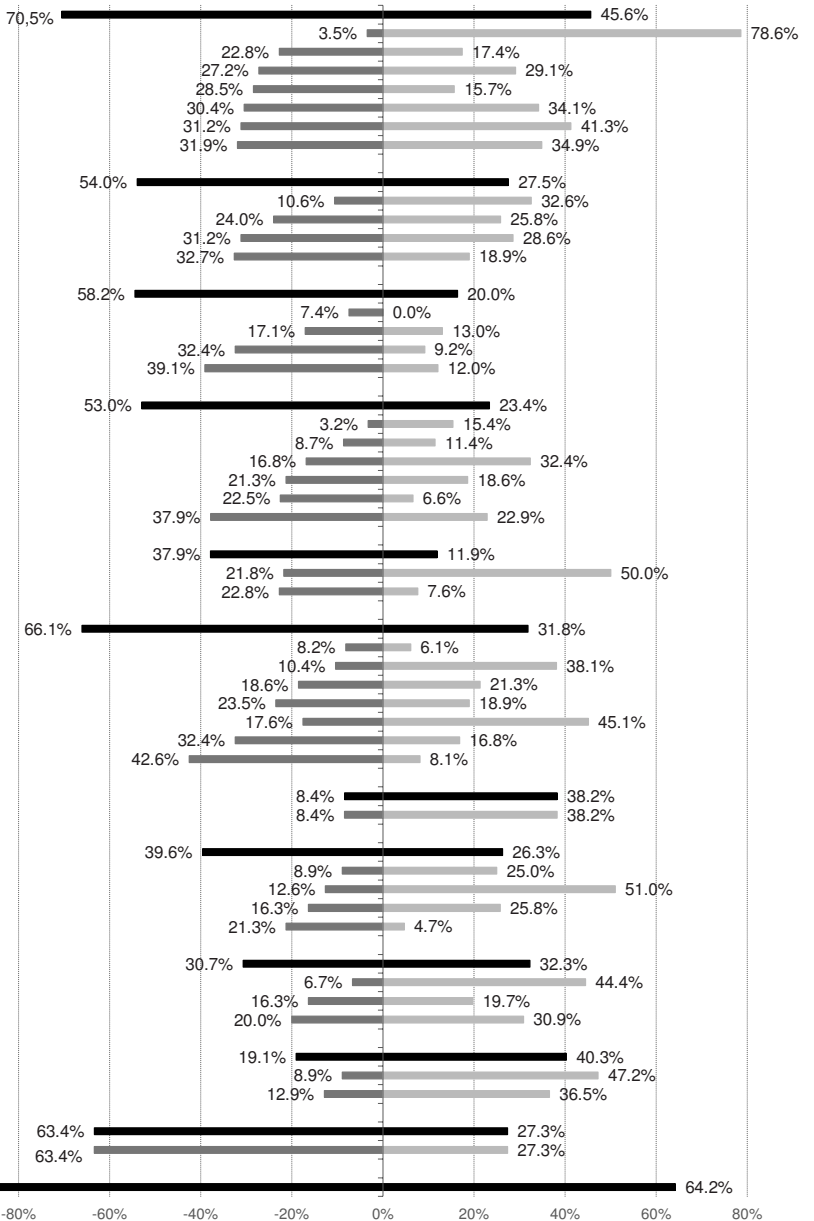

$100 \%$

Figure 1: patient-reported health problems and use of care 


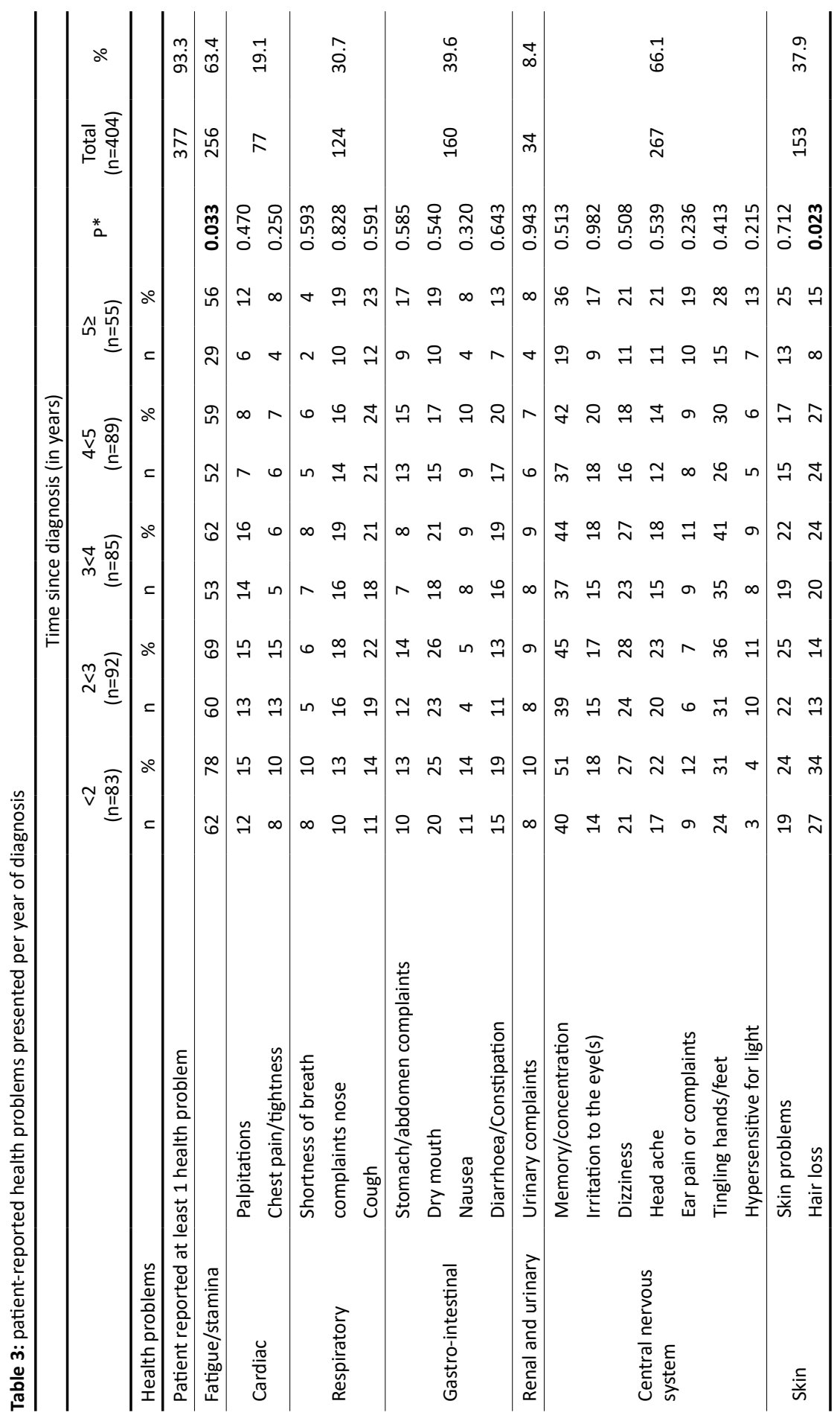




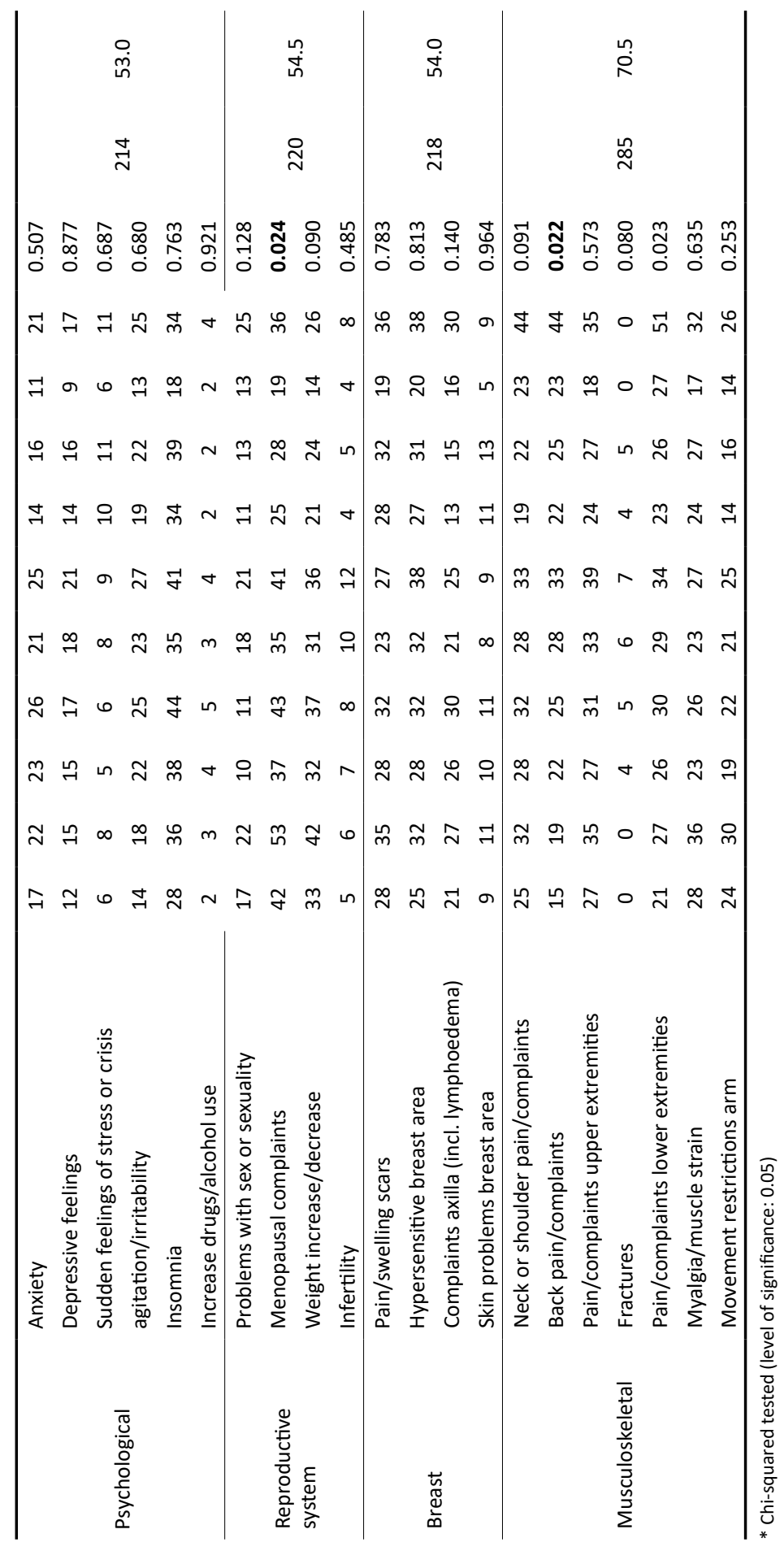




\section{Effect of patient and treatment characteristics on experiencing health problems}

Surgical treatment with adjuvant radiotherapy was provided in $58 \%$ and $14 \%$ of patients with breast conserving surgery and amputation respectively; $25 \%$ had amputations without radiotherapy. At the time of the survey, $39 \%$ of patients were still being treated with anti-hormonal therapy, $11 \%$ had finished treatment ( $7 \%$ unknown $(n=28)$ ). Chemotherapy was administered in $50 \%$ of patients, of which one quarter received additional trastuzumab.

Treatment modalities that were significantly associated with reported health problems based on the multivariate analyses are shown in Table 4. Surgery with adjuvant radiotherapy treatment and axillary dissection were significantly associated with a higher risk of problems in the breast area (breast conserving surgery: OR:3.16, $p<0.001$; amputation: OR:2.25, $p=0.032$; axillary dissection: OR:2.84, $\mathrm{p}=0.001)$. Immediate breast reconstruction was associated with musculoskeletal health problems (OR:4.44, $p=0.022$ ). Treatment with chemotherapy was significantly associated with an increased risk for fatigue (OR: 2.00, $p=0.020$, with trastuzumab OR: $2.40, p=0.031$ ), respiratory health problems (OR:1.81, $p=0.029$; with trastuzumab OR:2.74, $p=0.005)$, gastrointestinal health problems (OR:1.87, $p=0.011$; with trastuzumab OR:2.06, $p=0.035$ ), central nervous system health problems (OR:3.40, $\mathrm{p}<0.001$; with trastuzumab OR:2.51, $\mathrm{p}=0.029$ ), and skin health problems (OR:2.62, $p<0.001$; with trastuzumab OR:2.51, $p=0.029$ ). Currently receiving anti-hormonal therapy was significantly associated with an increased risk of fatigue (OR:1.73, $p=0.040)$, and reproductive system health problems (OR:1.91, $p=0.019)$. Ongoing administration of anti-hormonal therapy was significantly associated with a reduction in central nervous system health problems (OR:0.42, $\mathrm{p}=0.028$ ).

Supplementary Table 1 shows factors that were significant in univariate and multivariate testing. The area under the ROC curve for all eleven regression models ranged between 0.6438 (for skin problems) and 0.7791 (for reproductive system health problems).

\section{Use of care}

Of the $93 \%$ percent of respondents who experienced health problems, $64 \%$ used care for at least one of those problems (Figure 1). Patients most often visited their physician for fractures $(78.6 \%)$, gastric/abdominal complaints (51.0\%), and skin problems $(50.0 \%)$, and least often for infertility $(0.0 \%)$, dry mouth $(4.7 \%)$, and hypersensitivity to light or sound $(6.1 \%)$. The most commonly reported health problems such as fatigue (63.4\%), memory/concentration problems (42.6\%), and menopausal complaints (39.1\%) were not necessarily the ones for which the most use of care was reported $(27.3 \%, 8.1 \%$, and $12.0 \%$ respectively). 


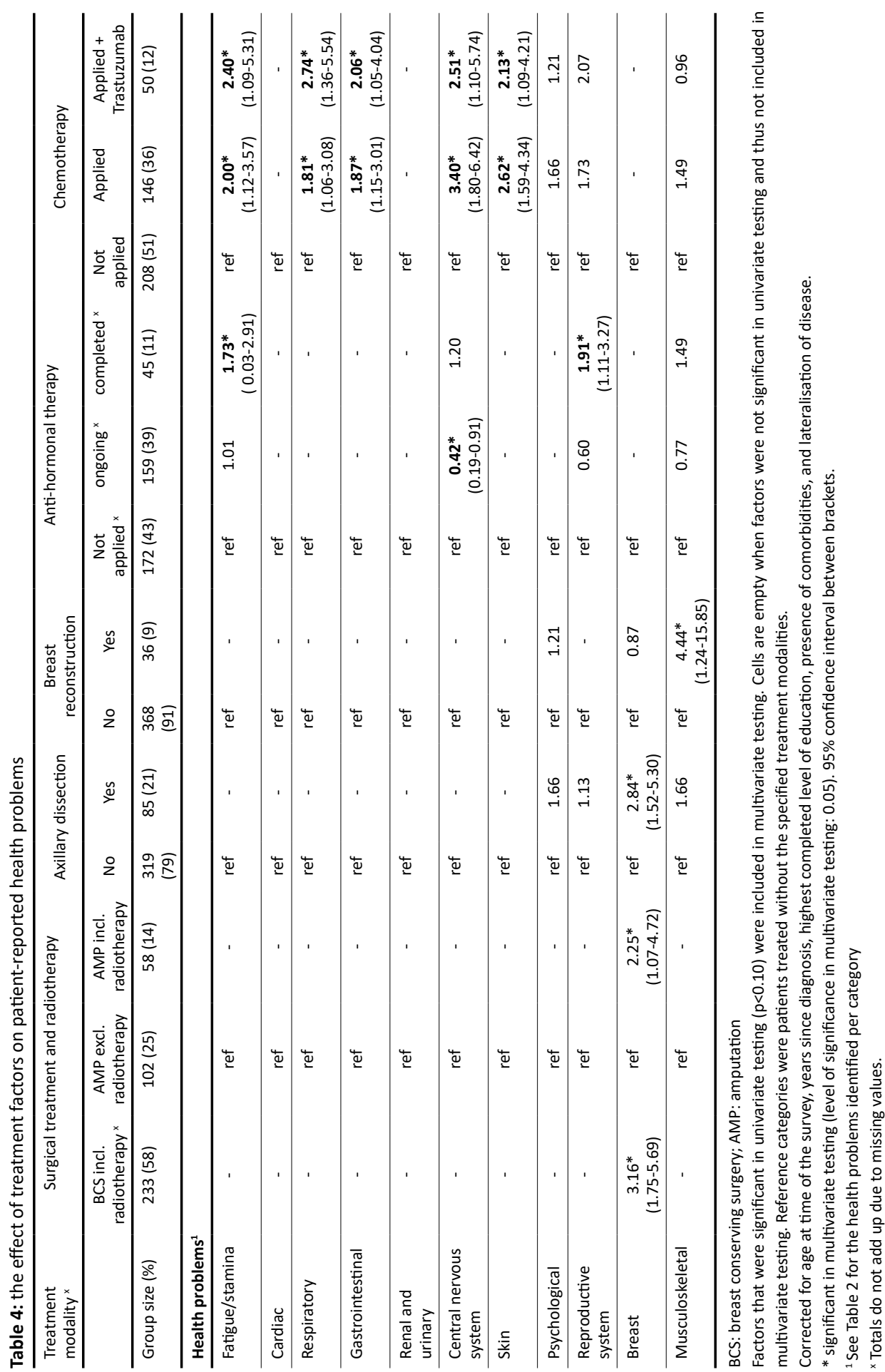




\section{Discussion}

In this study, which uses a survey from 404 women previously treated for early-stage breast cancer, $93 \%$ of respondents experienced one or more health problems during the five-year period after diagnosis. The most frequently reported categories of health problem (by $>50 \%$ of respondents) were: musculoskeletal or related to the nervous system, fatigue, reproductive system, breast area, and psychological health. The majority of health problems were reported consistently over time. The present study found that treatment with chemotherapy and anti-hormonal therapy in particular were associated with a range of health problems. A secondary aim of this study was to identify use of care for the health problems reported. The three most commonly reported health problems for which care was used were fractures, gastric/abdominal complaints, and skin problems. Care was used least for infertility, dry mouth, and hypersensitivity to light or sound.

The results of the present study fit with the literature about treatment-induced effects in breast cancer patients. In multivariate regression analyses, treatment with chemotherapy and antihormonal therapy in particular were associated with an increase in several health problems. The literature has already described a wide range of complaints resulting from chemotherapy, including premature menopause, pneumonitis, bone loss, and skin changes, cardiotoxic effects, neuropathy, and cognitive decline [4-6]. The present study found a positive association between receiving anti-hormonal therapy and reproductive system health problems. Literature states that anti-hormonal treatment may lead to vasomotor symptoms, sexual dysfunction, and infertility [4]. In total, $17 \%$ of our respondents reported problems with sexuality. The actual proportion of patients with sexuality problems may be higher, as patients may feel uncomfortable reporting these problems [20]. Furthermore, the negative effects of autologous breast reconstruction on arm symptoms, and breast conserving therapy (with additional radiotherapy) on breast symptoms have already been described in the literature [21]. The present study found an association between breast reconstruction and musculoskeletal problems (including arm symptoms), and between radiotherapy treatment and health problems in the breast (including skin problems). However, in the utilised database, only data about immediate breast reconstruction was available in the database used, whereas patients treated with radiotherapy may have received delayed breast reconstruction to avoid these effects [22].

Almost all health problems were reported consistently over the time since diagnosis (except for fatigue, hair loss and menopausal complaints), suggesting the majority of problems remained present over time. This was confirmed in a cohort study by Van Leeuwen et al. [2]. The decrease in menopausal complaints may follow from decreasing therapy adherence to anti-hormonal treatment [23]; Hershman et al. [24] stated that $18 \%$ of patients are non-compliant in the first two years. This underlines the need for longitudinal studies to gain an understanding of the possible ongoing cancer-related late effects, that may not have the same development over time in patients. This is important, because persistent symptoms or side-effects have been associated with distress and poor quality of life for breast cancer survivors and cancer survivors in general $[9,13]$. 
This negative effect of continuing symptoms on the quality of life is important regarding the use of care that was evaluated in the present study, which also sheds light on the share of health problems that is presented to the physician: almost all breast cancer patients experienced health problems, but only half of the respondents reported the use of care for one or more of these. Lubberding et al. [15] described how post-treatment symptoms often remain unknown to health care providers, for both social (not wanting to bother people in their surroundings) and practical reasons (short consultation time, not having a follow-up appointment when symptoms emerged, not knowing who to contact in the event of symptoms). However, they noted as well that a complete overview of the patient's health status was missing, resulting in a lack of coordination of care and suboptimal referral to supporting care services. However, a complete overview of health status should be the foundation of follow-up for later effects of breast cancer treatment. During consultations, this overview could be obtained by administering and discussing of patient-reported outcome measures that consist of symptom-specific measurement scales addressing both physical and psychosocial topics $[25,26]$, or by symptom checklists $[27,28]$. Long-term regular assessment of these measures during follow-up means that health problems that persist become a burden later are detected as well.

Discrepancies with the literature were found as well. No treatment factors were associated with the risk of cardiac health problems, even though the risk of cardiovascular disease is increased by $30 \%$ in cancer survivors and was significantly associated with radiotherapy and systemic treatment $[7,8,29]$. The increased risks for coronary events starts within the five years after radiotherapy, but may continue for up to 20-30 years [8,29]. Effects that develop over a period of time longer than five years, may therefore have been missed in the present study. Furthermore, no significant association between chemotherapy and reproductive system health problems was found, whereas literature reports infertility in more than half of patients younger than 40 [4]. However, only $4 \%$ of respondents $(n=16)$ were younger than 40 , possibly reducing the power to detect this effect.

Some notes are needed when interpreting the results of this study. The application of patientreported surveys is related to forms of bias, including recall bias and response bias. Recall bias might lead to underestimation of complaints, as patients may not recall all the health problems they experienced. Response bias may lead to underestimation or overestimation, as patients with poorer health may either feel more urgency or feel too sick to participate. Underestimation of health problems may have occurred, as $79 \%$ of patients with fractures reported use of the healthcare system, where this would be expected to be $100 \%$. The interpretation of our results could be improved by comparing them against health problems in the general population, to better understand which health problems are more prevalent in breast cancer patients. This step will be undertaken in our further research. Most breast cancer patients $(76 \%)$ in a study by Hagen et al. [30] reported health complaints equally as often as the controls, however, health problems such as leg pain, interrupted sleep, hot flushes, tiredness, dizziness, and diarrhoea were reported significantly more by patients than controls. Lastly, interaction between treatment modalities that were associated with the health problems reported were tested, but no interactions were found. Friese et al. [31] found that receiving combined chemotherapy and radiation therapy was associated with a greater severity of toxicity reported. 


\section{Implications for practice}

Breast cancer is the most commonly diagnosed cancer in women worldwide, estimated at 2.1 million new diagnoses in 2018 [32]. Breast cancer patients in North America and Europe have a five-year survival of over $80 \%$ [33]. The extrapolation of the health problems found in the present study to this enormous group of surviving patients endorses the importance of proper follow-up care. The factors associated with the health problems found in this study could be used in multiple ways. First, they could be helpful in recognising current symptoms in relation to previous cancer treatment [9]. This could also be important for general practitioners, who might be consulted by patients about health issues induced by breast cancer treatment long after follow-up is completed [9]. Secondly, it would be applicable in a prospective model of monitoring, to promote monitoring and healthy behaviours, to provide education about health problems and early identification of them, and to introduce rehabilitation when limitations are identified [34]. Thirdly, information about health problems after breast cancer treatment focus more on specific target groups, ranging from patients with low to high burdens of symptoms in terms of pain, psychological complaints and fatigue, and burdens due to other complaints [35]. Patient-reported outcome measures (PROMS), for instance the standard set suggested by the International Consortium for Health Outcomes Measurement (ICHOM) Initiative, can be used to monitor health problems and quality of life [36].

\section{Conclusion}

Almost all patients treated for early-stage breast cancer experienced health problems up to five years after diagnosis. The most frequent categories of health problems $(>50 \%)$ were related to the musculoskeletal system, nervous system, fatigue, reproductive system, breast area, and psychological health. Treatment with chemotherapy and anti-hormonal therapy in particular were associated with health problems being reported. Factors associated with the development of health problems are useful for informing patients better beforehand and for targeting followup care. 


\section{References}

1. Kenyon M, Mayer DK, Owens AK. Late and Long-Term Effects of Breast Cancer Treatment and Surveillance Management for the General Practitioner. Journal of Obstetric, Gynecologic \& Neonatal Nursing. 2014;43:382-98.

2. van Leeuwen M , Husson O, Alberti P, Arraras JI, Chinot OL, Costantini A, et al. Understanding the quality of life (QOL) issues in survivors of cancer: towards the development of an EORTC QOL cancer survivorship questionnaire. Health and Quality of Life Outcomes. 2018;16(1).

3. Binkley JM, Harris SR, Levangie PK, Pearl M, Guglielmino J, Kraus V, et al. Patient Perspectives on Breast Cancer Treatment Side Effects and the Prospective Surveillance Model for Physical Rehabilitation for Women With Breast Cancer. Cancer. 2012;118:2207-16.

4. Ewertz $M$, Jensen $A B$. Late effects of breast cancer treatment and potentials for rehabilitation. Acta Oncologica. 2011;50:187-93.

5. Agrawal S. Late effects of cancer treatment in breast cancer survivors. South Asian Journal of cancer. 2014;3(2).

6. Pinto AC, de Azambujab E. Improving quality of life after breast cancer: Dealing with symptoms. Maturitas. 2011;70:343-8.

7. Eschenhagen T, Force T, Ewer MS, de Keulenaer GW, Suter TM, Anker SD, et al. Cardiovascular side effects of cancer therapies: a position statement fromthe Heart Failure Association of the European Society of Cardiology. European Journal of Heart Failure. 2011;13:1-10.

8. Maas AHEM, Ottevanger N, Atsma F, MJ C, Leinere T, Poortmans P. Cardiovascular surveillance in breast cancer treatment: A more individualized approach is needed. Maturitas. 2016;89:58-62.

9. Mayer DK, Nasso SF, Earp JA. Defining cancer survivors, their needs, and perspectives on survivorship health care in the USA. The Lancet. 2017;18(1):e11-e8.

10. Netherlands Comprehensive Cancer Organisation (IKNL). National guideline on Breast Cancer. Netherlands Comprehensive Cancer Organisation (IKNL),,; 2012.

11. Runowicz CD, Leach CR, Henry NL, Henry KS, Mackey HT, Cowens-Alvarado RL, et al. American Cancer Society/American Society of Clinical Oncology Breast Cancer Survivorship Care Guideline. Journal of Clinical Oncology. 2016;34(6):611-35.

12. Senkus E, Kyriakides S, Ohno S, Penault-Llorca F, Poortmans P, Rutgers E, et al. Primary breast cancer: ESMO Clinical Practice Guidelines for diagnosis, treatment and follow-up. Annals of Oncology. 2015;26:8-30.

13. Rosedale M, Fu MR. Confronting the Unexpected: Temporal, Situational, and Attributive Dimensions of Distressing Symptom Experience for Breast Cancer Survivors. Oncology Nursing Forum. 2010;37(1).

14. Chawla N, Blanch-Hartigan D, Virgo KS, Ekwueme DU, Han X, Forsythe L, et al. Quality of Patient-Provider Communication Among Cancer Survivors: Findings From a Nationally Representative Sample. Journal of Oncology Practise. 2016;12(12).

15. Lubberding S, van Uden-Kraan CF, Te Velde EA, Cuijpers P, Leemans R, Verdonck-de Leeuw IM. Improving access to supportive cancer care through an eHealth application: a qualitative needs assessment among cancer survivors. Journal of Clinical Nursing. 2015;24:1367-79.

16. Netherlands Comprehensive Cancer Organisation (IKNL). About the registration 2018 [Available from: https://www.cijfersoverkanker.nl/about-the-registration-37.html.

17. van de Poll-Franse LV, Horevoorts N, Eenbergen M, Denollet J, Roukema JA, Aaronson NK, et al. The Patient Reported Outcomes Following Initial treatment and Long term Evaluation of Survivorship registry: Scope, rationale and design of an infrastructure for the study of physical and psychosocial outcomes in cancer survivorship cohorts. European journal of cancer (Oxford, England : 1990). 2011;47(14):2188-94.

18. Yzermans J, Baliatsas C, van Dulmen S, van Kamp I. Assessing non-specific symptoms in epidemiological studies: development and validation of the symptoms and perceptions (SaP) questionnaire. International journal of hygiene and environmental health. 2016;219(1):53-65.

19. StataCorp. Stata Statistical Software: Release 14. In: College Station TSL, ,, editor. 2015.

20. Stabile C, Goldfarb S, Baser RE, Goldfrank DJ, Abu-Rustum NR, Barakat RR, et al. Sexual Health Needs and Educational Intervention Preferences for Women with Cancer. Breast Cancer Res Treat. 2017;165(1):77-84. 
21. Lagendijk M, van Egdom LSE, Richel C, van Leeuwen N, Verhoef C, Lingsma HF, et al. Patient reported outcome measures in breast cancer patients. European Journal of Surgical Oncology. 2018;44:963-8.

22. Mureau MAM, Nederlandse Vereniging voor Plastische Chirurgie (NVPC). Dutch Breast Reconstruction Guideline. Journal of Plastic Reconstructive and Aesthetic Surgery 2018:290-304.

23. Bluethmann SM, Murphy CC, Tiro JA, Mollica MA, Vernon SW, Bartholomew LK. Deconstructing Decisions to Initiate, Maintain, or Discontinue Adjuvant Endocrine Therapy in Breast Cancer Survivors: A Mixed-Methods Study. Oncol Nurs Forum. 2017;44(3):E101-e10.

24. Hershman DL, Kushi LH, Hillyer GC, Coromilas E, Buono D, Lamerato L, et al. Psychosocial factors related to non-persistence with adjuvant endocrine therapy among women with breast cancer: the Breast Cancer Quality of Care Study (BQUAL). Breast Cancer Res Treat. 2016;157(1):133-43.

25. Wintner LM, Sztankay M, Aaronson N, Bottomley A, Giesinger JM, Groenvold M, et al. The use of EORTC measures in daily clinical practiced A synopsis of a newly developed manual. European Journal of Cancer. 2016;68:73-81.

26. Cano SJ, Klassen AF, Scott AM, Pusic AL. A Closer Look at the BREAST-Q. Clin Plastic Surg. 2013;40:287-96.

27. Netherlands Comprehensive Cancer Organisation (IKNL). Guideline on detecting psychosocial care needs in cancer patients. 2017.

28. Williams PD, Graham KM, Storlie DL, Pedace TM, Haeflinger KV, Williams DD, et al. Therapy-related symptom checklist use during treatments at a cancer center. Cancer Nurs. 2013;36(3):245-54.

29. Naaktgeboren WR, Linschoten M, de Graeff A, van Rhenen A, Cramer MJ, Asselbergs FW, et al. Long-term cardiovascular health in adult cancer survivors. Maturitas. 2017;105:37-45.

30. Hagen KB, Aas T, Kvaløy JT, Eriksen HR, Søiland H, Lind R. Fatigue, anxiety and depression overrule the role of oncological treatment in predicting self-reported health complaints in women with breast cancer compared to healthy controls. The Breast. 2016;28:100-6.

31. Friese CR, Harrison JM, Janz NK, Jagsi R, Morrow M, Yun Li Y, et al. Treatment-Associated Toxicities Reported by Patients with Early-Stage Invasive Breast Cancer. Cancer. 2017;123(11):1925-34.

32. Bray F, Ferlay J, Soerjomataram I, Siegel RL, Torre LA, Jemal A. Global Cancer Statistics 2018: GLOBOCAN Estimates of Incidence and Mortality Worldwide for 36 Cancers in 185 Countries. CA: A Cancer Journal for Clinicians. 2018;0:1-31.

33. Coleman MP, Quaresma M, Berrino F, Lutz JM, De Angelis R, Capocaccia R, et al. Cancer survival in five continents: a worldwide population-based study (CONCORD). Lancet Oncology. 2008;9:730-56.

34. Stout NL, Binkley JM, Schmitz KH, Andrews K, Hayes SC, Campbell KL, et al. A Prospective Surveillance Model for Rehabilitation for Women With Breast Cancer. Cancer. 2012;118(8):2191-200.

35. Avis NE, Levine B, Marshall SA, Ip EH. Longitudinal Examination of Symptom Profiles Among Breast Cancer Survivors. Journal of Pain and Symptom Management. 2017;53(4):703-10.

36. Ong WL, Schouwenburg MG, van Bommel ACM, Stowell C, Allison KH, Benn KE, et al. A Standard Set of ValueBased Patient-Centered Outcomes for Breast Cancer: The International Consortium for Health Outcomes Measurement (ICHOM) Initiative. JAMA Oncology. 2017;3(5):677-85. 
Appendix 1: Survey

0. What is your date of birth?

..-.-19..

1. In general, would you say your health is:

1 - Excellent

2 - Very good

3 - Good

4 - Fair

5 - Poor

2. I seem to get sick a little more easily than other people

Definitely true - Mostly true - Don't know - Mostly false - Definitely false

3. I am as healthy as anybody I know

Definitely true - Mostly true - Don't know - Mostly false - Definitely false

4. I expect my health to get worse

Definitely true - Mostly true - Don't know - Mostly false - Definitely false

5. My health is excellent

Definitely true - Mostly true - Don't know - Mostly false - Definitely false

6. Compared to one year ago, how would you rate your health in general now?

1 - Much better now than one year ago

2 - Somewhat better now than one year ago

3 - About the same

4 - Somewhat worse now than one year ago

5 - Much worse now than one year ago

7. Do you currently suffer from one or more of the diseases or conditions listed below?

Any other type of cancer - pulmonary disease - cardiovascular disease - gastrointestinal disease - disease of the urinary or reproductive system - musculoskeletal disease - central nervous system metabolic disorder or coagulopathy - infectious disease - none - other

In case you do: for which of these did you receive treatment?

8. Which of the following health problems have you experienced over the recent year? And for which of these health problems did you visit a primary care physician or other doctor?

Palpitations

Chest pain/tightness

Shortness of breath

Nasal complaints

Cough

Stomach/abdomen complaints

Dry mouth

Nausea

Diarrhoea/constipation

Urinary complaints

Memory/concentration

Irritation to the eye(s) 
Dizziness

Head ache

Earache or complaints

Tingling hands/feet

Hypersensitivity to light

Skin problems

Hair loss

Anxiety

Depressive feelings

Sudden feelings of stress or crisis

Agitation/irritability

Insomnia

Increased use of drugs or alcohol

Problems with sex or sexuality

Menopausal complaints

Weight increase/decrease

Infertility

Pain/swelling of scars

Hypersensitivity in the breast area

Complaints axilla (incl. lymphoedema)

Skin problems in the breast area

Neck or shoulder pain/complaints

Back pain/complaints

Pain/complaints of the upper extremities

Fractures

Pain/complaints of the lower extremities

Myalgia/muscle strain

Movement restrictions of the arm

9. What was your key health problem in the list above? Fill in only one health problem.

(...)

13. What is your highest completed level of education? (i.e. with a diploma or certificate)

No education - lower education - middle education - higher education - other

14. Do you have a partner?

Yes - No

15. Do you have children?

Yes, they live with me - Yes, they live on their own - No

16. What description is most applicable to you at this moment? (please tick one answer)

Attending school/education - paid employment - unemployed/seeking work - incapacitated homemaker - retirement

17. Are you currently under treatment for your breast cancer?

No, I have finished my treatment - Yes, I am currently receiving anti-hormonal treatment - Yes, I am receiving [fill in the treatment you are currently receiving] 
Supplementary Table 1: the effect of patient and treatment factors on health problems experienced

\begin{tabular}{|c|c|c|c|c|c|c|c|}
\hline & & \multirow{2}{*}{$\begin{array}{c}\text { Total } \\
\text { respondents }\end{array}$} & \multicolumn{2}{|c|}{ Univariate } & \multicolumn{2}{|c|}{ Multivariate } & \multirow[t]{2}{*}{$\mathrm{P}^{*}$} \\
\hline & & & OR & $95 \% \mathrm{Cl}$ & OR & $95 \% \mathrm{Cl}$ & \\
\hline \multicolumn{8}{|c|}{ Fatigue/stamina ( $n=256 / 404$ patients experienced problems) } \\
\hline \multirow{5}{*}{$\begin{array}{l}\text { Time since diagnosis } \\
\text { (years) }\end{array}$} & $<2$ & 79 & ref & & ref & & \\
\hline & $2-3$ & 87 & 0.61 & $0.30-1.23$ & 0.59 & $0.28-1.24$ & 0.163 \\
\hline & $3-4$ & 85 & 0.45 & $0.23-0.91$ & 0.45 & $0.21-0.97$ & 0.041 \\
\hline & $4-5$ & 88 & 0.40 & $0.20-0.79$ & 0.37 & $0.18-0.77$ & 0.008 \\
\hline & $>5$ & 52 & 0.35 & $0.16-0.74$ & 0.27 & $0.12-0.64$ & 0.003 \\
\hline \multirow{2}{*}{ Comorbidities** } & No & 185 & ref & & ref & & \\
\hline & Yes & 188 & 1.82 & $1.18-2.80$ & 2.23 & $1.37-3.63$ & 0.001 \\
\hline \multirow{3}{*}{ Chemotherapy } & No & 208 & ref & & ref & & \\
\hline & Chemotherapy & 146 & 2.27 & $1.42-3.63$ & 2.00 & $1.12-3.57$ & 0.020 \\
\hline & $\begin{array}{l}\text { Chemotherapy } \\
\text { with trastuzumab }\end{array}$ & 50 & 2.37 & $1.17-4.82$ & 2.40 & $1.09-5.31$ & 0.031 \\
\hline \multirow{3}{*}{ Anti-hormonal therapy $X$} & No & 132 & ref & & ref & & \\
\hline & Yes, completed & 44 & 1.24 & $0.62-2.48$ & 1.01 & $0.48-2.12$ & 0.976 \\
\hline & Yes, still receiving & 155 & 2.39 & $1.46-3.92$ & 1.73 & $1.03-2.91$ & 0.040 \\
\hline \multicolumn{8}{|c|}{ Area under the ROC-curve: 0.6922} \\
\hline \multicolumn{8}{|c|}{ Cardiac health problems ( $n=77 / 404$ patients experienced problems) } \\
\hline n/a & $\mathrm{n} / \mathrm{a}$ & $\mathrm{n} / \mathrm{a}$ & $\mathrm{n} / \mathrm{a}$ & $\mathrm{n} / \mathrm{a}$ & $\mathrm{n} / \mathrm{a}$ & $\mathrm{n} / \mathrm{a}$ & $\mathrm{n} / \mathrm{a}$ \\
\hline \multicolumn{8}{|c|}{ Respiratory health problems ( $n=124 / 404$ patients experienced problems) } \\
\hline \multirow{4}{*}{$\begin{array}{l}\text { Age at time of } \\
\text { questionnaire (years) }\end{array}$} & $<50$ & 56 & ref & & ref & & \\
\hline & $50-59$ & 107 & 2.14 & $1.00-4.74$ & 2.41 & $1.05-5.51$ & 0.037 \\
\hline & $60-69$ & 130 & 2.27 & $1.05-4.94$ & 2.64 & $1.17-5.98$ & 0.019 \\
\hline & $70+$ & 98 & 2.79 & $1.26-6.18$ & 3.67 & $1.53-8.79$ & 0.004 \\
\hline \multirow{2}{*}{ Comorbidities** } & No & 186 & Ref & & Ref & & \\
\hline & Yes & 187 & 2.21 & $1.41-3.45$ & 2.25 & $1.41-3.60$ & 0.001 \\
\hline \multirow{3}{*}{ Chemotherapy } & No & 208 & ref & & ref & & \\
\hline & Chemotherapy & 146 & 1.10 & $0.69-1.76$ & 1.81 & $1.06-3.08$ & 0.029 \\
\hline & $\begin{array}{l}\text { Chemotherapy } \\
\text { with trastuzumab }\end{array}$ & 50 & 1.99 & $1.05-3.78$ & 2.74 & $1.36-5.54$ & 0.005 \\
\hline
\end{tabular}

Area under the ROC-curve: 0.6

Supplementary Table 1 continues on next page 
Continuation of supplementary Table 1

\begin{tabular}{|c|c|c|c|c|c|c|c|}
\hline & & \multirow{2}{*}{$\begin{array}{l}\text { Total } \\
\text { respondents }\end{array}$} & \multicolumn{2}{|c|}{ Univariate } & \multicolumn{2}{|c|}{ Multivariate } & \multirow[t]{2}{*}{$\mathrm{P}^{*}$} \\
\hline & & & OR & $95 \% \mathrm{Cl}$ & OR & $95 \% \mathrm{Cl}$ & \\
\hline \multicolumn{8}{|c|}{ Gastrointestinal health problems ( $n=160 / 404$ patients experienced problems) } \\
\hline \multirow{3}{*}{$\begin{array}{l}\text { Highest completed level } \\
\text { of education** }\end{array}$} & $\begin{array}{l}\text { Low level } \\
\text { education }\end{array}$ & 122 & Ref & & ref & - & - \\
\hline & $\begin{array}{l}\text { Middle level } \\
\text { education }\end{array}$ & 168 & 0.62 & $0.38-1.02$ & 0.57 & 0.34-0.97 & 0.037 \\
\hline & $\begin{array}{l}\text { High level } \\
\text { education }\end{array}$ & 106 & 0.92 & $0.54-1.57$ & 0.87 & $0.49-1.53$ & 0.620 \\
\hline \multirow{2}{*}{ Comorbidities** } & No & 184 & Ref & & Ref & & \\
\hline & Yes & 187 & 2.09 & $1.37-3.18$ & 2.21 & $1.42-3.44$ & $<0.001$ \\
\hline \multirow{3}{*}{ Chemotherapy } & No & 208 & ref & & ref & & \\
\hline & Chemotherapy & 146 & 1.36 & $0.87-2.11$ & 1.87 & $1.15-3.01$ & 0.011 \\
\hline & $\begin{array}{l}\text { Chemotherapy } \\
\text { with trastuzumab }\end{array}$ & 50 & 1.98 & $1.05-3.72$ & 2.06 & $1.05-4.04$ & 0.035 \\
\hline \multicolumn{8}{|c|}{ Area under the ROC-curve: 0.6438} \\
\hline \multicolumn{8}{|c|}{ Renal/urinary health problems $(n=34 / 404)$} \\
\hline \multirow{2}{*}{ Comorbidities } & No & 187 & ref & & Ref & & \\
\hline & Yes & 187 & 2.91 & $1.31-6.45$ & 2.73 & $1.19-6.26$ & 0.018 \\
\hline \multirow{2}{*}{ Lateralisation } & left & 197 & Ref & & Ref & & \\
\hline & right & 195 & 0.45 & $0.21-0.96$ & 0.39 & $0.18-0.87$ & 0.021 \\
\hline \multicolumn{8}{|c|}{ Area under the ROC-curve: 0.7424} \\
\hline \multicolumn{8}{|c|}{ Nerve system health problems ( $n=267 / 404$ patients experienced problems) } \\
\hline \multirow{4}{*}{$\begin{array}{l}\text { Age at time of } \\
\text { questionnaire (years) }\end{array}$} & $<50$ & 56 & ref & & ref & & \\
\hline & $50-59$ & 107 & 0.45 & $0.20-1.02$ & 0.54 & $0.22-1.33$ & 0.180 \\
\hline & $60-69$ & 129 & 0.43 & $0.19-0.95$ & 0.58 & $0.24-1.42$ & 0.236 \\
\hline & $70+$ & 98 & 0.25 & $0.11-0.58$ & 0.37 & $0.14-0.98$ & 0.044 \\
\hline \multirow{5}{*}{$\begin{array}{l}\text { Time since diagnosis } \\
\text { (years) }\end{array}$} & $>2$ & 78 & ref & & ref & & \\
\hline & $2-3$ & 87 & 0.55 & $0.28-1.09$ & 0.36 & $0.17-0.78$ & 0.009 \\
\hline & $3-4$ & 85 & 0.92 & $0.45-1.87$ & 0.65 & $0.29-1.46$ & 0.298 \\
\hline & $4-5$ & 88 & 0.56 & $0.29-1.11$ & 0.43 & $0.20-0.93$ & 0.033 \\
\hline & $>5$ & 52 & 0.61 & $0.28-1.31$ & 0.56 & $0.23-1.37$ & 0.204 \\
\hline \multirow{2}{*}{ Comorbidities $* *$} & No & 185 & Ref & & Ref & & \\
\hline & Yes & 187 & 1.85 & $1.19-2.86$ & 2.84 & $1.67-4.80$ & $<0.001$ \\
\hline \multirow{3}{*}{ Chemotherapy } & No & 208 & ref & & ref & & \\
\hline & Chemotherapy & 146 & 3.24 & $1.96-5.37$ & 3.40 & $1.80-6.42$ & $<0.001$ \\
\hline & $\begin{array}{l}\text { Chemotherapy } \\
\text { with trastuzumab }\end{array}$ & 50 & 2.97 & $1.40-6.28$ & 2.51 & $1.10-5.74$ & 0.029 \\
\hline \multirow{3}{*}{ Anti-hormonal therapy $\mathrm{X}$} & No & 132 & ref & & ref & & \\
\hline & Yes, completed & 44 & 0.55 & $0.28-1.10$ & 0.42 & $0.19-0.91$ & 0.028 \\
\hline & Yes, still receiving & 154 & 1.63 & $0.98-2.71$ & 1.20 & $0.69-2.10$ & 0.519 \\
\hline
\end{tabular}




\begin{tabular}{|c|c|c|c|c|c|c|c|}
\hline \multicolumn{8}{|c|}{ Skin problems ( $n=153 / 404$ patients experienced problems) } \\
\hline \multirow{2}{*}{ Comorbidities** } & No & 187 & Ref & & Ref & & \\
\hline & Yes & 187 & 1.53 & $1.01-2.32$ & 1.91 & $1.22-2.99$ & 0.005 \\
\hline \multirow{3}{*}{ Chemotherapy } & No & 208 & ref & & ref & & \\
\hline & Chemotherapy & 146 & 2.41 & $1.54-3.77$ & 2.62 & $1.59-4.34$ & $<0.001$ \\
\hline & $\begin{array}{l}\text { Chemotherapy } \\
\text { with trastuzumab }\end{array}$ & 50 & 2.13 & $1.12-4.03$ & 2.13 & $1.09-4.21$ & 0.028 \\
\hline \multicolumn{8}{|c|}{ Area under the ROC-curve: 0.6614} \\
\hline \multicolumn{8}{|c|}{ Psychological health problems ( $n=214 / 404$ patients experienced problems) } \\
\hline \multirow{4}{*}{$\begin{array}{l}\text { Age at time of } \\
\text { questionnaire (years) }\end{array}$} & $<50$ & 56 & ref & & ref & & \\
\hline & $50-59$ & 107 & 0.73 & $0.37-1.45$ & 0.78 & $0.38-1.61$ & 0.503 \\
\hline & $60-69$ & 129 & 0.57 & $0.30-1.10$ & 0.65 & $0.32-1.32$ & 0.234 \\
\hline & $70+$ & 98 & 0.33 & $0.16-0.65$ & 0.37 & $0.17-0.81$ & 0.012 \\
\hline \multirow{2}{*}{ Comorbidities** } & No & 185 & Ref & & Ref & & \\
\hline & Yes & 187 & 1.43 & $0.95-2.15$ & 1.87 & $1.20-2.90$ & 0.006 \\
\hline \multirow{2}{*}{ Lateralisation } & left & 202 & Ref & & ref & & \\
\hline & right & 202 & 0.66 & $0.44-0.98$ & 0.65 & $0.42-1.00$ & 0.049 \\
\hline \multicolumn{8}{|c|}{ Area under the ROC-curve: 0.6629} \\
\hline \multicolumn{8}{|c|}{ Reproductive system health problems ( $\mathrm{n}=\mathbf{2 2 0 / 4 0 4}$ patients experienced problems) } \\
\hline \multirow{4}{*}{$\begin{array}{l}\text { Age at time of } \\
\text { questionnaire (years) }\end{array}$} & $<50$ & 56 & ref & & ref & & \\
\hline & $50-59$ & 107 & 0.81 & $0.37-1.75$ & 0.89 & $0.39-2.07$ & 0.795 \\
\hline & $60-69$ & 130 & 0.25 & $0.12-0.51$ & 0.24 & $0.11-0.55$ & 0.001 \\
\hline & $70+$ & 99 & 0.14 & $0.07-0.31$ & 0.13 & $0.05-0.38$ & $<0.001$ \\
\hline \multirow{5}{*}{$\begin{array}{l}\text { Time since diagnosis } \\
\text { (years) }\end{array}$} & $>2$ & 79 & ref & & ref & & \\
\hline & $2-3$ & 87 & 0.66 & $0.35-1.24$ & 0.57 & $0.27-1.22$ & 0.148 \\
\hline & $3-4$ & 85 & 0.63 & $0.33-1.20$ & 0.52 & $0.24-1.12$ & 0.095 \\
\hline & $4-5$ & 88 & 0.39 & $0.20-0.73$ & 0.42 & $0.20-0.89$ & 0.024 \\
\hline & $>5$ & 53 & 0.45 & $0.22-0.91$ & 0.43 & $0.18-1.01$ & 0.053 \\
\hline \multirow{2}{*}{ Comorbidities** } & No & 187 & Ref & & Ref & & \\
\hline & Yes & 187 & 1.48 & $0.98-2.24$ & 2.41 & $1.45-4.03$ & 0.001 \\
\hline \multirow{3}{*}{ Anti-hormonal therapy $X$} & No & 133 & ref & & ref & & \\
\hline & Yes, completed** & 44 & 0.85 & $0.43-1.65$ & 0.60 & $0.27-1.33$ & 0.210 \\
\hline & $\begin{array}{l}\text { Yes, still } \\
\text { receiving** }\end{array}$ & 154 & 2.18 & $1.39-3.42$ & 1.91 & $1.11-3.27$ & 0.019 \\
\hline
\end{tabular}

Supplementary Table 1 continues on next page 
Continuation of supplementary Table 1

\begin{tabular}{|c|c|c|c|c|c|c|c|}
\hline & & \multirow{2}{*}{$\begin{array}{c}\text { Total } \\
\text { respondents }\end{array}$} & \multicolumn{2}{|c|}{ Univariate } & \multicolumn{2}{|c|}{ Multivariate } & \multirow[t]{2}{*}{$\mathrm{P}^{*}$} \\
\hline & & & OR & $95 \% \mathrm{Cl}$ & OR & $95 \% \mathrm{Cl}$ & \\
\hline \multicolumn{8}{|c|}{ Problems in breast area ( $n=218 / 404$ patients experienced problems) } \\
\hline \multirow{3}{*}{$\begin{array}{l}\text { Surgery followed by } \\
\text { radiotherapy }\end{array}$} & $\begin{array}{l}\text { Breast conserving } \\
\text { surgery with } \\
\text { radiotherapy }\end{array}$ & 227 & 2.77 & $1.70-4.52$ & 3.16 & $1.75-5.69$ & $<0.001$ \\
\hline & $\begin{array}{l}\text { Amputation } \\
\text { without } \\
\text { radiotherapy }\end{array}$ & 98 & ref & & Ref & & \\
\hline & $\begin{array}{l}\text { Amputation with } \\
\text { radiotherapy }\end{array}$ & 56 & 3.1 & $1.56-6.14$ & 2.25 & $1.07-4.72$ & 0.032 \\
\hline \multirow{2}{*}{ Axillary dissection } & No & 310 & ref & & ref & & \\
\hline & Yes & 82 & 1.97 & $1.17-3.30$ & 2.84 & $1.52-5.30$ & 0.001 \\
\hline \multicolumn{8}{|c|}{ Area under the ROC-curve: 0.6606} \\
\hline \multicolumn{8}{|c|}{ Musculoskeletal health problems ( $n=285 / 404$ patients experienced problems) } \\
\hline \multirow{2}{*}{ Comorbidities** } & No & 186 & Ref & & Ref & & $<0.001$ \\
\hline & Yes & 187 & 2.70 & $1.66-4.41$ & 3.44 & $1.99-5.95$ & \\
\hline \multirow{2}{*}{$\begin{array}{l}\text { Immediate breast } \\
\text { reconstruction }\end{array}$} & No & 356 & ref & & ref & & 0.022 \\
\hline & Yes & 35 & 2.38 & $0.90-6.30$ & 4.44 & $\begin{array}{l}1.24- \\
15.85\end{array}$ & \\
\hline
\end{tabular}

Only factors that were significant in the multivariate testing were included in this table. Factors tested in univariate regression were: age at time of survey, years since diagnosis, highest completed level of education, presence of comorbidities, type of surgical intervention (whether or not combined with radiotherapy), axillary surgery, chemotherapy, anti-hormonal therapy (whether or not completed), and breast reconstruction surgery (supplementary Table 1).

* Chi-squared tested (level of significance: 0.05 ).

** Patient-reported.

$\mathrm{X}$ interaction terms for chemotherapy (with or without targeted therapy) and anti-hormonal therapy were tested as well, but the models including these terms were not significantly better than the models without the interaction terms included. 



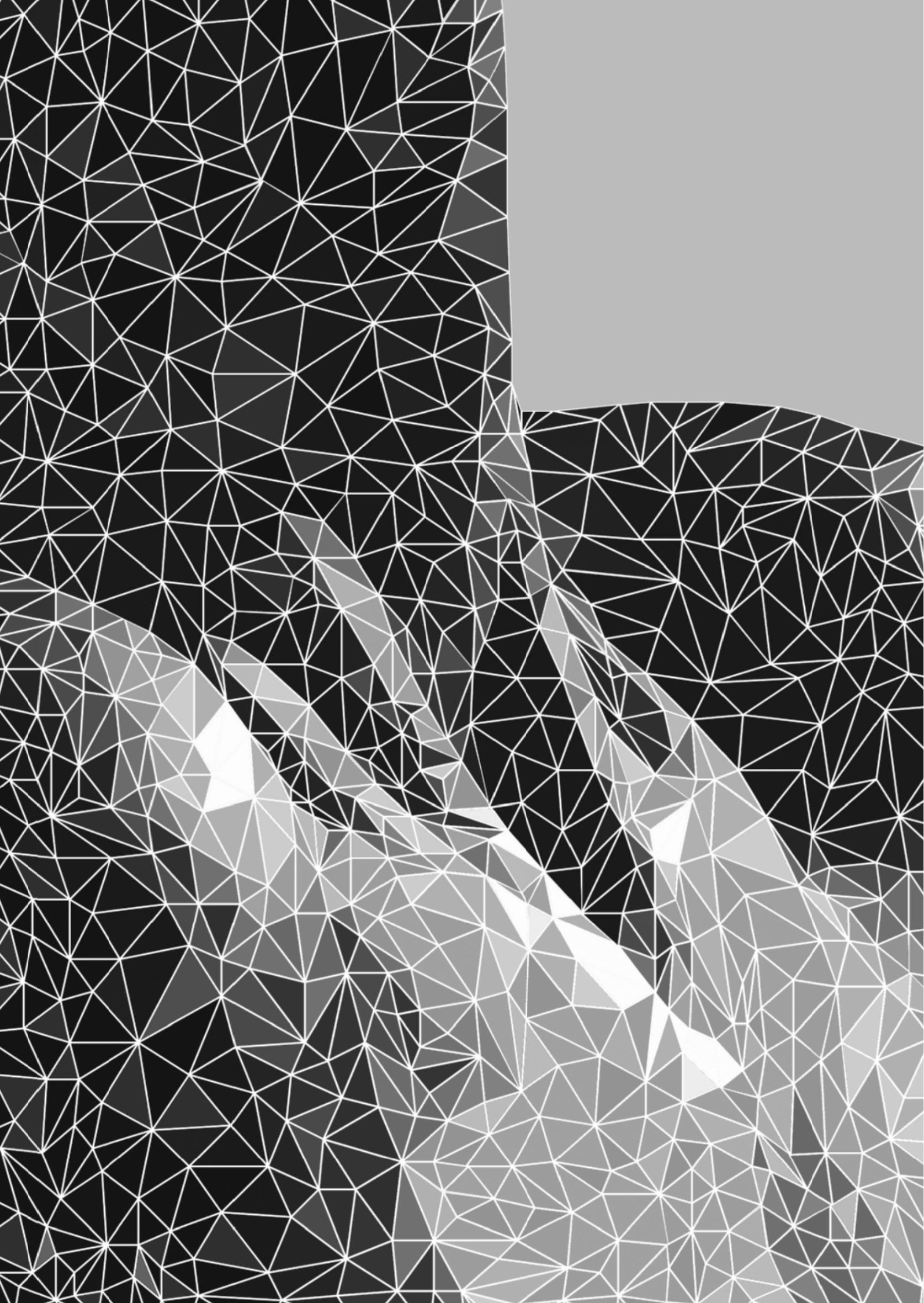




\section{CHAPTER 6}

THE IMPACT OF HEALTH SYMPTOMS ON HEALTH-RELATED QUALITY OF LIFE IN EARLY-STAGE BREAST CANCER SURVIVORS

K.M. de Ligt

M. Heins

J. Verloop

N.P.M. Ezendam

C.H. Smorenburg

J.C. Korevaar

S. Siesling

Submitted, May 2019 



\section{Abstract}

\section{Background:}

In breast cancer patients, treatment-related health symptoms can occur that may affect their Health-Related Quality of Life (HRQoL). This study aimed to determine the impact of health symptoms on HRQoL in breast cancer patients up to five years after diagnosis.

\section{Methods:}

Females surgically treated for early-stage breast cancer diagnosed between 2012-2016 ( $n=876$ ) were selected from the Netherlands Cancer Registry and asked to complete a survey about current health symptoms ('Symptoms and Perceptions questionnaire', SaP) and HRQoL ('EORTCQLQ-C30'). From the latter, five functioning scales and global health measure were included. Mean scores were compared to norm population scores (T-test). Multivariable linear regression analyses were performed to determine the association between health symptoms and global health and functioning.

\section{Results:}

404 patients $(46 \%)$ responded. The median age was $62.2 \pm 10.9$ years. Respondents had significant lower mean scores for role, cognitive, emotional, and social functioning than the general population. The most frequently reported health symptoms were musculoskeletal (including pain/complaints in lower/upper extremities, back, and neck; 71\%) and central nervous system symptoms (including concentration impairment, dizziness, neuralgia; $66 \%)$, and fatigue (63\%). While most symptoms affected functioning, irrespective of time since diagnosis, especially fatigue, musculoskeletal, central nervous system, and gastro-intestinal symptoms were significantly associated $(p<0.05)$ with lower functioning.

\section{Discussion:}

The majority of health symptoms that occur after breast cancer treatment were associated with lower functioning of patients in daily life. This urges healthcare providers to support breast cancer patients in alleviating or coping with health symptoms, even years after end of treatment, to improve their functioning. 


\section{Introduction}

For early-stage breast cancer, five-year survival rates are relatively high and have been increasing over the recent years $[1,2]$, with current rates in Europe and North America exceeding $85 \%$ $[1,3]$. This is mainly due to early detection by improved screening $[2,4]$ and improvements in multidisciplinary treatment $[4,5]$. Although breast cancer survivors report a relatively high healthrelated quality of life (HRQOL) [6-9], effects of breast cancer and its treatment may lower this HRQoL.These effects can impact all aspects of life, including physical, emotional, psychosocial, and cognitive well-being [10-12], and include lymphedema, pain and movement restrictions in the arm and shoulder [10,12-14], premature menopause, neuropathy [11-15], bone loss [10, 13, $14]$, cardiotoxic effects $[10,13,14]$, fatigue, insomnia, depression, cognitive dysfunction [10-12, $14,15]$, and sexual problems [10, 11, 14]. In general, a higher symptom burden was associated with lower HRQoL $[16,17]$.

Clinical guidelines recommend that patients receive follow-up care up to a minimum of five years after diagnosis to detect recurrent disease and to manage the physical and psychosocial effects of breast cancer and its treatment[18-20]. Even though survivorship care has become an increasingly important part of breast cancer care, there are concerns that benefits in treatment of breast cancer do not lead to similar benefits in psychosocial, functional, and sexual well-being [21]. That is, both the detection and management of late and side effects of breast cancer and its treatment is widely addressed as a research priority for the recent future [22, 23]. Patient Reported Outcome Measures (PROMs) are suggested as symptom detection method [21, 23], but current implementation in daily practice is hampered $[21,24,25]$. Furthermore, support in coping with symptoms may be insufficient, as patients reported health symptoms consistently over time years after diagnosis [9, $11,26,27]$. Two studies in a review by $\mathrm{Wu}$ et al. describe that $92 \%$ of patients reported residual symptoms one year after diagnosis, and $61 \%$ reporting pain, fatigue, and sleep disturbance up to 5 years after diagnosis [27]. Large unmet needs for information, detection, and management of physical impairments [9, 12], cognitive impairments [9], sexual functioning and enjoyment problems [9, 28], menopausal disorders [9], and anti-hormonal treatment effects as hot flashes [9, $29,30]$ were found. These unmet needs often mediate a lower HRQoL, and were associated with worse perceived physical and mental health [27, 31]. In general, residual treatment-related health symptoms were associated with lower QoL [27, 31], disability, and increased healthcare use [27].

To improve follow-up care, knowledge about all potential short and long-term treatment-related health symptoms and their impact on HRQoL is needed [23]. Specifically, it may be most effective to detect and, if possible, successfully unburden the health symptoms that are significantly associated with a lower HRQoL. The effects of health symptoms on HRQoL up to one year after treatment are commonly known [11,17], and for the long term, these effects were explored for frequently prevalent health symptoms as fatigue, sleep, depression, and pain $[9,27]$. Still, however, for the complete range of health symptoms that could occur in breast cancer patients $[10,13-15,17,26]$, the association of health symptoms with long-term $\mathrm{HRQoL}$ was not explored [17, 27, 32]. That while this is potentially important input for follow-up tools and guidance, which was addressed as a research need for breast cancer by the ESMO expert panel [23]. 
HRQoL is a multi-domain construct, typically including (overall) health perceptions, functioning, and symptoms. In this study we aimed to determine the impact of prevalent health symptoms on health perception and functioning in breast cancer patients up to five years after diagnosis.

\section{Methods}

\section{Study population}

This cross-sectional survey study utilised the data collected in our previous study [26]. Patients were selected from the Netherlands Cancer Registry (NCR), a national database that has documented population-based data about cancer incidence, diagnosis, and treatment [33]. We included surgically-treated female breast cancer patients (18 years or older), diagnosed with early-stage disease (stage I-III) between 2012 and 2016. For each participating hospital ( $\mathrm{N}=20)$, fifty patients were randomly selected $(\mathrm{N}=1000)$. In deliberation with these hospitals, patients who did not receive active follow-up, who were currently receiving treatment for secondary or recurrent disease, who could not read or write Dutch, or had no recent contact information, were excluded ( $n=124)$. Patients ( $n=876$ ) were then invited by the hospital administrations to complete the survey through the online PROFILES ('Patient-Reported Outcomes Following Initial treatment and Long term Evaluation of Survivorship') Registry survey application[34]. Invitations were sent between September 2017 and March 2018, responses were collected until May 2018. Participants gave consent for processing their coded responses and merging these with their clinical data available in the NCR. The use of NCR data in this study was approved by the NCR Privacy Review Board. Formal approval from an ethics committee was not required as the Dutch Medical Research (Human Subjects) Act did not apply for this study.

\section{Survey}

The survey (Appendix 1) consisted of three existing questionnaires and several self-composed questions: 1) HRQoL over the past four weeks; 2) health symptoms and diseases over the past year; 3 ) sociodemographic characteristic (age, highest completed level of education) and disease status and history (current treatment status, presence of comorbid disease at time of survey). First, HRQoL was measured through the EORTC-QLQ-C30 Quality of Life Questionnaire for Cancer. The QLQ-C30 includes a 2-item global health status/QoL scale, five multi-item functional scales (physical, role, emotional, cognitive, social functioning), and nine symptom scales or items. Answer scales ranged from 'not at all' to 'very much' in four steps. After transformation, scale scores range from 0 to 100, with high scores depicting good global health and functioning [35]. Second, 42 health symptoms were presented ('health problem present: yes/no') in the validated Symptoms and Perceptions questionnaire [36], supplemented with breast cancer-specific health symptoms from the literature [10,12-14]. Health symptoms were categorized in ten categories based on organ system. Third, comorbidities at time of survey were based on Sangha et al. [37], comprising examples of diseases (as diagnoses by a physician) rather than separate health symptoms. The following categories of diseases were included: other types of cancer, pulmonary, cardiovascular, gastrointestinal, urogenital, musculoskeletal, neurological, metabolic/coagulation, or infectious diseases. 
A Dutch normative population sample ( $n=1.105$ women, surveyed in 2013) was retrieved from CentERdata [38]. Breast cancer patients were not excluded from this population cohort, although we did look into the results for the population cohort without cancer patients as part of a sensitivity analysis.

\section{Analyses}

The analyses consisted of the following steps. First, the respondent characteristics (age, year of diagnosis, type of surgery, stage of disease, and type of hospital) were compared to non-respondent characteristics to assess generalizability (chi-square, level of significance $p<0.05$ ). Subsequently, respondent characteristics, health symptoms, and global health and functioning were reported. Second, multivariable linear regression analyses were performed to determine the effect of health symptoms on global health and functioning. Variables were selected based on significance; levels of significance of 0.10 and 0.05 were applicable for univariable and multivariable analyses, respectively. Backward selection was applied to reach parsimonious models. We corrected for time since diagnosis $[6,39]$, age $[6,27,39]$, presence of comorbid disease(s) $[6,27]$, and level of education $[6,27]$; these variables were selected based on the literature and availability in our dataset. We also corrected for breast reconstruction, as we expected this treatment modality had an independent and positive effect on HRQoL $[40,41]$. We did not correct for other treatment modalities.

Towards further interpretation, mean HRQoL scores for the respondents and norm population were compared through T-testing. Cohorts were matched 1:1 based on age (categories: $<50,50-$ $59,60-69,70+)$. Furthermore, mean HRQoL scores were stratified by time since diagnosis $(>2$, $2-4,4+$ years) and tested through one-way ANOVA. For both, a level of significance of $p<0.05$ was practised.

All analyses were performed in STATA SE14.2 [42].

\section{Results}

Completed surveys were received from $46 \%$ of invited patients (404/876). Respondents and nonrespondents did not differ significantly based on patient and treatment characteristics. However, compared to the invited population, respondents were slightly underrepresented in the youngest and oldest age category ( $<50$ years, $20 \%$ respondents vs $25 \%$ non-respondents; $70+$ years, $17 \%$ respondents vs $23 \%$ non-respondents; $p=0.010$, Supplementary Table 1 ).

Table 1 reports the respondents' patient, tumour, and treatment characteristics. Mean age was $62.0 \pm 10.9$ years, and one or more than one comorbidity was present in $48 \%$ of patients at time of survey. Patients had been treated with either breast conserving surgery (59\%) or mastectomy (41\%). Additionally to surgery, patients had received treatment with radiotherapy $(72 \%)$, chemotherapy (49\%), and anti-hormonal therapy (57\%). Table 2 presents patient-reported health symptoms categorized by organ system. The most commonly reported were health symptoms of the musculoskeletal system (71\%) and central nervous system (66\%), and fatigue (63\%). 
Table 1: Respondent characteristics ( $n=404)$

\begin{tabular}{|c|c|c|}
\hline & $N(404)$ & $\%$ \\
\hline \multicolumn{3}{|c|}{ Patient characteristics } \\
\hline \multicolumn{3}{|l|}{ Age (in years) at time of survey } \\
\hline $\begin{array}{l}\text { Mean } \\
\text { (SD,range) }\end{array}$ & $\begin{array}{c}62.20 \\
(11.0,27.5-91.6)\end{array}$ & \\
\hline$<50$ & 57 & 14 \\
\hline $50-59$ & 109 & 27 \\
\hline $60-69$ & 136 & 33 \\
\hline $70+$ & 102 & 25 \\
\hline \multicolumn{3}{|c|}{ Time (in years) between diagnosis and survey } \\
\hline$<2$ & 83 & 21 \\
\hline $2-4$ & 177 & 44 \\
\hline$>4$ & 144 & 36 \\
\hline \multicolumn{3}{|c|}{ Highest completed level of educationx $* *$} \\
\hline Secondary education or lower & 122 & 30 \\
\hline Medium vocational training & 170 & 42 \\
\hline High vocational training & 108 & 27 \\
\hline \multicolumn{3}{|l|}{ Number of comorbidities $\mathrm{x} * *$} \\
\hline 0 & 188 & 47 \\
\hline 1 & 131 & 33 \\
\hline $2 \geq$ & 61 & 15 \\
\hline unknown & 24 & 6 \\
\hline \multicolumn{3}{|c|}{ Tumour characteristics } \\
\hline \multicolumn{3}{|l|}{ Year of diagnosis } \\
\hline 2012 & 54 & 13 \\
\hline 2013 & 92 & 23 \\
\hline 2014 & 86 & 21 \\
\hline 2015 & 89 & 22 \\
\hline 2016 & 83 & 21 \\
\hline \multicolumn{3}{|l|}{ Stage } \\
\hline 1 & 186 & 46 \\
\hline II & 174 & 43 \\
\hline III & 44 & 11 \\
\hline \multicolumn{3}{|l|}{ Hormone-receptor status $\mathrm{x}$} \\
\hline HR-positive & 287 & 71 \\
\hline HR-mixed & 53 & 13 \\
\hline HR-negative & 62 & 15 \\
\hline \multicolumn{3}{|l|}{ Tumour grade $x$} \\
\hline 1 & 95 & 24 \\
\hline 2 & 176 & 44 \\
\hline 3 & 95 & 24 \\
\hline
\end{tabular}

Table 1 continues on next page 
Continuation of table 1

\begin{tabular}{|c|c|c|}
\hline & $N(404)$ & $\%$ \\
\hline \multicolumn{3}{|c|}{ Treatment characteristics } \\
\hline \multicolumn{3}{|l|}{ Treatment status at time of survey } \\
\hline Completed & 180 & 45 \\
\hline Currently receiving anti-hormonal therapy & 173 & 43 \\
\hline Currently receiving other treatment & 29 & 7 \\
\hline \multicolumn{3}{|l|}{ Surgery } \\
\hline Breast conserving surgery & 238 & 59 \\
\hline Mastectomy & 160 & 40 \\
\hline Axillary dissection & 85 & 21 \\
\hline Immediate breast reconstruction & 36 & 9 \\
\hline \multicolumn{3}{|l|}{ Adjuvant treatment: } \\
\hline Radiotherapy & 291 & 72 \\
\hline Chemotherapy & 196 & 49 \\
\hline With trastuzumab & 50 & 12 \\
\hline Anti-hormonal therapy & 232 & 57 \\
\hline \multicolumn{3}{|c|}{ Hospital characteristics } \\
\hline \multicolumn{3}{|l|}{ Hospital type ${ }^{a}$} \\
\hline General hospital & 166 & 41 \\
\hline Teaching/academic hospital & 238 & 59 \\
\hline \multicolumn{3}{|l|}{ Hospital volume $^{b}$} \\
\hline Low & 157 & 39 \\
\hline Medium & 88 & 22 \\
\hline High & 159 & 39 \\
\hline
\end{tabular}

$\mathrm{x}$ Totals do not add up due to missing values.

** Patient-reported.

a Hospitals were categorized as general, teaching, or academic hospitals.

b Number of surgical treated non-metastatic breast cancer patients per year (average over 2012-2016), categorized as low $(<100)$, medium (100-149), and high (>150) volume.

Table 2: categories of patient-reported symptoms

\begin{tabular}{|c|c|c|}
\hline & $N(404)$ & $\%$ \\
\hline Fatigue & 256 & 63 \\
\hline $\begin{array}{l}\text { Cardiac: } \\
\quad \text { Palpitations, chest pain or tightness }\end{array}$ & 77 & 19 \\
\hline $\begin{array}{l}\text { Respiratory: } \\
\text { Cough, complaints in the nose, shortness of breath. }\end{array}$ & 124 & 31 \\
\hline $\begin{array}{l}\text { Gastrointestinal: } \\
\quad \text { Dry mouth, diarrhea/constipation, gastric or abdominal complaints, nausea. }\end{array}$ & 160 & 40 \\
\hline Urinary complaints: difficulties with urinating in general & 34 & 8 \\
\hline $\begin{array}{l}\text { Central nervous system: } \\
\text { Memory/concentration, tingling hands/feet (neuralgia), irritation of eyes, } \\
\text { dizziness/vertigo, headache, earache or ear complaints, hypersensitivity to } \\
\text { light or sound. }\end{array}$ & 267 & 66 \\
\hline
\end{tabular}

Table 2 continues on next page 


\begin{tabular}{lcc}
\hline $\begin{array}{l}\text { Skin: } \\
\text { Hair loss, skin problems. }\end{array}$ & 153 & 38 \\
\hline $\begin{array}{l}\text { Psychological: } \\
\text { Insomnia, agitation/irritability, anxiety, depressive feelings, sudden feelings of } \\
\text { stress or crisis, increased in use of drugs or alcohol. }\end{array}$ & 214 \\
\hline $\begin{array}{l}\text { Reproductive system: } \\
\text { Menopausal complaints, weight increase/decrease, problems with sex or } \\
\text { sexuality, infertility. }\end{array}$ & 220 \\
\hline $\begin{array}{l}\text { Breast: } \\
\text { Hypersensitivity in breast area, pain/swelling scars in breast area, axillary } \\
\text { complaints (incl. lymphoedema), skin problems in breast area. }\end{array}$ & 218 \\
\hline $\begin{array}{l}\text { Musculoskeletal: } \\
\text { Pain/complaints in upper extremities, pain/complaints in lower extremities, } \\
\text { neck or shoulder pain/complaints, myalgia/muscle strain, back pain/ } \\
\text { complaints, movement restrictions in arm, fractures. }\end{array}$ & 285 \\
\hline
\end{tabular}

Reported in De Ligt et al ${ }^{26}$

\section{HRQoL compared to norm population}

Figure 1 reports mean global health and functioning for both the respondents and general reference population (including cancer patients). The mean global health score was $76.3 \pm 17.2$ for respondents, which did not differ significantly from the general population (75.6 \pm 16.9$)$. Although mean scores for all individual functioning domains were 80 or higher, these scores were significantly lower than in the general population for role $(80.3 \pm 23.6$ vs $84.3 \pm 23.6 ; p=0.016)$, emotional ( $82.9 \pm 19.9$ vs $85.4 \pm 16.9 ; p=0.053)$, cognitive ( $80.6 \pm: 21.9$ vs $90.0 \pm 15.9 ; p<0.001)$, and social ( $85.6 \pm 21.6$ vs $91.8 \pm 17.7 ; p<0.001)$ functioning. Excluding cancer patients from the general population sample did not alter our finding.

When zooming in at the younger population (age $<50$ years), physical functioning was significantly better in younger patients ( 5 points difference), while cognitive (11 points) and social (9 points) were significantly worse than in older patients (results not shown). When stratified by years since diagnosis, the mean HRQoL scores did not statistically differ over time (Supplementary Table 2).

Last, when stratified by number of symptom categories, mean scores were significantly lower when more symptoms were reported for all domains (Supplementary Table 3). Only 12 respondents (3\%) reported zero symptoms in either of the eleven categories of symptoms, while $37 \%, 50 \%$, and $11 \%$ reported $1-4,5-8$, and $8-11$ categories of symptoms, respectively. Mean global health scores ranged between 91.7 and 64.9 when zero and 8-11 categories of health symptoms were reported $(p<0.001)$, respectively. For the functioning scales, mean scores ranged between 100 (social functioning) and 63.1 (cognitive functioning) for zero and 8-11 categories of health symptoms reported, respectively. Frequently reported dyads of health symptoms were reported in Supplementary Table 4. Especially fatigue, symptoms of the central nervous system, and musculoskeletal symptoms were reported frequently in combination with other symptoms. 


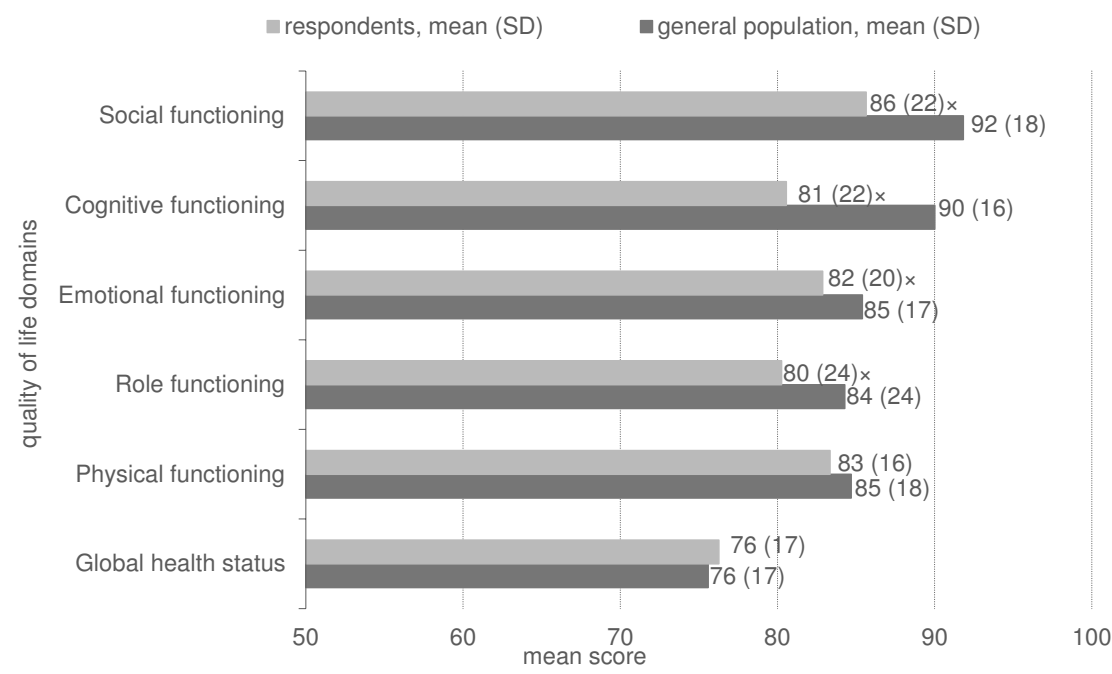

Figure 1: Mean HRQoL, compared to the general population

Scales range from 0 to 100 , with high scores depicting good global health and functioning

$x$ significant difference $(p<0.05$, T-test) respondents vs general population

\section{The effect of health symptoms on HRQoL scores}

Table 3 presents the associations between prevalent health symptoms and functioning, adjusted for covariates and after backward selection. $A \beta$ of $-x$ indicated a negative association between the health problem and global health or functioning of $x$ points. All included domains were significantly negatively associated with either one of the categories of health symptoms. Cognitive functioning was affected by a range of health symptoms, including cardiac $(\beta:-4.4, p=0.002)$, gastrointestinal $(\beta:-4.3, p=0.042)$, renal and urinary $(\beta:-9.7, p=0.006)$, central nervous system $(\beta:-16.2, p<0.001)$, and psychological $(\beta:-5.2, p=0.017)$ health symptoms. Furthermore, health symptoms burdened different aspects of quality of life. For instance, musculoskeletal health symptoms affected global health status ( $\beta:-4.2, p=<0.028)$, and physical $(\beta:-7.6, p=<0.001)$, role $(\beta:-9.5, p<0.001)$, emotional $(\beta:-5.0, p=0.019)$, and social $(\beta:-6.3, p=0.016)$ functioning. Last, associations were of different magnitudes. The largest effects were found for the associations between health symptoms of the central nervous system and cognitive functioning $(\beta:-16.2, p<0.001)$, and fatigue and role functioning ( $\beta$ :-14.1, $p=<0.001)$.

Supplementary Table 5 includes all covariates included in the multivariate analyses. Time since diagnosis was not significantly associated with functioning. Higher age was associated with significantly lower physical functioning (60-69: $\beta:-6.8, p=0.002 ; 70+: \beta:-13.5, p<0.001$ ) and better cognitive functioning ( $60-69: \beta: 7.4, p=0.014 ; 70+: \beta:-7.8, p=0.018$ ). Presence of comorbid disease at time of survey was negatively associated with global health $(\beta:-5.5, p=0.001)$, physical functioning $(\beta:-6.1, p<0.001)$, and role functioning $(\beta:-8.5, p<0.001)$. Immediate breast reconstruction was associated with a higher global health $(\beta: 7.7, p=0.005)$ and emotional functioning $(\beta: 8.4, p=0.007)$. 
Table 3: association between health symptoms and HRQoL functioning domains through multivariable linear regression

\begin{tabular}{|c|c|c|c|c|c|c|c|}
\hline & \multirow{2}{*}{$\begin{array}{l}\text { Quality of life } \\
\qquad(\beta, C l) \rightarrow\end{array}$} & \multirow{2}{*}{$\begin{array}{c}\text { Global health } \\
\text { status }\end{array}$} & \multicolumn{5}{|c|}{ Functioning } \\
\hline & & & Physical & Role & Emotional & Cognitive & Social \\
\hline \multirow{11}{*}{ 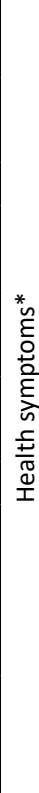 } & Fatigue & $\begin{array}{c}-9.4 \\
(-13.0 ;-5.8)\end{array}$ & $\begin{array}{c}-8.2 \\
(-11.2 ;-3.3)\end{array}$ & $\begin{array}{c}-14.1 \\
(-18.9 ;-9.3)\end{array}$ & & & $\begin{array}{c}-6.2 \\
(-11.4 ;-1.4)\end{array}$ \\
\hline & Cardiac & $\begin{array}{c}-4.4 \\
(-8.3 ;-0.5)\end{array}$ & & & $\begin{array}{c}-5.5 \\
(-9.9 ;-1.0)\end{array}$ & $\begin{array}{c}-5.7 \\
(-10.5 ;-0.8)\end{array}$ & \\
\hline & Respiratory & & & & & & \\
\hline & Gastrointestinal & $\begin{array}{c}-3.4 \\
(-6.8 ;-0.1)\end{array}$ & $\begin{array}{c}-5.3 \\
(-8.2--2.4)\end{array}$ & $\begin{array}{c}-7.8 \\
(-12.3 ;-3.3)\end{array}$ & & $\begin{array}{c}-4.3 \\
(-8.5 ;-0.1)\end{array}$ & \\
\hline & $\begin{array}{c}\text { Renal and } \\
\text { urinary }\end{array}$ & & & & & $\begin{array}{c}-9.7 \\
(-16.5 ;-2.8)\end{array}$ & \\
\hline & $\begin{array}{c}\text { Central nervous } \\
\text { system }\end{array}$ & $\begin{array}{c}-5.5 \\
(-9.3 ;-1.7)\end{array}$ & & & $\begin{array}{c}-8.3 \\
(-12.5 ;-4.1)\end{array}$ & $\begin{array}{c}-16.2 \\
(-20.8 ;-11.6)\end{array}$ & $\begin{array}{c}-6.1 \\
(-11.2 ;-0.9)\end{array}$ \\
\hline & Skin & & & & & & \\
\hline & Psychological & & & & $\begin{array}{c}-12.7 \\
(-12.7 ;-8.8)\end{array}$ & $\begin{array}{c}-5.2 \\
(-9.3 ;-0.9)\end{array}$ & $\begin{array}{c}-6.2 \\
(-10.8 ;-1.7)\end{array}$ \\
\hline & $\begin{array}{l}\text { Reproductive } \\
\text { system }\end{array}$ & & & & & & \\
\hline & Breast & & & & & & \\
\hline & Musculoskeletal & $\begin{array}{c}-4.2 \\
(-8.0 ;-0.5)\end{array}$ & $\begin{array}{c}-7.6 \\
(-11.0 ;-4.3)\end{array}$ & $\begin{array}{c}-9.5 \\
(-14.7 ;-4.3)\end{array}$ & $\begin{array}{c}-5.0 \\
(-9.1 ;-0.8)\end{array}$ & & $\begin{array}{c}-6.3 \\
(-11.4 ;-1.2)\end{array}$ \\
\hline
\end{tabular}

$\mathrm{Cl}$ : confidence interval

$\beta$ : association of $x$ points of health problem on HRQoL.

Cells are empty when factors were not significant in univariate testing and thus not included in multivariate testing, or were excluded through backward selection in the multivariate analyses. Analyses were corrected for age at time of survey, presence of comorbid diseases, highest completed level of education at time of diagnosis, and breast reconstruction.

* In categories. Reference categories for health symptoms were patients who not reported health symptoms in this category.

\section{Discussion}

In our study, the mean global health in breast cancer patients up to five years after diagnosis was comparable to that in the general population (76.3 vs 75.6). Mean scores of 80 or higher were found for functioning, although these were significantly lower than in the general population for role, emotional, cognitive, and social functioning. Almost all reported health symptoms were significantly negatively associated with either one of the functioning scales, but were most pronounced for musculoskeletal health symptoms, fatigue, health symptoms in the central nervous system, and gastro-intestinal health symptoms.

We found that HRQoL in breast cancer survivors up to five years after diagnosis is relatively high compared to that in the general population, alike previous literature [6, 7]. In line with previous 
EORTC-QLQ-C30 measurements, standard deviations were quite wide [17, 32, 43-45]. We believe that especially the domains for which a lower mean score than in the general population was reported are important to breast cancer patients, although not all reported differences may be regarded clinically relevant $[46,47]$. For cognitive functioning, we found a difference of 9 points; a mean difference of 9-14 points was regarded a medium effect, suggesting the difference we found is clinically relevant. For social functioning, we found a difference of 6 points, which is regarded a small effect (5-11 points mean difference). For the other domains, differences found were of trivial effect and thus of less clinical relevance. However, as an individual may encounter effects simultaneously on multiple domains, we suggest trivial or small differences should not be immediately neglected. More specifically, our results describe as well that global health and functioning is significantly lower in respondents reporting a higher symptom prevalence. Especially fatigue, symptoms of the central nervous system, and musculoskeletal symptoms were reported frequently in combination with other symptoms. Thus, several small mean differences may add up to large and multifaceted effects on global health and functioning. We believe this deserves more awareness in clinical practise.

In line with the literature [44], we found no statistically significant difference between the respondents and the general population for global health and physical functioning. This may seem contradictory to the lowered functioning scores we found, but similar results were reported in other studies [27]. A possible explanation may be a re-evaluation of general health perception in breast cancer patients, or so-called 'response shift' [48]. However, it may be as well that the global health domain is a less discriminating measurement domain than other domains. Blome et al. [48] describe that it is assumed that HRQoL is a more or less universal concept, while in fact, a certain objective impairment does not necessarily lead to the same reduction of HRQoL in any patient.

Negative associations with functioning were especially found for musculoskeletal health symptoms, fatigue, health symptoms in the central nervous system, and gastro-intestinal health symptoms; these first two were found by Arndt et al. [32] as well. However, this study included patients up to one year after diagnosis, while after a year other symptoms than acute symptoms became relevant $[11,32]$. Furthermore, we found mean scores did not differ according to time since diagnosis. Literature found reduced HRQoL up to five years after diagnosis [43, 44], but improvements more onwards as well [39]. This underlines the importance of longitudinal data to determine time effects for HRQoL.

\section{Study limitations}

This study provides insight in global health and functioning in a multi-centre, population-based cohort of 404 breast cancer patients up to five years after diagnosis. However, some annotations need to be made. First, several forms of bias may apply to patient-reported data, including recall bias, report bias, selection bias, and survivorship bias. As a result of recall bias, the reported health symptoms may have been underrepresented when patients were not able to recall all health symptoms they encountered in the previous year. Although recall bias was not applicable for the assessment of HRQoL at time of survey, response shift may lead to different interpretations of $\mathrm{HRQ}$ oL over time [48]. As a result from our response rate (46\%), health symptoms may have been 
either overestimated if only patients with many health symptoms participated, or underestimated by reluctance towards reporting for instance sexuality problems [38]. Furthermore, we excluded patients who could not read or write Dutch, and thus risk not including a vulnerable part of the patient population: non-responding patients in observational patient-reported outcome studies have different socio-demographic and clinical characteristics, and may have systemically lower HRQoL scores [49]. As patients with less favourable socio-demographic characteristics may have been exposed to other risk factors affecting both breast cancer risk and HRQoL, information about healthy behaviour would be beneficial in further interpreting the results. As a result of survivorship bias, patients with relatively favourable disease characteristics may have been included.

\section{Implications for practice}

We were able to confirm that a higher symptom burden was associated with a lower HRQoL $[16,17]$. Therefore, it may be most effective to detect and manage or unburden the treatmentrelated health symptoms that were significantly associated with a lower HRQoL, and thus provide follow-up care that is valuable to the daily functioning of breast cancer patients. For instance, guidelines provides recommendations for physical therapy for the effects of musculoskeletal health symptoms as arm/shoulder function and lymphoedema $[18,19]$. However, such clear recommendations are not available for all reported negative associations. Fatigue was prevalent in $9-100 \%$ of cancer survivors $[9,15,17,26,27,44]$ and has a large impact on HRQoL and functioning $[9,15,17,27,32]$. Exercise, cognitive-behavioral therapy, and education about coping were found to be effective against cancer-related fatigue $[10,50,51]$, however, fatigue was found to be a very persistent problem, lasting up to ten years in one-fourth to one-third of breast cancer patients [15]. Moreover, of the $63 \%$ of respondents who reported fatigue, only a third reported this to her physician [26], suggesting under detection in many patients. Furthermore, a large negative association was found between health symptoms of the central nervous system and cognitive functioning, probably caused by the large proportion of patients (42.6\%) reporting memory and concentration symptoms. Even though cognitive dysfunction is frequently reported among (breast) cancer patients, not much is known about its etiology, and proven effective interventions are lacking $[10,15,52]$. Last, we found negative associations between gastro-intestinal problems and global health, and physical, role, and cognitive functioning. In literature, gastrointestinal symptoms as diarrhoea, nausea, appetite loss, and constipation were reported less frequently over time, by $15 \%$ to $20 \%$ of patients [44], which we could confirm [26]. Gastric or abdominal complaints were reported in $13 \%$ of patients. However, over $50 \%$ of them reported health-care use for this symptom, which was nearly the highest reported use of care for the range of included symptoms [26]. Literature reported that the majority of gastro-complaints declined over time [11, $44]$, still, the high use of care could indicate many patients struggle with these symptoms long after diagnosis.

These examples illustrate the importance of detection and acknowledgement of health symptoms, but also the importance of research towards successful detection and intervention for these health symptoms [53], so that guidelines can provide guidance in dealing with them. Cardoso et al. defined survivorship research as a priority research area for breast cancer, including follow-up tools to assess quality of life in long-term survivors [23]. 
We measured symptoms through the SaP questionnaire [36], rather than through the symptom domains included in the EORTC QLQ-C30. The Sap assesses a broader range of non-specific symptoms, its aim is to include symptoms from all relevant organ systems. We hypothesized that a broad selection of symptoms is of relevance for longer-term survivors [27], broader than is currently included in the cancer-specific QLQ-C30. Our results, described in more detail in our previous paper [26], confirm this hypothesis. For instance, menopausal complaints (40\%) and memory and concentration complaints (43\%) were commonly prevalent in our respondents, but are not included in the QLQ-C30 and only to a limited extent in the EORTC BR-23 breast cancer module. This hypothesis was later confirmed by Van Leeuwen et al. [11]. Therefore, for further research, we endorse the suggestion made by Van Leeuwen et al: development of a survey specifically for cancer survivors would address a gap in assessing issues that are of a more chronic nature [11]. Also, according to literature, young patients might be at a higher risk of poorer HRQoL $[27,44]$. Although younger patients were underrepresented in our study, we found lower HRQoL in younger patients as well. Thus, both care and research should focus more on sub groups within survivorship that have a higher risk of deprived HRQoL.

\section{Conclusion}

Early-stage breast cancer patients up to five years after diagnosis had significant lower mean scores than the general population for all functioning domains but physical functioning, and scores did not differ statistically over the years since diagnosis. The majority of health symptoms prevalent after breast cancer treatment was associated with lower functioning of patients in daily life. This urges healthcare providers to support patients in alleviating or coping with treatment-related health symptoms, even years after end of treatment, to improve functioning of breast cancer survivors. 


\section{References}

1. Howlader N, Noone AM, Krapcho M, Miller D, Bishop K, Kosary CL, et al. SEER Cancer Statistics Review, 1975-2014 Bethesda, MD: National Cancer Institute; 2017 [updated Based on November 2016 SEER data submission, posted to the SEER web site April 2017. Available from: https://seer.cancer.gov/csr/1975_2014/.

2. Coleman MP, Quaresma M, Berrino F, Lutz JM, De Angelis R, Capocaccia R, et al. Cancer survival in five continents: a worldwide population-based study (CONCORD). Lancet Oncology. 2008;9:730-56.

3. Janssen-Heijnen MLG, van Steenbergen LN, Voogd AC, Tjan-Heijnen VCG, Nijhuis PH, Poortmans PM, et al. Small but significant excess mortality compared with the general population for long-term survivors of breast cancer in the Netherlands. Annals of Oncology. 2014;25:64-8.

4. Soerjomataram I, Louwman MWJ, Ribot JG, Roukema JA, Coebergh JWW. An overview of prognostic factors for long-term survivors of breast cancer. Breast Cancer Res Treat. 2008;107:309-30.

5. Murawa P, Murawa D, Adamczyk A, Połom K. Breast cancer: Actual methods of treatment and future trends. Reports of Practical Oncology and Radiotherapy. 2014;19:165-72.

6. Mols F, Vingerhoets AJJM, Coebergh JW, van de Poll-Franse LV. Quality of life among long-term breast cancer survivors: A systematic review. European Journal of Cancer. 2005;41:2613-9.

7. Ganz PA, Desmond KA, Leedham B, Rowland JH, Meyerowitz BE, Belin TR. Quality of Life in Long-Term, Disease-Free Survivors of Breast Cancer: a Follow-up Study. J Natl Cancer Inst. 2002;94:39-49.

8. Tan ML, Idris BD, Teo LW, Loh SY, Seow GC, Chia YY, et al. Validation of EORTC QLQ-C30 and QLQ-BR23 questionnaires in the measurement of quality of life of breast cancer patients in Singapore. Asia-Pacific Journal of Oncology Nursing. 2014;1(1).

9. Schmidt ME, Wiskemann J, Steindorf K. Quality of life, problems, and needs of disease-free breast cancer survivors 5 years after diagnosis. Quality of Life Research. 2018;27:2077-86.

10. Kenyon M, Mayer DK, Owens AK. Late and Long-Term Effects of Breast Cancer Treatment and Surveillance Management for the General Practitioner. Journal of Obstetric, Gynecologic \& Neonatal Nursing. 2014;43:382-98.

11. van Leeuwen $M$, Husson O, Alberti P, Arraras JI, Chinot OL, Costantini A, et al. Understanding the quality of life (QOL) issues in survivors of cancer: towards the development of an EORTC QOL cancer survivorship questionnaire. Health and Quality of Life Outcomes. 2018;16(1).

12. Binkley JM, Harris SR, Levangie PK, Pearl M, Guglielmino J, Kraus V, et al. Patient Perspectives on Breast Cancer Treatment Side Effects and the Prospective Surveillance Model for Physical Rehabilitation for Women With Breast Cancer. Cancer. 2012;118:2207-16.

13. Agrawal S. Late effects of cancer treatment in breast cancer survivors. South Asian Journal of cancer. 2014;3(2).

14. Ewertz $M$, Jensen $A B$. Late effects of breast cancer treatment and potentials for rehabilitation. Acta Oncologica. 2011;50:187-93.

15. Pinto AC, de Azambujab E. Improving quality of life after breast cancer: Dealing with symptoms. Maturitas. 2011;70:343-8.

16. Avis NE, Levine B, Marshall SA, Ip EH. Longitudinal Examination of Symptom Profiles Among Breast Cancer Survivors. Journal of Pain and Symptom Management. 2017;53(4):703-10.

17. Janz NK, Mujahid M, Chung LK, Lantz PM, Hawley ST, Morrow M, et al. Symptom Experience and Quality of Life of Women Following Breast Cancer Treatment. Journal of Women's Health. 2007;16(9).

18. Netherlands Comprehensive Cancer Organisation (IKNL). National guideline on Breast Cancer. Netherlands Comprehensive Cancer Organisation (IKNL),,; 2012.

19. Runowicz CD, Leach CR, Henry NL, Henry KS, Mackey HT, Cowens-Alvarado RL, et al. American Cancer Society/American Society of Clinical Oncology Breast Cancer Survivorship Care Guideline. Journal of Clinical Oncology. 2016;34(6):611-35.

20. Senkus E, Kyriakides S, Ohno S, Penault-Llorca F, Poortmans P, Rutgers E, et al. Primary breast cancer: ESMO Clinical Practice Guidelines for diagnosis, treatment and follow-up. Annals of Oncology. 2015;26:8-30. 
21. Fallowfield L, Jenkins V. Psychosocial/Survivorship Issues in Breast Cancer: Are We Doing Better? J Natl Cancer Inst. 2015;107(1):dju335.

22. National Cancer Research Institute \& James Lind Alliance. UK Top 10 living with and beyond cancer research priorities. 2018.

23. Cardoso F, Harbeck N, Barrios CH, Bergh J, Cortés J, El Saghir N, et al. Research needs in breast cancer. Annals of Oncology. 2017;28:208-17.

24. Black N. Patient reported outcome measures could help transform healthcare. British Medical Journal. 2013;346:f167.

25. Tevis SE, James TA, Kuerer HM, Pusic AL, Yao KA, Merlino J, et al. Patient-Reported Outcomes for Breast Cancer. Annals of Surgical Oncology. 2018;25:2839-45.

26. de Ligt KM, Heins M, Verloop J, Smorenburg CH, Korevaar JC, Siesling S. Patient-reported health problems and healthcare use after treatment for early-stage breast cancer. The Breast. 2019;4(46):4-11.

27. Wu HS, Harden JK. Symptom Burden and Quality of Life in Survivorship: A Review of the Literature. Cancer Nursing. 2014;38(1).

28. Stabile C, Goldfarb S, Baser RE, Goldfrank DJ, Abu-Rustum NR, Barakat RR, et al. Sexual Health Needs and Educational Intervention Preferences for Women with Cancer. Breast Cancer Res Treat. 2017;165(1):77-84.

29. Bluethmann SM, Murphy CC, Tiro JA, Mollica MA, Vernon SW, Bartholomew LK. Deconstructing Decisions to Initiate, Maintain, or Discontinue Adjuvant Endocrine Therapy in Breast Cancer Survivors: A Mixed-Methods Study. Oncol Nurs Forum. 2017;44(3):E101-e10.

30. Brauer ER, Ganz PA, Pieters HC. "Winging It": How Older Breast Cancer Survivors Persist With Aromatase Inhibitor Treatment. Journal of oncology practice. 2016;12(12):e991-e1000.

31. Mayer DK, Nasso SF, Earp JA. Defining cancer survivors, their needs, and perspectives on survivorship health care in the USA. The Lancet. 2017;18(1):e11-e8.

32. Arndt V, Stegmaier C, Ziegler H, Brenner H. A Population-Based Study of the Impact of Specific Symptoms on Quality of Life in Women With Breast Cancer 1 Year After Diagnosis. Cancer. 2006;107:2496-503.

33. Netherlands Comprehensive Cancer Organisation (IKNL). About the registration 2018 [Available from: https://www.cijfersoverkanker.nl/about-the-registration-37.html.

34. van de Poll-Franse LV, Horevoorts N, Eenbergen M, Denollet J, Roukema JA, Aaronson NK, et al. The Patient Reported Outcomes Following Initial treatment and Long term Evaluation of Survivorship registry: Scope, rationale and design of an infrastructure for the study of physical and psychosocial outcomes in cancer survivorship cohorts. European journal of cancer (Oxford, England : 1990). 2011;47(14):2188-94.

35. Aaronson NK, Ahmedzai S, Bergman B, Bullinger M, Cull A, Duez NJ, et al. The European Organisation for Research and Treatment of Cancer QLQ-C30: A quality-of-life instrument for use in international clinical trials in oncology. J Natl Cancer Inst. 1993;85:365-76.

36. Yzermans J, Baliatsas C, van Dulmen S, van Kamp I. Assessing non-specific symptoms in epidemiological studies: development and validation of the symptoms and perceptions (SaP) questionnaire. International journal of hygiene and environmental health. 2016;219(1):53-65.

37. Sangha O, Stucki G, Liang MH, Fossel AH, Katz JN. The Self-Administered Comorbidity Questionnaire:A New Method to Assess Comorbidity for Clinicaland Health Services Research. Arthritis \& Reumatism. 2003;49(2):156-63.

38. van de Poll-Franse LV, Mols F, Gundy CM, Creutzberg CL, Nout RA, Verdonck-de Leeuw IM, et al. Normative data for the EORTC QLQ-C30 and EORTC-sexuality items in the general Dutch population. European journal of cancer (Oxford, England : 1990). 2011.

39. Hamer J, McDonald R, LZ, Verma S, Leahey A, Ecclestone C, et al. Quality of life (QOL) and symptom burden (SB) in patients with breast cancer. Supportive care in cancer : official journal of the Multinational Association of Supportive Care in Cancer. 2017;25:409-19.

40. Santosa KB, Qi J, Kim HM, Hamill JB, Wilkins EG, Pusic AL. Long-term Patient-Reported Outcomes in Postmastectomy Breast Reconstruction. JAMA surgery. 2018;153(10):891-9.

41. Lagendijk M, van Egdom LSE, Richel C, van Leeuwen N, Verhoef C, Lingsma HF, et al. Patient reported outcome measures in breast cancer patients. European Journal of Surgical Oncology. 2018;44:963-8.

42. StataCorp. Stata Statistical Software: Release 14. In: College Station TSL, ,, editor. 2015. 
43. Holzner B, Kemmler G, Kopp G, Moschen R, Schweigkofler H, Dünser M, et al. Quality of Life in Breast Cancer Patients-Not Enough Attention for Long-Term Survivors? Psychosomatics. 2001;42(117-123).

44. Arndt V, Merx H, Stegmaier C, Ziegler H, Brenner H. Persistence of Restrictions in Quality of Life From the First to the Third Year After Diagnosis in Women With Breast Cancer. Journal of Clinical Oncology. 2005;23(22):4945-53.

45. Härtl K, Janni W, Kästner R, Sommer H, Strobl B, Rack B, et al. Impact of medical and demographic factors on long-term quality of life and body image of breast cancer patients. Annals of Oncology. 2003;14:1064-71.

46. Osoba D, Rodrigues G, Myles J, Zee B, Pater J. Interpreting the Significance of Changes in Health-Related Quality-of-Life Scores. J Clin Oncol. 1998;16:139-44.

47. Cocks K, King MT, Velikova G, St-James MM, Fayers PM, Brown JM. Evidence-Based Guidelines for Determination of Sample Size and Interpretation of the European Organisation for the Research and Treatment of Cancer Quality of Life Questionnaire Core 30. Journal of Clinical Oncology. 2011;29(1):89-96.

48. Blome C, Augustin M. Measuring Change in Quality of Life: Bias in Prospective and Retrospective Evaluation. Value in Health. 2015;18:110-5.

49. de Rooij BH, Ezendam NPM, Mols F, Vissers PAJ, Thong MSY, Vlooswijk CCP, et al. Cancer survivors not participating in observational patient-reported outcome studies have a lower survival compared to participants: the population-based PROFILES registry. Qual Life Res. 2018;27(12):3313-24.

50. Barsevick AM, Newhall T, Brown S. Management of Cancer-Related Fatigue. Clin J Oncol Nurs. 2008;12:21-5.

51. Hilfiker R, Meichtry A, Eicher M, Nilsson Balfe L, Knols RH, Verra ML, et al. Exercise and other nonpharmaceutical interventions for cancer-related fatigue in patients during or after cancer treatment: a systematic review incorporating an indirect-comparisons meta-analysis. Br J Sports Med. 2018;52:651-8.

52. Phillips KM, Jim HS, Small BJ, Laronga C, Andrykowski MA, Jacobsen PB. Cognitive Functioning After Cancer Treatment: A Three-Year Longitudinal Comparison of Breast Cancer Survivors Treated with Chemotherapy or Radiation and Non-cancer Controls. 118. 2012;7:1925-32.

53. Breast Cancer Research Foundation. Improving Outcomes for Breast Cancer Survivors: Perspectives on Research Challenges and Opportunities. PA G, editor2015. 


\section{Appendix 1: Survey}

0 What is your date of birth?

....-19..

1. In general, would you say your health is:

1 - Excellent

2 - Very good

3 - Good

4 - Fair

5 - Poor

2. I seem to get sick a little more easily than other people

Definitely true - Mostly true - Don't know - Mostly false - Definitely false

3. I am as healthy as anybody I know

Definitely true - Mostly true - Don't know - Mostly false - Definitely false

4. I expect my health to get worse

Definitely true - Mostly true - Don't know - Mostly false - Definitely false

5. My health is excellent

Definitely true - Mostly true - Don't know - Mostly false - Definitely false

6. Compared to one year ago, how would you rate your health in general now?

1 - Much better now than one year ago

2 - Somewhat better now than one year ago

3 - About the same

4 - Somewhat worse now than one year ago

5 - Much worse now than one year ago

7. Do you currently suffer from one or more of the diseases or conditions listed below?

Any other type of cancer - pulmonary disease - cardiovascular disease - gastrointestinal disease - disease of the urinary or reproductive system - musculoskeletal disease - central nervous system metabolic disorder or coagulopathy - infectious disease - none - other

In case you do: for which of these did you receive treatment?

8. Which of the following health problems have you experienced over the recent year? And for which of these health problems did you visit a primary care physician or other doctor?

\section{Palpitations}

Chest pain/tightness

Shortness of breath

Nasal complaints

Cough

Stomach/abdomen complaints

Dry mouth

Nausea

Diarrhoea/constipation

Urinary complaints

Memory/concentration

Irritation to the eye(s) 


\section{Dizziness}

Head ache

Earache or complaints

Tingling hands/feet

Hypersensitivity to light

Skin problems

Hair loss

Anxiety

Depressive feelings

Sudden feelings of stress or crisis

Agitation/irritability

Insomnia

Increased use of drugs or alcohol

Problems with sex or sexuality

Menopausal complaints

Weight increase/decrease

Infertility

Pain/swelling of scars

Hypersensitivity in the breast area

Complaints axilla (incl. lymphoedema)

Skin problems in the breast area

Neck or shoulder pain/complaints

Back pain/complaints

Pain/complaints of the upper extremities

Fractures

Pain/complaints of the lower extremities

Myalgia/muscle strain

Movement restrictions of the arm

9. What was your key health problem in the list above? Fill in only one health problem.

(...)

13. What is your highest completed level of education? (i.e. with a diploma or certificate)

No education - lower education - middle education - higher education - other

14. Do you have a partner?

Yes - No

15. Do you have children?

Yes, they live with me - Yes, they live on their own - No

16. What description is most applicable to you at this moment? (please tick one answer)

Attending school/education - paid employment - unemployed/seeking work - incapacitated homemaker - retirement

17. Are you currently under treatment for your breast cancer?

No, I have finished my treatment - Yes, I am currently receiving anti-hormonal treatment - Yes, I am receiving [fill in the treatment you are currently receiving] 
Supplementary Table 1: characteristics respondents $(n=408)$ vs non respondents $(n=592)$

\begin{tabular}{|c|c|c|c|c|c|}
\hline \multirow[t]{2}{*}{ Patient and tumour characteristics } & \multicolumn{2}{|c|}{ Responders } & \multicolumn{2}{|c|}{ Non-responders } & \multirow[t]{2}{*}{$\mathrm{P}^{\mathrm{a}}$} \\
\hline & $N=408$ & $\%$ & $N=592$ & $\%$ & \\
\hline \multicolumn{6}{|l|}{ Age } \\
\hline$<50$ & 82 & $20 \%$ & 147 & $25 \%$ & 0.010 \\
\hline $50-59$ & 127 & $31 \%$ & 157 & $27 \%$ & \\
\hline $60-69$ & 128 & $31 \%$ & 150 & $25 \%$ & \\
\hline $70+$ & 71 & $17 \%$ & 138 & $23 \%$ & \\
\hline \multicolumn{6}{|l|}{ Year of diagnosis } \\
\hline 2012 & 55 & $13 \%$ & 116 & $20 \%$ & 0.120 \\
\hline 2013 & 93 & $23 \%$ & 115 & $19 \%$ & \\
\hline 2014 & 86 & $21 \%$ & 116 & $20 \%$ & \\
\hline 2015 & 89 & $22 \%$ & 118 & $20 \%$ & \\
\hline 2016 & 85 & $21 \%$ & 127 & $21 \%$ & \\
\hline \multicolumn{6}{|l|}{ Type of surgery* } \\
\hline Breast conserving & 162 & $40 \%$ & 223 & $38 \%$ & 0.769 \\
\hline Amputation & 240 & $59 \%$ & 360 & $61 \%$ & \\
\hline \multicolumn{6}{|l|}{ Stage } \\
\hline $1 \mathrm{~A}$ & 175 & $43 \%$ & 265 & $45 \%$ & 0.205 \\
\hline $1 \mathrm{~B}$ & 13 & $3 \%$ & 24 & $4 \%$ & \\
\hline $2 \mathrm{~A}$ & 112 & $27 \%$ & 173 & $29 \%$ & \\
\hline $2 B$ & 64 & $16 \%$ & 59 & $10 \%$ & \\
\hline $3 \mathrm{~A}$ & 24 & $6 \%$ & 41 & $7 \%$ & \\
\hline $3 B$ & 9 & $2 \%$ & 10 & $2 \%$ & \\
\hline $3 C$ & 11 & $3 \%$ & 20 & $3 \%$ & \\
\hline \multicolumn{6}{|l|}{ Type of hospital $^{b}$} \\
\hline General hospital & 178 & $44 \%$ & 274 & $46 \%$ & 0.277 \\
\hline Teaching ("top-clinical”) hospital & 221 & $54 \%$ & 297 & $50 \%$ & \\
\hline Academic hospital & 9 & $2 \%$ & 21 & $4 \%$ & \\
\hline
\end{tabular}

a Chi-square tested.

${ }^{b}$ Hospitals were categorised as either general, teaching, or academic hospitals.

*do not add up due to missing values 
Supplementary Table 2: Mean quality of life domain scores per year since diagnosis

\begin{tabular}{|c|c|c|c|c|c|c|c|}
\hline \multirow{3}{*}{$\begin{array}{l}\text { HRQoL } \\
\text { domains }\end{array}$} & \multicolumn{7}{|c|}{ Time since diagnosis } \\
\hline & \multicolumn{2}{|l|}{$<2$} & \multicolumn{2}{|l|}{$2-4$} & \multicolumn{2}{|l|}{$4+$} & \multirow[b]{2}{*}{$\mathrm{p}$} \\
\hline & mean & SD & mean & SD & mean & SD & \\
\hline Global health status & 77.9 & 16.6 & 75.7 & 27.1 & 76.0 & 8.3 & 0.616 \\
\hline Physical functioning & 84.0 & 14.9 & 83.9 & 16.2 & 82.3 & 17.5 & 0.641 \\
\hline Role functioning & 81.1 & 24.5 & 79.3 & 22.4 & 81.0 & 24.4 & 0.777 \\
\hline Emotional functioning & 83.3 & 16.9 & 83.1 & 19.5 & 82.4 & 22.1 & 0.927 \\
\hline Cognitive functioning & 80.3 & 22.4 & 80.4 & 22.1 & 81.0 & 21.5 & 0.966 \\
\hline Social functioning & 84.6 & 23.1 & 84.2 & 22.0 & 88.1 & 20.1 & 0.248 \\
\hline
\end{tabular}

Supplementary Table 3: Mean quality of life domain scores per number of symptom categories reported

\begin{tabular}{|c|c|c|c|c|c|c|c|c|c|}
\hline \multicolumn{10}{|c|}{ Number of symptom categories* } \\
\hline & 0 & & $1-4$ & & $5-8$ & & 8-11 & & \\
\hline \multirow[t]{2}{*}{ Group size $n,(\%)$} & $12(3)$ & & $142(37)$ & & $193(50)$ & & $42(11)$ & & \\
\hline & mean & SD & mean & SD & mean & SD & mean & SD & $\mathrm{p}$ \\
\hline $\begin{array}{l}\text { Global health } \\
\text { status }\end{array}$ & 91.7 & 8.7 & 84.0 & 13.5 & 71.3 & 16.3 & 64.9 & 19.8 & $<0.001$ \\
\hline $\begin{array}{l}\text { Physical } \\
\text { functioning }\end{array}$ & 96.7 & 5.3 & 89.9 & 10.7 & 79.2 & 18.0 & 74.5 & 16.0 & $<0.001$ \\
\hline Role functioning & 97.2 & 9.6 & 91.0 & 15.4 & 73.4 & 24.5 & 66.3 & 27.4 & $<0.001$ \\
\hline $\begin{array}{l}\text { Emotional } \\
\text { functioning }\end{array}$ & 97.9 & 5.2 & 92.3 & 12.1 & 77.8 & 20.7 & 67.0 & 23.5 & $<0.001$ \\
\hline $\begin{array}{l}\text { Cognitive } \\
\text { functioning }\end{array}$ & 95.8 & 7.5 & 92.7 & 13.0 & 73.6 & 22.1 & 63.1 & 24.0 & $<0.001$ \\
\hline $\begin{array}{l}\text { Social } \\
\text { functioning }\end{array}$ & 100.0 & 0.0 & 94.6 & 12.1 & 80.6 & 22.9 & 71.8 & 29.3 & $<0.001$ \\
\hline
\end{tabular}

* Categories of health symptoms as described in Table 2 (11 catagories in total). Health symptoms were categorized in ten categories based on organ system 


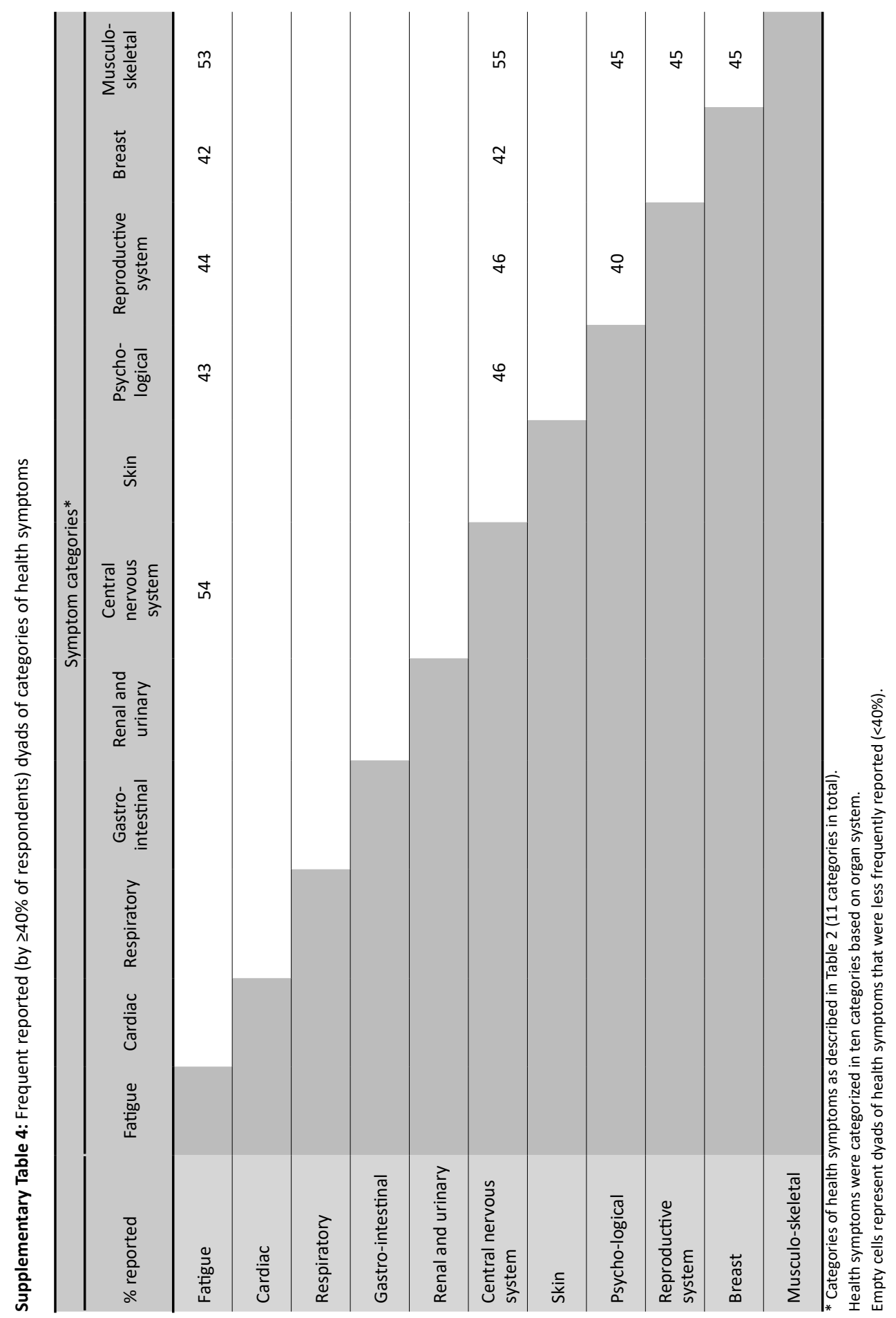


Supplementary Table 5: multivariate linear regression for the effect of reported health problems on EORTC-QLQ-C30 quality of life domains, including the corrections for covariates

\begin{tabular}{|c|c|c|c|c|c|c|c|}
\hline \multicolumn{2}{|c|}{$\begin{array}{l}\text { Quality of life domain } \\
(\beta, 95 \%-C I)\end{array}$} & \multirow{2}{*}{$\begin{array}{c}\begin{array}{c}\text { Global } \\
\text { health } \\
\text { status }\end{array} \\
\text { Ref }\end{array}$} & \multirow{2}{*}{$\begin{array}{c}\text { Physical } \\
\text { functioning } \\
\text { ref }\end{array}$} & \multirow{2}{*}{$\begin{array}{c}\text { Role } \\
\text { functioning } \\
\text { ref }\end{array}$} & \multirow{2}{*}{$\begin{array}{c}\begin{array}{c}\text { Emotional } \\
\text { functioning }\end{array} \\
\text { ref }\end{array}$} & \multirow{2}{*}{$\begin{array}{c}\begin{array}{c}\text { Cognitive } \\
\text { functioning }\end{array} \\
\text { ref }\end{array}$} & \multirow{2}{*}{$\begin{array}{c}\text { Social } \\
\text { functioning } \\
\text { ref }\end{array}$} \\
\hline \multirow{4}{*}{ 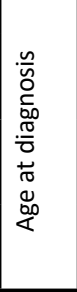 } & $<50$ & & & & & & \\
\hline & $50-59$ & & $\begin{array}{c}-0.8 \\
(-5.1 ; 3.6)\end{array}$ & & & $\begin{array}{c}3.0 \\
(-3.0 ; 9.1)\end{array}$ & $\begin{array}{c}5.5 \\
(-1.0 ; 12.0\end{array}$ \\
\hline & $60-69$ & & $\begin{array}{c}-6.8^{*} \\
(-11.0 ;-2.5)\end{array}$ & & & $\begin{array}{c}7.4^{*} \\
(1.5 ; 13.4)\end{array}$ & $\begin{array}{c}6.6 \\
(0.1 ; 13.0)\end{array}$ \\
\hline & $70+$ & & $\begin{array}{c}-13.5^{*} \\
(-18.1 ;-8.9)\end{array}$ & & & $\begin{array}{c}7.8^{*} \\
(1.4 ; 14.3)\end{array}$ & $\begin{array}{c}0.6 \\
(-6.6 ; 7.8)\end{array}$ \\
\hline \multirow{3}{*}{ 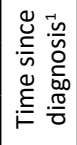 } & $<2$ & Ref & ref & ref & ref & ref & ref \\
\hline & $2-4$ & & & & & & \\
\hline & $>4$ & & & & & & \\
\hline \multirow{3}{*}{ 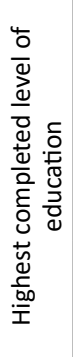 } & $\begin{array}{l}\text { Secondary } \\
\text { education or } \\
\text { lower }\end{array}$ & Ref & ref & ref & ref & ref & ref \\
\hline & $\begin{array}{l}\text { Medium } \\
\text { vocational } \\
\text { training (MBO) }\end{array}$ & & & & & & $\begin{array}{c}-2.0 \\
(7.2 ; 3.2)\end{array}$ \\
\hline & $\begin{array}{l}\text { High vocational } \\
\text { training (HBO/ } \\
\text { university) }\end{array}$ & & & & & & $\begin{array}{c}-5.6^{*} \\
(12.3 ;-0.8)\end{array}$ \\
\hline $\begin{array}{l}\text { 윻 } \\
\text { हे } \\
\text { Oे }\end{array}$ & Yes & $\begin{array}{c}-5.5^{*} \\
(-8.6 ;-2.4)\end{array}$ & $\begin{array}{c}-6.1^{*} \\
(-9.0 ;-3.3)\end{array}$ & $\begin{array}{c}-8.5^{*} \\
(12.9 ;-4.1)\end{array}$ & & & \\
\hline$\stackrel{\mathscr{c}}{\underline{\underline{m}}}$ & Yes & $\begin{array}{c}7.7^{*} \\
(2.3 ; 13.0)\end{array}$ & & & $\begin{array}{c}\mathbf{8 . 4} * \\
(2.3 ; 14.5)\end{array}$ & & \\
\hline \multirow{5}{*}{ 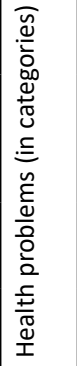 } & $\begin{array}{l}\text { Fatigue/ } \\
\text { endurance }\end{array}$ & $\begin{array}{c}-9.4 \\
(-13.0 ;-5.8)\end{array}$ & $\begin{array}{c}-8.2 \\
(-11.2 ;-3.3)\end{array}$ & $\begin{array}{c}-14.1 \\
(-18.9 ;-9.3)\end{array}$ & & & $\begin{array}{c}-6.0 \\
(-10.2 ;-1.7)\end{array}$ \\
\hline & Cardiac & $\begin{array}{c}-4.4 \\
(-8.3 ;-0.5)\end{array}$ & & & $\begin{array}{c}-5.5 \\
(-9.9 ;-1.0)\end{array}$ & $\begin{array}{c}-5.7 \\
(-10.5 ;-0.8)\end{array}$ & \\
\hline & Respiratory & & & & & & \\
\hline & Gastrointestinal & $\begin{array}{c}-3.4 \\
(-6.8 ;-0.1)\end{array}$ & $\begin{array}{c}-5.3 \\
(-8.2 ;-2.4)\end{array}$ & $\begin{array}{c}-7.8 \\
(-12.3 ;-3.3)\end{array}$ & & $\begin{array}{c}-4.3 \\
(-8.5 ;-0.1)\end{array}$ & \\
\hline & $\begin{array}{l}\text { Renal and } \\
\text { urinary }\end{array}$ & & & & & $\begin{array}{c}-9.7 \\
(-16.5 ;-2.8)\end{array}$ & \\
\hline
\end{tabular}

Supplementary table 5 continues on next page 
Continuation of supplementary table 5

\begin{tabular}{|c|c|c|c|c|c|c|c|}
\hline \multicolumn{2}{|c|}{$\begin{array}{l}\text { Quality of life domain } \\
(\beta, 95 \%-\mathrm{Cl})\end{array}$} & \multirow{2}{*}{$\begin{array}{c}\begin{array}{c}\text { Global } \\
\text { health } \\
\text { status }\end{array} \\
\begin{array}{c}-5.5 \\
(-9.3 ;-1.7)\end{array}\end{array}$} & \multirow[t]{2}{*}{$\begin{array}{c}\text { Physical } \\
\text { functioning }\end{array}$} & \multirow[t]{2}{*}{$\begin{array}{c}\text { Role } \\
\text { functioning }\end{array}$} & \multirow{2}{*}{$\begin{array}{c}\begin{array}{c}\text { Emotional } \\
\text { functioning }\end{array} \\
\begin{array}{c}-8.3 \\
(-12.5 ;-4.1)\end{array}\end{array}$} & \multirow{2}{*}{$\begin{array}{c}\begin{array}{c}\text { Cognitive } \\
\text { functioning }\end{array} \\
\begin{array}{c}-16.2 \\
(-20.8 ;-11.6)\end{array}\end{array}$} & \multirow{2}{*}{$\begin{array}{c}\begin{array}{c}\text { Social } \\
\text { functioning }\end{array} \\
\begin{array}{c}-6.1 \\
(-11.2 ;-0.9)\end{array}\end{array}$} \\
\hline \multirow{6}{*}{ 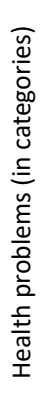 } & $\begin{array}{l}\text { Central nervous } \\
\text { system }\end{array}$ & & & & & & \\
\hline & Skin & & & & & & \\
\hline & Psychological & & & & $\begin{array}{c}-12.7 \\
(-12.7 ;-8.8)\end{array}$ & $\begin{array}{c}-5.2 \\
(-9.3 ;-0.9)\end{array}$ & $\begin{array}{c}-6.2 \\
(-10.8 ;-1.7)\end{array}$ \\
\hline & $\begin{array}{l}\text { Reproductive } \\
\text { system }\end{array}$ & & & & & & \\
\hline & Breast & & & & & & \\
\hline & Musculoskeletal & $\begin{array}{c}-4.2 \\
(-8.0 ;-0.5)\end{array}$ & $\begin{array}{c}-7.6 \\
(-11.0 ;-4.3)\end{array}$ & $\begin{array}{c}-9.5 \\
(-14.7 ;-4.3)\end{array}$ & $\begin{array}{c}-5.0 \\
(-9.1 ;-0.8)\end{array}$ & & $\begin{array}{c}-6.3 \\
(-11.4 ;-1.2)\end{array}$ \\
\hline
\end{tabular}

$\mathrm{Cl}$ : confidence interval; IBR: immediate breast reconstruction; comorb: comorbid diseases at time of questionnaire Factors that were significant in univariate testing $(p<0.10)$ were included in multivariate testing. Cells are empty when factors were not significant in univariate testing and thus not included in multivariate testing, or were excluded through backward selection in the multivariate analyses.

Reference categories for health problems were patients who not reported health problems in this category.

Corrected for age at diagnosis, presence of comorbid diseases, highest completed education at time of diagnosis, and breast reconstruction.

* significant in multivariate testing (level of significance in multivariate testing: 0.05 ).

1 in years 



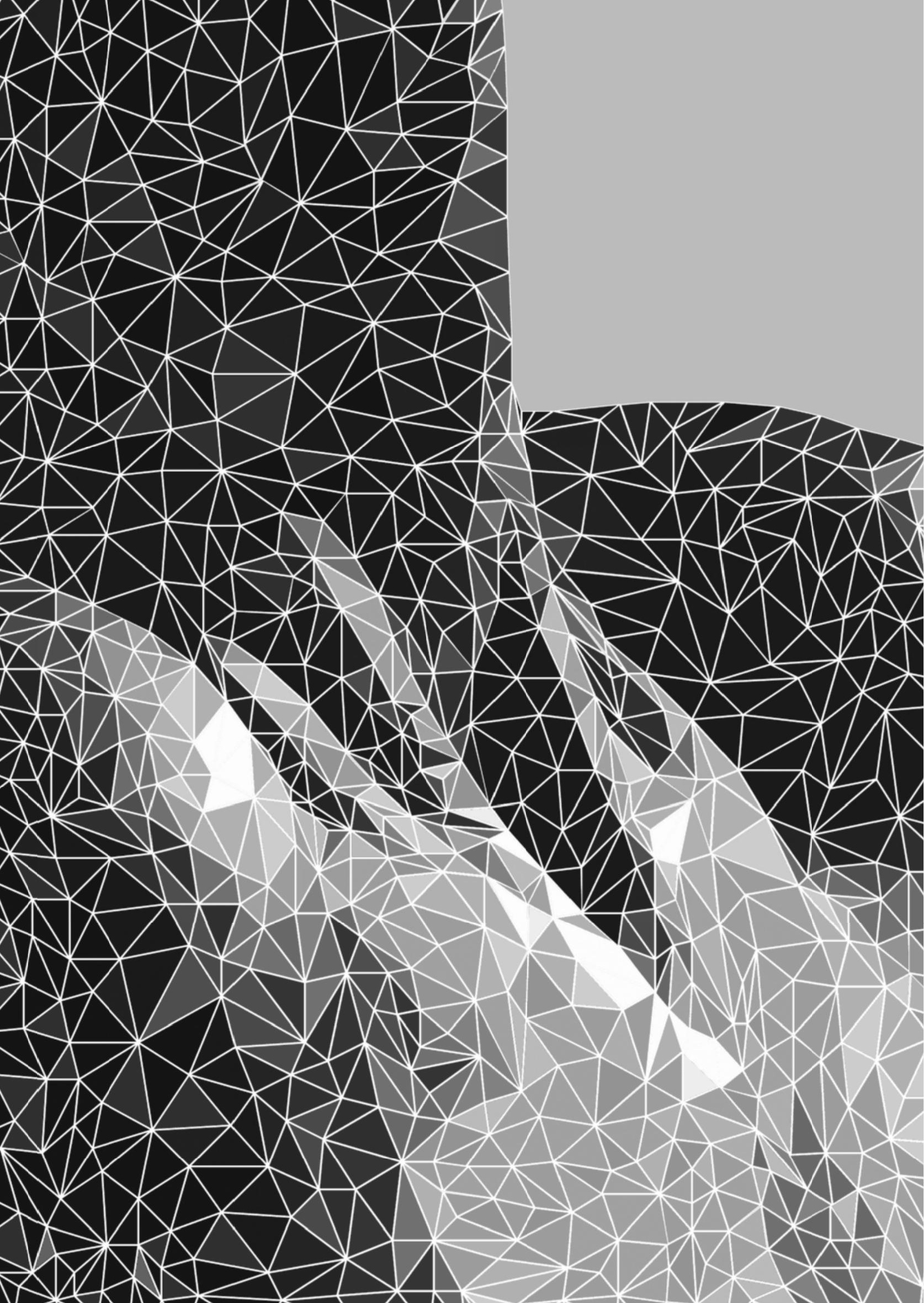




\section{CHAPTER 7}

\section{OPPORTUNITIES FOR PERSONALISED FOLLOW-UP CARE AMONG PATIENTS WITH BREAST CANCER: A SCOPING REVIEW TO IDENTIFY PREFERENCE SENSITIVE DECISIONS}

K.M. de Ligt L.S.E. van Egdom L.B. Koppert

S. Siesling J.A. van Til 



\section{Abstract}

Introduction:

Current follow-up arrangements for breast cancer do not optimally meet the needs of individual patients. We therefore reviewed the evidence on preferences and patient involvement in decisions about breast cancer follow-up to explore the potential for personalised care.

\section{Methods:}

Studies published between 2008 and 2017 were extracted from MEDLINE, PsycINFO, and Embase. We then identified decision categories related to content and form of follow-up. Criteria for preference sensitiveness and patient-involvement were compiled and applied to determine the extent to which decisions were sensitive to patient preferences and patients were involved.

\section{Results:}

Forty-one studies were included in the full-text analysis. Four decision categories were identified: 'surveillance for recurrent/secondary breast cancer; consultations for physical and psychosocial effects; recurrence-risk reduction by anti-hormonal treatment, and improving quality of life after breast cancer'. There was little evidence that physicians treated decisions about anti-hormonal treatment, menopausal symptoms, and follow-up consultations as sensitive to patient preferences. Decisions about breast reconstruction were considered as very sensitive to patient preferences, and patients were usually involved.

\section{Conclusion:}

Patients are currently not involved in all decisions that affect them during follow-up, indicating a need for improvements. Personalised follow-up care could improve resource allocation and the value of care for patients. 


\section{Background}

Breast cancer is the most common form of cancer among women worldwide [1]. The five-year relative survival for early stage breast cancer is high, with rates exceeding $96 \%$ for stage I and $86 \%$ for stage II disease [2,3]. International guidelines state that the goals of breast cancer follow-up care are to detect recurrent disease or new malignancies at an early stage, and to inform and counsel patients about the physical and psychosocial (late) effects of therapy [4-7]. Schemes for detecting recurrences often comprise annual physical and mammographic examinations for at least five years, depending on the patient's age, genetic predisposition, and/or tumour characteristics. Consultations that seek to detect physical and psychosocial effects are often linked to the visits for recurrence detection, and are most frequently planned during the first year of follow-up $[4,6]$.

At present, arrangements for follow-up suboptimally meet the needs of patients with breast cancer, and there is concurrently a growing demand for personalised care planning within cancer follow-up care [8-11]. Such personalised follow-up care could be based on the patient's individual risk of recurrence for the length and/or frequency of surveillance $[12,13]$, or on the type of treatment, and therefore, the management of treatment-induced (late) effects and complaints $[4,6]$. Moreover, cancer survivors might experience very different psychosocial consequences after the disease and treatment, including fear of recurrence, sleeping difficulties, cognitive issues, fatigue, and sexual issues [14]. Each of these effects require a personalized follow-up strategy. Patient-preferences about the preferred form and content of the follow-up care have been reported in previous studies $[15,16]$.

Since the advent of value-based healthcare, there have been ongoing efforts to improve care quality by adding value throughout an individual patient's journey from diagnosis, through treatment, and to follow-up care [17]. A way to meet this goal of personalised care is to include patients and their preferences in the decision-making process. For example, in the shared decisionmaking (SDM) process, decisions are based on both the best available (medical) evidence and the patients' needs and values. Preference sensitive care involves making treatment decisions with significant trade-offs that should reflect a patient's personal values and preferences. Besides, only when patients have enough information to make an informed choice, a decision can be made [18]. This means that the quality of this SDM process might affect the eventual effect on the value of care, in terms of outcomes, costs, and organizational effort [19].

In the present study, we hypothesised that decisions about breast cancer follow-up are sensitive to patient preferences, and that it is an option to include SDM in the follow-up care of these patients. Thus, we aimed to discover the potential for personalising follow-up care among patients with breast cancer by exploring the evidence on preferences for, and patient involvement in, decisions about breast cancer follow-up care.

\section{Methods}

The review was registered in PROSPERO (reference No.: CRD42018082501) [20]. 


\section{Search strategy}

Three research questions were posed: (1) 'what decisions are made during follow-up about content or form of follow-up care for breast cancer survivors?'; (2) 'to what extent are these decisions sensitive to patient preferences?', and (3) 'to what extent and how are patients with breast cancer involved in making these decisions?'. The literature was searched separately for each question, between 18th July and 25th September 2017, in the MEDLINE (accessed through PubMed), PsycINFO (accessed through Ovid), and Embase databases (Table 1). We included any study that discussed decisions made or interventions applied during follow-up for breast cancer, provided it was written in English and published in the last 10 years (2008-2017). The time restriction was set because breast cancer care and treatment have changed significantly over previous decades. The follow-up period was defined as the time period after surgery for breast cancer.

After removing duplicates, study titles and abstracts were screened by two independent screeners (KdL and LE). Studies were excluded if they did not include patients with breast cancer, did not discuss follow-up, did not describe actual decision-making, or did not describe the patients' roles in decision-making. Studies were also excluded if they included patients receiving palliative treatment. Full texts were retrieved for the remaining studies. Those without full text articles were excluded after attempt to contact the corresponding authors to access the text. EndNote [21] was used to manage all search results.

Table 1: search strategy per research question* for MEDLINE (accessed through PubMed), PsycINFO (accessed through Ovid), and Embase

\begin{tabular}{|c|c|c|c|c|c|c|}
\hline \multirow[t]{2}{*}{ Search words } & \multirow{2}{*}{$\begin{array}{l}\text { Databases } \\
\text { MEDLINE (PubMed) }\end{array}$} & \multicolumn{5}{|l|}{ Research question* } \\
\hline & & PsychINFO (Ovid) & Embase & 1 & 2 & 3 \\
\hline Breast cancer & $\begin{array}{l}\text { (("breast"[MeSH Terms] OR } \\
\text { "breast"[All Fields]) AND } \\
\text { ("neoplasms"[MeSH Terms] } \\
\text { OR "neoplasms"[All Fields] } \\
\text { OR "cancer"[All Fields])) OR } \\
\text { ("neoplasms"[MeSH Terms] } \\
\text { OR "neoplasms"[All Fields] } \\
\text { OR "malignancy"[All Fields]) } \\
\text { OR ("tumour"[All Fields] OR } \\
\text { "neoplasms"[MeSH Terms] } \\
\text { OR "neoplasms"[All Fields] } \\
\text { OR "tumor"[All Fields]) OR } \\
\text { ("carcinoma"[MeSH Terms] } \\
\text { OR "carcinoma"[All Fields]) } \\
\text { OR "neoplasms"[MeSH } \\
\text { Terms] OR "neoplasms"[All } \\
\text { Fields] OR "neoplasm"[All } \\
\text { Fields] OR "mass"[All Fields] } \\
\text { OR Nodule[All Fields] OR } \\
\text { ("cysts"[MeSH Terms] } \\
\text { OR "cysts"[All Fields] OR } \\
\text { "cyst"[All Fields]) }\end{array}$ & $\begin{array}{l}\text { exp BREAST NEOPLASMS/ } \\
\text { OR (exp BREAST/ AND exp } \\
\text { NEOPLASMS/ ) OR breast } \\
\text { cancer.mp OR ((breast. } \\
\text { mp OR exp BREAST/ ) AND } \\
\text { (cancer.mp OR neoplasm*. } \\
\text { mp OR carcin*.mp OR } \\
\text { tumor*.mp OR tumour*.mp } \\
\text { OR metasta*.mp OR malig*. } \\
\text { mp)) }\end{array}$ & $\begin{array}{l}\text { breast cancer'/exp } \\
\text { OR (breast:ti,ab AND } \\
\text { carcinoma*:ti,ab) } \\
\text { OR (breast:ti,ab AND } \\
\text { cancer*:ti,ab) OR } \\
\text { (breast:ti,ab AND } \\
\text { neoplasm*:ti,ab) OR } \\
\text { (breast:ti,ab AND } \\
\text { tumour*:ti,ab) OR } \\
\text { (breast:ti,ab AND } \\
\text { tumor*:ti,ab) OR } \\
\text { (breast:ti,ab AND } \\
\text { metasta*:ti,ab) OR } \\
\text { (breast:ti,ab AND } \\
\text { malig*:ti,ab) OR } \\
\text { ('breast'/exp AND } \\
\text { (neoplas*:ti,ab OR } \\
\text { cancer*:ti,ab OR } \\
\text { carcin*:ti,ab OR } \\
\text { tumor*:ti,ab OR } \\
\text { tumour*:ti,ab OR } \\
\text { metasta*:ti,ab OR } \\
\text { malig*:ti,ab OR } \\
\text { 'neoplasm'/exp)) }\end{array}$ & $x$ & $x$ & $\mathrm{x}$ \\
\hline
\end{tabular}

Table 1 continues on next page 
Continuation of table 1

\begin{tabular}{|c|c|c|c|c|c|c|}
\hline Search words & Databases & Research question* & & & & \\
\hline & MEDLINE (PubMed) & PsychINFO (Ovid) & Embase & 1 & 2 & 3 \\
\hline Follow-up & $\begin{array}{l}\text { follow-up[All Fields] OR } \\
\text { ("aftercare"[MeSH Terms] } \\
\text { OR "aftercare"[All Fields] } \\
\text { OR ("after"[All Fields] AND } \\
\text { "treatment"[All Fields]) OR } \\
\text { "after treatment"[All Fields]) } \\
\text { OR "survival"[MeSH Terms] } \\
\text { OR "survival"[All Fields] OR } \\
\text { "survivorship"[All Fields] OR } \\
\text { (care[All Fields] AND plan[All } \\
\text { Fields]) OR care[All Fields] } \\
\text { OR surveillance [All Fields] }\end{array}$ & $\begin{array}{l}\text { follow-up.mp. OR } \\
\text { exp POSTTREATMENT } \\
\text { FOLLOWUP/ OR followup. } \\
\text { mp OR aftercare.mp } \\
\text { OR after-care.mp OR } \\
\text { exp Aftercare/ OR ((exp } \\
\text { PATIENTS/ or patient.mp) } \\
\text { AND (monitoring.mp. or } \\
\text { exp MONITORING/)) OR } \\
\text { after treatment.mp OR } \\
\text { exp Survivors/ OR survival. } \\
\text { mp OR survivorship.mp OR } \\
\text { exp Treatment Planning/ } \\
\text { OR care plan.mp OR } \\
\text { surveillance.mp }\end{array}$ & $\begin{array}{l}\text { follow up':ti,ab OR } \\
\text { 'aftercare':ti,ab } \\
\text { OR 'aftercare'/de } \\
\text { OR (after NEAR/1 } \\
\text { treatment):ti,ab } \\
\text { OR 'survival':ti,ab } \\
\text { OR 'survival'/de OR } \\
\text { 'survivorship'/de OR } \\
\text { 'survivorship':ti,ab } \\
\text { OR (care NEAR/1 } \\
\text { plan):ti,ab OR } \\
\text { 'surveillance'/de OR } \\
\text { 'surveillance' }\end{array}$ & $x$ & & $x$ \\
\hline $\begin{array}{l}\text { Decision- } \\
\text { making }\end{array}$ & $\begin{array}{l}\text { ("Decisions"[Journal] OR } \\
\text { "decisions"[All Fields]) } \\
\text { AND ("decision support } \\
\text { techniques"[MeSH Terms] } \\
\text { OR ("decision"[All Fields] } \\
\text { AND "support"[All Fields] } \\
\text { AND "techniques"[All } \\
\text { Fields]) OR "decision support } \\
\text { techniques"[All Fields] } \\
\text { OR ("decision"[All Fields] } \\
\text { AND "analysis"[All Fields]) } \\
\text { OR "decision analysis"[All } \\
\text { Fields]) }\end{array}$ & $\begin{array}{l}\text { decision-making.mp. or } \\
\text { exp Decision Making/ } \\
\text { OR ((support techniques. } \\
\text { mp) AND (decision. } \\
\text { mp)) OR ((support. } \\
\text { mp) AND (techniques. } \\
\text { mp)) OR decision } \\
\text { support techniques.mp } \\
\text { OR ((decision.mp) AND } \\
\text { (analysis.mp)) OR decision } \\
\text { analysis.mp }\end{array}$ & $\begin{array}{l}\text { decision making'/ } \\
\text { de OR 'decision } \\
\text { making':ti,ab } \\
\text { OR ('decision'/ } \\
\text { de OR decision } \\
\text { AND ('support'/ } \\
\text { de OR support) } \\
\text { AND techniques) } \\
\text { OR 'decision'/de } \\
\text { OR decision AND } \\
\text { ('analysis'/de OR } \\
\text { analysis) }\end{array}$ & $x$ & & \\
\hline $\begin{array}{l}\text { Preference- } \\
\text { sensitive } \\
\text { decisions }\end{array}$ & $\begin{array}{l}\text { preference[All Fields] AND } \\
\text { sensitive[All Fields] AND } \\
\text { ("Decisions"[Journal] OR } \\
\text { "decisions"[All Fields]) }\end{array}$ & preference-sensitive.mp & $\begin{array}{l}\text { preference } \\
\text { sensitive':ti,ab }\end{array}$ & & $x$ & \\
\hline $\begin{array}{l}\text { Shared } \\
\text { decision- } \\
\text { making }\end{array}$ & $\begin{array}{l}\text { decision making[MeSH } \\
\text { Terms] OR ("decision"[All } \\
\text { Fields] AND "making"[All } \\
\text { Fields]) OR "decision } \\
\text { making"[All Fields] OR } \\
\text { ("shared"[All Fields] AND } \\
\text { "decision"[All Fields] AND } \\
\text { "making"[All Fields]) OR } \\
\text { "shared decision making"[All } \\
\text { Fields] }\end{array}$ & $\begin{array}{l}\text { ((shared.mp) AND (decision- } \\
\text { making.mp or exp Decision } \\
\text { Making/)) }\end{array}$ & $\begin{array}{l}\text { shared decision } \\
\text { making'/de OR 'shared } \\
\text { decision making' }\end{array}$ & & & $x$ \\
\hline
\end{tabular}

* 1) What are the common complaints and issues that can occur for woman treated for breast cancer with curative intent for which decisions have to be made with regard to management within five years after curative treatment?; 2) To what extent are decisions with regard to the management of these complaints preference sensitive?; 3) To what extent and how are patients with breast cancer involved in making these follow-up-related decisions? 


\section{Quality assessment}

The quality of the included studies was assessed by the Critical Appraisal Skills Programme checklist, comprising criteria for qualitative studies, randomized controlled trails, cohort studies, and systematic reviews. Criteria could be scored with a positive or negative response; when criteria were not applicable or unknown/unable to be assessed, this was recorded as well [22]. First, we determined the study design for each included study, provided this was not already described in the study's method section. Studies were deemed of sufficient quality when half or more of the criteria could be scored positive, provided there was a clear aim or research question.

\section{Analyses}

First, we identified the decisions were made or could be made about content or form of follow-up care delivered to breast cancer patients. Second, criteria were compiled to determine whether decisions were sensitive to patient preferences and whether patients were involved in making the decisions. Third, these criteria, in turn, were used to assess the degree to which decisions were sensitive to patient preferences and the extent to which patients were involved in making these decisions.

Criteria for preference sensitiveness (PSO-5) were based on the definition by Van der Weijden et al. [23]. Decisions were considered preference-sensitive if the following criteria were met:

0 . There were multiple options available (PSO); and

1. Options had potential favourable and unfavourable outcomes, leading to an individual tradeoff (PS1); or

2. Options did not differ in terms of favourability of the outcomes, or (un)favourable outcomes were equally (un)desirable (PS2); or

3. There was insufficient evidence about favourable or unfavourable outcomes to determine the best option (PS3); or

4. The potential risks of an option were high, regardless the potential benefits of this option (PS4); or

5. The outcomes were highly dependent on patient cooperation, or the actions required for the preferred option had high impact on the patient's lifestyle (PS5).

Criteria for the extent of patient-involvement (SDM1-7) were based on the conditions set by Légaré et al. [18] and the components described by Coulter and Collins. [24]:

1. The decision was preference sensitive (SDM1); and

2. There was sufficient time to make a decision (SDM2); and/or

3. The patient was capable and sufficiently informed to make a decision (SDM3); and/or

4. There was a belief that SDM would lead to better patient outcomes (SDM4); and/or

5. The physician was motivated for SDM and clarified the options and preferences (SDM5); and/or

6. There was a belief that SDM will lead to better clinical outcomes (SDM6); and/or

7. There was a system for recording, communicating, and implementing the patient's preferences (SDM7). 


\section{Results}

Figure 1 summarises the selection process according to the PRISMA scheme. In total, 3077 records were screened after removing duplicates $(n=2539,28,1058$ per research question). After screening titles, abstracts, and full-texts, we finally included 41 studies.

Within the screened records, 'follow-up' often referred to the study design rather than the posttreatment period, and 'preference-sensitive' was used little or infrequently, only appearing as a key word in 21 records. Studies also generally described gaps in patient involvement rather than care that was already well-organized. Moreover, we excluded many studies $(n=2871)$ that could not be related to the SDM criteria because they did not describe decision-making about the content or form of follow-up care. Another 11 studies were excluded because the full texts were not available. These were mainly studies published as conference abstracts, dissertations, or books. Contact details were available for only five of the corresponding authors of these abstracts, and only one responded.

All included studies $(n=41)$ were rated as valuable in the quality assessment (Supplementary Table 2 ). Most studies employed a design with surveys $(n=11)$ or interviews $(n=16$; comprising focus groups, needs assessments, and semi-structured/directed/open-ended interviews. The surveybased studies included larger samples ( $n=5-41)$, whereas the interview-based studies included smaller groups ( $n=5-41$ ). Less common methods included studies of electronic health records $(n=1)$ and internet fora $(n=1)$. Randomized Controlled Trials (RCT) designs were used for studies about life style interventions $(n=2)$ and SDM-related tools about breast reconstruction $(n=3)$.

Table 2 summarizes the preference sensitive aspects (criterion PS) and aspects of patientinvolvement (criterion SDM) for each decision about the content or form of follow-up care. Decisions were classified into those concerning (1) 'surveillance for recurrent or secondary breast cancer'; (2) 'consultations for physical and psychosocial (late) effects'; (3) 'recurrence-risk reduction by anti-hormonal treatment'; and (4) 'improving quality of life after breast cancer'. Results are described in more detail below. Supplementary Table 1 summarizes the included studies. 


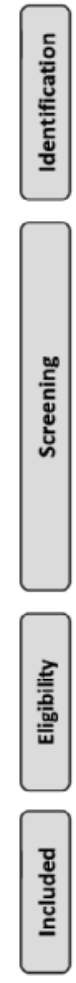

Records identified through database searching ( $n=3625)$

Per research question (Q): Q1 = 2539; Q2 = 28; Q3 = 1058

No records identified through other sources
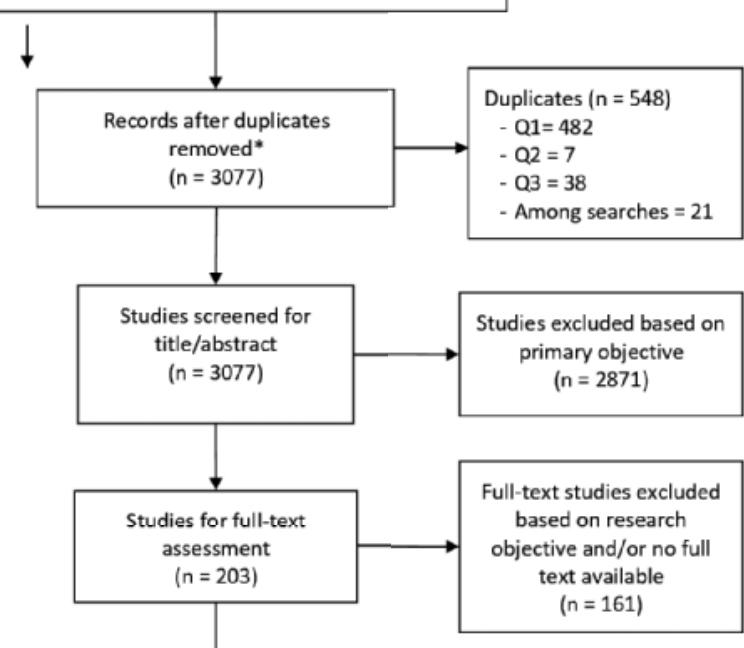

Studies included $* *$ ( $n=41)$

- Three literature searches were conducted (a search per research question), as shown in the identification box. Next, duplicates were removed from within each search, before being removed by cross-checking between the searches.

"- One duplicate was removed from the studies that were finally included.
"

Figure 1: PRISMA flow chart of study inclusion 


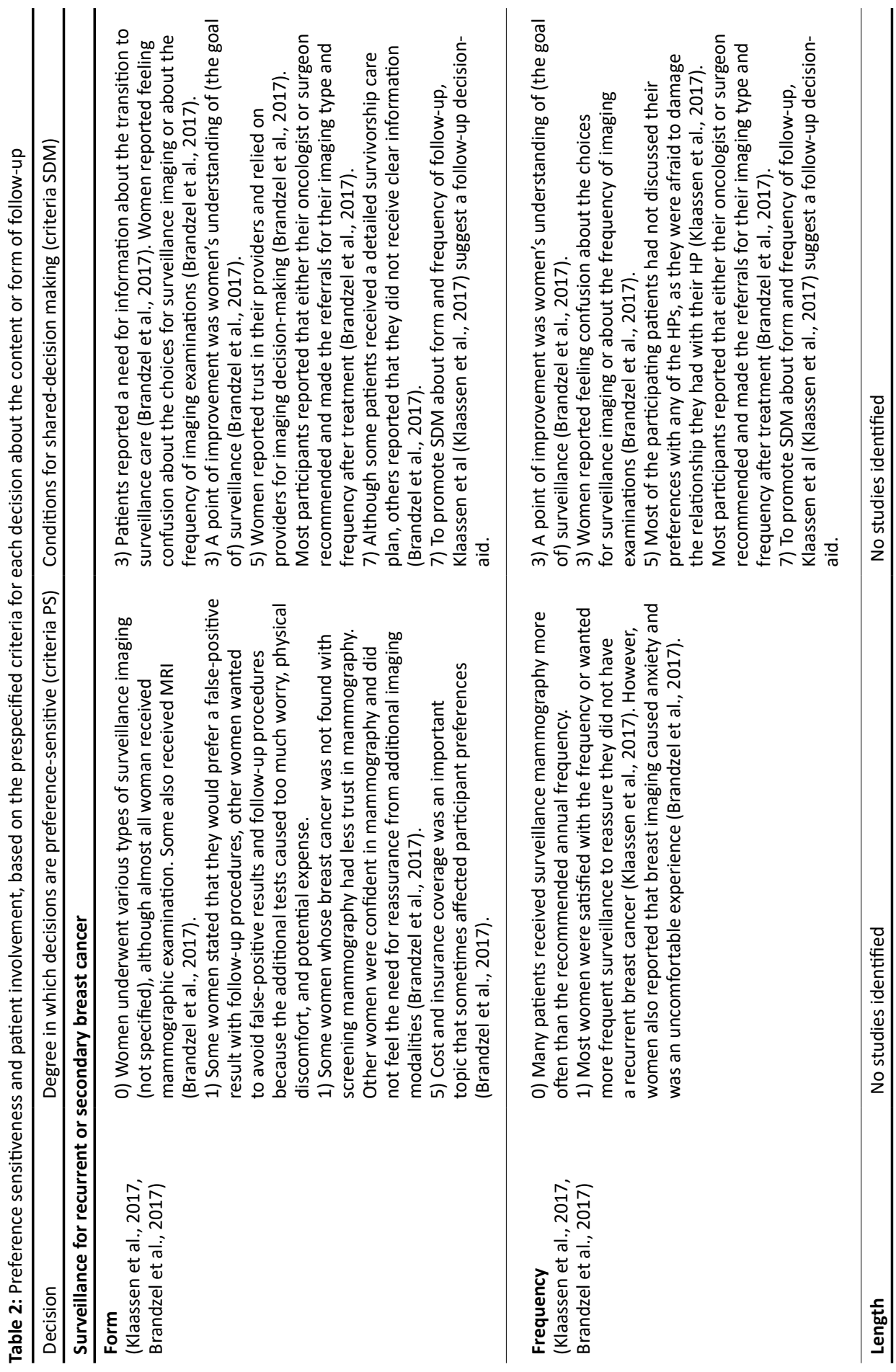




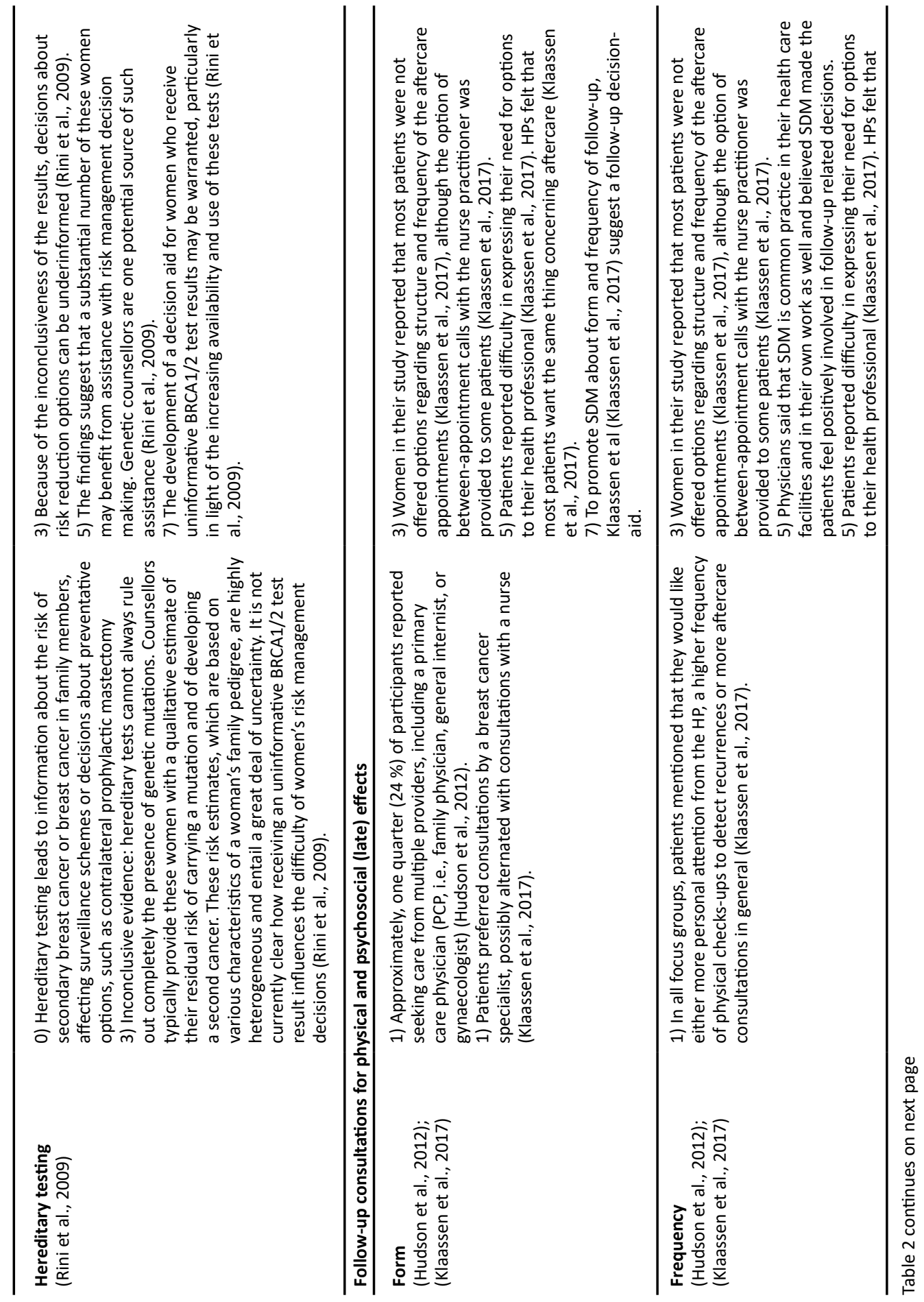




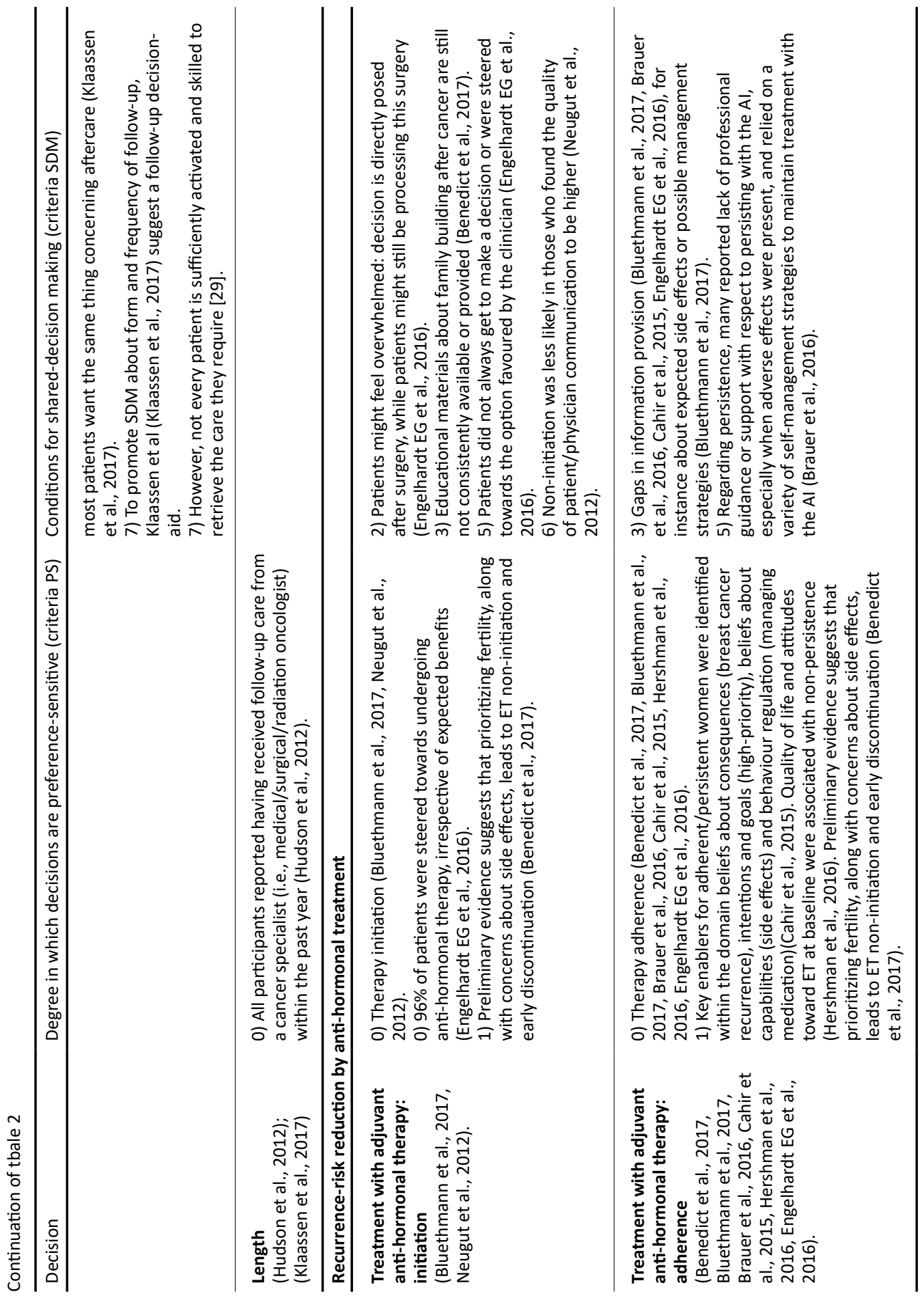




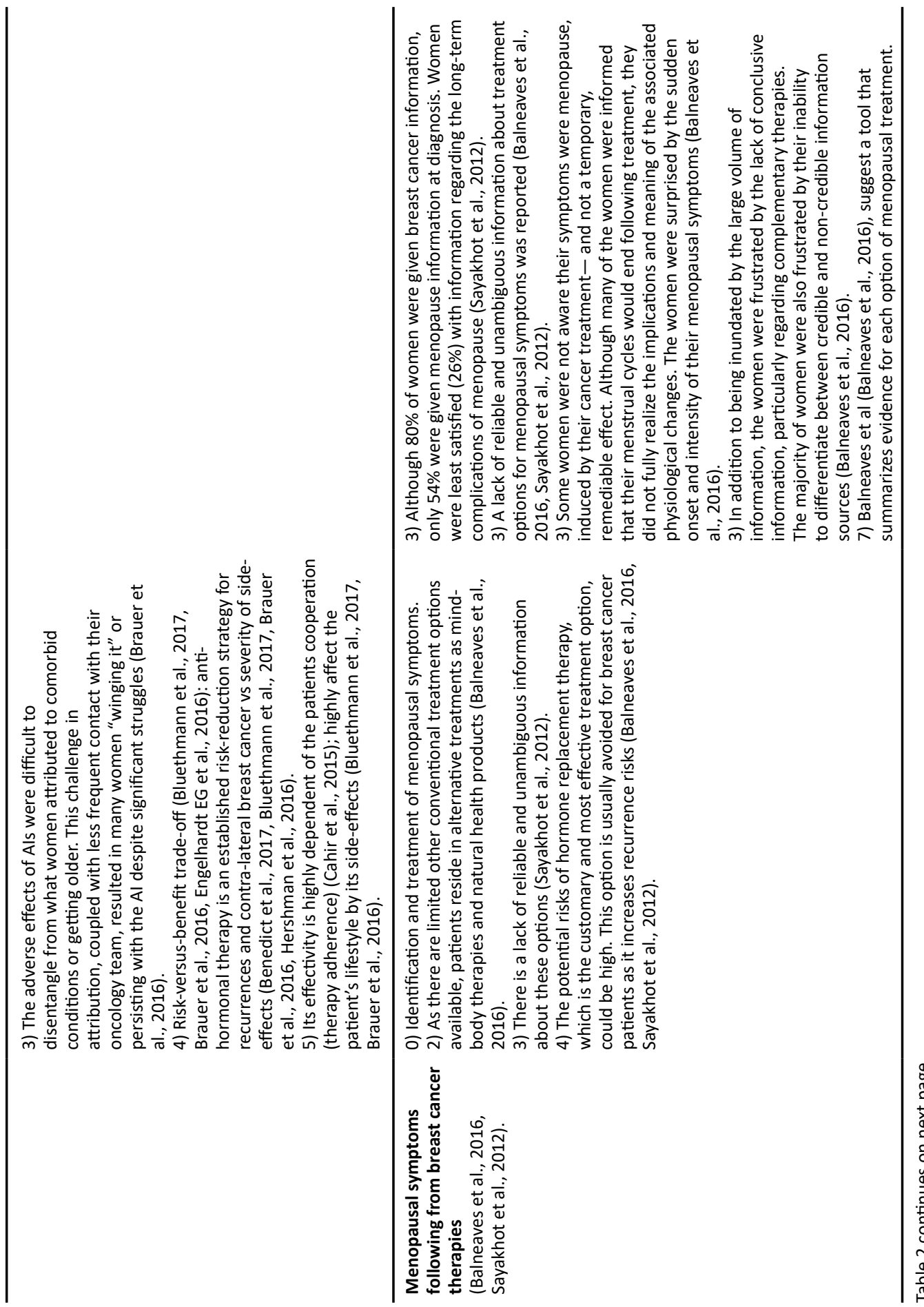




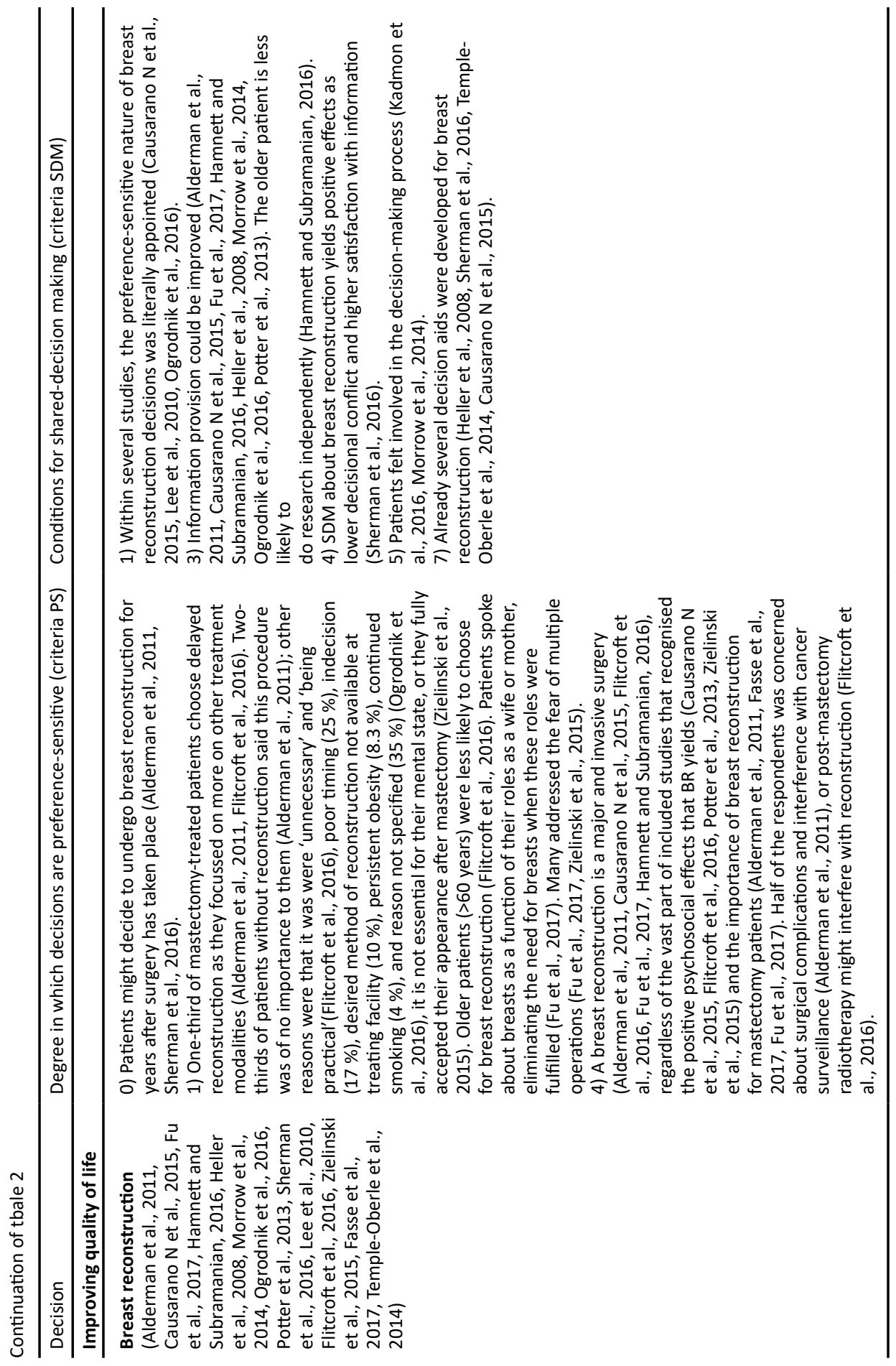




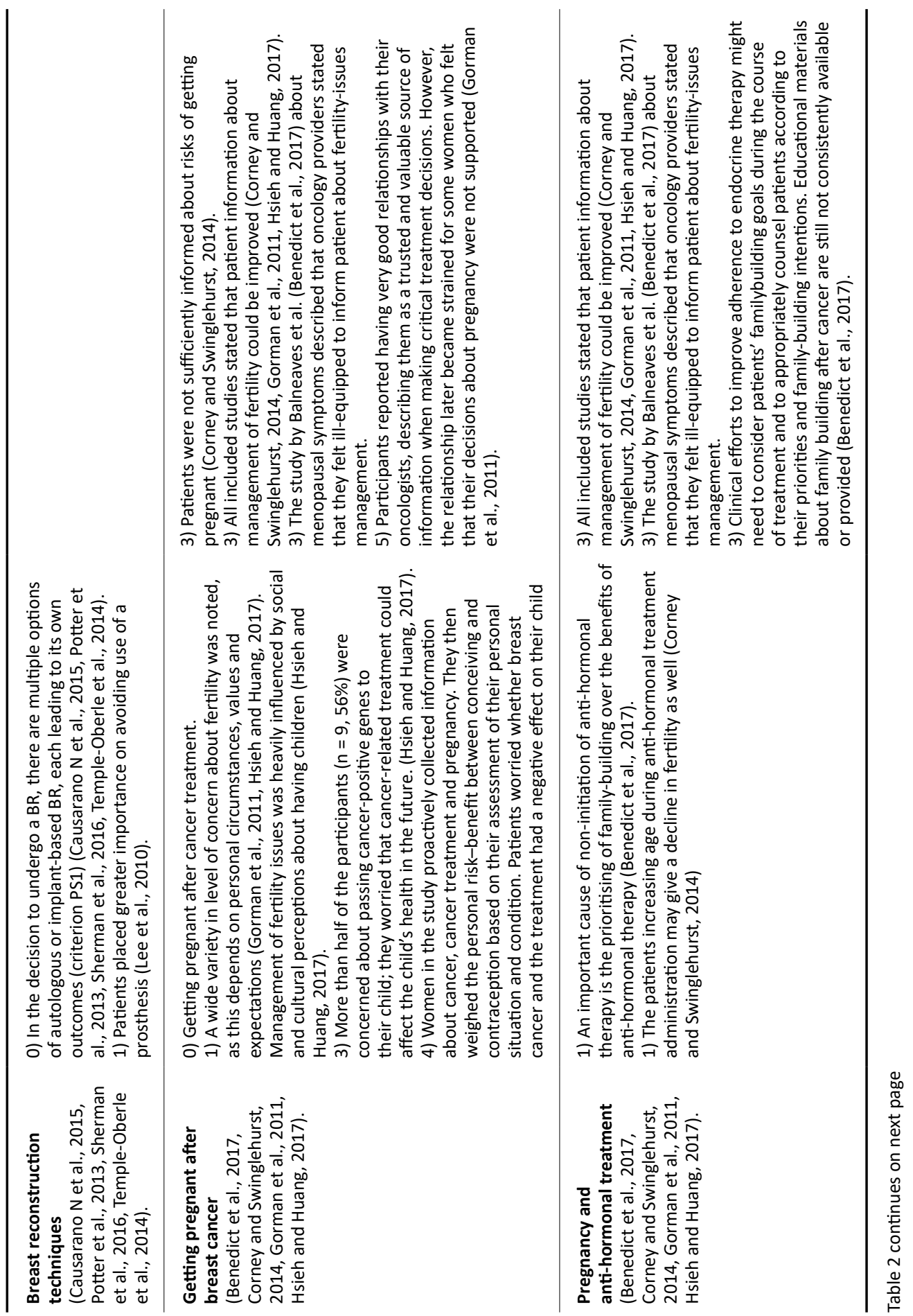




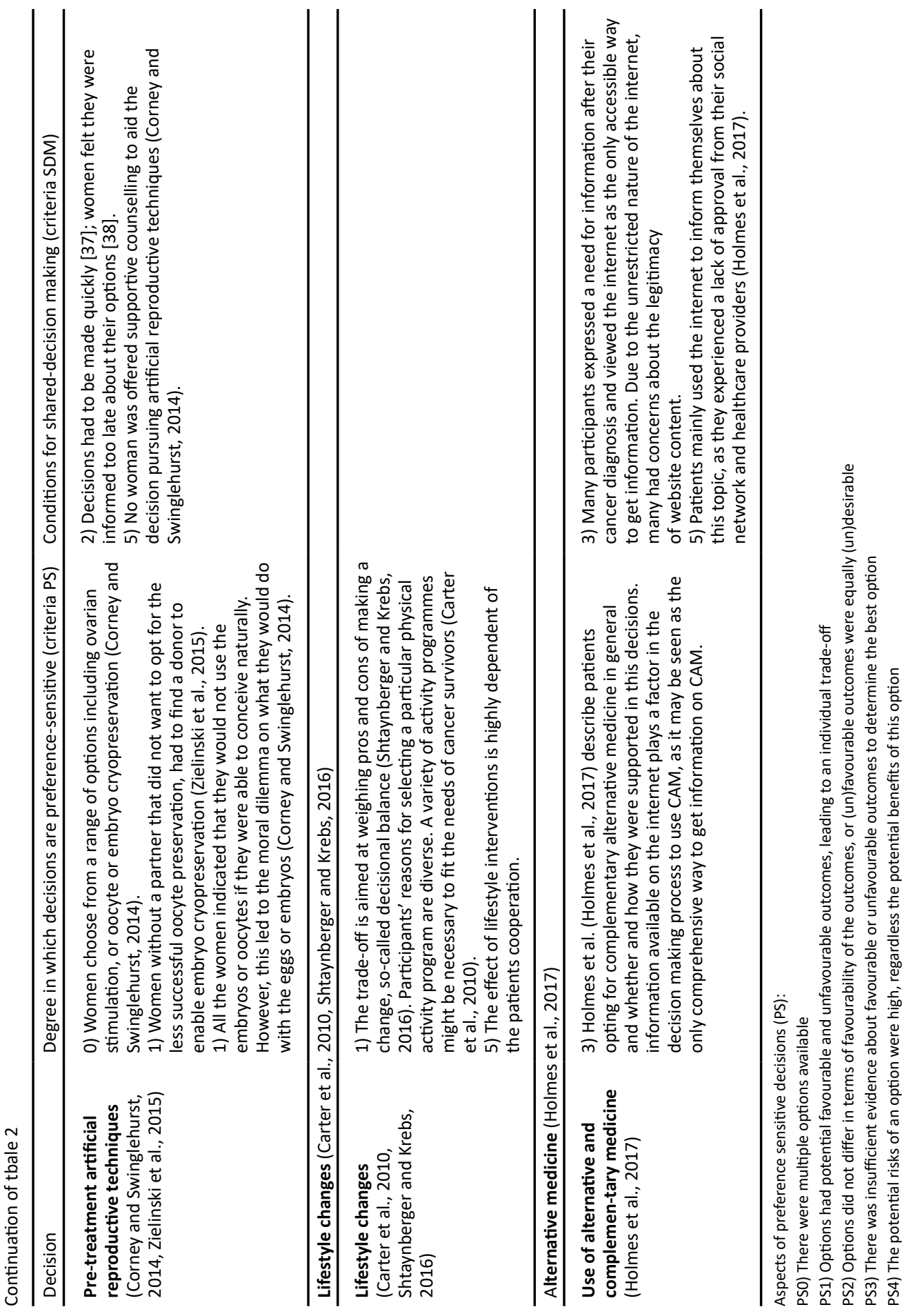




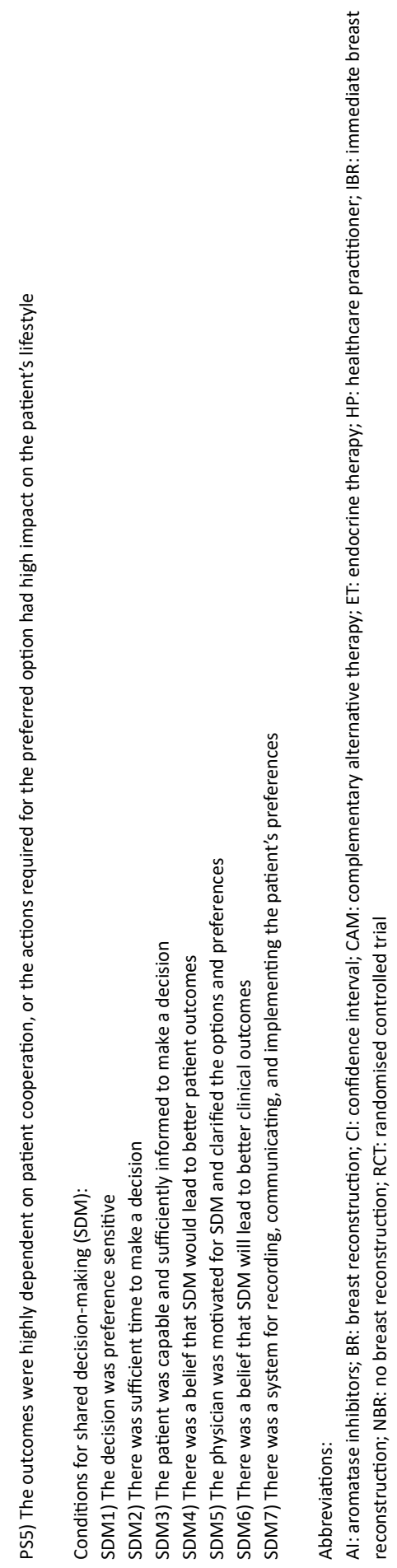




\section{(1) Surveillance for recurrent or secondary breast cancer}

Follow-up aims to detect recurrent disease or new associated malignancies at an early stage through surveillance imaging (mammography and/or MRI) and physical examination [4-7]. Two included studies discussed decisions about the form and frequency of surveillance imaging (PSO) $[25,26]$. Klaassen et al. assessed the needs of Dutch patients and physicians with regard to an aftercare decision-aid. Brandzel et al. then described the experiences and preferences for breast imaging among breast cancer survivors in the United States. The main form of surveillance tended to be mammography, though some also received MRI; however, the authors did not specify who received what type of surveillance imaging or the reasons for the differences. If their breast cancer initially was missed on mammography, patients sometimes lost trust in this method, and preferred other imaging modalities. Furthermore, many patients received surveillance mammography more often than the recommended annual frequency without clinical indication [26]. Patients preferred this higher frequency because it reassured them about the absence of recurrences $[25,26]$. However, breast imaging also caused anxiety and was considered uncomfortable for many patients [26], suggesting scope for a trade-off between burdens and benefits of surveillance imaging (PS1). Surveillance preferences were also affected by financial costs and insurance coverage [26], and therefore, the patient's willingness to bear these costs (PS5).

Little evidence was found for patient involvement in surveillance-related decisions. Brandzel et al. found that physicians typically determined the imaging type and frequency of surveillance (SDM5), despite the opposing preferences and trade-offs expressed by patients. The patient's understanding of the goal of surveillance could be improved here: patients felt confused about the options for the type of surveillance imaging and frequency of surveillance imaging, and expressed a need for information about the transition from treatment to surveillance care (SDM3). The aftercare decision-aid produced by Klaassen et al. provides an overview of follow-up options (SDM7), and could reduce information needs before initiating follow-up. Surveillance length was not discussed in the literature.

Hereditary testing is most often performed during breast cancer diagnosis and may be less relevant during follow-up [4]. However, Rini et al. [27] described hereditary testing in women with a history of breast cancer. Hereditary testing leads to information about the risk of secondary breast cancer, and/or risk of breast cancer or ovarian cancer in family members. This can affect surveillance schemes or preventative options, such as contralateral prophylactic mastectomy (PSO).

\section{(2) Consultations for physical and psychosocial (late) effects}

A second goal of follow-up is informing and counselling patients about the physical and psychosocial (late) effects of treatment [4-7]. Two studies described decision-making regarding the form, frequency, and length of follow-up consultations within follow-up care (PSO). Patients preferred more personal attention from their physician and a higher frequency of oncology-led aftercare than was offered (current situation not defined), which gave them more security about their health [25]. Regarding the length of follow-up consultation, all USA-based participants in a study by Hudson et al. had received follow-up care from a cancer specialist within the previous year, even though the time since their last active cancer treatment ranged from three to seventeen 
years; however, decisions about length were not discussed further [28]. Regarding the form of consultations, patients preferred consultations by a breast cancer specialist, possibly alternated with nurse consultations (PS1) [25]. Regardless of these preferences, patients were rarely offered options about the frequency, form, or length of consultations, indicating low patient-involvement.

By contrast, most physicians stated that SDM was common practice in their health care facilities and in their own work, and reported that using SDM made the patients feel positively involved in decisions related to follow-up (SDM5) [25]. Referral to other medical specialists or care providers during follow-up was not specifically described. However, $24 \%$ of patients sought care from multiple providers, including a primary care provider, general internist, or gynaecologist [28].

\section{(3) Recurrence risk reduction by anti-hormonal treatment}

Seven studies described treatment decisions about anti-hormonal therapy [29-35]. This consisted of tamoxifen or aromatase-inhibitor use to increase locoregional tumour control and survival, given for a minimum of five consecutive years, and continuing during follow-up [4]. Respectively, there were two and five studies on decisions regarding therapy initiation [30,35] and therapy adherence [29-32, 34]. Within the literature, therapy initiation was rarely regarded as a preference sensitive decision: one study described that $96 \%$ of patients were steered towards anti-hormonal therapy, irrespective of the expected benefit [33]; in another study, patients felt obliged to take the therapy (PS0) [30]. However, the decision about anti-hormonal therapy is not an one-off decision: four studies described that the decision to adhere to anti-hormonal therapy leads to patients making an ongoing risk-versus-benefit trade-off between the risk-reducing effect of treatment and the severity of treatment-induced side-effects (PS4) [29-32, 34]. Nonadherent patients in two studies felt unable to cope with side-effects that severely affected their lives (PS5) [30, 31]. Three studies reported that professional guidance or support from physicians for managing these side effects could be improved [29, 31,32]. Such guidance is important, because patients can better persevere with side effects if they have a high belief in their ability to manage and control their medication and side effects (PS1) [32]. However, four studies reported gaps in providing information about expected side effects $[29,30,33]$ or their management (SDM3) [30, 31].

Frequently reported effects of anti-hormonal therapy were menopausal symptoms and joint pain, with cognitive decline and cardiac distress also occurring, but less frequently [30]. Two studies specifically discussed the identification and treatment of treatment-induced menopausal symptoms (PSO) [36, 37], such as hot flashes, weight gain, loss of sexuality, and increased osteoporosis. Symptom treatment was considered a preference sensitive decision because hormone replacement therapy is the customary and most effective option, even though it increases the risk of recurrence and should be avoided in patients with breast cancer (PS4) [36, 37]. However, there are few alternatives (PS2), with these limited to various lifestyle changes, pharmaceutical options, and complementary treatments (e.g. mind-body therapies and natural health products) [36]. As both studies reported, a lack of reliable and unambiguous information about these options makes it difficult to select the best option (PS3). Concerning this dilemma, patients were frustrated by the lack of conclusive information, particularly about complementary therapies, and by an inability to differentiate between credible and non-credible information 
sources (SDM3). Balneaves et al. suggested using an SDM-tool that could summarise credible information about accepted options and thus facilitate decision-making (SDM7). Two-third of patients in this study still used complementary therapy to manage symptoms, despite the lack of information [36].

\section{(4) Improving quality of life after breast cancer treatment}

This topic was subdivided into three subtopics. Sixteen studies focused on delayed breast reconstruction, two on lifestyle changes, and four on getting pregnant after breast cancer.

Breast reconstruction yields positive psychosocial effects [38-41] and may contribute to the patients well-being after breast cancer. Although some, if not most decisions about breast reconstruction are made before surgical treatment, resulting in immediate breast reconstruction, some patients and/or clinicians delay the decision about breast reconstruction until after treatment. Patients must then first decide whether to undergo delayed breast reconstruction, and when they do, decide which reconstruction technique should be used (PSO). Decisions about delayed breast reconstruction can remain relevant years after tumour surgery $[42,43]$ and have been recognised as highly preference sensitive in three studies $[38,44,45]$. Furthermore, seven studies indicated that breast reconstruction yields positive psychosocial effects [38-41] and that it is an important option for patients who have undergone mastectomy [42, 46, 47]. In three studies, common reasons for opting to delay breast reconstruction rather than undergoing breast reconstruction were reported, and it was concluded that either patients wanted to focus on other treatment modalities first $[39,42]$, or that the desired technique was not available at their facility [45]. Patients generally refused breast reconstruction if they felt it was not important, urgent [42], or necessary, or feared undergoing further surgery [39]. Thus, apart from medical contra-indications, decisions about undergoing breast reconstruction were affected by its timing and individual decisions about trade-offs (PS1). Regardless of the potential for positive psychosocial effects [3841], risks of breast reconstruction can be high (PS4). Indeed, it is a major and invasive surgery [38, $39,42,47,48]$, and patients have reported concerns about surgical complications, and interference with cancer surveillance [42], or post-mastectomy radiotherapy [39]. There are also multiple options, such as autologous or implant-based breast reconstruction (PSO), with each associated with to different outcomes (PS1) [38, 40, 43, 49].

Current patient involvement in decisions about breast reconstruction appeared to be high: fifteen studies described elements of patient-involvement or SDM [38-48, 50-52], and patients in two studies specifically reported feeling involved in decision-making (SDM5) [51, 52]. SDM about breast reconstruction led to less conflict around decisions and to more satisfaction with the information provided (SDM4) [43]. By contrast, four studies reported that patients experienced decision-making uncertainty [41-43, 47] and eight studies recommended further improvement of information provision (SDM3) [38, 40, 42, 45, 47, 48, 50, 52]. This could be addressed by using one of four decision aids that have been developed (SDM7) $[38,43,49,50]$.

In younger patients, breast cancer treatment can interfere with the desire to have a family. Four studies described the decision to get pregnant after treatment for breast cancer [29, 53-55]. 
Although this decision may feel like a risk, there is consensus that pregnancy following breast cancer is safe [53]. Nevertheless, both patients and physicians have expressed concerns about the potential for pregnancy to increase recurrence risk in patients with hormone-sensitive breast cancer (PS4) [53-55]. Patients not only felt under informed (SDM3) [53], but also, patients worried whether breast cancer and its treatment would negatively affect the health of a future child (PS4) $[53,55]$. In general, there was a wide variety in the level of concern about fertility and getting pregnant. The importance of family building depended on personal circumstances, values, and expectations [53-55]. In a study of Chinese breast cancer survivors, social and cultural perceptions about having children were important motives (PS1) [55]. Although all three included studies described patient involvement in decisions about fertility management, it was also noted that the information provided could be improved (SDM3).

Anti-hormonal therapy may cause infertility in premenopausal patients. Those on anti-hormonal therapy may therefore have to wait to the end of the treatment period (i.e. five years), while may be accompanied by an age-related decline in fertility (PS1). In some patients, oncologists were willing to discuss the option of a reduced duration of anti-hormonal treatment [53]. Another study recognised the need to counsel patients about family-building periodically during anti-hormonal treatment [29]. Indeed, fertility counselling may remain important throughout follow-up because treatment-affected fertility may have negative psychosocial consequences $[54,55]$.

Chemotherapy treatment can also lead to reduced fertility. Therefore, patients should have the option to choose from a range of artificial reproductive techniques, including ovarian stimulation, and oocyte or embryo cryopreservation, before treatment (PSO) [53]. These decisions will also affect decision-making during follow up, for instance, patients who have opted for artificial reproductive techniques before treatment will have to decide on what to do with their preserved oocytes or embryos after treatment (PS0). All patients in a study by Corney and Swinglehurst [53] indicated that they would not use the embryos or oocytes if they were able to conceive naturally, leading to moral decision about what to do with the oocytes or embryos.

Quality of life improvements after cancer may be found by implementing lifestyle changes. Two RCTs described a lifestyle intervention and the reasons why patients did and did not participate (PSO) [56, 57]. Shtaynberger and Krebs [57] described how decisions about physical activities and fruit and vegetable intake were based on an individual weighing the pros and cons of making a change (the so-called decisional balance) (PS1). Carter et al. [56] described the reasons for cancer patients to participate in either of two physical activity programmes (walking or 'dragon boat' rowing') offered in their RCT. They reported that decisions were based on physical (health benefits), social (meeting new people, learning new skills), and practical (time investment, scheduling) considerations, but did not state whether the decision was discussed with a physician. 


\section{Discussion}

In this study, we aimed to assess the potential to personalise follow-up care for patients after breast cancer treatment, by exploring the evidence on patient preferences for, and patient involvement in decisions about follow-up care. We identified many decisions that needed to be made during follow-up, including those related to surveillance imaging, follow-up consultations, anti-hormonal treatment, treatment-induced menopausal symptoms, and lifestyle changes. Moreover, we identified decisions that were made during treatment, but that required additional decisions during follow-up, such as delayed breast reconstruction, hereditary testing, and pregnancy. The literature revealed that there was a large variety in the degree of preference sensitiveness and patient involvement with each decision during follow-up. Decisions about delayed breast reconstruction, for instance, were among those shown to be highly preference sensitive and for which many indications for patient-involvement existed. Equally, however, decisions were identified for which patients exhibited preferences, but for which they were not necessarily involved. Notably, this included decisions about the form, frequency, and length of surveillance imaging and follow-up consultations. Some decisions were not currently regarded as preference sensitive with a low recognition of the need for patient involvement, such as decisions about anti-hormonal therapy and the management of treatment-induced menopausal symptoms.

Notably, the data indicated that the patient's role and involvement should be improved for several decisions. First, regarding the form, frequency, and length of surveillance imaging, patients desired more frequent $[25,26]$ and intensive [26] surveillance; continuity of care and more frequent or longer appointments were preferences expressed in other studies already $[15,16]$. Despite these strong preferences, patients were rarely involved in making decisions, with physicians typically setting the imaging type and frequency [26]. However, this is probably a legitimate approach because guidelines provide clear, evidence-based recommendations about surveillance schemes and imaging modalities [4-7]. We suspect that the identified preferences were primarily based on the patient's need for reassurance $[25,26,58]$, and that they may be unaware that more intensive surveillance has no evidence base [59], or that increased exposure might even be harmful [60, 61]. Efforts should be made to improve patient understanding of the goals of surveillance [62], specifically at the point of transition from treatment to follow-up [26, 63]. Furthermore, the frequency and length of surveillance could be determined by recurrence risk stratification [61], based on data from nomograms or risk-calculators. Although Rabin et al. [64] reviewed 22 cancer prognostic tools, of which 8 focussed on breast cancer, patient-involvement with these tools was not discussed. The authors found only limited evidence reporting actual use of these in practice.

Issues also existed for follow-up consultations aimed at the physical and psychosocial effects of treatment. The available research indicated that patients preferred more frequent consultations than was recommended, that these should be led by specialised oncology-providers [25], and that these should be provided over a longer period of time [28]. As literature described unmet needs in information provision about follow-up, health promotion, late and long term-effects, or emotional and social needs [63,65-68], these preferences may be the result of these unmet needs. Moreover, $24 \%$ of patients sought care from multiple other providers [28], suggesting that referral 
for personalised care may sometimes be more appropriate than providing general oncology-led follow-up. We expect that using patient-reported outcome measures (PROMs) would help to identify patients' needs regarding specific forms of care [69]. PROMs can include symptom-specific scales about, for instance, physical impairments, sexuality problems, psychosocial problems, and body image [70, 71]. Patients and physicians would be able to discuss the results and subsequently ensure appropriate referrals to physiotherapists, sexologists, gynaecologists, medical social workers, psychologists, or plastic surgeons, as necessary.

Decisions about anti-hormonal treatment had little recognition as preference sensitive decisions among physicians, which is somewhat consistent with the 2015 European Society for Medical Oncology guideline. Although this guideline states that follow-up care should seek to motivate patients to continue anti-hormonal treatment [6], we should remember that patients must suffer many side-effects over a long period of time [29-32,34], and that this often occurs without proper counselling [29, 31, 32]. This leaves patients struggling to cope with difficult symptoms with minimal support [31]. Given that therapy-adherence depends on perseverance despite side-effects $[30,31]$, the needs and preferences of patients require more personalised attention in the longterm. This may be challenging, particularly for patients confronted with menopausal symptoms, for whom safe and effective evidence-based options for symptom relief are scarce [36, 37]. Finally, treatment-affected fertility in young premenopausal women may conflict with the desire to build a family, producing negative long term psychosocial effects $[29,54,55]$. These issues necessitate explicit information provision, counselling, and ongoing support to ensure treatment compliance and management of side effects $[67,72,73]$.

\section{Strengths and limitations}

Several limitations should be kept in mind when interpreting the results of this study. In the interview and focus-group studies, the samples included in these studies were small, which may limit the generalizability of the data. However, all the included studies were rated as valuable in the quality assessment.

We considered that the effectiveness of patient involvement or SDM is a separate research topic. Shay and Lafaya concluded that evidence about the association between empirical measures of SDM and patient behavioural and health outcomes is lacking. Given that SDM is not associated with improved outcomes, it should not be considered a goal in itself. However, because outcomes do tend to improve with personalised care, SDM may moderate some other factor [74].

\section{Practice implications and recommendations}

Currently, there is an international trend towards increased SDM in the diagnosis and treatment of all disease, based on the value-based healthcare initiative [17]. Further personalisation of followup care may lead to care that is not only of greater value for the individual patient, but also to care that is more appropriate from a financial perspective, potentially leading to more responsible use of available health care services as well. The process used when deciding on breast reconstruction may be considered an example of best-practise for other decisions about follow-up. Eight studies recommended improvement in information provision [38, 40,42, 45, 47, 48, 50, 52], and four 
reported on decision aids to address these information gaps [38, 43, 49, 50]. Although patientinvolvement seemed to be more straightforward when making elective decisions about breast reconstruction, true involvement in the decision-making process requires that patients be given the best available evidence, including details of the risks and benefits [18]. When the evidence for a certain decision is low, such as when making decisions about relieving menopausal symptoms, this uncertainty should be outlined by physicians [75].

\section{Conclusion}

We identified a variety of decisions that can be made about the content or form of follow-up care for patients with breast cancer. We grouped these into four categories: surveillance for recurrent or secondary breast cancer, consultations for physical and psychosocial (late) effects, recurrence risk reduction by anti-hormonal treatment, and improving quality of life. More attention should be given to the patient's role and the involvement in decisions where their input is both relevant and possible. Further personalisation of follow-up care may lead to care of greater relevance and value to individual patients. 


\section{References}

1. Bray F, Ferlay J, Soerjomataram I, Siegel RL, Torre LA, Jemal A. Global Cancer Statistics 2018: GLOBOCAN Estimates of Incidence and Mortality Worldwide for 36 Cancers in 185 Countries. CA: A Cancer Journal for Clinicians. 2018;0:1-31.

2. Janssen-Heijnen MLG, van Steenbergen LN, Voogd AC, Tjan-Heijnen VCG, Nijhuis PH, Poortmans PM, et al. Small but significant excess mortality compared with the general population for long-term survivors of breast cancer in the Netherlands. Annals of Oncology. 2014;25:64-8.

3. Howlader N, Noone AM, Krapcho M, Miller D, Bishop K, Kosary CL, et al. SEER Cancer Statistics Review, 1975-2014 Bethesda, MD: National Cancer Institute; 2017 [updated Based on November 2016 SEER data submission, posted to the SEER web site April 2017. Available from: https://seer.cancer.gov/csr/1975_2014/.

4. Netherlands Comprehensive Cancer Organisation (IKNL). National guideline on Breast Cancer. Netherlands Comprehensive Cancer Organisation (IKNL),,; 2012

5. Runowicz CD, Leach CR, Henry NL, Henry KS, Mackey HT, Cowens-Alvarado RL, et al. American Cancer Society/American Society of Clinical Oncology Breast Cancer Survivorship Care Guideline. Journal of Clinical Oncology. 2016;34(6):611-35.

6. Senkus E, Kyriakides S, Ohno S, Penault-Llorca F, Poortmans P, Rutgers E, et al. Primary breast cancer: ESMO Clinical Practice Guidelines for diagnosis, treatment and follow-up. Annals of Oncology. 2015;26:8-30.

7. Grunfeld E, Dhesy-Thind S, Levine M. Clinical practice guidelines for the care and treatment of breast cancer: follow-up after treatment for breast cancer (summary of the 2005 update). Canadian Medical Association Journal. 2005;172(10).

8. DH Macmillan Cancer Support, NHS Improvement. National Cancer Survivorship Initiative: Vision. 2010.

9. Donnelly P, Hiller L, Bathers S, Bowden S, Coleman R. Questioning specialists' attitudes to breast cancer follow-up in primary care. Annals of Oncology. 2007;18:1467-76.

10. Montgomery DA, Krupa K, Cooke TG. Alternative methods of follow up in breast cancer: a systematic review of the literature. British Journal of Cancer. 2007;96:1625-32.

11. Zorginstituut Nederland. Verbetersignalement Zinnige nacontrole bij vrouwen behandeld voor borstkanker. Diemen: Zorginstituut Nederland; 2016.

12. Witteveen A, Vliegen IMH, Sonke GS, Klaase JM, IJzerman MJ, Siesling S. Personalisation of breast cancer follow-up: a time-dependent prognostic nomogram for the estimation of annual risk of locoregional recurrence in early breast cancer patients. Breast Cancer Research and Treatment. 2015;152(3):627-36.

13. IJzerman M, Hans E, Siesling S, Klaase J, editors. Optimization of follow-up scenarios following breast cancer: poster. 33rd Annual Meeting of the Society for Medical Decision Making, SMDM 2011; 2011 October 22-26; Chicago, IL, USA.

14. Ewertz $M$, Jensen $A B$. Late effects of breast cancer treatment and potentials for rehabilitation. Acta Oncologica. 2011;50:187-93.

15. Kimman ML DB, Boersma LJ, Lambin P, Dirksen CD. Follow-up after treatment for breast cancer: One strategy fits all? An investigation of patient preferences using a discrete choice experiment. Acta Oncol. 2010;49:32837.

16. Murchie P, Norwood PF, Pietrucin-Materek M, Porteous T, Hannaford PC, Ryan M. Determining cancer survivors' preferences to inform new models of follow-up care. British Journal of Cancer 2016;115(14951503).

17. Porter ME, Teisberg EO. How Physicians Can Change the Future of Health Care. JAMA. 2007;297(10):110311.

18. Légaré F, Ratté S, Gravel K, Graham ID. Barriers and facilitators to implementing shared decision-making in clinical practice: update of a systematic review of health professionals' perceptions. Patient Educ Couns. 2008;73(3):526-35.

19. van de Haterd J, Voogdt-Pruis H, Raats I, van den Brink R, van Veenendaal H. Preference-sensitive decisions of patients with metastatic breast cancer: The need for decision support. Patient Experience Journal. 2016;3(1).

20. University of York Centre for Reviews and Dissemination, editor. PROSPERO: international prospective register of systematic reviews: National Institute for Health Research,,. 
21. Clarivate Analytics. EndNote X7. Clarivate Analytics,,

22. Critical Appraisal Skills Programme (CASP). CASP Checklist. Oxford.

23. van der Weijden T, Pieterse AH, Koelewijn-van Loon MS, Knaapen L, Légaré F, Boivin A, et al. How can clinical practice guidelines be adapted to facilitate shared decision making? A qualitative key-informant study. BMJ Quality \& Safety. 2013;22:855-63.

24. Coulter A, Collins A. Making shared decision-making a reality: no decision about me, without me: The Kings Fund; 2011.

25. Klaassen LA, Dirksen CD, Boersma LJ, Hoving C. Developing an aftercare decision aid; assessing health professionals' and patients' preferences. Eur J Cancer Care (Engl). 2018;27(2).

26. Brandzel S, Rosenberg DE, Johnson D, Bush M, Kerlikowske K, Onega T, et al. Women's experiences and preferences regarding breast imaging after completing breast cancer treatment. Patient preference and adherence. 2017;11:199-204.

27. Rini C, O'Neill SC, Valdimarsdottir H, Goldsmith RE, Jandorf L, Brown K, et al. Cognitive and emotional factors predicting decisional conflict among high-risk breast cancer survivors who receive uninformative BRCA1/2 results. Health Psychology. 2009;28(5):569-78.

28. Hudson SV, Miller SM, Hemler J, McClinton A, Oeffinger KC, Tallia A, et al. Cancer survivors and the patientcentered medical home. Translational Behavioral Medicine. 2012;2(3):322-31.

29. Benedict C, Thom B, Teplinsky E, Carleton J, Kelvin JF. Family-building After Breast Cancer: Considering the Effect on Adherence to Adjuvant Endocrine Therapy. BMC bioinformatics. 2017;17(3):165-70.

30. Bluethmann SM, Murphy CC, Tiro JA, Mollica MA, Vernon SW, Bartholomew LK. Deconstructing Decisions to Initiate, Maintain, or Discontinue Adjuvant Endocrine Therapy in Breast Cancer Survivors: A Mixed-Methods Study. Oncol Nurs Forum. 2017;44(3):E101-e10.

31. Brauer ER, Ganz PA, Pieters HC. "Winging It": How Older Breast Cancer Survivors Persist With Aromatase Inhibitor Treatment. Journal of oncology practice. 2016;12(12):e991-e1000.

32. Cahir C, Dombrowski SU, Kelly CM, Kennedy MJ, Bennett K, Sharp L. Women's experiences of hormonal therapy for breast cancer: exploring influences on medication-taking behaviour. Supportive care in cancer : official journal of the Multinational Association of Supportive Care in Cancer. 2015;23(11):3115-30.

33. Engelhardt EG, Pieterse AH, van der Hout A, de Haes HJCJM, Kroep JR, Quarles van Ufford-Mannesse P, et al. Use of implicit persuasion in decision making about adjuvant cancer treatment: A potential barrier to shared decision making. European Journal of Cancer. 2016;66:55-66.

34. Hershman DL, Kushi LH, Hillyer GC, Coromilas E, Buono D, Lamerato L, et al. Psychosocial factors related to non-persistence with adjuvant endocrine therapy among women with breast cancer: the Breast Cancer Quality of Care Study (BQUAL). Breast Cancer Res Treat. 2016;157(1):133-43.

35. Neugut Al, Hillyer GC, Kushi LH, Lamerato L, Leoce N, Nathanson SD, et al. Non-initiation of adjuvant hormonal therapy in women with hormone receptor-positive breast cancer: The Breast Cancer Quality of Care Study (BQUAL). Breast Cancer Res Treat. 2012;134(1):419-28.

36. Balneaves LG, Panagiotoglou D, Brazier AS, Lambert LK, Porcino A, Forbes M, et al. Qualitative assessment of information and decision support needs for managing menopausal symptoms after breast cancer. Archives of gynecology and obstetrics. 2016;24(11):4567-75.

37. Sayakhot $P$, Vincent A, Teede $H$. Breast cancer and menopause: perceptions of diagnosis, menopausal therapies and health behaviors. Climacteric : the journal of the International Menopause Society. 2012;15(1):59-67.

38. Causarano N, Platt J, Baxter NN, Bagher S, Jones JM, Metcalfe KA, et al. Pre-consultation educational group intervention to improve shared decision-making for postmastectomy breast reconstruction: a pilot randomized controlled trial. Supportive care in cancer : official journal of the Multinational Association of Supportive Care in Cancer. 2015;23(5):1365-75.

39. Flitcroft K, Brennan M, Costa D, Wong A, Snook K, Spillane A. An evaluation of factors affecting preference for immediate, delayed or no breast reconstruction in women with high-risk breast cancer. Psycho-Oncology. 2016;25(12):1463-9.

40. Potter S, Mills N, Cawthorn S, Wilson S, Blazeby J. Exploring inequalities in access to care and the provision of choice to women seeking breast reconstruction surgery: a qualitative study. Br J Cancer. 2013;109(5):118191. 
41. Zielinski T, Lorenc-Podgorska K, Antoszewski B. Why women who have mastectomy decide not to have breast reconstruction? Polski przeglad chirurgiczny. 2015;86(10):451-5.

42. Alderman AK, Hawley ST, Morrow M, Salem B, Hamilton A, Graff JJ, et al. Receipt of delayed breast reconstruction after mastectomy: do women revisit the decision? Ann Surg Oncol. 2011;18(6):1748-56.

43. Sherman KA, Shaw LK, Winch CJ, Harcourt D, Boyages J, Cameron LD, et al. Reducing Decisional Conflict and Enhancing Satisfaction with Information among Women Considering Breast Reconstruction following Mastectomy: Results from the BRECONDA Randomized Controlled Trial. Plast Reconstr Surg. 2016;138(4):592e-602e.

44. Lee CN, Hultman CS, Sepucha K. Do patients and providers agree about the most important facts and goals for breast reconstruction decisions? Ann Plast Surg. 2010;64(5):563-6.

45. Ogrodnik A, MacLennan S, Weaver D, James T. Barriers to Completing Delayed Breast Reconstruction Following Mastectomy: a Critical Need for Patient and Clinician Education. Journal of cancer education : the official journal of the American Association for Cancer Education. 2016.

46. Fasse L, Flahault C, Vioulac C, Lamore K, Van Wersch A, Quintard B, et al. The decision-making process for breast reconstruction after cancer surgery: Representations of heterosexual couples in long-standing relationships. British journal of health psychology. 2017;22(2):254-69.

47. Fu R, Chang MM, Chen M, Rohde $\mathrm{CH}$. A Qualitative Study of Breast Reconstruction Decision-Making among Asian Immigrant Women Living in the United States. Plast Reconstr Surg. 2017;139(2):360e-8e.

48. Hamnett KE, Subramanian A. Breast reconstruction in older patients: A literature review of the decisionmaking process. J Plast Reconstr Aesthet Surg. 2016;69(10):1325-34.

49. Temple-Oberle C, Ayeni O, Webb C, Bettger-Hahn M, Ayeni O, Mychailyshyn N. Shared decision-making: applying a person-centered approach to tailored breast reconstruction information provides high satisfaction across a variety of breast reconstruction options. Journal of surgical oncology. 2014;110(7):796-800.

50. Heller L, Parker PA, Youssef A, Miller MJ. Interactive digital education aid in breast reconstruction. Plast Reconstr Surg. 2008;122(3):717-24.

51. Kadmon I, Noy S, Billig A, Tzur T. Decision-Making Styles and Levels of Involvement Concerning Breast Reconstructive Surgery: An Israeli Study. Oncol Nurs Forum. 2016;43(1):E1-7.

52. Morrow M, Li Y, Alderman AK, Jagsi R, Hamilton AS, Graff JJ, et al. Access to breast reconstruction after mastectomy and patient perspectives on reconstruction decision making. JAMA surgery. 2014;149(10):101521.

53. Corney RH, Swinglehurst AJ. Young childless women with breast cancer in the UK: A qualitative study of their fertility-related experiences, options, and the information given by health professionals. Psycho-Oncology. 2014;23(1):20-6.

54. Gorman JR, Usita PM, Madlensky L, Pierce JP. Young breast cancer survivors: Their perspectives on treatment decisions and fertility concerns. Cancer Nursing. 2011;34(1):32-40.

55. Hsieh PL, Huang SM. Risk-benefit perception of pregnancy among breast cancer survivors. European Journal of Cancer Care. 2017;00:e12696.

56. Carter CL, Onicescu G, Cartmell KB, Sterba KR, Tomsic J, Fox T, et al. Factors associated with cancer survivors' selection between two group physical activity programs. Journal of Cancer Survivorship. 2010;4(4):388-98.

57. Shtaynberger J, Krebs P. Associations between decisional balance and health behaviors among adult cancer survivors. Journal of Cancer Education. 2016;31(4):749-54.

58. Allen A. The meaning of the breast cancer follow-up experience for the women who attend. European Journal of Oncology Nursing. 2002;6(3):155-61.

59. Rosselli Del Turco M, Palli D, Cariddi A, Ciatto S, Pacini P, Distante V. Intensive diagnostic follow-up after treatment of primary breast cancer. A randomized trial. National Research Council Project on Breast Cancer follow-up. JAMA. 1994;271(20):1593-7.

60. Meyer C, Millan P, Gonzalez V, Spera G, Machado A, Mackey JR, et al. Intensive Imaging Surveillance of Survivors of Breast Cancer May Increase Risk of Radiation-induced Malignancy. Clinical breast cancer. 2019.

61. Grunfeld EA. Optimizing follow-up after breast cancer treatment. Current Opinion in Obstetrics and Gynecology. 2009;21:92-6. 
62. Kwast ABG, Drossaert CHC, Siesling S. Breast cancer follow-up: from the perspective of health professionals and patients. European Journal of Cancer Care. 2013;22:754-64.

63. Schmidt A, Ernstmann N, Wesselmann S, Pfaff H, Wirtz M, Kowalski C. After initial treatment for primary breast cancer: information needs, health literacy, and the role of health care workers. Supportive care in cancer : official journal of the Multinational Association of Supportive Care in Cancer. 2016;24:563-71.

64. Rabin BA, Gaglio B, Sanders T, Nekhlyudov L, Dearing JW, Bull S, et al. Predicting cancer prognosis using interactive online tools: a systematic review and implications for cancer care providers. Cancer epidemiology, biomarkers \& prevention : a publication of the American Association for Cancer Research, cosponsored by the American Society of Preventive Oncology. 2013;22(10):1645-56.

65. Chawla N, Blanch-Hartigan D, Virgo KS, Ekwueme DU, Han X, Forsythe L, et al. Quality of Patient-Provider Communication Among Cancer Survivors: Findings From a Nationally Representative Sample. Journal of Oncology Practise. 2016;12(12).

66. Kent EE, Arora NK, Rowland JH, Bellizzi KM, LLP F, Hamilton AS, et al. Health information needs and healthrelated quality of life in a diverse population of long-term cancer survivors. Patient Educ Couns. 2012;89:34552.

67. Meade E, Mcllfatrick S, Groarke AM, Butler E, Dowling M. Survivorship care for postmenopausal breast cancer patients in Ireland: What do women want? European Journal of Oncology Nursing. 2017;28:69-76.

68. Binkley JM, Harris SR, Levangie PK, Pearl M, Guglielmino J, Kraus V, et al. Patient Perspectives on Breast Cancer Treatment Side Effects and the Prospective Surveillance Model for Physical Rehabilitation for Women With Breast Cancer. Cancer. 2012;118:2207-16.

69. Black N. Patient reported outcome measures could help transform healthcare. British Medical Journal. 2013;346:f167.

70. Cano SJ, Klassen AF, Scott AM, Pusic AL. A Closer Look at the BREAST-Q. Clin Plastic Surg. 2013;40:287-96.

71. Wintner LM, Sztankay M, Aaronson N, Bottomley A, Giesinger JM, Groenvold M, et al. The use of EORTC measures in daily clinical practiced $A$ synopsis of a newly developed manual. European Journal of Cancer. 2016;68:73-81.

72. Cardoso F, Loibl S, Pagani O, Graziottin A, Panizza P, Martincich L, et al. The European Society of Breast Cancer Specialists recommendations for the management of young women with breast cancer. European journal of cancer (Oxford, England : 1990). 2012;48:3355- 77.

73. Howard-Anderson J, Ganz PA, Bower JE, Stanton AL. Quality of Life, Fertility Concerns, and Behavioral Health Outcomes in Younger Breast Cancer Survivors: A Systematic Review. J Natl Cancer Inst. 2012;104:386-405.

74. Shay LA, Lafata JE. Where is the evidence? A systematic review of shared decision making and patient outcomes. Med Decis Making. 2015;35(1):114-31.

75. Politi MC, Lewis CL, Frosch DL. Supporting Shared Decisions When Clinical Evidence Is Low. Medical Care Research and Review. 2013;70(1):113S-28S. 


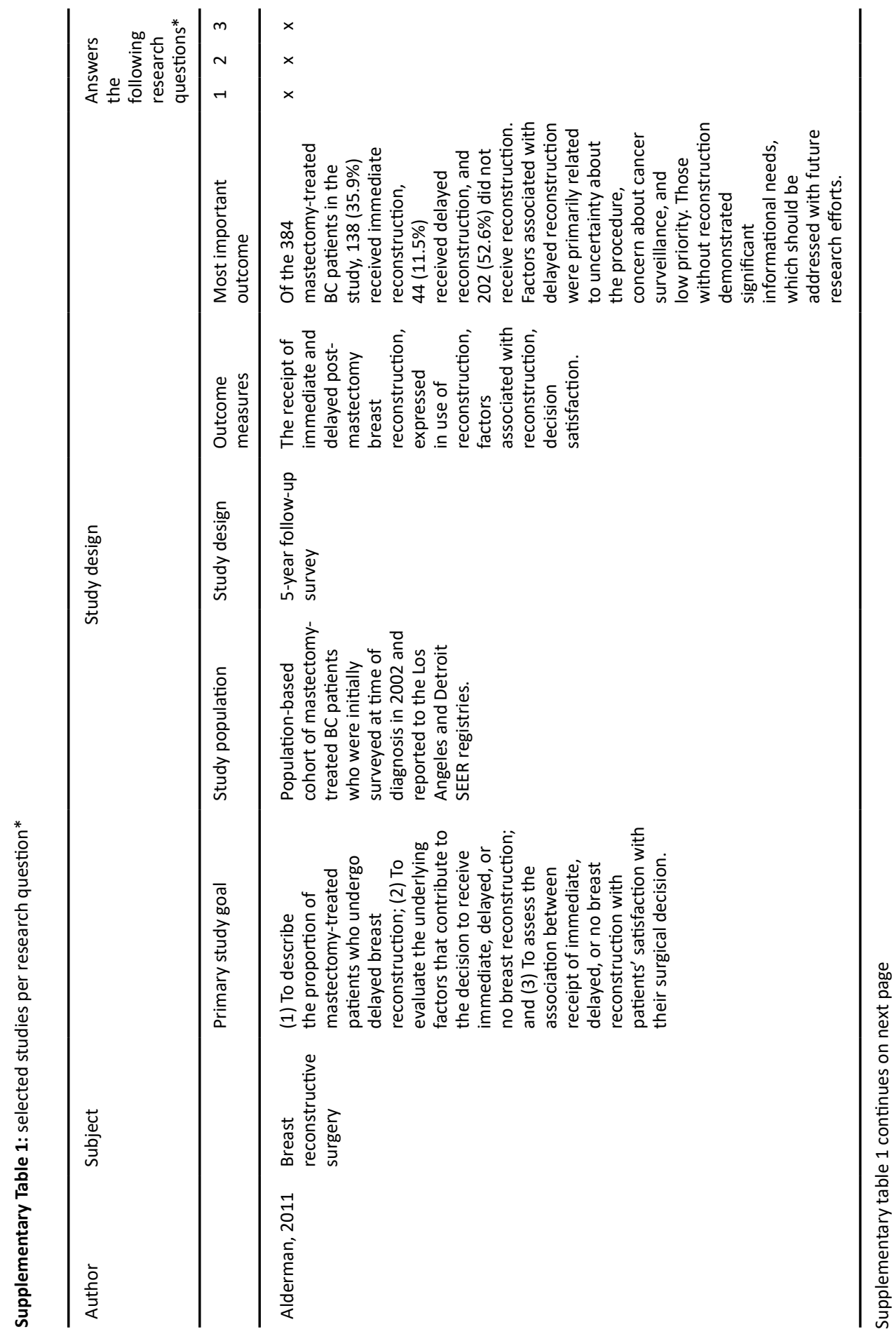




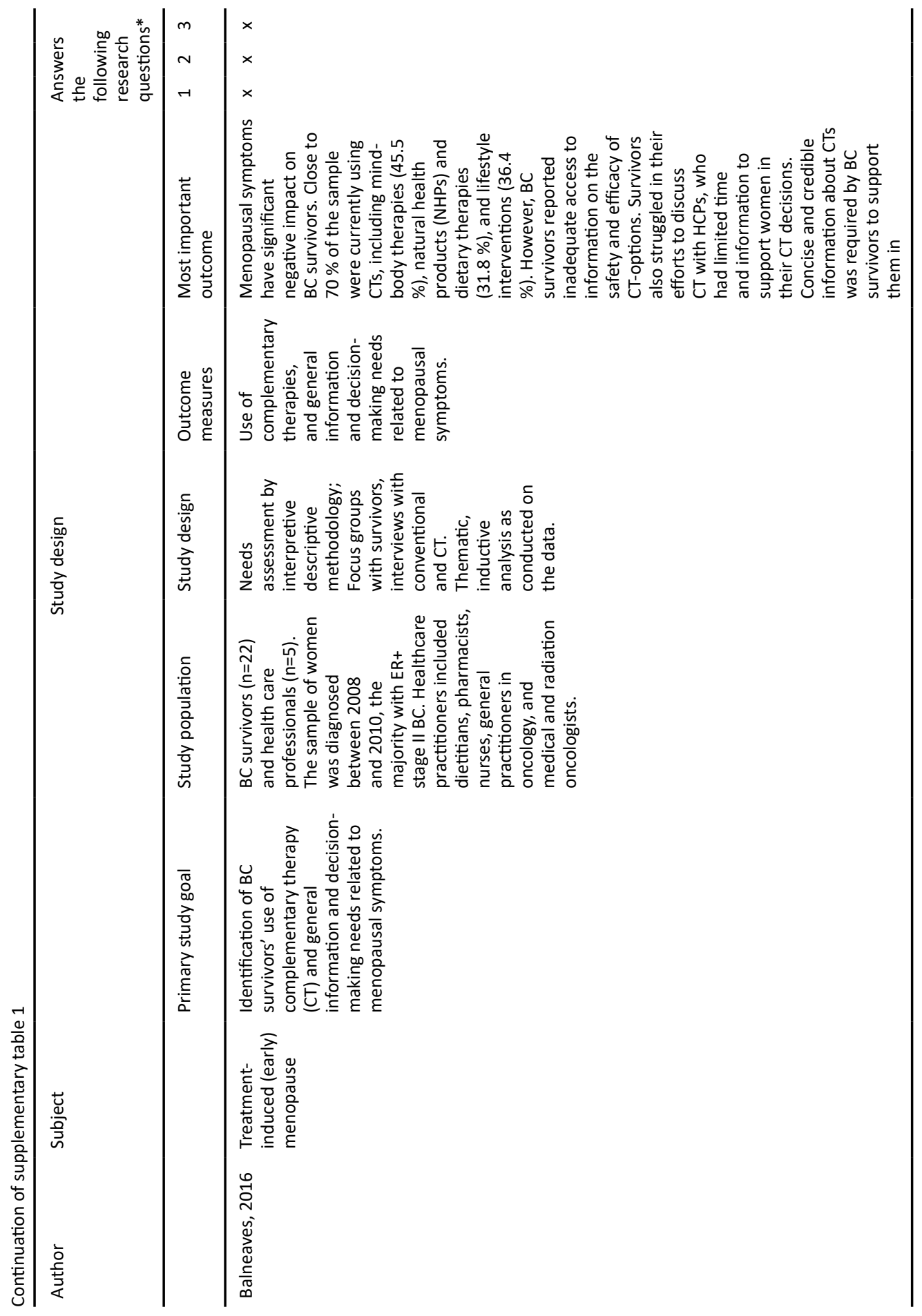




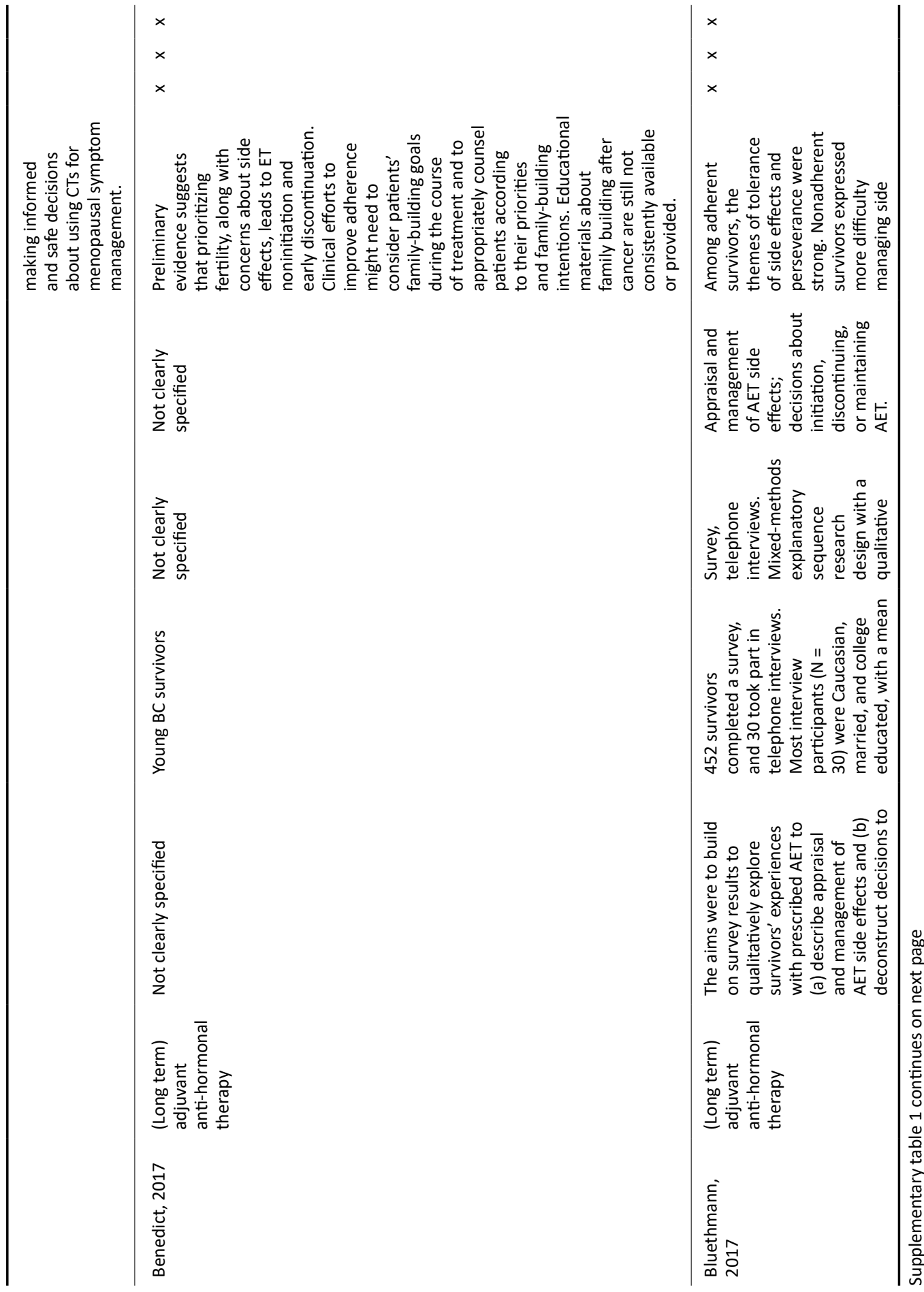




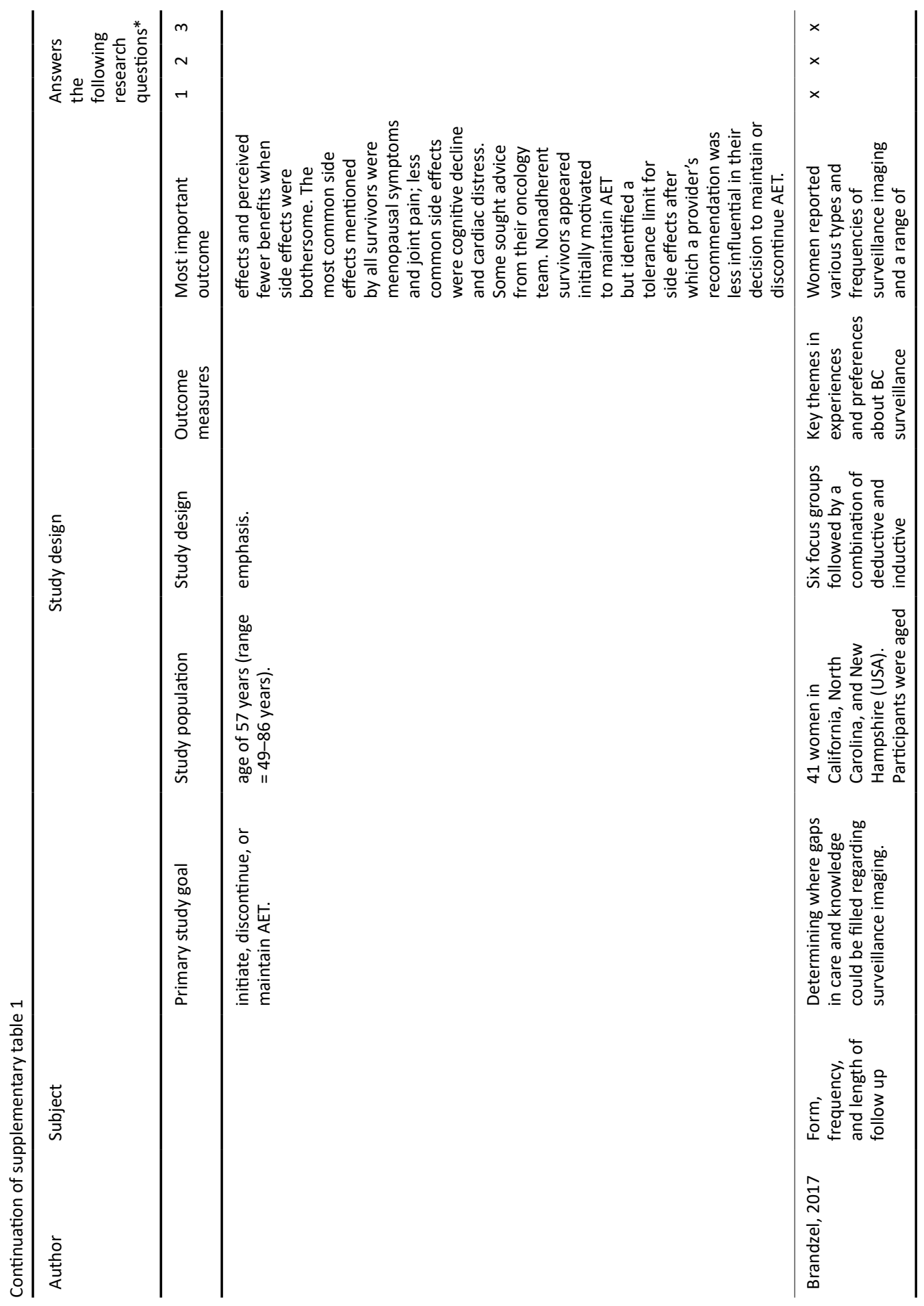




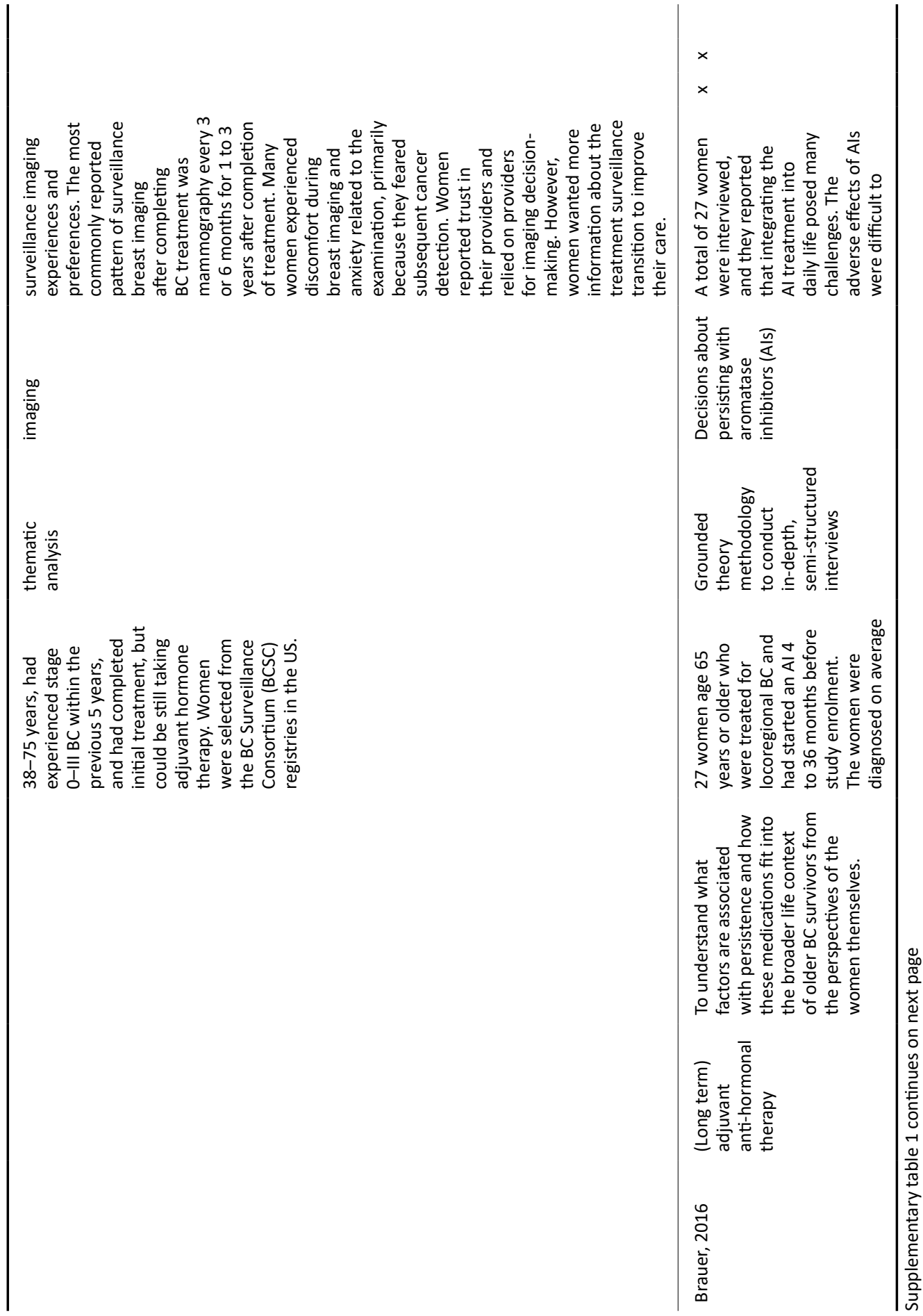




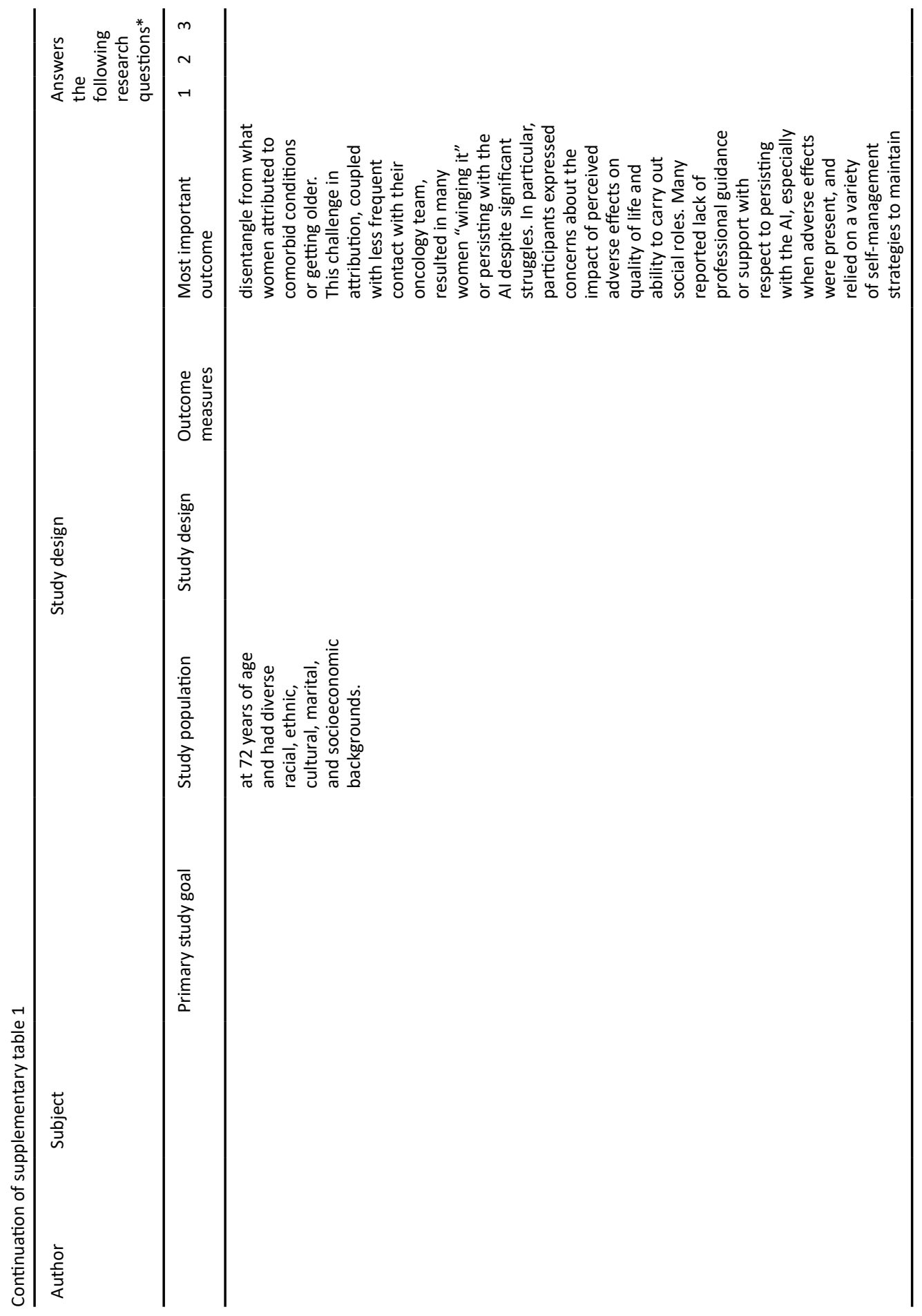




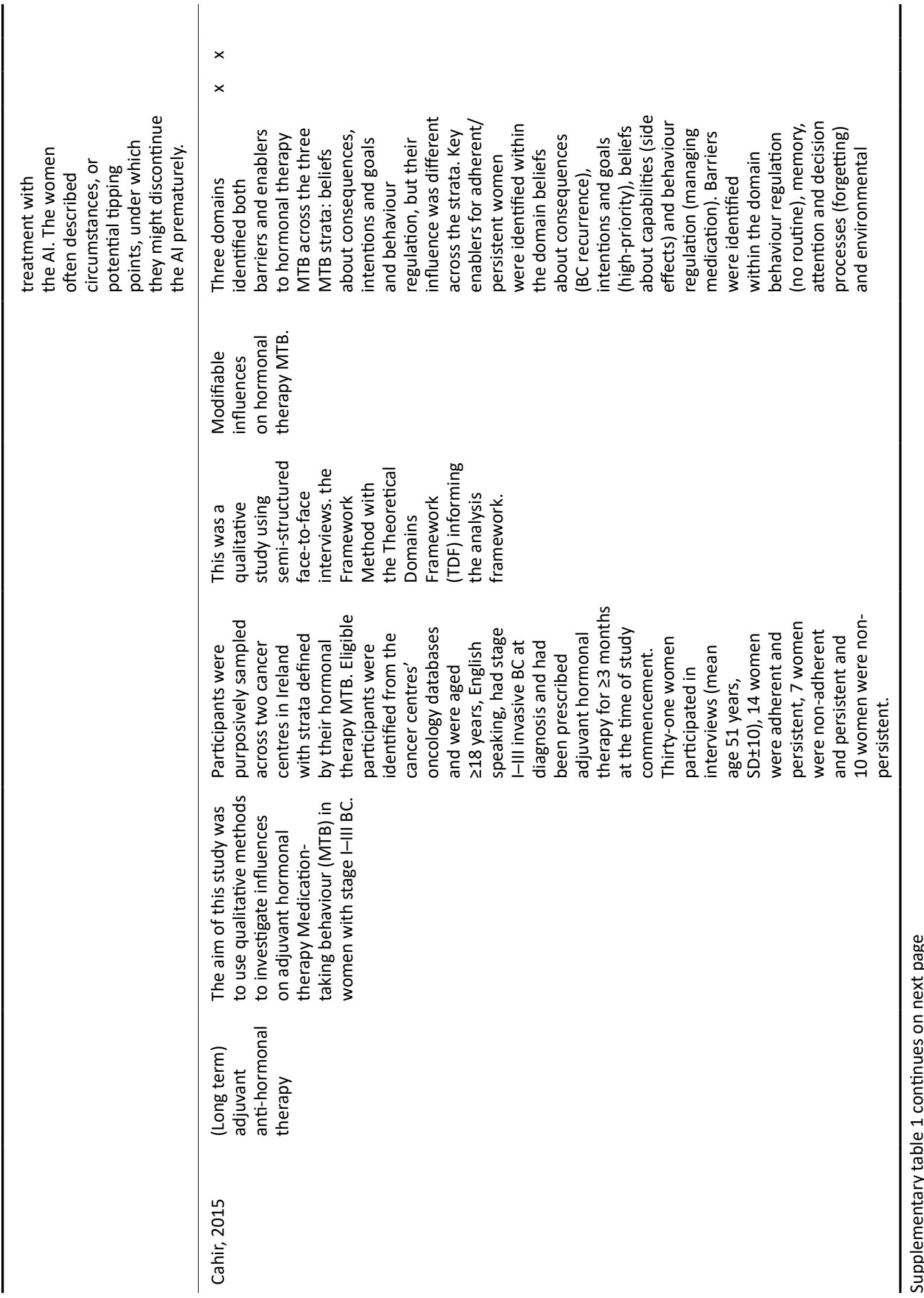




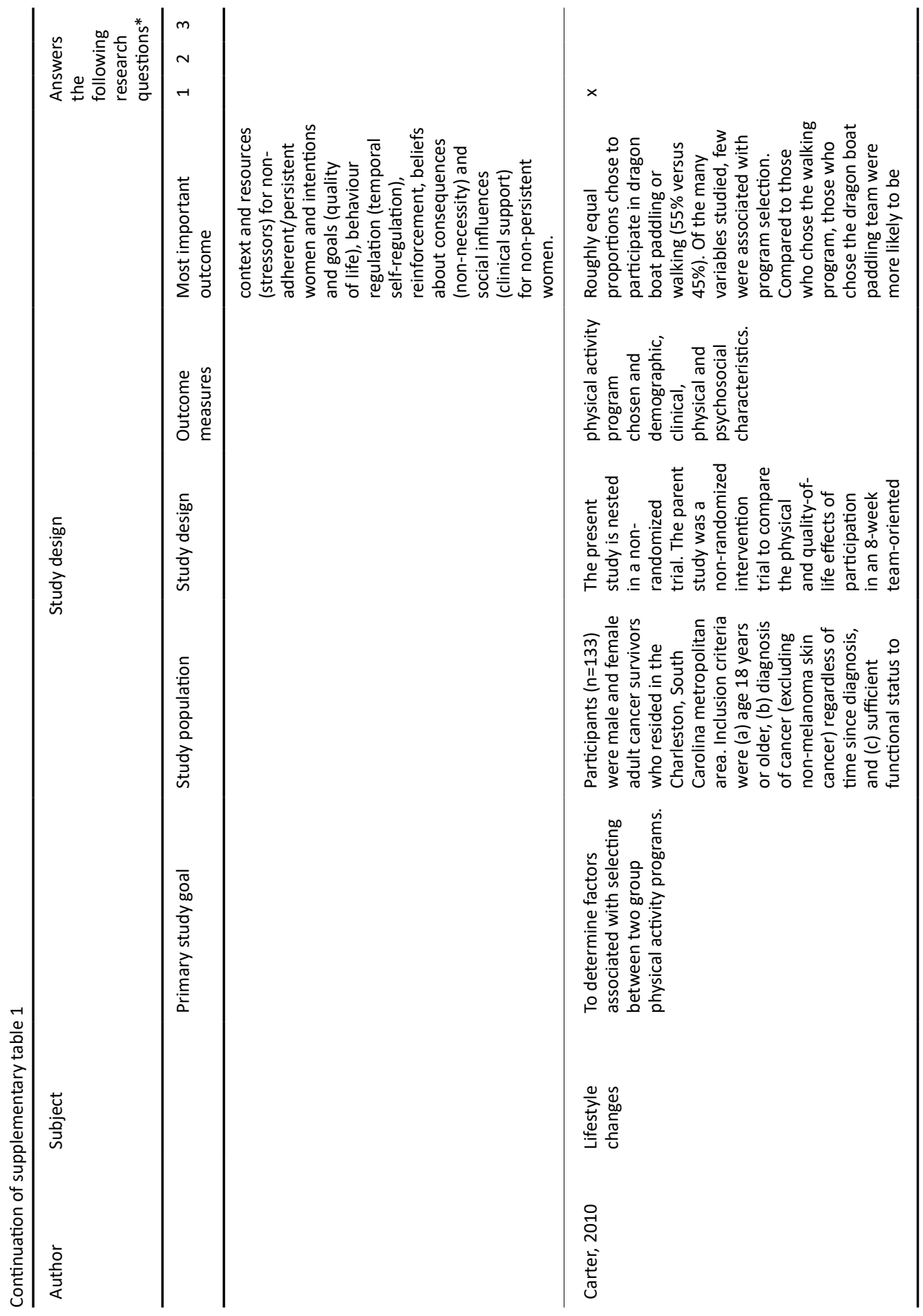




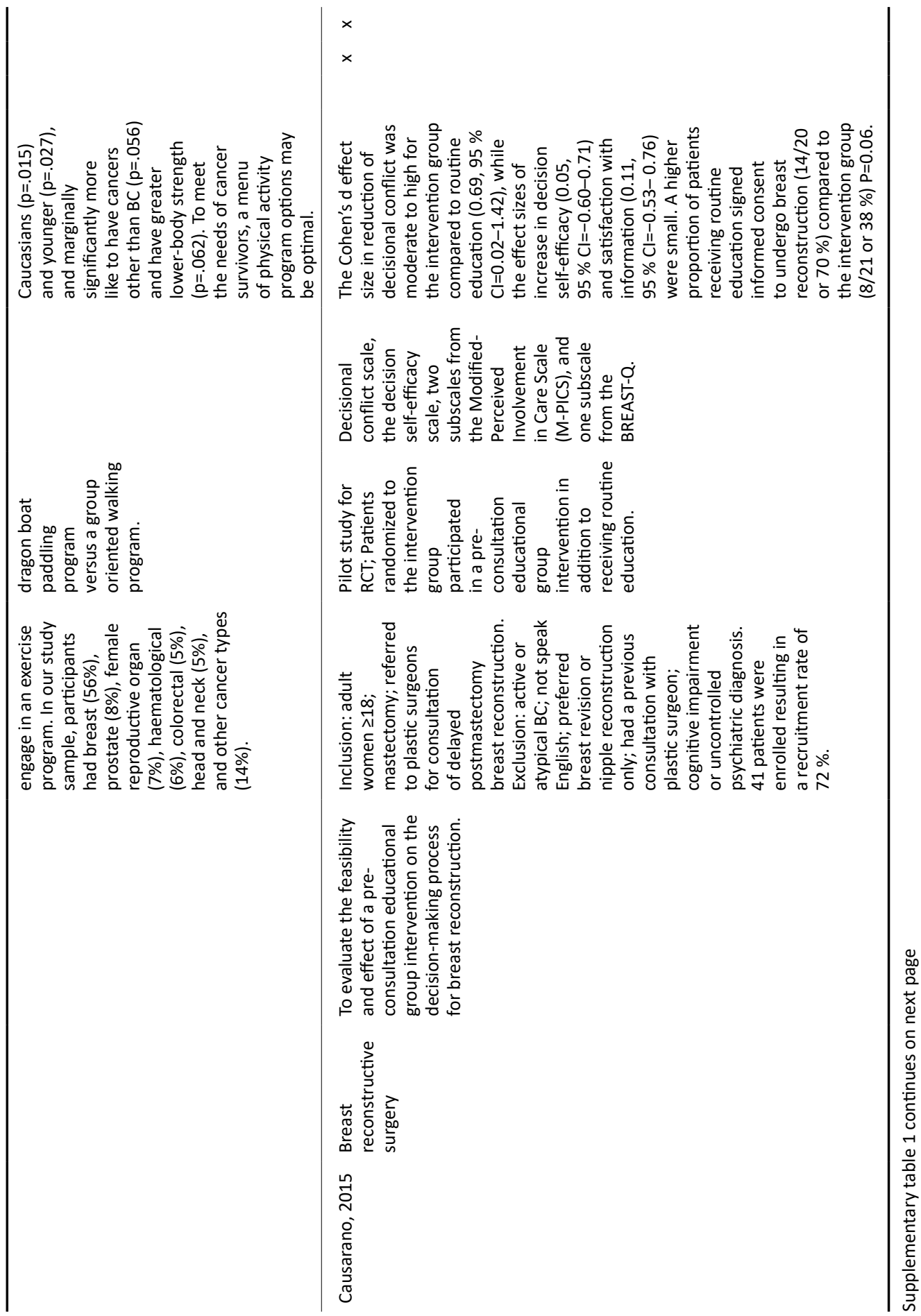




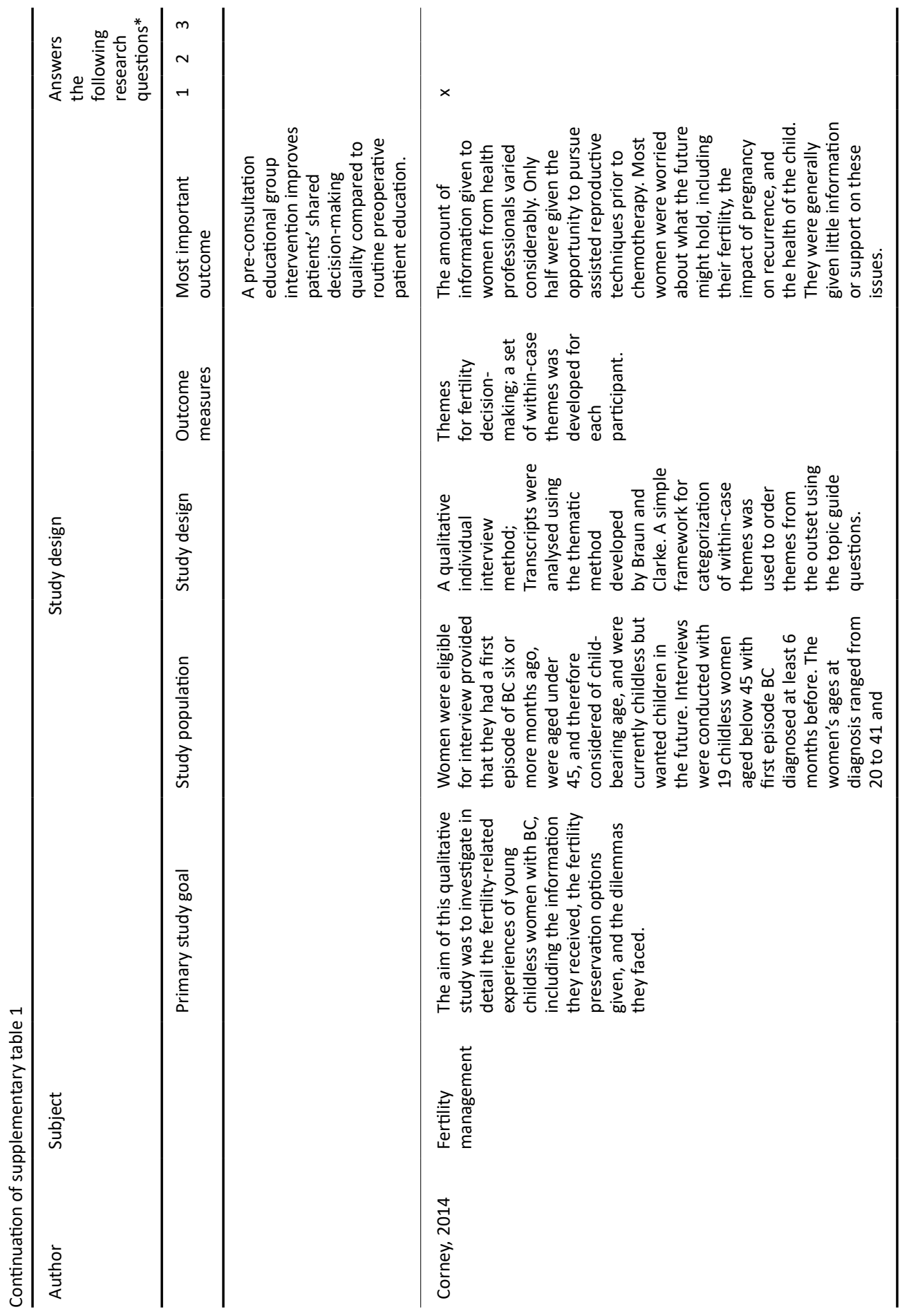




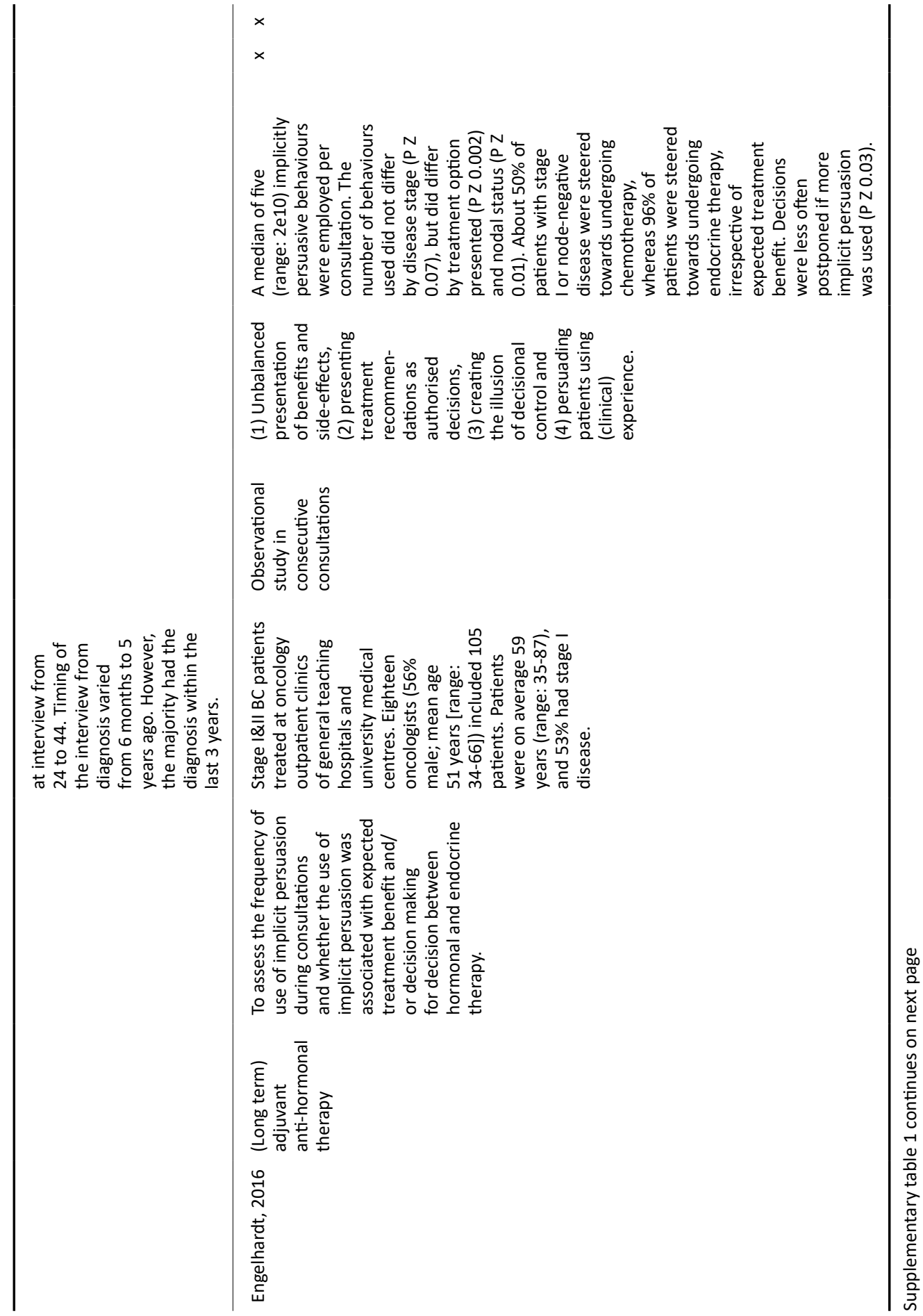




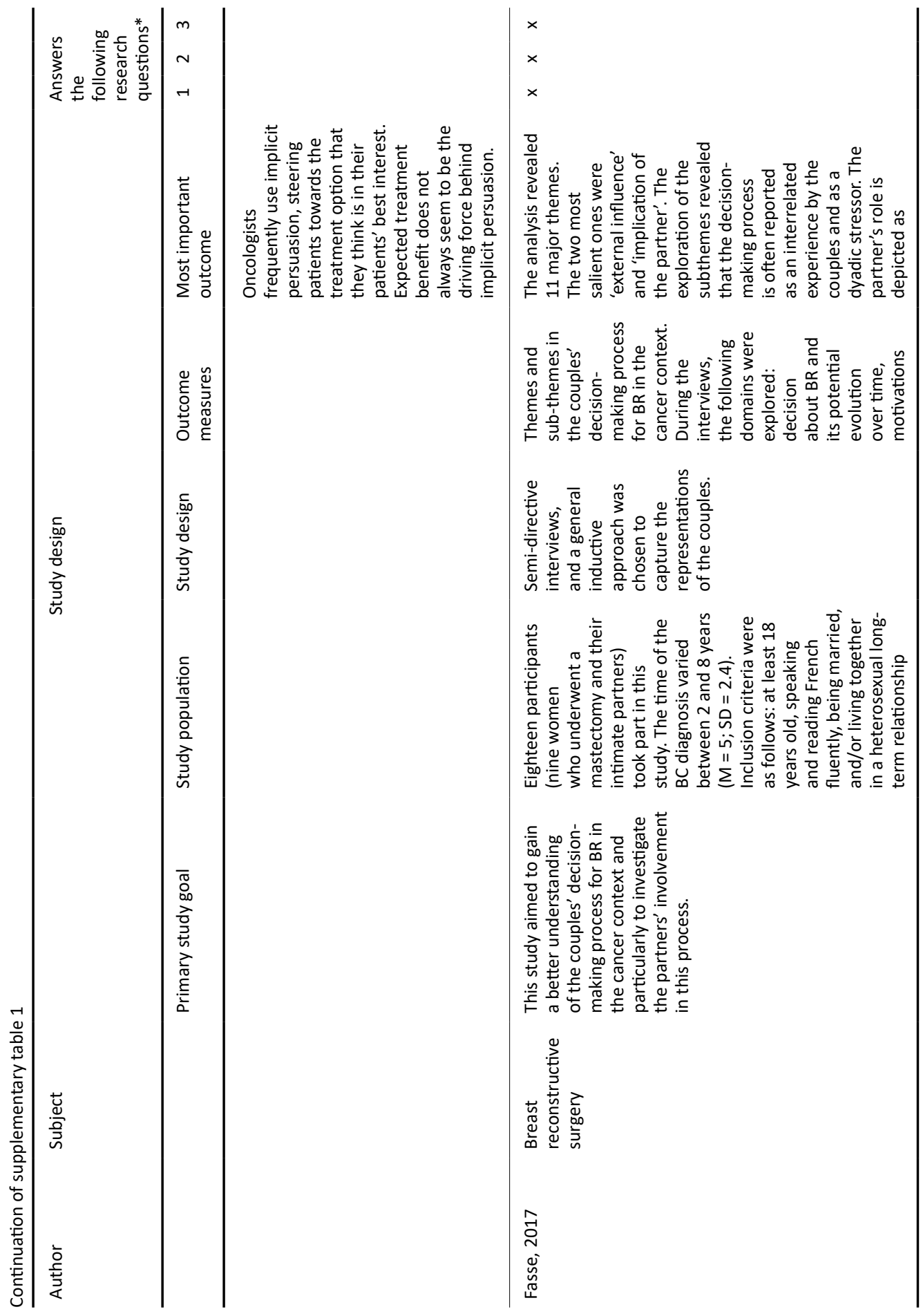




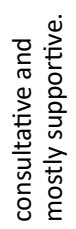

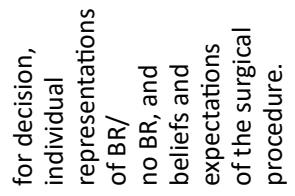

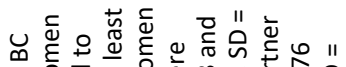

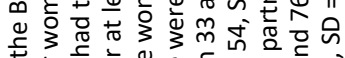

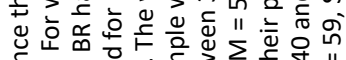

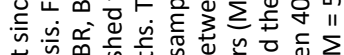

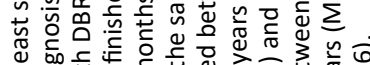

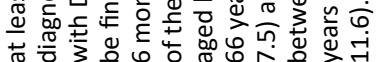

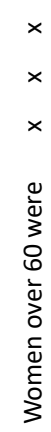

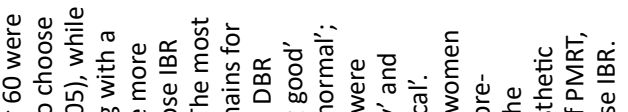

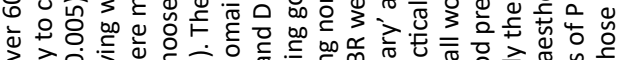

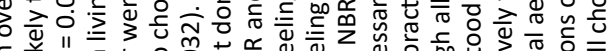

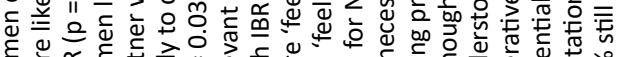

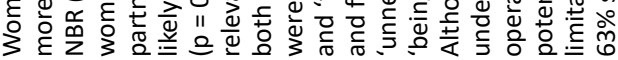

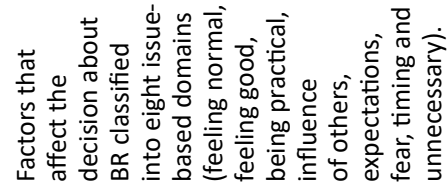

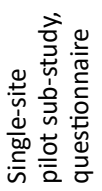

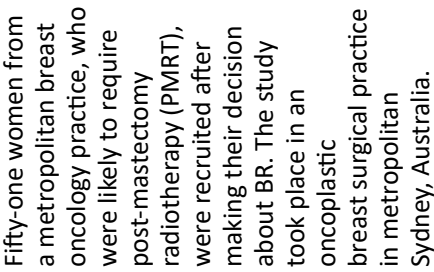

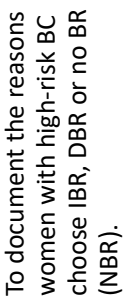

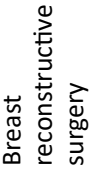

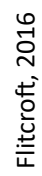

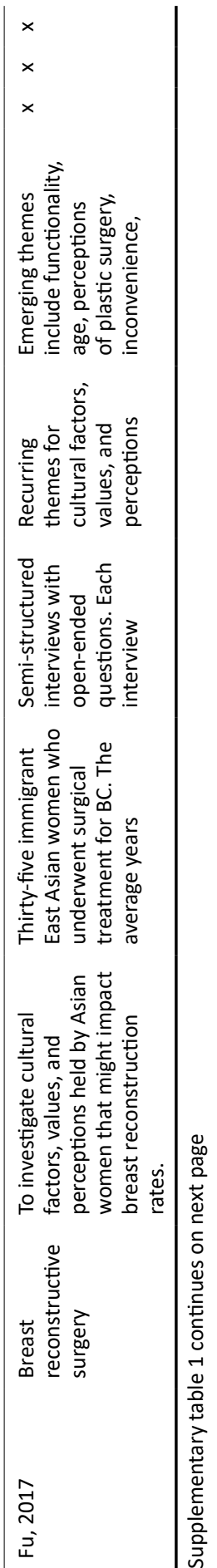




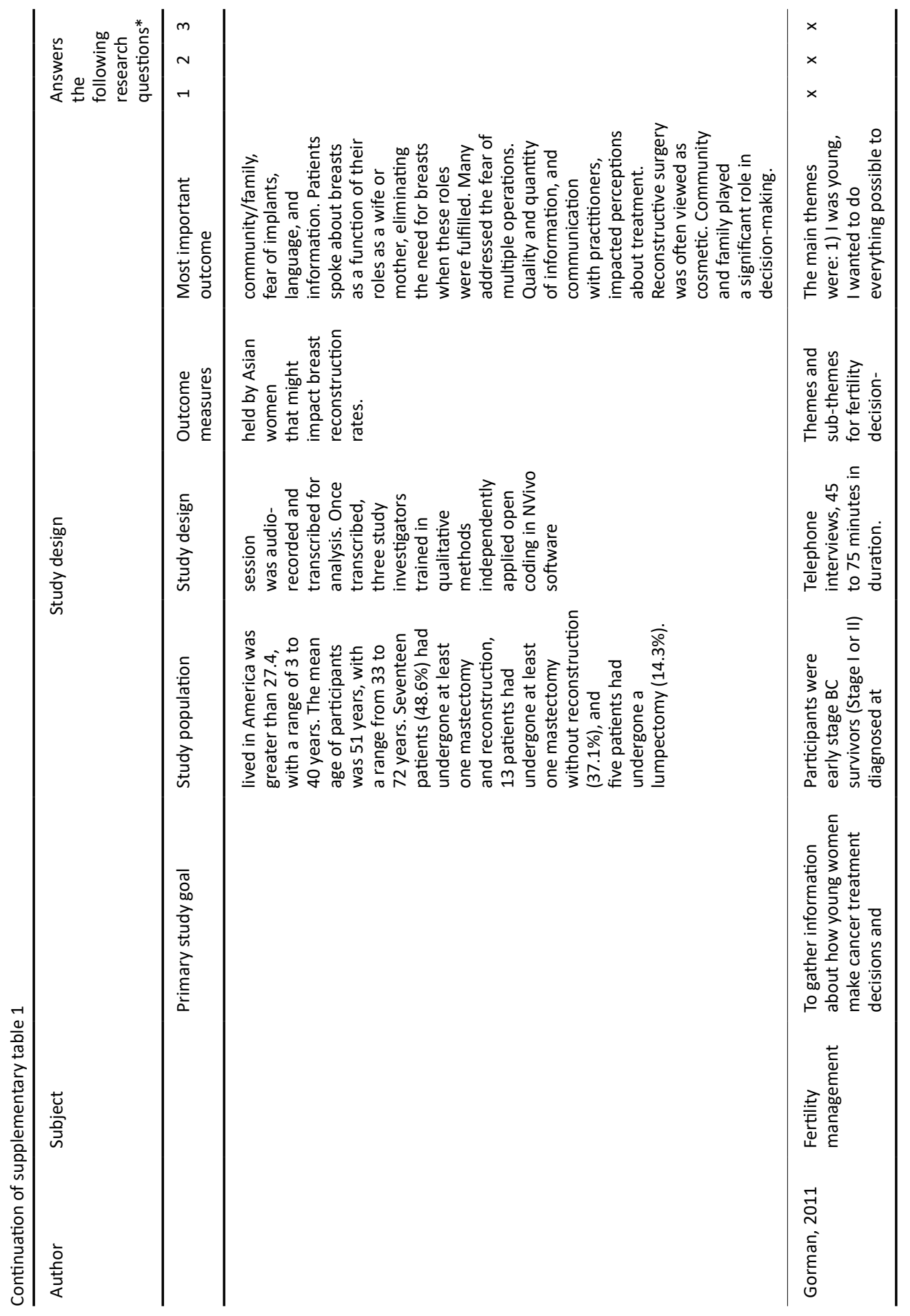




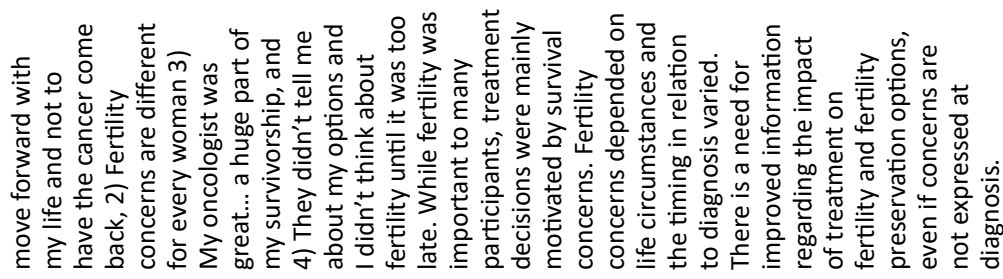

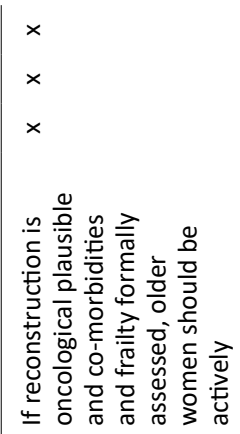

离

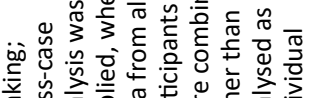

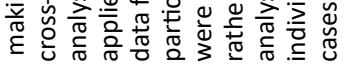

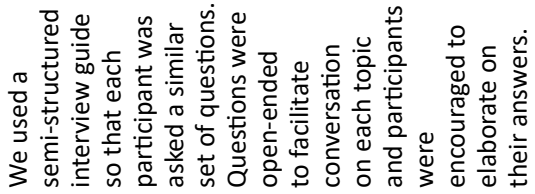

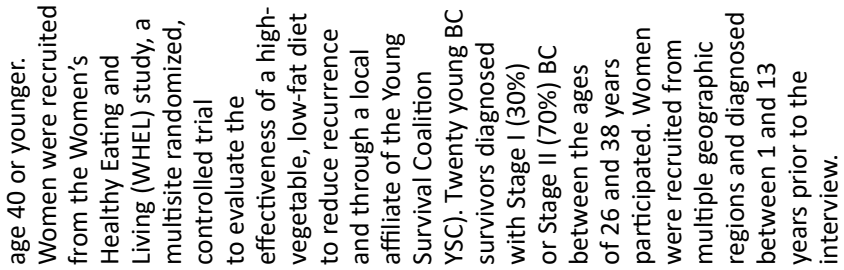

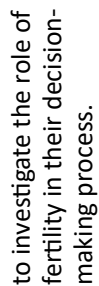

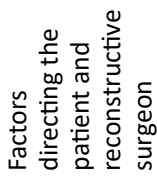

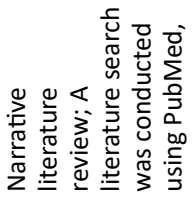

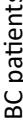

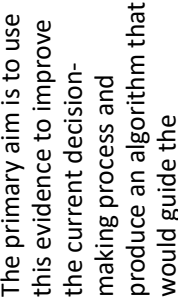

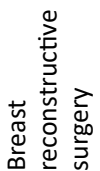

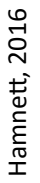

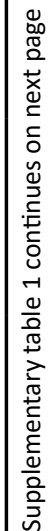




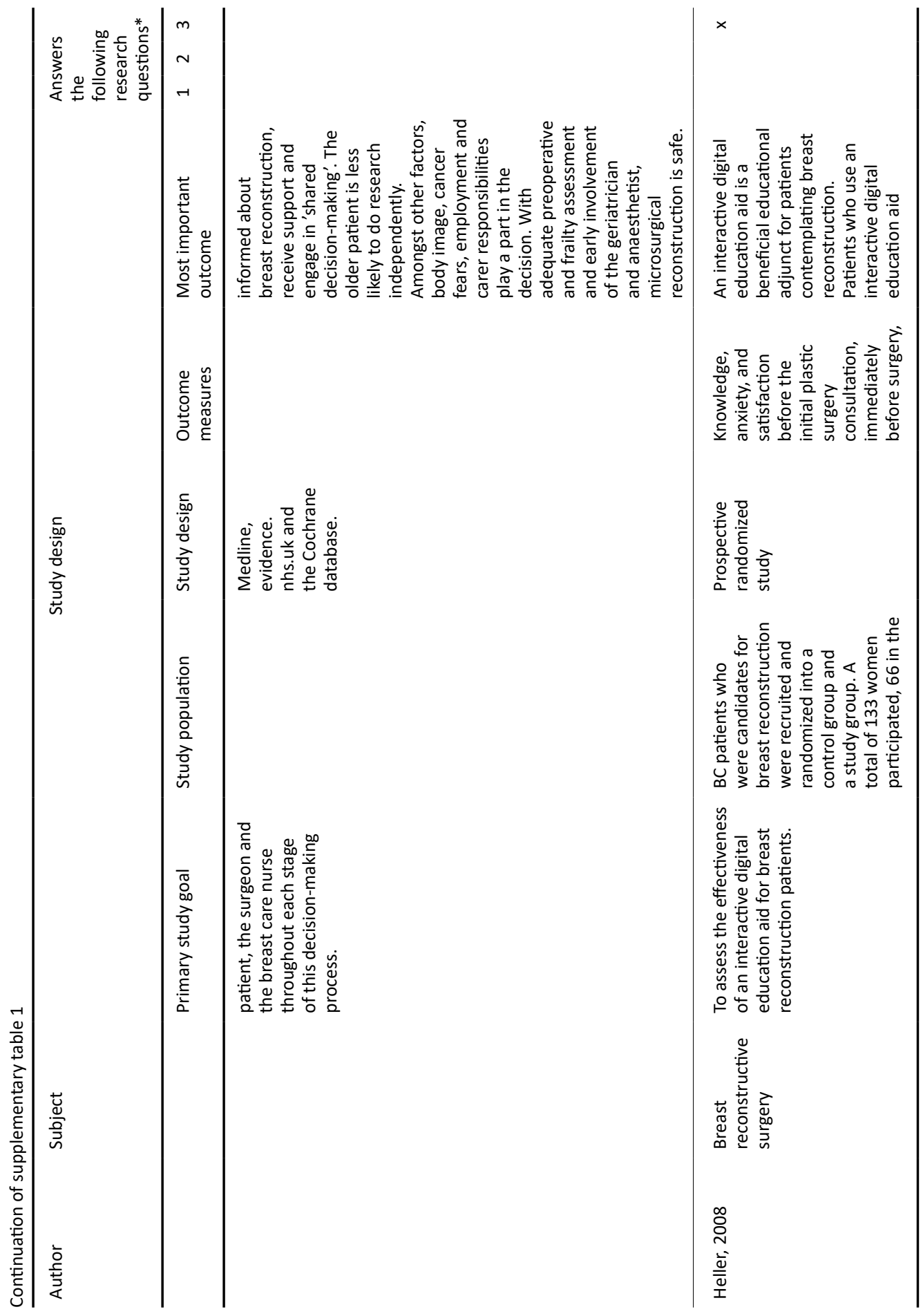




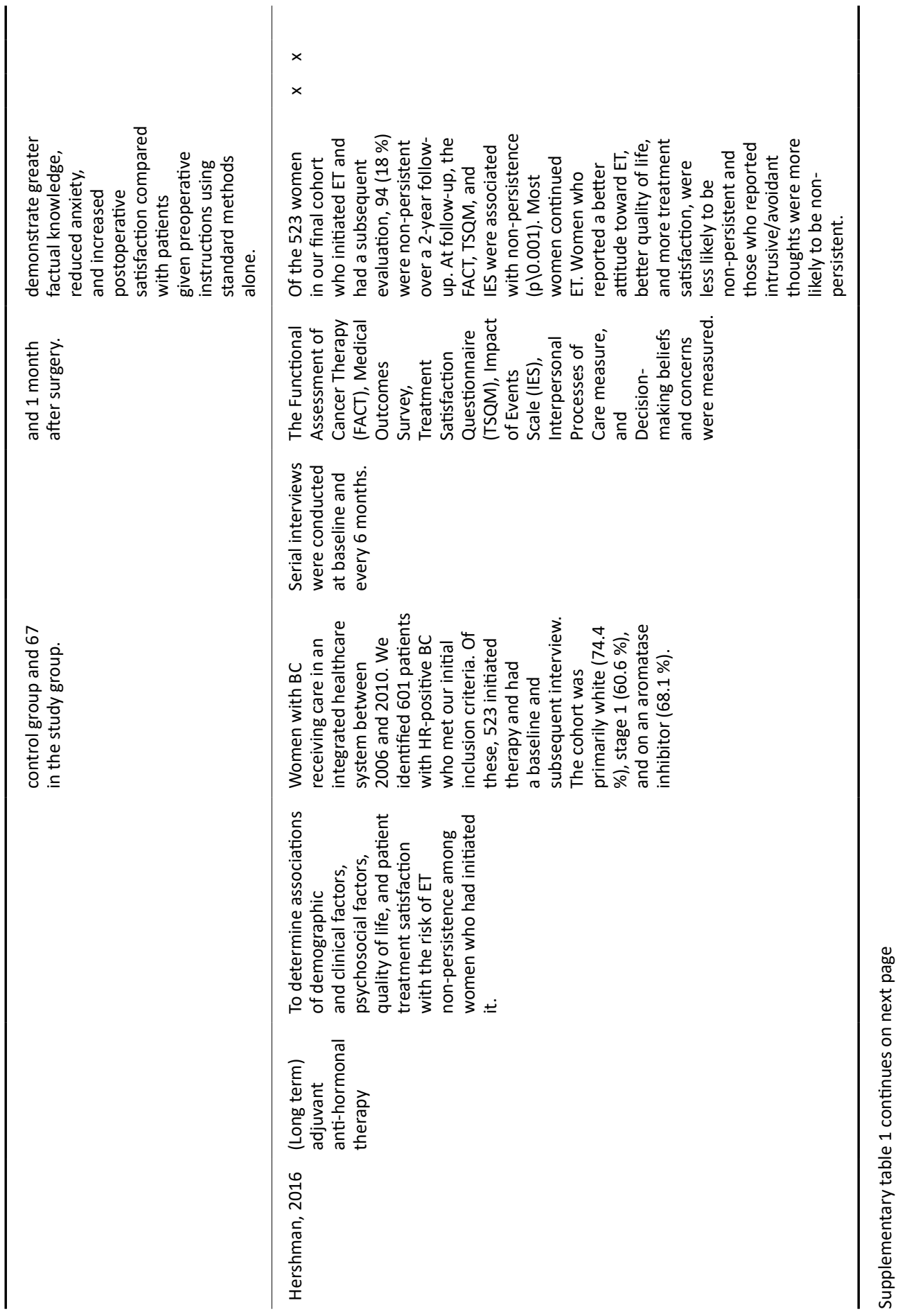




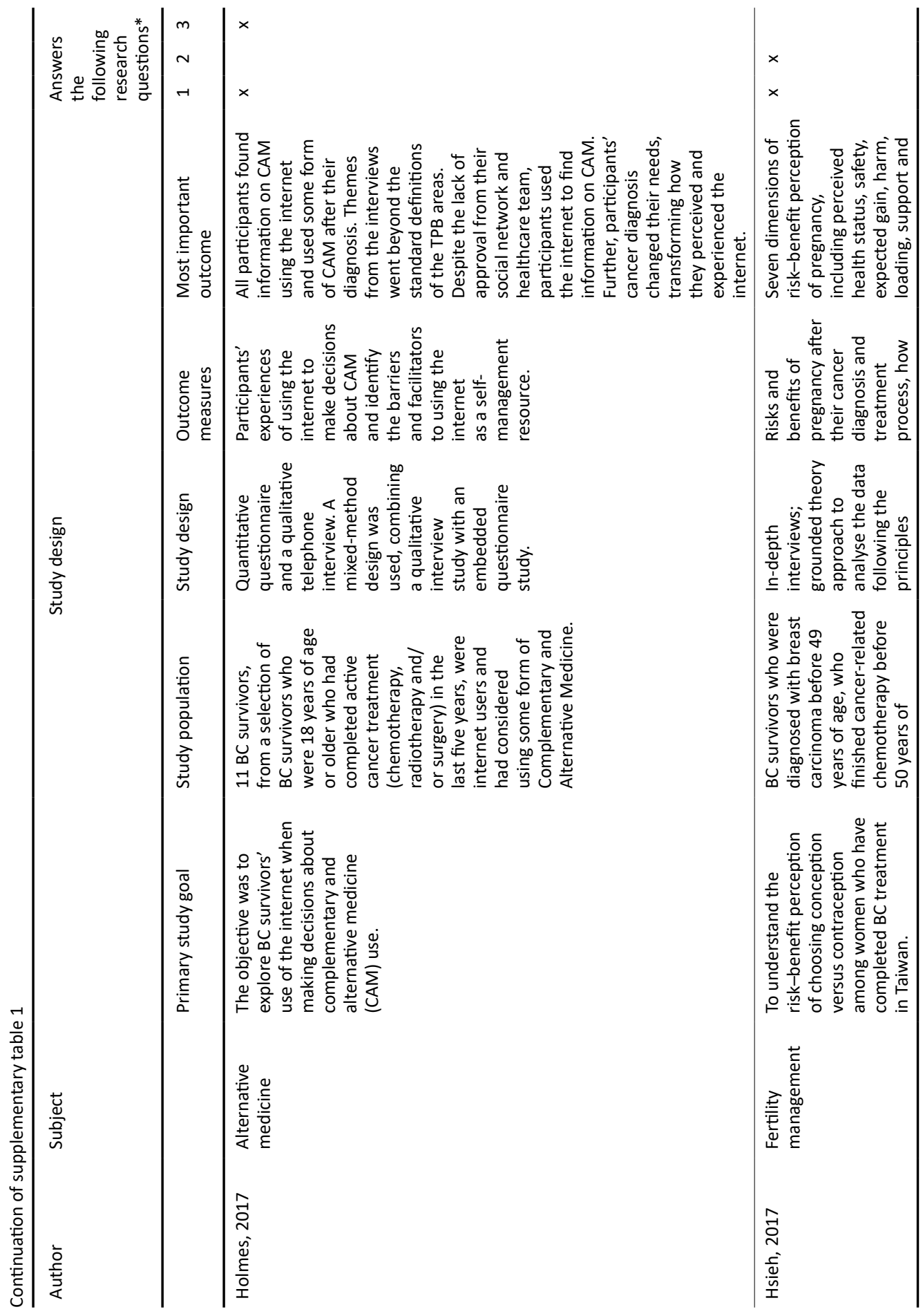




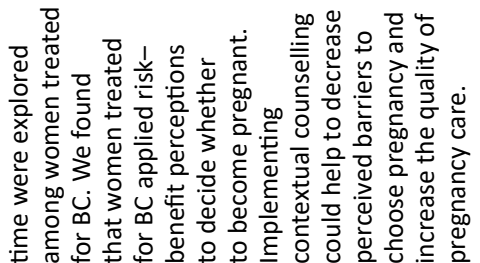

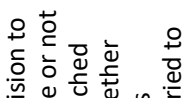

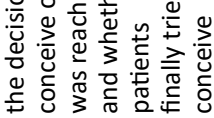

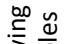

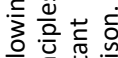

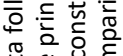

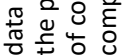

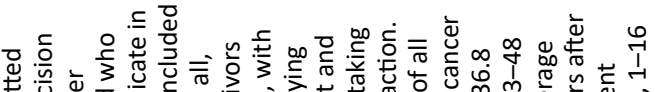

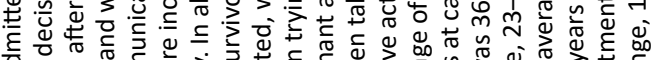

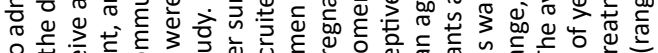

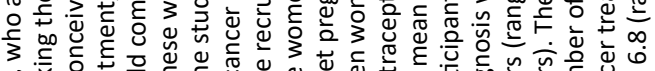

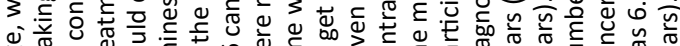

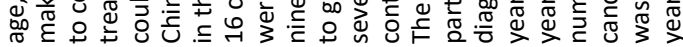

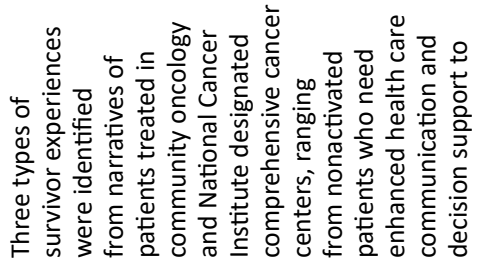

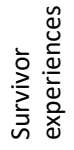

을

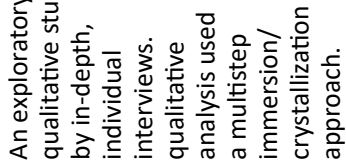

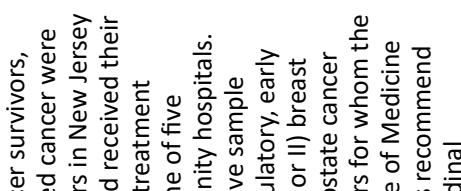

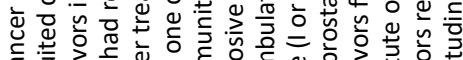

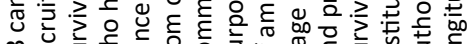

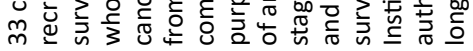

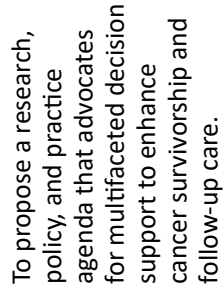

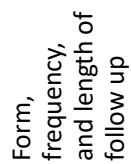

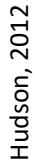



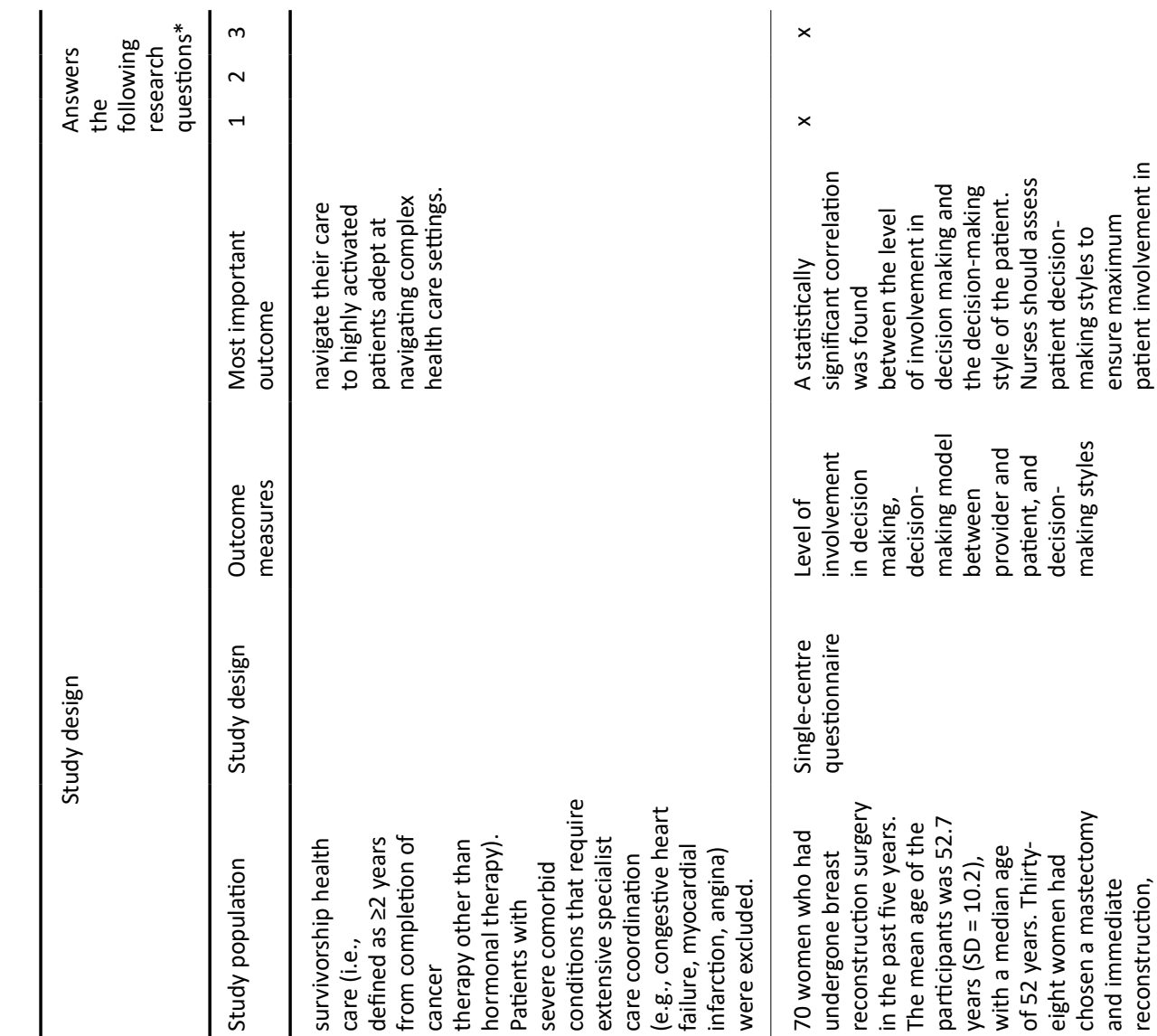

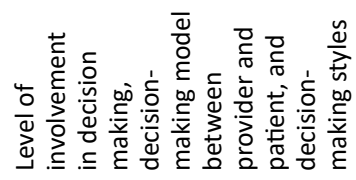

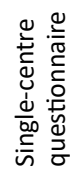

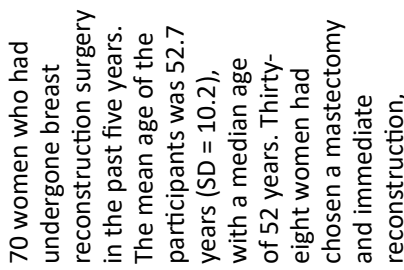

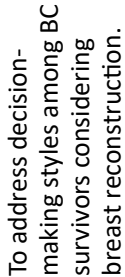

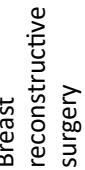

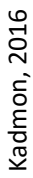




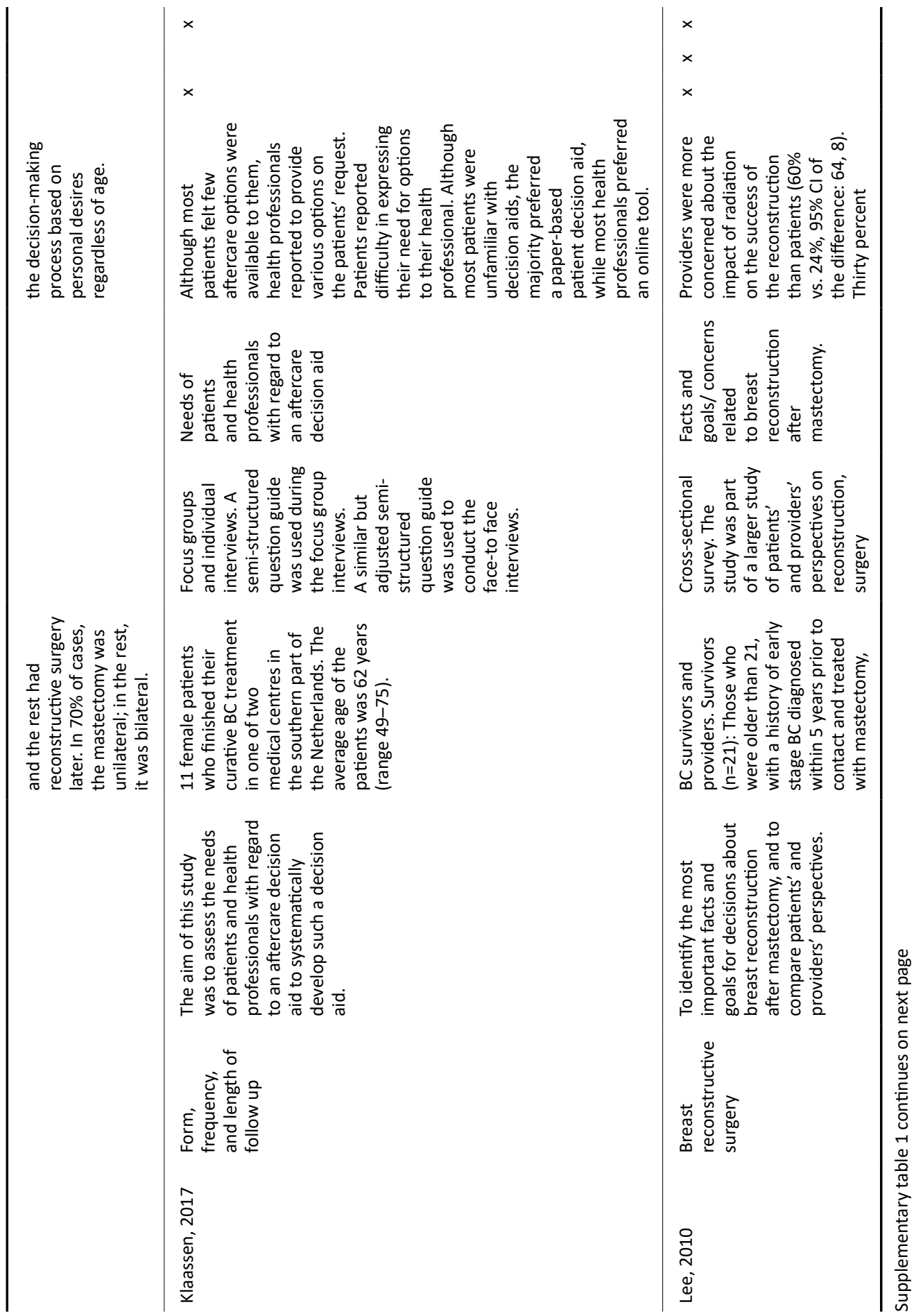




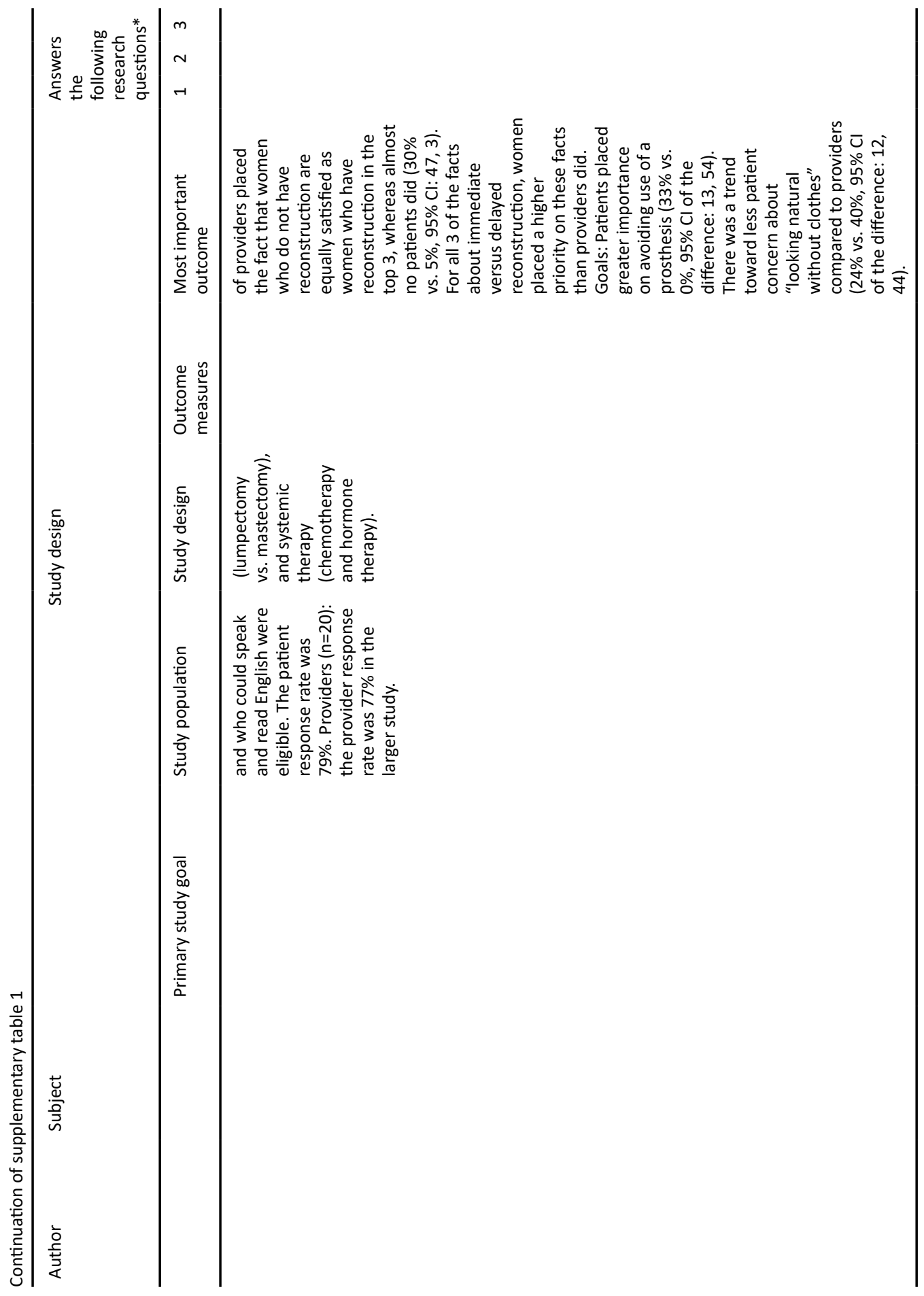




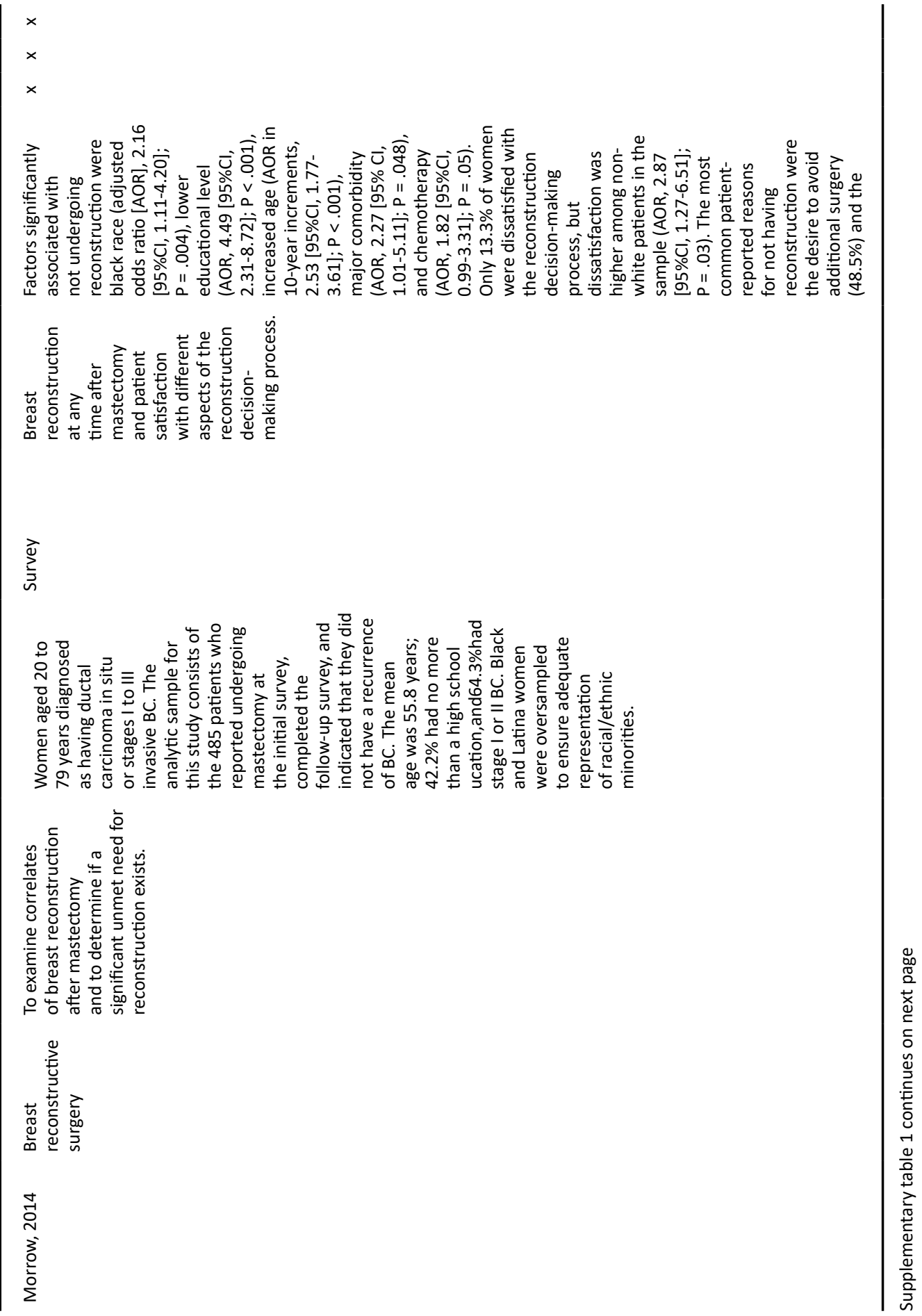




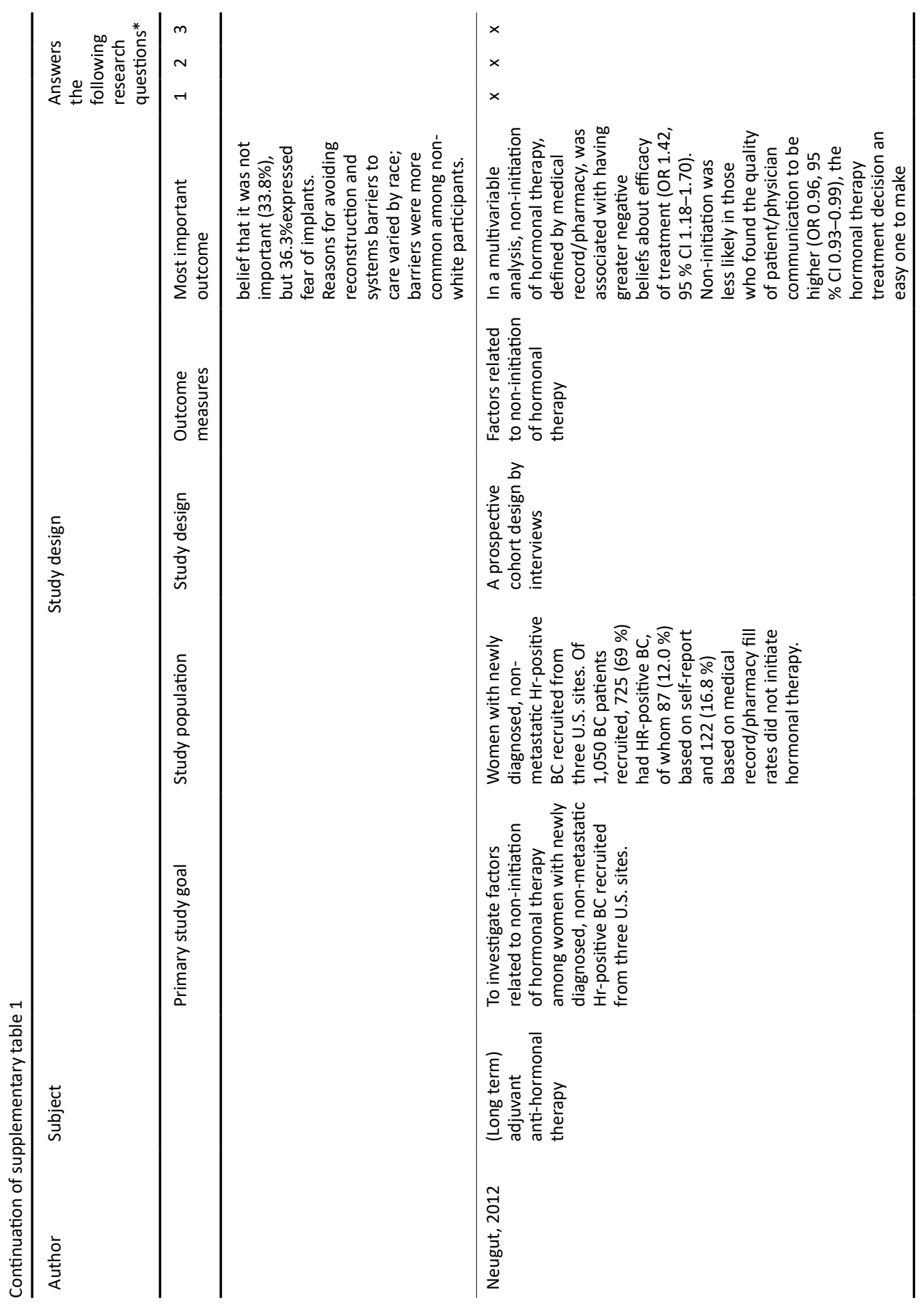




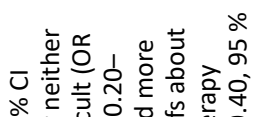

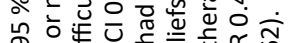

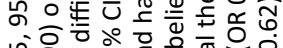

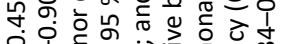
일

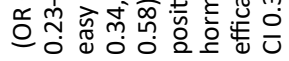

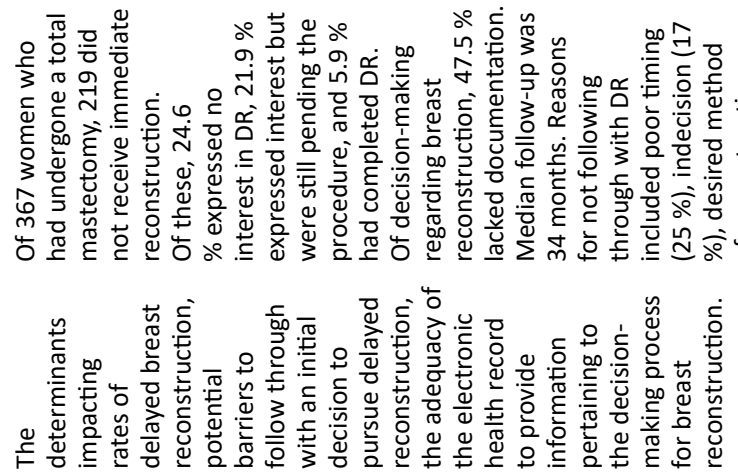

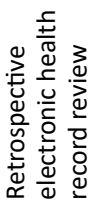

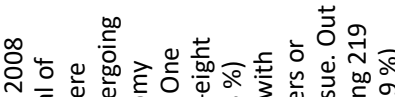

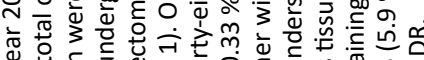

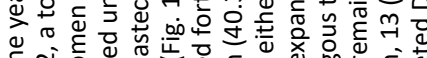

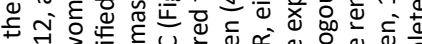

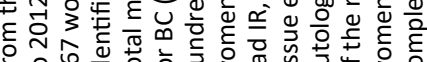

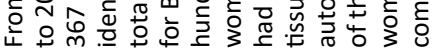

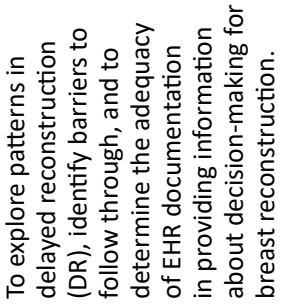

空

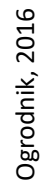




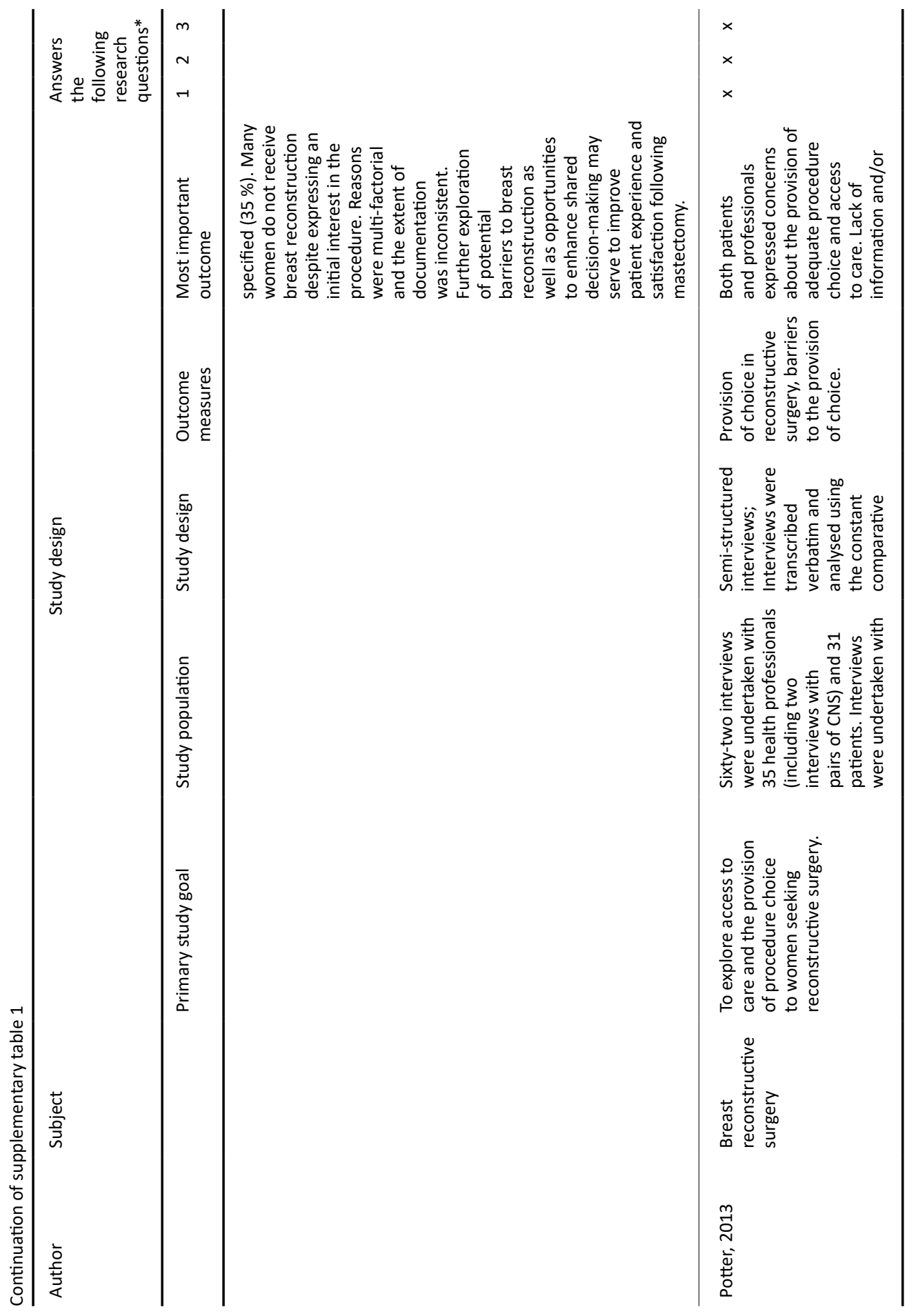



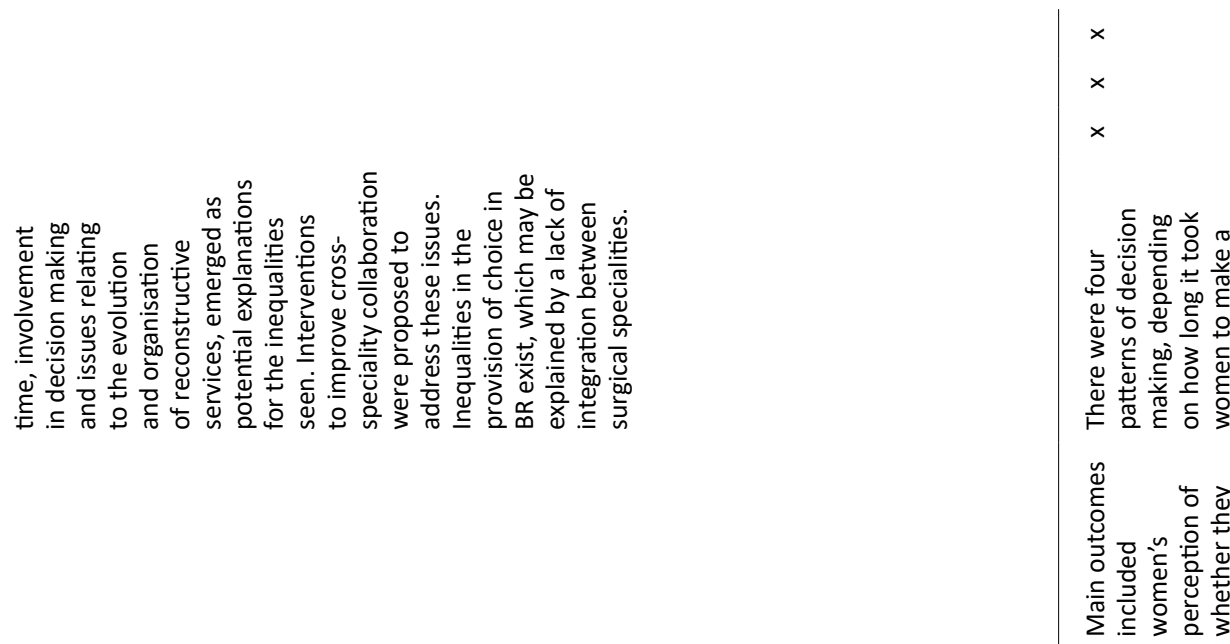

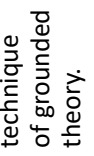

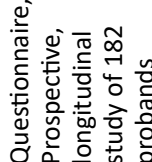

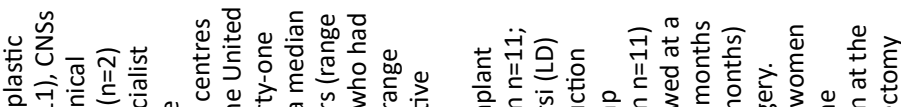

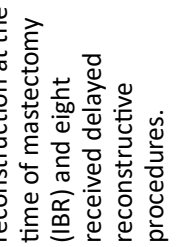

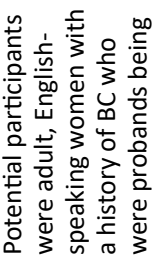
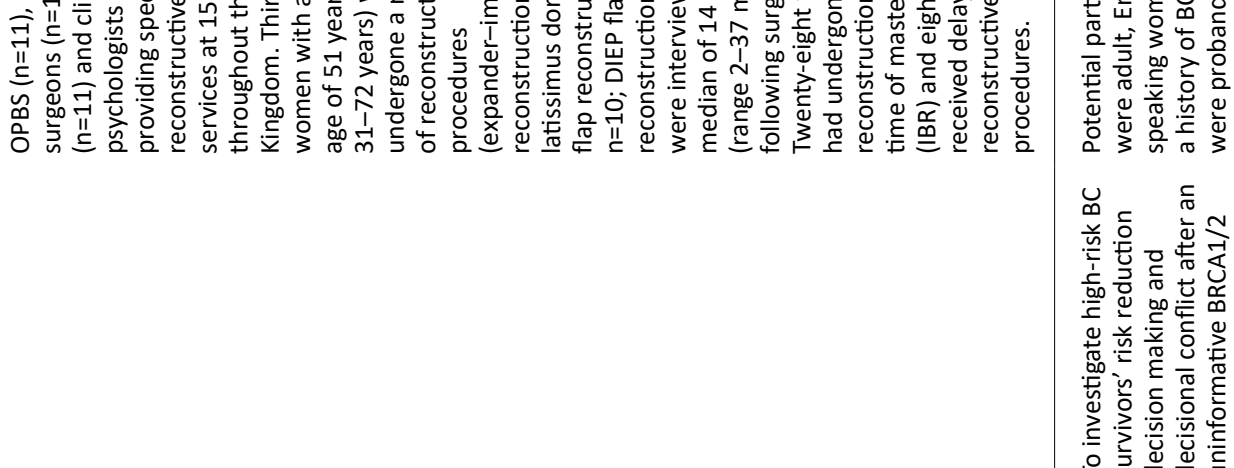

은

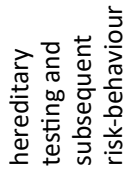

总 


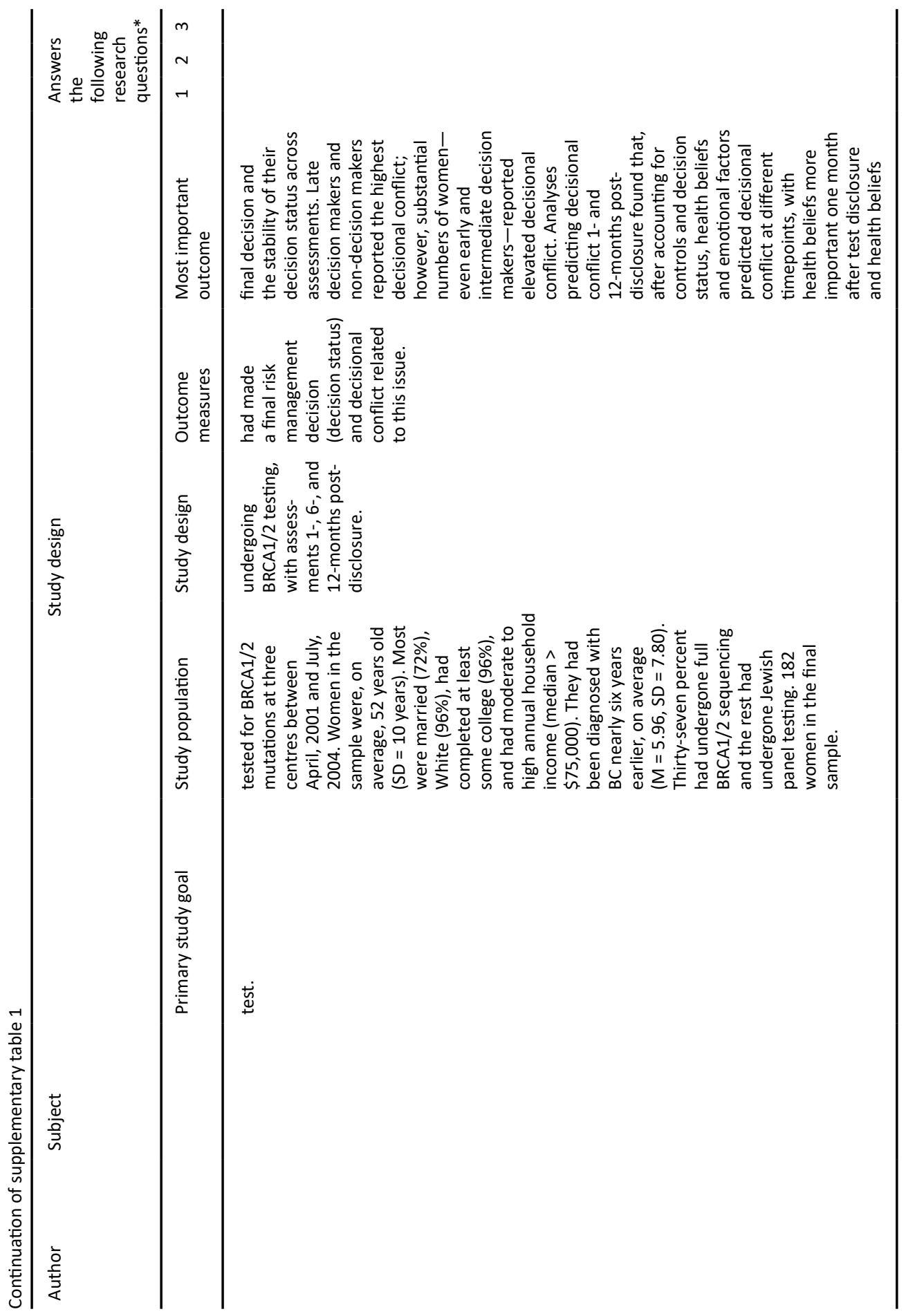




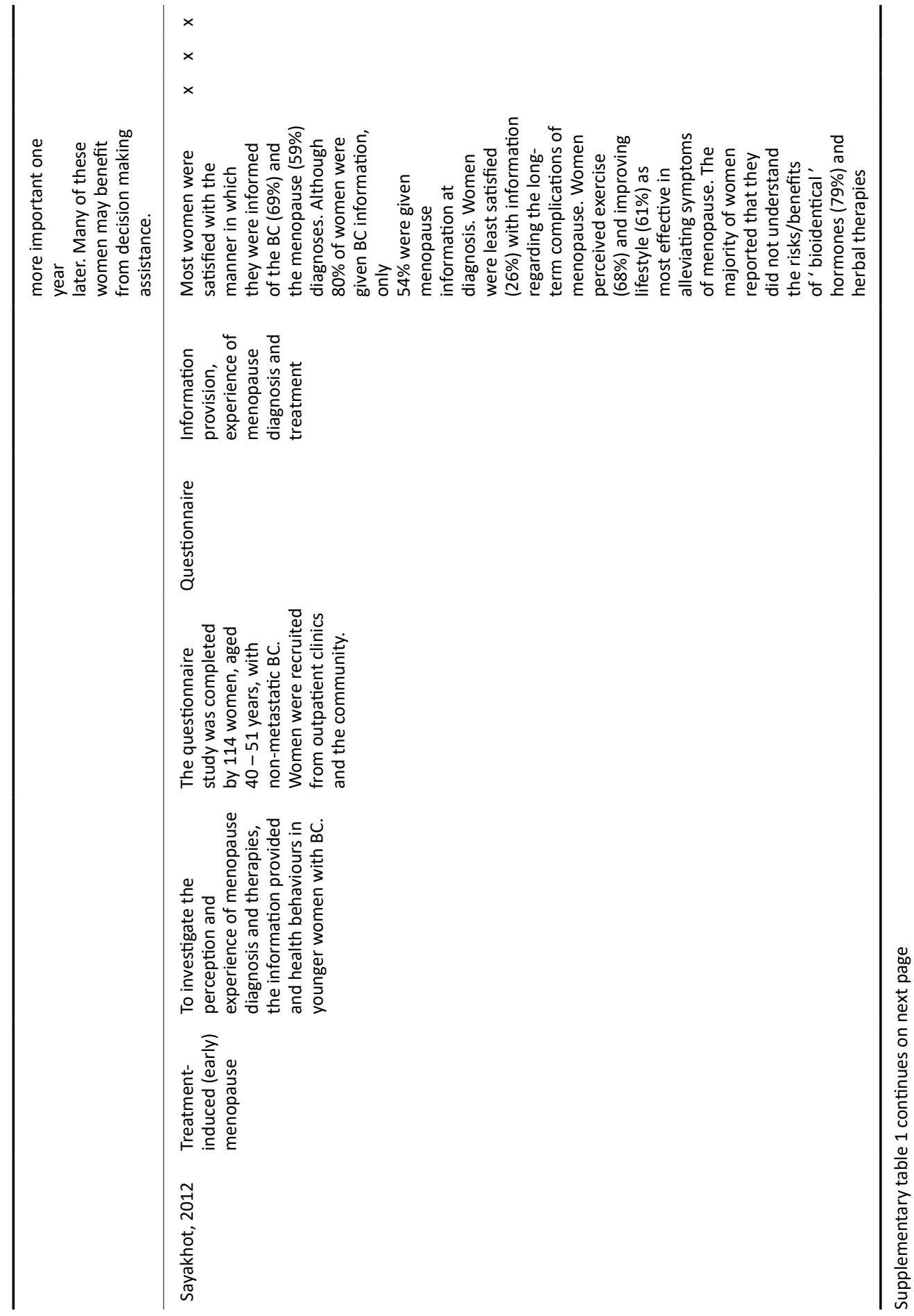




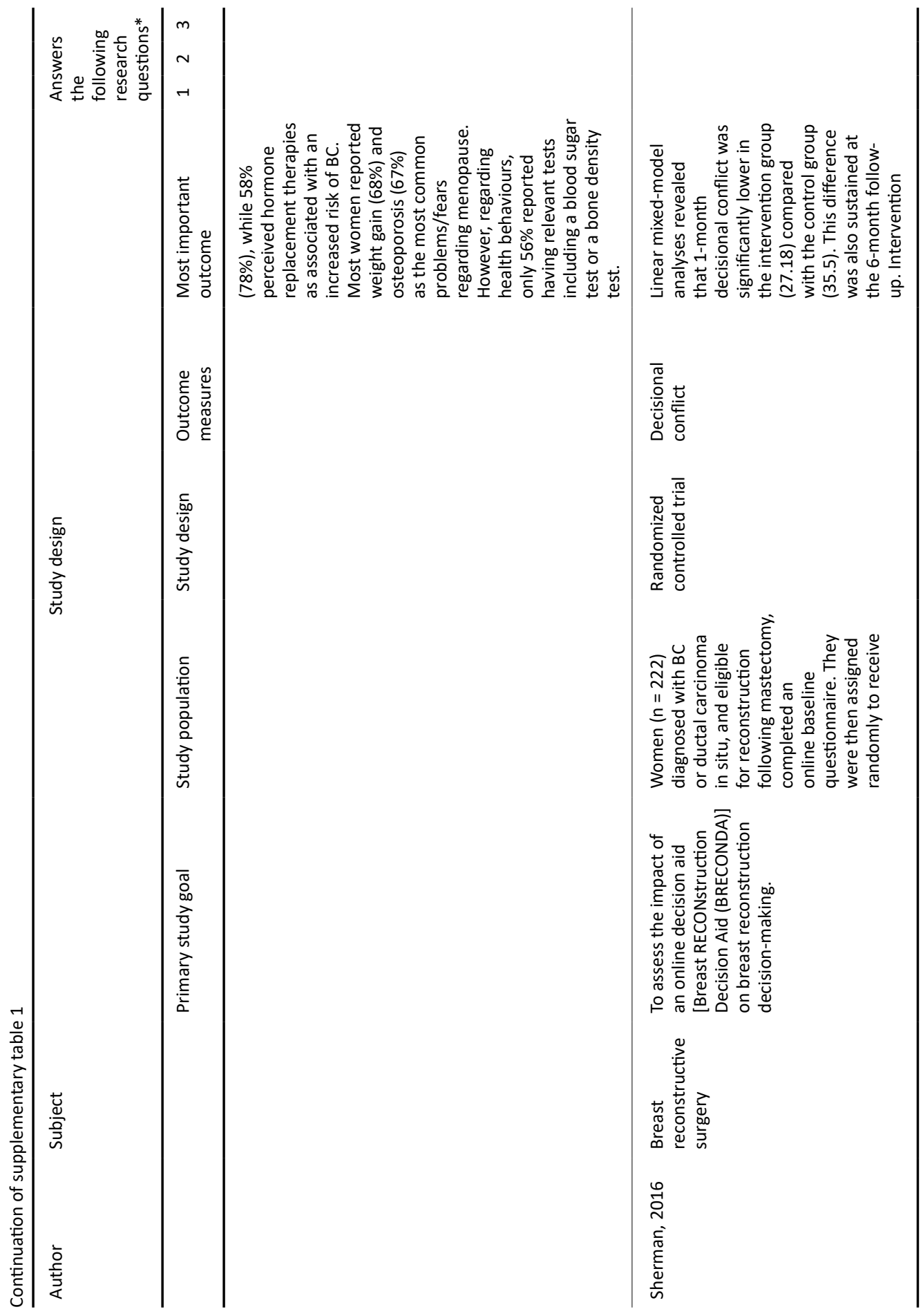



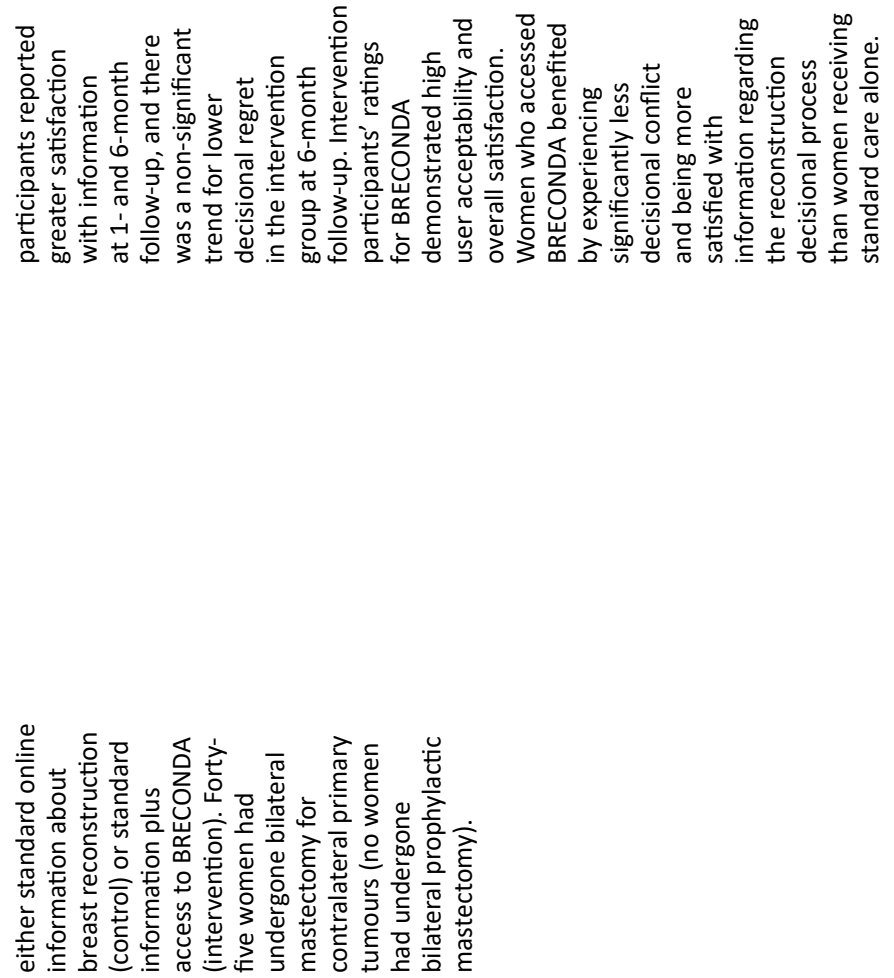

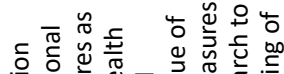

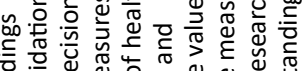

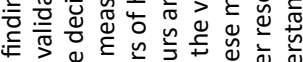

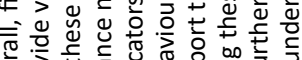

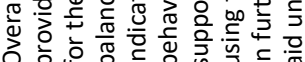

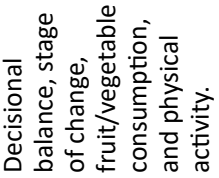

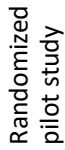

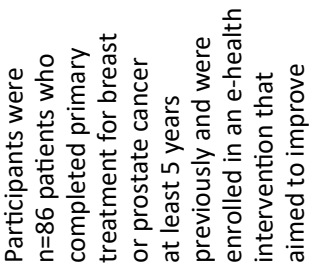

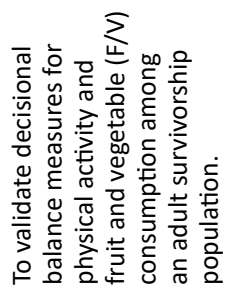

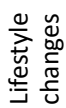

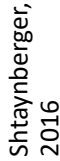




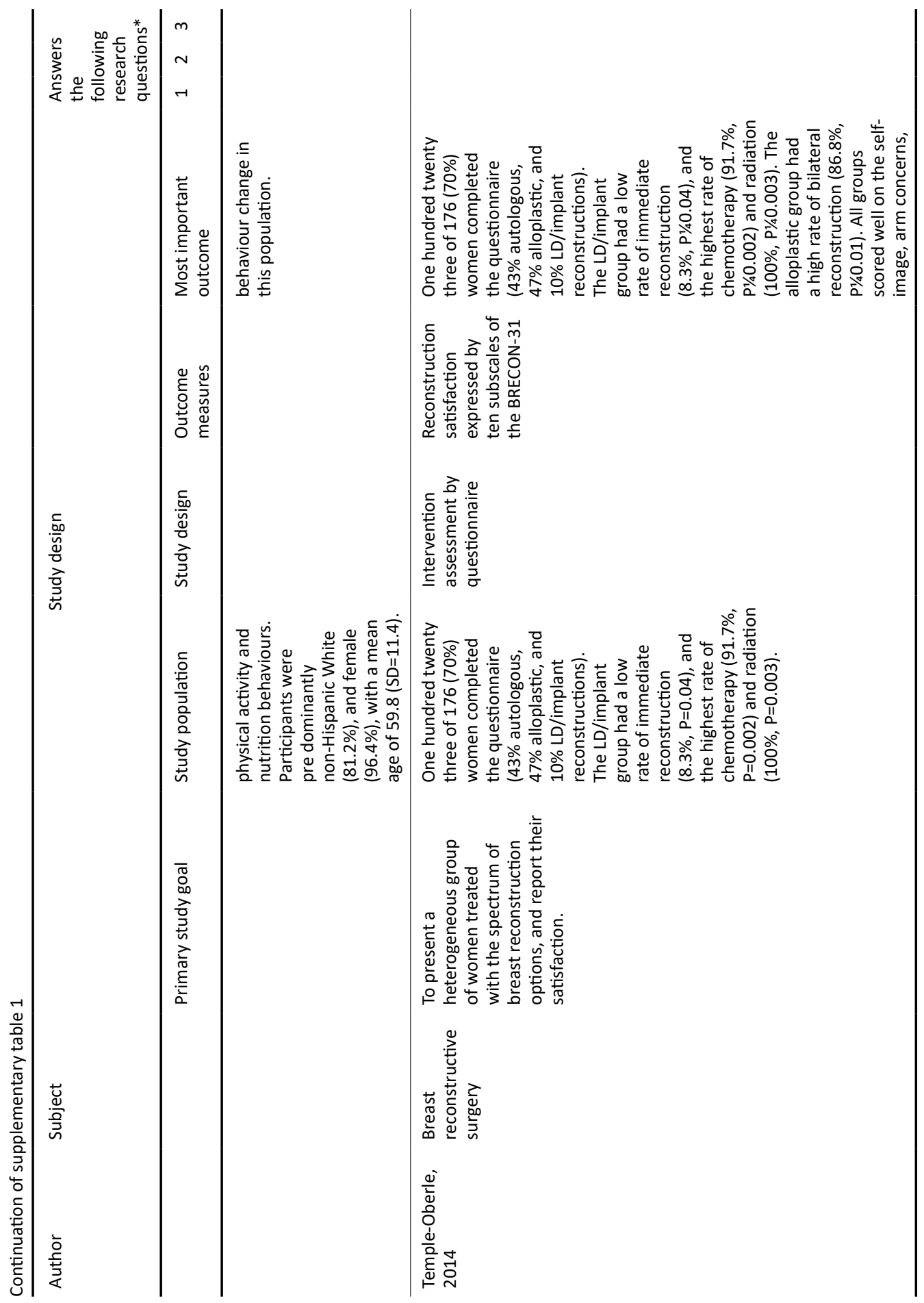



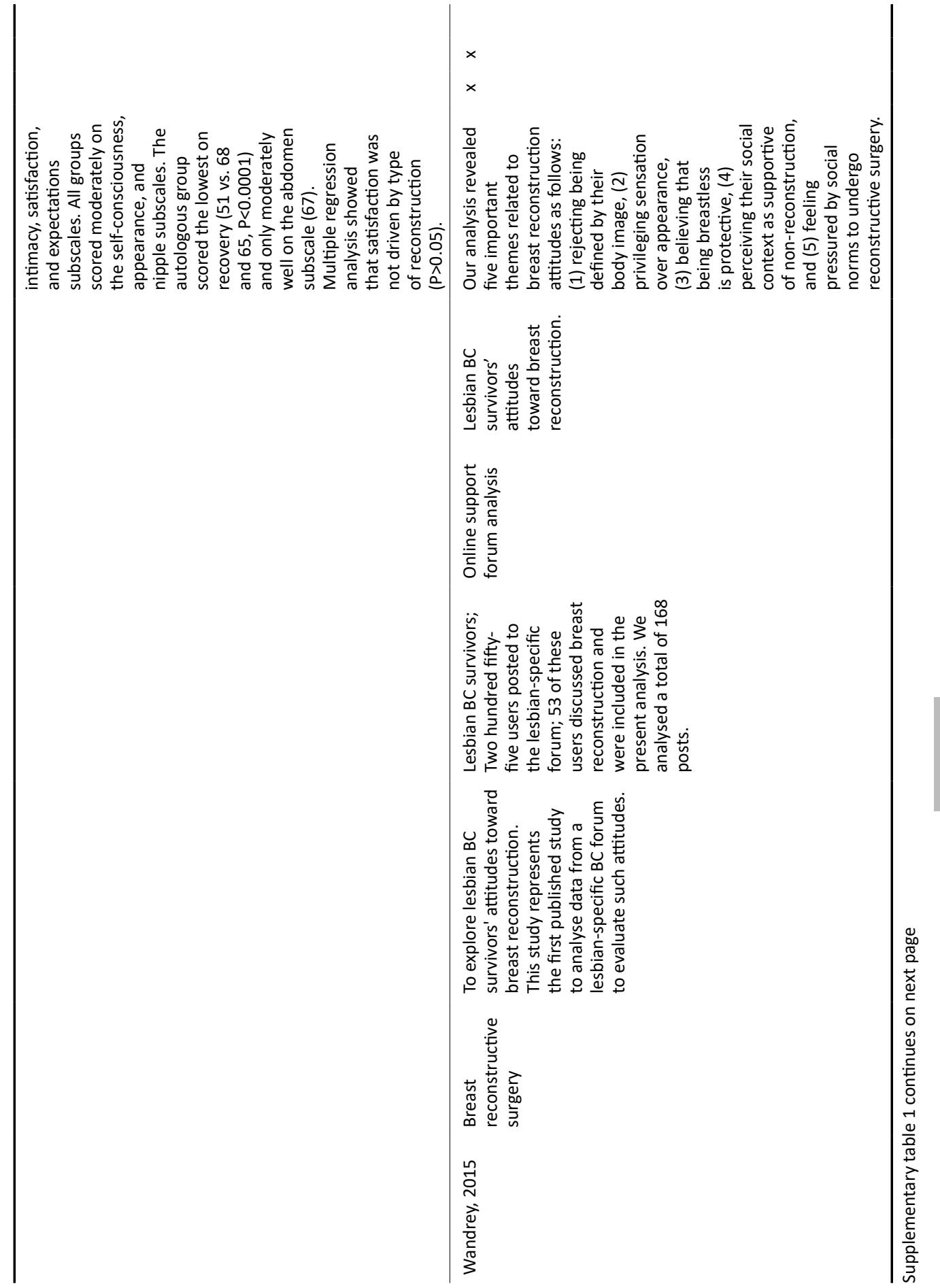


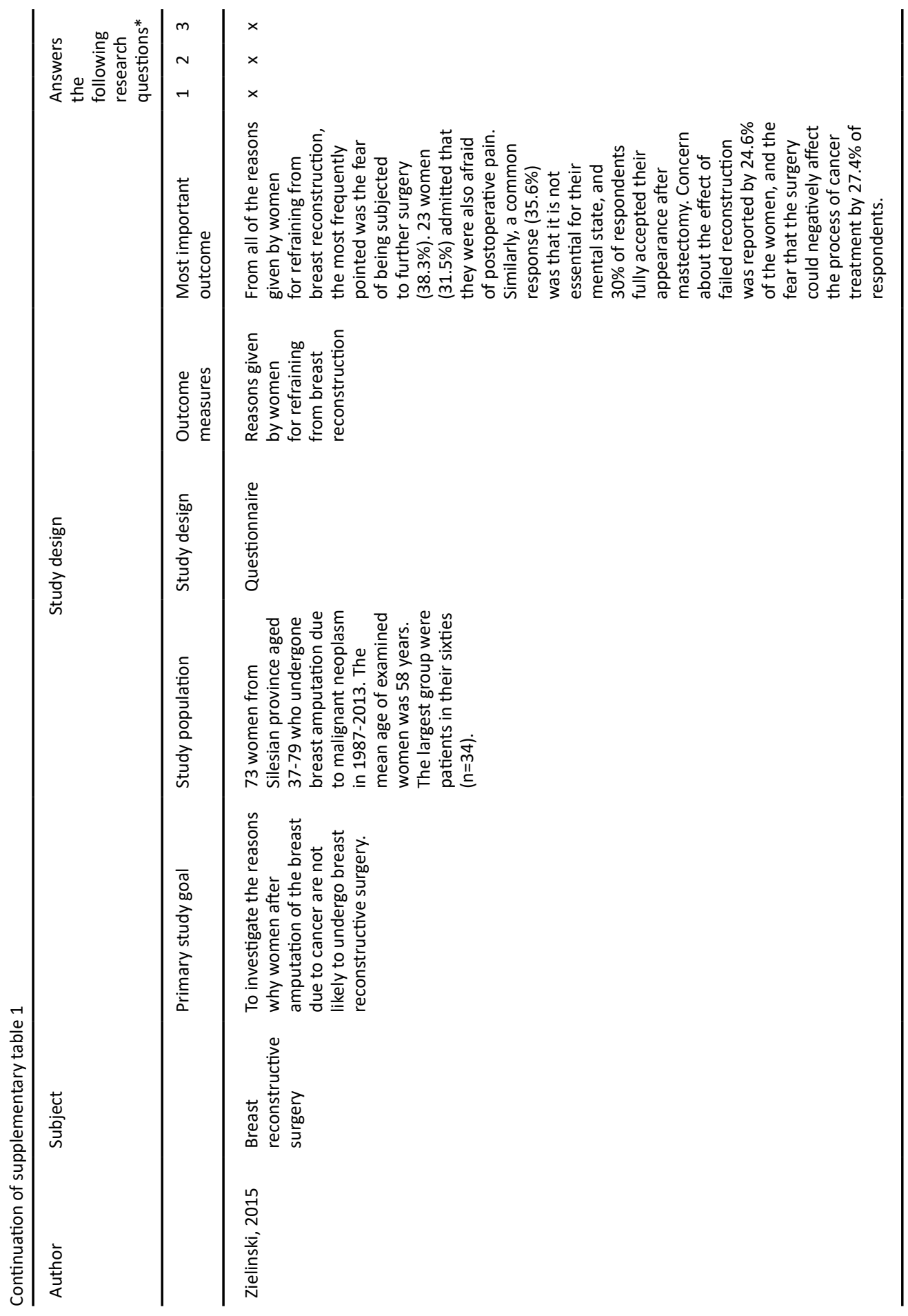




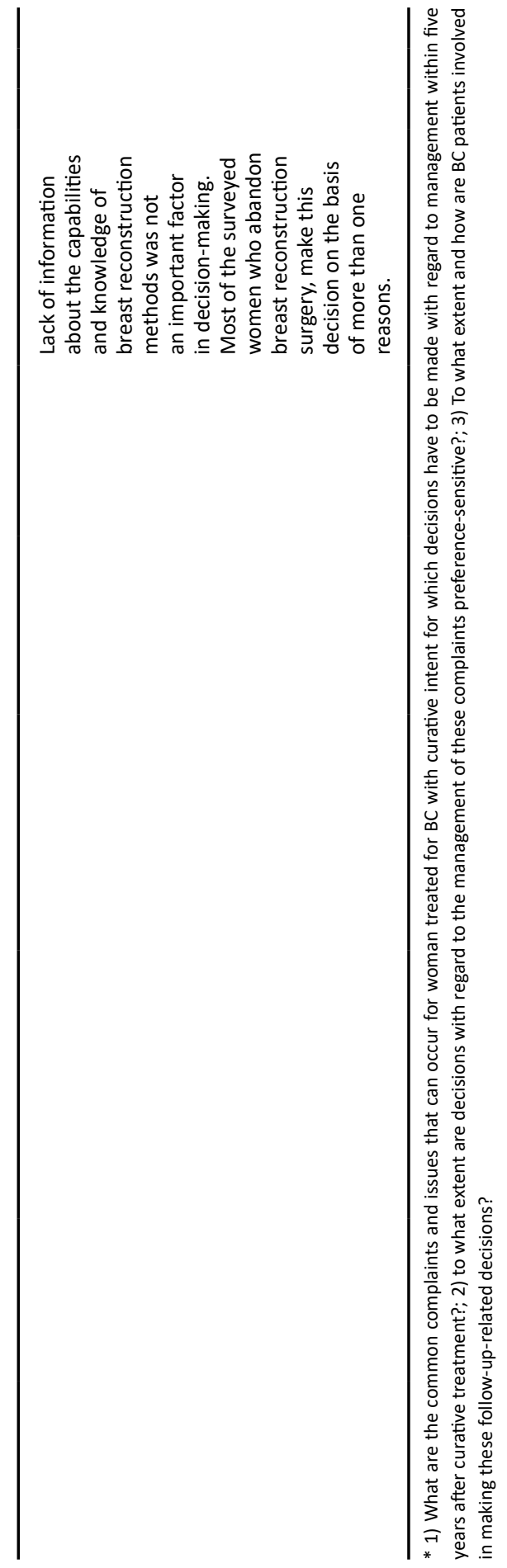




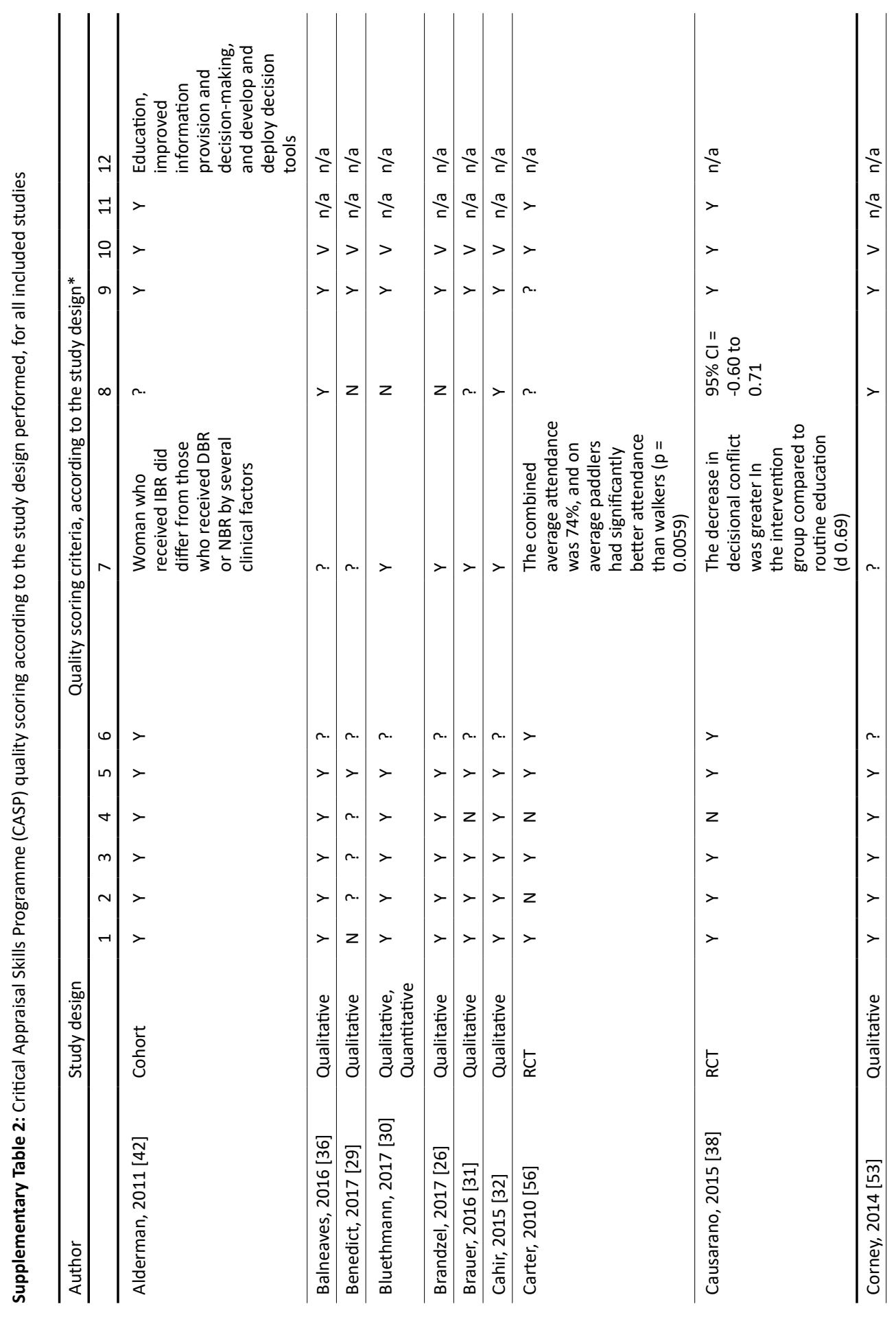




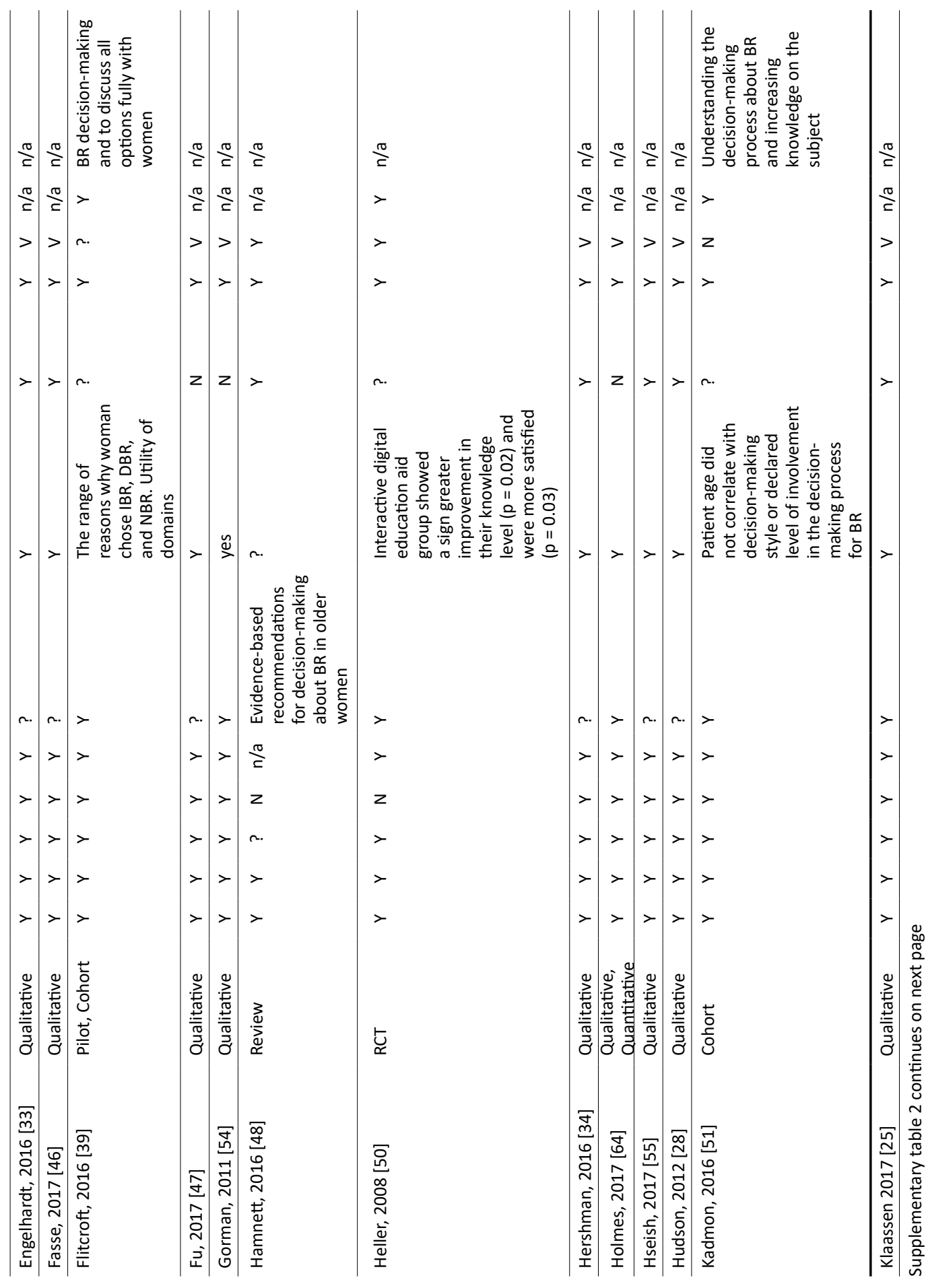




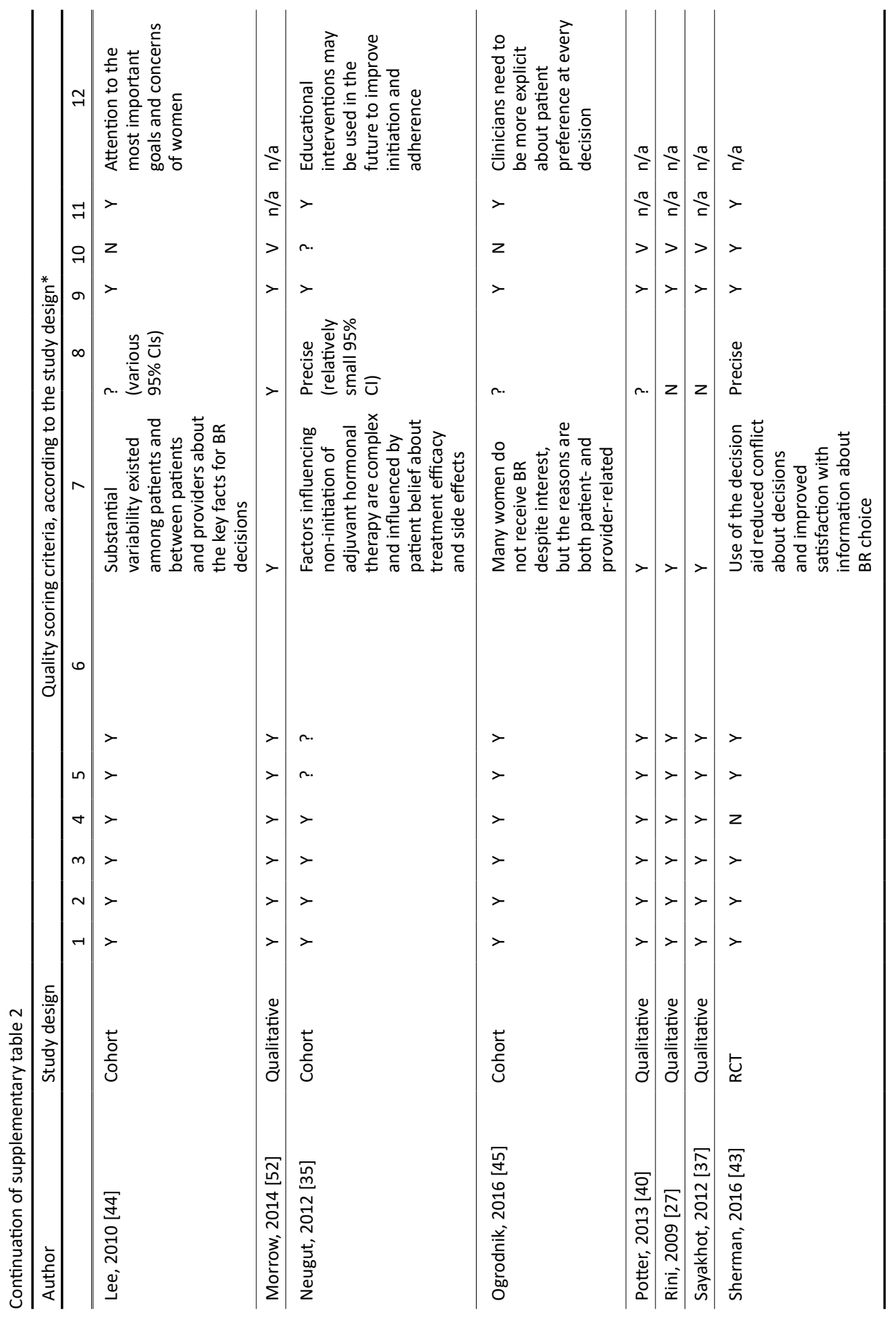




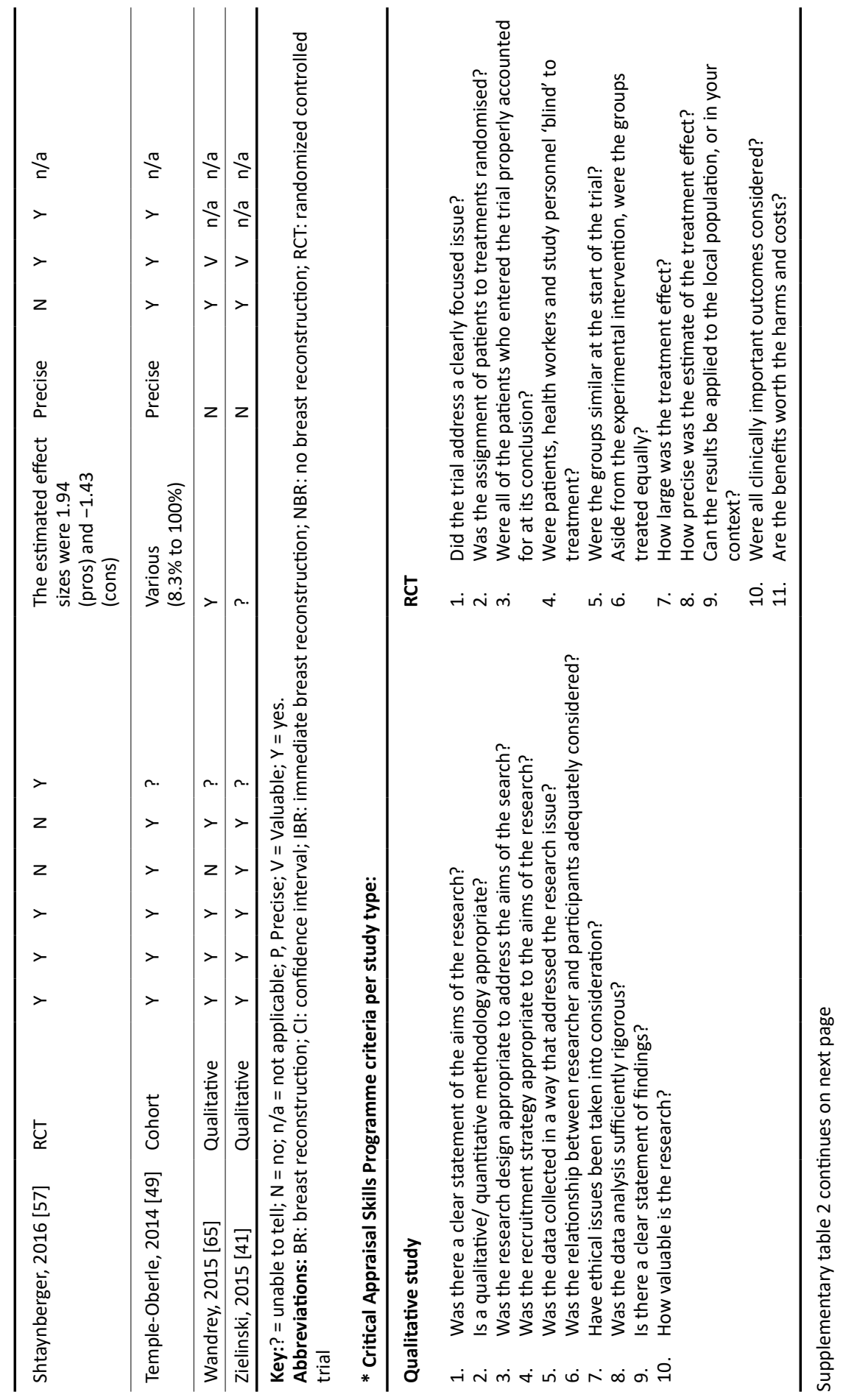




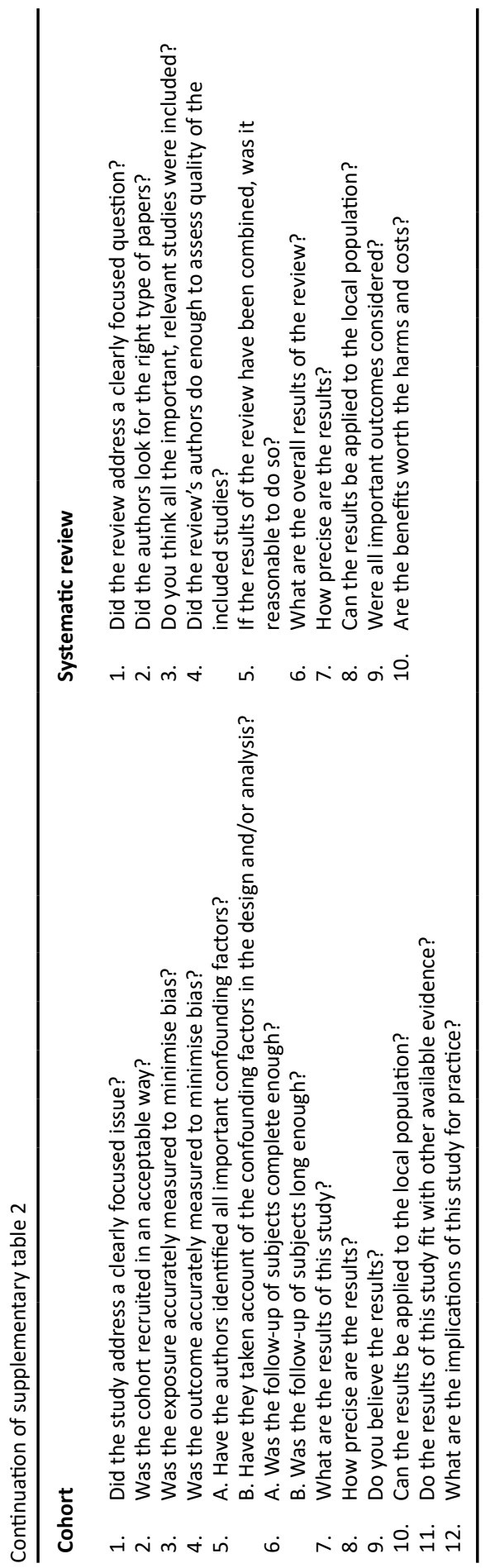





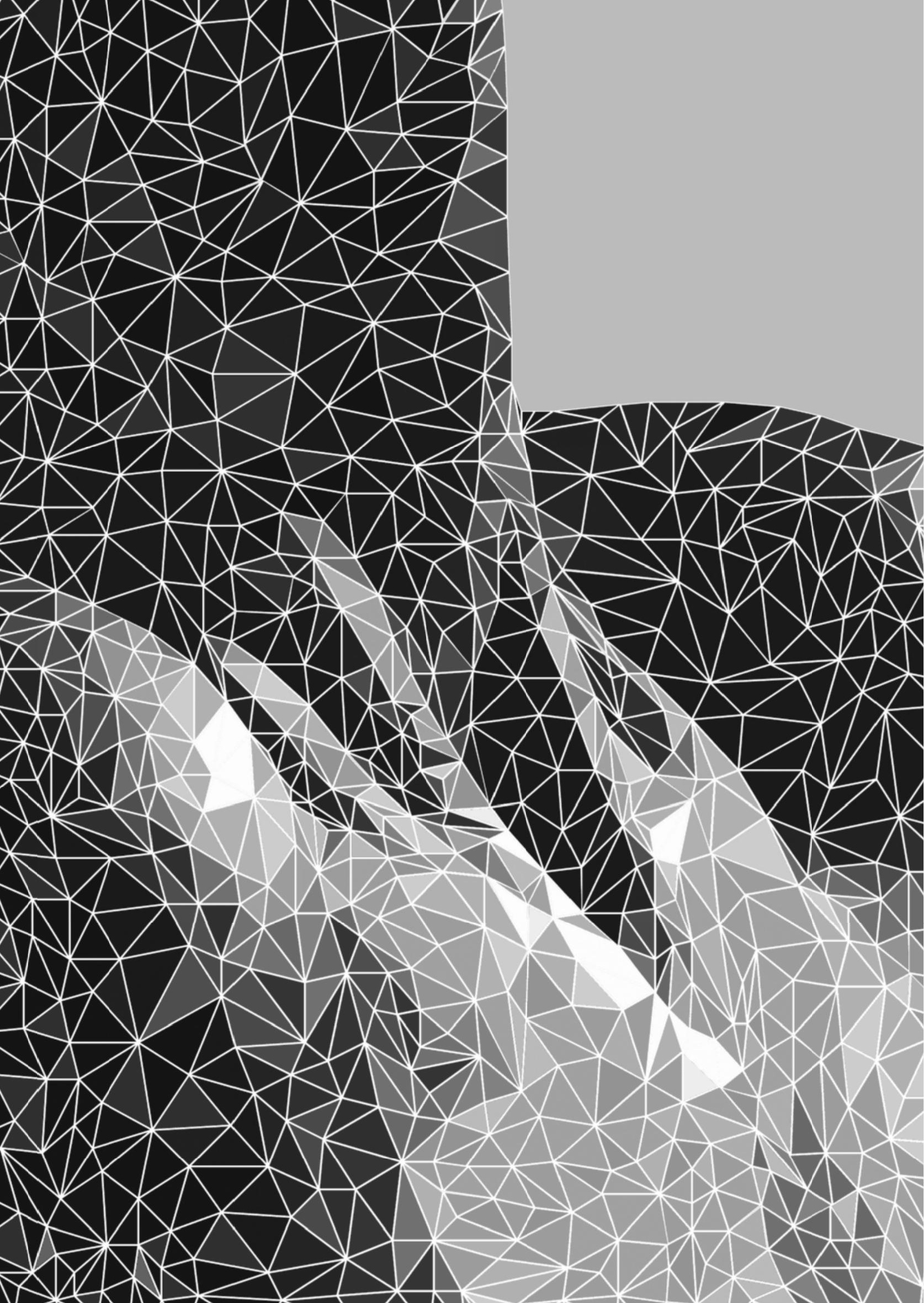


SUMMARY, CONCLUSIONS AND GENERAL DISCUSSION

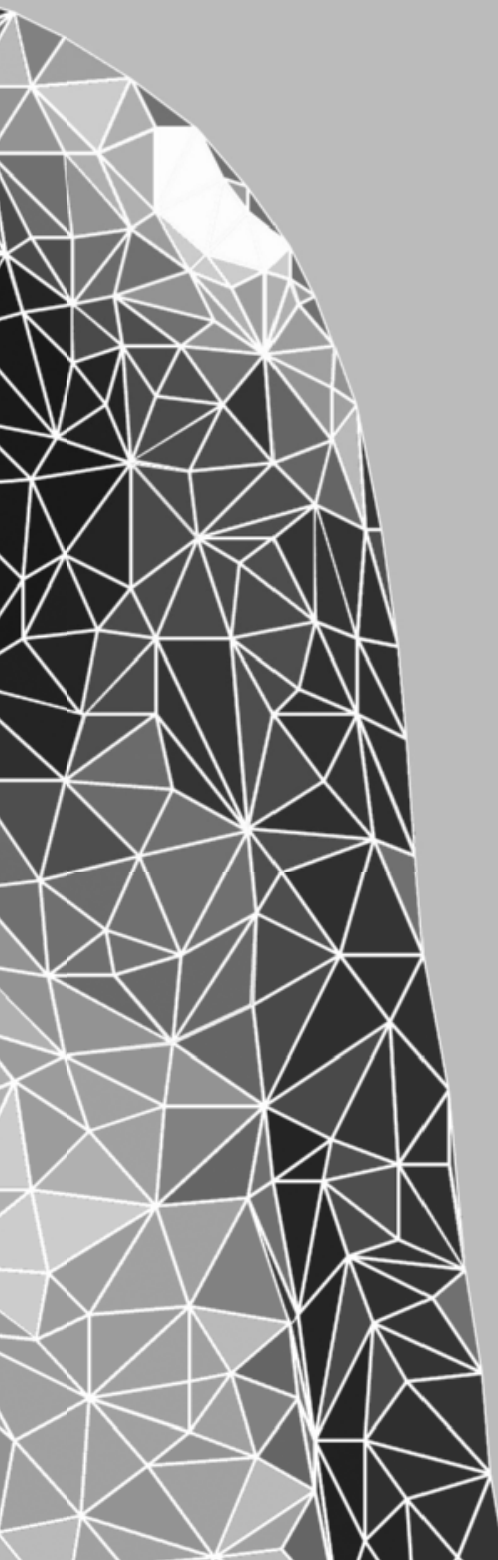





\section{Summary}

This thesis elaborates on aspects of variation in breast cancer treatment and follow-up care, focussing on the patient's perspective. The objective was to investigate whether patient preferences, needs and experiences with information provision and shared decision-making can explain the variation observed within the application of treatment modalities for breast cancer in the Netherlands, including chemotherapy, breast reconstruction, and follow-up care.

For all patient-reported surveys in this thesis, patients were selected from the Netherlands Cancer Registry (NCR). The NCR is a national database that documents population-based data about cancer incidence, diagnosis, and treatment [1], that includes all items for the NABON ('Nationaal Borstkanker Overleg Nederland') Breast Cancer Audit [2]. Patients were invited by the hospital administrations to complete the survey through the online PROFILES ('Patient Reported Outcomes Following Initial treatment and Long term Evaluation of Survivorship') Registry survey application [3]. Surveys consisted of validated Patient-Reported Outcome Measures (PROMs) such as the CTSO (Cancer Therapy Satisfaction Questionnaire) [4], EQ-5D-5L [5], EORTC-QLQ-C30/-BR23 [6, 7], and Breast-Q $[8,9]$, and were supplemented with questions about personal information including highest completed education, current treatment status, and comorbid disease. For the topics of chemotherapy timing and breast reconstruction, questions about information provision and shared decision-making were self-composed and included in the surveys. All responses were coded and merged with the clinical data available in the NCR. An exception from this is the study in Chapter 3, that was based on a surgical treated patient cohort from three hospitals in the Netherlands, who were invited by email to complete a questionnaire about their quality of life.

The patient-reported data were supplemented by several other sources. For the topic of chemotherapy timing, the clinicians' perspective was assessed through a survey distributed among 292 surgical and medical oncologists in the Netherlands. The survey consisted of questions about commonly accepted indications, contraindications, and considerations for the use of neoadjuvant chemotherapy in breast cancer patients. For the topic of follow-up care, a scoping literature review was performed to review the evidence for options and preferences that patients might have for decisions about follow-up care. Studies published between 2008 and 2017 were extracted from MEDLINE, PsycINFO, and Embase.

In the first part, the variation in timing of chemotherapy for surgically treated breast cancer patients in the Netherlands is discussed. Chemotherapy for early-stage breast cancer can be administered either before (neoadjuvant; NAC) or after (adjuvant; AC) surgical treatment. The potential advantages of chemotherapy for operable breast cancer in the neoadjuvant setting are tumour downstaging to enable breast conserving surgery $[10,11]$ and axillary conserving surgery [12], and assessment of the response to chemotherapy [10,13,14]. As subgroups of patients who achieve pCR after NAC have a better prognosis in terms of disease-free and overall survival [14], neoadjuvant chemotherapy should nowadays be considered as a preferred option in the treatment of high risk triple negative and HER2-positive breast cancer [15, 16]. In general, neoadjuvant chemotherapy could be recommended whenever adjuvant systemic therapy is 
indicated [17-19]. Although increasing over the past years, observed mean use of neoadjuvant chemotherapy was lower than for adjuvant chemotherapy [20]. In the Netherlands, $12 \%$ vs $31 \%$ of all newly diagnosed breast cancer patients in 2014 were treated with neoadjuvant and adjuvant chemotherapy, respectively, and a large between-hospital variation was found (0-97\%) [21]. As not all variation could be explained by patient and tumour characteristics [22], it was hypothesized that information provision was an underlying factor for this variation. We investigated this from both the perspective of the patient as the physician. For the study in Chapter 1, 400 breast cancer patients with stage II and III disease completed our survey about information provision and decisionmaking for chemotherapy timing. A minority of patients treated with adjuvant chemotherapy was informed about the possibility of neoadjuvant chemotherapy (stage II: $14 \%$, stage III: $31 \%$ ). Also, patients of both groups not always felt they had a choice in the timing of chemotherapy (stage II: $54 \%$ NAC vs $36 \%$ AC; stage III: $26 \%$ NAC, $54 \%$ AC). The physicians' perspective described in Chapter 2 was based on the responses of 138 surgical and medical oncologists from twothird of Dutch hospitals (response rate: $47 \%$ ). The results revealed that $75 \%$ of respondents regularly recommended neoadjuvant chemotherapy for downstaging to enable breast conserving surgery. Furthermore, $64 \%$ routinely recommended neoadjuvant chemotherapy when systemic therapy was indicated preoperatively. In contrast, some stated that breast conserving surgery after neoadjuvant chemotherapy increases the risk of an incomplete resection (21\%), surgical complications ( $9 \%$ ) and recurrence of disease (5\%).

In the second part, surgical treatment options for breast cancer and their impact on quality of life are discussed. The loss of a breast following mastectomy may negatively affect psychosocial health, body image, and sexual function [23]. For Chapter 3, almost 1900 surgically treated breast cancer patients from a cohort of patients from three Dutch hospitals completed a survey about quality of life, comprising EQ-5D-5L, EORTC-QLQ-C30/-BR23 and Breast-Q PROMs. The results demonstrate the importance of both breast conservation and reconstruction: patients with mastectomy reported the lowest mean quality of life based on the EQ-5D and the EORTC-QLQ-C30 'WellBeing' scales as compared to those treated with breast conservation or breast reconstruction. Guidelines recommend that the possibility of breast reconstruction should be discussed with every patient with an indication for mastectomy [17, 24, 25]. Furthermore, Chapter 3 demonstrates that each surgical procedure had its own favourable assets in terms of quality of life. Patients can be informed about these assets and align them with their preferences within the final decision about breast surgery. For instance, higher mean scores were found for every procedure other than mastectomy alone, including body image and arm functioning in patients with breast conserving surgery, and satisfaction with breasts, satisfaction with outcome, satisfaction with nipple, and sexual well-being in patients with autologous breast reconstruction. Yet, despite these positive effects of breast reconstruction, in 2014 a rather low mean rate of immediate breast reconstruction (IBR) of about $20 \%$ was found for patients undergoing mastectomy for primary invasive breast cancer in the Netherlands [26]. Furthermore, immediate breast reconstruction rates between Dutch hospitals ranged from $0-83 \%$ for ductal carcinoma in situ and $0-64 \%$ for invasive breast cancer, which could not be fully explained by tumour, patient, and hospital factors $[26,27]$. Thus, it was hypothesized that patient information and shared decision-making also could have played a role in this variation. Based on the survey responses of 510 out of over 1200 surgically treated 
breast cancer patients, we report in Chapter 4 that patients with immediate breast reconstruction significantly more often were informed about immediate breast reconstruction as a treatment option (IBR: 99\% vs no-IBR: 73\%), significantly more often discussed (dis)advantages with their physician (IBR: $86 \%$ vs no-IBR: $68 \%$ ), and significantly more often were involved in decision-making (IBR: 91\% vs no-IBR: 67\%) compared to patients without immediate breast reconstruction. Still, the final treatment decision was in line with the patient's preferences or clinical characteristics for four-fifth of patients. Being informed about immediate breast reconstruction, corrected for patient and treatment characteristics, increased the odds of receiving immediate breast reconstruction by fourteen times.

Finally, in the third part, the potential for (further) personalization of follow-up care after breast cancer treatment is explored. Through a survey administered among 876 surgically treated female patients diagnosed with early-stage breast cancer, health problems or symptoms that breast cancer patients may face during follow-up care and the effect of these symptoms on healthrelated quality of life were observed. The goals of breast cancer follow-up are to detect recurrent disease or new disease at an early stage, and to inform and counsel patients about the physical and psychosocial (late) effects of therapy [17, 24, 28, 29]. In total, 404 patients (46\%) responded to our survey. As reported in Chapter 5, almost all (93\%) respondents experienced health symptoms up to five years after diagnosis. Over $50 \%$ of all respondents experienced fatigue, psychological symptoms, and symptoms regarding the breast, and/or musculoskeletal, central nervous, and reproductive system. Besides, the majority of these health symptoms were reported consistently over time since diagnosis. Of interest, specifically treatment with chemotherapy was significantly associated with an increased risk of health symptoms, such as fatigue, respiratory, gastrointestinal, central nervous system, and skin symptoms. Furthermore, it is hypothesized in Chapter 6 that several of these health symptoms may be associated with a decrease in health related quality of life. Although the majority of health symptoms that occur after breast cancer treatment were associated with lower functioning of patients in daily life, specifically fatigue, musculoskeletal, gastro-intestinal, and symptoms of the central nervous system appeared to be associated with a decrease in global health and functioning. Breast cancer patients reported significant lower mean scores than the general population for role, cognitive, emotional, and social functioning. Besides, mean quality of life scores remained consistent regardless of time since diagnosis, and reporting multiple categories of symptoms resulted in significant lower mean quality of life. Finally, in Chapter 7, preferences and patient involvement in decisions about breast cancer followup are identified. This concerned decisions about surveillance for recurrent/secondary breast cancer, consultations for the physical and psychosocial (late) effects, recurrence-risk reduction by anti-hormonal treatment, and improving quality of life after breast cancer. Forty-one studies were included in the full-text analysis, that revealed that there was little evidence that physicians considered decisions about anti-hormonal treatment, menopausal symptoms, and follow-up consultations sensitive to patient preferences. However, decisions about breast reconstruction were regarded to be very sensitive to patient preferences, and patients were usually involved in decision-making. We suggest that further personalisation of follow-up care may lead to care of greater relevance and value to individual patients. 


\section{Conclusions}

On the one hand, treatment variation can be a result of personalised care, but on the other hand, it can also be an indication of lack of quality of care. The objective of this thesis was to investigate whether patient preferences, needs, and experiences with information provision and shared decision-making can explain the variation observed within the treatment modalities for breast cancer in the Netherlands, including chemotherapy, breast reconstruction, and follow-up care.

First, observed mean use of neoadjuvant chemotherapy was lower than for adjuvant chemotherapy, and a large between-hospital variation was observed in the Netherlands. We found that the need to make a treatment decision about the timing of chemotherapy was made explicit in only a small number of patients treated with adjuvant chemotherapy. In particular in stage II patients, information on the possibility of neoadjuvant chemotherapy was given to a minority of patients (Chapter 1). Furthermore, we found considerable variation in expert opinions about chemotherapy timing for early-stage breast cancer, possibly leading to differences in patient information (Chapter 2). We conclude that it is likely that an increase in consensus among physicians on the indications for chemotherapy timing could be reached through improved evidence-based guidance, which may subsequently improve patient information and thereby reduce treatment variation.

Second, we found that, in terms of quality of life, breast preservation and breast reconstruction have an added value for surgically treated breast cancer patients (Chapter 3 ). Despite that, rather low immediate breast reconstruction rates were observed in Dutch hospitals with a betweenhospital variation, ranging from $0-83 \%$ for ductal carcinoma in situ and from $0-64 \%$ for invasive breast cancer. We found that patients who had received immediate breast reconstruction had been informed better about immediate breast reconstruction (including advantages and disadvantages) and felt significantly more often involved in shared decision-making than patients who had not received immediate breast reconstruction. Still, the final treatment decision was in line with the patient's preferences or clinical characteristics for around $80 \%$ of patients. Therefore, we conclude that the majority of observed treatment variation was likely the result of personalised care. Providing sufficient information on all relevant treatment options was found to be crucial: the odds for opting for immediate breast reconstruction increased fourteen times when patients received adequate information about the possibilities for breast reconstruction (Chapter 4). As breast preservation and reconstruction options each had their own favourable assets in terms of quality of life, information provision and decision-making could be refined by aligning these assets with patient preferences (Chapter 3). By that, optimal personalisation of care could be reached.

Third, there was a demand for personalised follow-up care for breast cancer patients. Up to five years after diagnosis, almost all (93\%) breast cancer patients experienced health symptoms. A prevalence of over $50 \%$ was found for fatigue, psychological symptoms, and health symptoms regarding the breast, and/or musculoskeletal, central nervous, and reproductive system. The majority of these health symptoms were consistently present regardless of time since diagnosis. Treatment with chemotherapy was associated with an increased risk of health symptoms (Chapter 5). Furthermore, breast cancer patients up to five year after diagnosis demonstrated on average a 
social, cognitive, emotional, and role functioning worse than the general population. Although the majority of health symptoms was associated with either one of the functioning scores, specifically fatigue, gastro-intestinal symptoms, musculoskeletal symptoms, and symptoms of the central nervous system were significantly associated with a decrease in several functioning domains (Chapter 6). We conclude that follow-up and research towards effective interventions could best focus on prevalent symptoms that have a large impact on quality of life. However, we also found that patients are currently not involved in all decisions about content or form of follow-up that affect them during follow-up (Chapter 7). It is expected that, with more guidance in follow-up and more attention to patient preferences and needs, follow-up can become more personalised with consequent reduction in unwanted variation.

In general, sufficient patient information and shared decision-making seem key in providing treatment and follow-up care for breast cancer that is in line with individual patient preferences. By that, variation will be the result of good quality of breast cancer care. This may lead to improvements in quality of life in breast cancer patients. 


\section{General discussion}

\section{Interpretation of the results}

Treatment variation is the logical consequence of the availability of more than one treatment option. Evidence-based guidelines present recommendations for accepted treatment and followup options to physicians and patients and form the basis for medical decision-making. Preferably, the final treatment decision is in line with patient characteristics, needs, and preferences; in other words, personalized care is provided, resulting in treatment variation. However, when treatment variation is observed, this can also be an indication of unequal access to or a lack of quality of care. Therefore, insight in underlying causes of variation is needed to conclude which of both explanations is most likely. In this thesis, between-hospital treatment variation was observed in the use of both neoadjuvant chemotherapy and immediate breast reconstruction, which could not be (fully) explained by patient, tumour, and hospital characteristics [21, 22, 26, 27]. We hypothesised that underlying processes of information provision and decision-making as well as patient experiences could provide further insight. Our findings about immediate breast reconstruction indeed illustrate variation as a result of personalised care: four-fifth of patients reported that the final treatment decision about immediate breast reconstruction was in line with her preferences or contra-indications as presented by her physician [30]. Furthermore, decisions about breast reconstruction were considered to be very sensitive to patient preferences, and patients were usually sufficiently involved in the decision-making process [31]. Thus, one expects that final treatment decisions are in line with patient preferences. In contrast, our findings about the variation in the use of neoadjuvant chemotherapy suggest unequal access to care, as shortcomings in information provision and shared decision-making were found [32]. This, however, requires more nuance, because we also found that a considerable variation existed in expert opinions about chemotherapy timing for early-stage breast cancer [33]. A possible explanation for this is that evidence for treatment risks and benefits of neoadjuvant chemotherapy still is preliminary $[15,16]$. This suggests that the cause of under-informing patients may be a result of a lack of evidence-based information, rather than the lack of incentive to properly inform patients or to include them in the decision-making process [34].

Still, we think that there is room for improvement in making informed decisions about breast reconstruction and timing of chemotherapy. In order for a breast cancer patient to make an informed treatment decision, she needs to be aware that there is a decision to be made, she needs to receive the most up-to-date evidence-based information about the risks and benefits of each treatment option, and her preferences should be incorporated in the final treatment decision [35, 36]. For breast reconstruction decision-making, insufficient, inadequate, or unclear information is known to be associated with decisional regret [37]. We found a discrepancy in terms of information provision about immediate breast reconstruction between patients who had and had not received immediate breast reconstruction [30], indicating a need for improvement to prevent decisional regret in individual breast cancer patients. Furthermore, the need to make a decision about the timing of chemotherapy was found to be made explicit in only a small number of adjuvant treated early-stage breast cancer patients, which was confirmed by the literature [38]. 
This could explain that respondents of both groups not always felt to have had a choice in the timing of chemotherapy [32].

The rationale for personalised care can be found in the ideas of value-based healthcare, which aims to improve care quality by adding value to an individual patient's treatment, and thus making care more personalised $[39,40]$. For decisions about follow-up care, the potential for personalisation of care was the starting point for all studies in the third part of this thesis. Follow-up aims to detect recurrent or new disease at an early stage, and to inform and counsel patients about the physical and psychosocial (late) effects of therapy [17, 24, 28, 29]. However, guideline recommendations for follow-up are rather generic for the breast cancer population, and not personalised towards the characteristics of individuals or sub-groups of patients. For the early detection of recurrent disease, surveillance imaging schemes are universal for curatively treated patients, even though patients have an individual risk of recurrence [41]. Here, we currently expect little variation, while more variation in surveillance schemes may lead to detection which is better aligned with individual clinical needs. For the detection and management of late (treatment-induced) effects of breast cancer, general recommendations are made, even though the confrontation with breast cancer in terms of (treatment) consequences may be a highly individual experience [42, 43]. A large treatment variation is expected that is not per se the result of personalised care, but rather due to a certain arbitrariness.

Currently, the arrangements for follow-up care suboptimally meet the needs of patients with breast cancer, and there is a growing demand for personalised care planning [44-47]. First, towards the personalisation of surveillance, we believe efforts should be made to improve patient understanding of the goals of surveillance [48]. As we identified in our scoping review, patients generally want more frequent surveillance, even though there is no evidence of effectiveness to justify this [49]. Second, there is a great difficulty in predicting which patients will encounter medical and psychosocial late effects of breast cancer and its treatment [50]. We presented an overview of the risks of developing health symptoms associated with each of the breast cancer treatment modalities up to five years after completing curative treatment [51]. Subsequently, we identified health symptoms that particularly have an impact on health-related quality of life and therefore are more pertinent to detect and manage than others [52]. Thus, follow-up could become more personalised by predicting which health symptoms may occur in patients; it could also become more effective, by targeting those health symptoms for which the biggest impact on the patient's life is expected. Symptoms that were particularly and significantly associated with a decrease in quality of life were musculoskeletal symptoms, fatigue, symptoms of the central nervous system, and gastro-intestinal symptoms [52]. Because over $50 \%$ of respondents experienced musculoskeletal symptoms, fatigue, and symptoms of the central nervous system [51], successful management of these symptoms would have a large effect on breast cancer survivorship. However, patients were currently not optimally involved in making follow-up decisions, nor was it optimally acknowledged they had preferences towards these decisions. Among these decisions were decisions about anti-hormonal therapy and the management of treatment-induced menopausal symptoms [31]. We suggest informing and involving patients in these decisions may lead to follow-up care that is adjusted to personal needs and values. 


\section{Towards personalised care: implications for clinical practise}

Several actions could be undertaken towards the provision of personalised breast cancer care. In order for a breast cancer patient to make an informed decision, she needs to be aware that there is a decision to be made, and she needs to receive the most up-to-date evidence-based information about the risks and benefits of each treatment option within this decision. Subsequently, the physician's advice and the patient's preferences are both incorporated in the final treatment decision [35, 36]. Thus, towards making good quality decisions based on both evidence and preferences, we need 1) sufficient evidence on the effects and outcomes of treatment options; 2) ways of properly communicating this evidence to patients; and 3) means to identify, express, and communicate patient needs and preferences.

First, an important facilitator in creating sufficient evidence about the effects and outcomes of treatment options may be the (further) application of Patient Reported Outcome Measures (PROMs). PROMs are important for generating evidence about the outcomes of specific treatment options [53]. Because of the relatively high breast cancer survival rates [54] and a growing interest in value-based healthcare [55], the effects of breast cancer treatment on the patients' quality of life become a more important rationale for treatment decisions [56]. Furthermore, by including preference-based quality of life measures, such as the EQ-5D, the way the general public values quality of life is included as well [57]. Thereby, the relevance of certain outcomes for society is included, and can be related with treatment costs. Based on these outcomes, we could steer towards care that leads to the best outcomes per money spent.

Second, towards properly communicating this evidence to patients, we believe information provision could be improved by the implementation of decision-aids and nomograms. Decisionaids aim to make decisions explicit to patients, to provide information about options and associated benefits and risks, and to help clarify congruence between decisions and personal values. In general, patients who use decision-aids feel more knowledgeable, better informed, know better what is important to them, have more accurate risk perceptions, and they are likely to have a more active role in decision making [58]. Because we identified that patients were currently not optimally involved in making decisions about follow-up [31], the decision aid which is developed by Klaassen et al. [59] could support women in making decisions about their aftercare schedule. Although use of this tool led to an increase in consultation time and thus costs, these costs were levelled out by the reduction of hospital costs, as significantly more patients chose for less intensive follow-up care [60]. For breast reconstruction options, several decision aids already have been developed [31, 61-65]. However, we believe decision aids should also be more explicit about uncertainties in evidence, so these can be communicated better to patients as well $[34,66]$. This is particularly important for decisions about chemotherapy timing. Neoadjuvant and adjuvant chemotherapy yield similar disease free and overall survival $[12,13]$; however, as current evidence is still preliminary [15, 16], decisions are made under uncertainty [34]. Consequently, caution is needed with framing this decision without oversimplifying the clinical situation [67]. Decision aids could provide more insight in difficult decisions like these. 
Nomograms depict a statistical prognostic model by using clinical variables to generate a probability of a clinical event for a given individual [68]. For instance, a nomogram might help in predicting the odds that breast conserving surgery will be feasible after neoadjuvant chemotherapy, based on the patients individual tumour characteristics. Vos et al. [69] describe a prediction model for cosmetic outcome after either breast conserving surgery or mastectomy, that could possibly provide this guidance. Furthermore, nomograms may be key in personalising follow-up care: a nomogram has already been developed specifically for the personalisation of recurrence surveillance. The "INFLUENCE nomogram" can calculate a prognosis for the risk of contralateral breast cancer [41], and based on this risk estimation, high and low risk patients can be identified, for which more and less intense surveillance frequencies can be used, respectively. Preventing overuse of surveillance is important from an ethical perspective, as literature described that surveillance appointments are associated with stress and anxiety [70]. However, as both breast cancer incidence and survival are high, with worldwide 2.1 million new diagnoses in 2018 [71] and five-year survival rates over $80 \%$ in North America and Europe [54], allocation of resources for follow-up becomes more pertinent from a health-economic perspective as well. We recommend that this nomogram should be extended with the findings from chapter 5 and $6[51,52]$, and consequently provides individualised risk information about physical and psychosocial effects at the same time. By that, patients will better understand the effects of treatment during treatment decision-making, and can understand how these effects may interfere with aspects in life they find important. Such a nomogram would provide a complete overview of what to expect after breast cancer, and is currently developed in the Primary Secondary Cancer Care Registry Project [72]. However, still more efforts need to be made to implement these nomograms. Rabin et al. [73] reported that, for 22 cancer prognostic tools they identified in their review, patient-involvement with these tools was not discussed and use in clinical practise was only limited. Salz et al. [74] reported that few of the identified prediction models for late effects were currently useful for risk-stratifying prevention, early detection or management of late effects. Towards implementation and validation, we believe platforms like Evidencio.com, an online public library for medical prediction models, could promote and facilitate the use of these existing nomograms [75].

Third, it is important to make explicit the patient's needs and preferences in these decision, and thus we need means to identify, express, and communicate these. Individual assessment of the patient's health status through PROMs could provide important information about the patient's individual needs $[53,76,77]$. PROMs comprise symptom scales about for instance physical impairments, psychosocial and psychosexual symptoms, and body image [6-8], enabling assessment of these aspects in order to screen or monitor patients' needs. Basch et al. [78] describes use of PROM symptom reporting during chemotherapy treatment had a positive effect on quality of life and resulted in less healthcare use. We believe similar application should be explored for breast cancer follow-up [43,50]. However, to fully capture the effect of breast cancer on the patient's life, domain measures about the patients' everyday lives, including finances, work, and family roles, may be also applicable here $[79,80]$. Yet, widespread standardised use of validated PROMs in daily practise by health systems is not yet common $[53,56]$, and the standardised assessment of outcomes is currently one of the major challenges for value-based healthcare [81]. Barriers of implementation for clinicians include negative beliefs about PROMs, 
time constraints, technological barriers, lack of reimbursement, and impact on the patient flow and clinical workflow, resources, and reimbursement systems [76, 82]. To further implement PROMs in everyday practise, we need new approaches, such as adoption of new data collection and analyses technologies [53,56], PROM scoring [56], assessment $[50,76]$, interpretation $[50,56$, $83]$ and presentation methods [ $53,56,83,84]$, and improvement of precision and responsiveness to reduce sample sizes through for instance item banking [85-87]. Yet, the challenge or solution may not be how to deploy PROM-tools, but rather how to create a system in which PROs are fundamental elements of the organisation of care [76]. This includes integration PRO-data with clinical data in the electronic health record [88], real-time presentation of quality of life scores during consultations [56], and direct comparison with the general population or 'patients-like-me' [89]. Last, guidelines should include recommendations on how to respond to divergent PROMscores $[50,83]$. By that, physicians are guided in applying the data in practice, and the focus of consultations will lie on the quality of life scores that are below average, becoming the pivot of for instance follow-up care.

Furthermore, shared decision-making (SDM) can help in making patient preferences explicit and in incorporating these in the final treatment decision $[35,36]$. However, the implementation of SDM in clinical practise has some challenges. First, a pitfall for SDM is the believe that physicians already engage in SDM or that an SDM-tool will 'do the work' [90], although evidence shows that implementation of SDM has not been optimal yet in clinical practise [36, 90]. Training health care providers and raising knowledge and awareness about SDM are important facilitators for implementing SDM $[35,36]$. Several interventions have been developed for training and educational purposes, and although it is better than no use of interventions, their effectiveness has not been robustly proven yet [91]. Second, some patient populations may seem reluctant to SDM, particularly the vulnerable patients, i.e. with lower health literacy [90]. Yet, these patients may be the ones most benefiting from SDM. This is where decision-support tools come in useful as well [36], which could be specifically tailored for these populations [90]. Furthermore, professional resistance may hamper (further) implementation. However, some wide-spread negative beliefs about SDM among physicians were disproved by Legare and Thompson-Leduc. For instance, there is no evidence that SDM takes up (too) much time, or will lead to increased health care costs [90]. Besides, it is becoming clear that not every patient wants to be maximally informed about his or her treatment or treatment options, but rather that the physician 'manages' the information [92]. For instance, De Rooij et al. [93] demonstrated that patients with an information seeking style felt more satisfied compared to patients with an information-avoiding style when they were informed by survivorship care plans. Thus, from an ethical perspective, promoting SDM remains important [36]. Last, what may complicate SDM for decisions about breast cancer, is that the decisions are interdependent to a certain degree. Therefore, SDM may not be restricted to one patient and one physician [90]. Involvement of other health professionals offers opportunities as well, for instance in providing patients an extra moment of deliberation [36]. However, this requires coordination, for example in the form of multidisciplinary team meetings. Here, PROMs can inform the members of the multidisciplinary team by providing a broader insight in the patients' health and wellbeing [77]. These multidisciplinary efforts are also important for immediate breast reconstruction, 
since the attendance of a plastic surgeon at the multidisciplinary team meetings was significantly associated with higher rates of immediate breast reconstruction [27].

Decision-making process for breast reconstruction as 'best practice'

We believe that the decision-making process for breast reconstruction could be a leading example for other decision-making processes for breast cancer treatment and follow-up care. Again, we base this on the availability of 1 ) sufficient evidence on the effects and outcomes of treatment options; 2 ) ways of properly communicating this evidence to patients; and 3) means to identify, express, and communicate patient needs and preferences. First, evidence-based tradeoffs can be made, as we increasingly understand the effects of breast reconstruction on quality of life [94-98], including type of reconstruction [96], possible surgical complications [99-102], and the effects of other treatment modalities such as radiotherapy on outcomes of implantbased reconstructions $[103,104]$. Besides, this evidence was recently summarized in a guideline specifically for breast reconstruction [25]. Second, although we concluded information provision was not entirely optimal [30] and several gaps in information provision about breast reconstruction were identified in our scoping review [31], multiple decision-support tools have been developed towards improving breast reconstruction information provision as well [31, 61-65]. Third, four-fifth of patients reported that the final treatment decision about (immediate) breast reconstruction was in line with her preferences or clinical characteristics [30], suggesting this decision-making process already leads to personalised treatment decisions. Besides, we see several opportunities for further refinement of decision-making concerning breast reconstruction. For example, we demonstrated that breast preservation and reconstruction options each had their own favourable outcomes based on quality of life domain scores [94], that provide more specific information than a single overarching quality of life score. Furthermore, as these outcomes may be affected by individual patient and treatment characteristics or indications [25], it would be helpful to predict which of these characteristics affect the outcomes of an individual patient and to what degree. A risk prediction model, presented in a nomogram, could provide patients and physicians with personalised advice on what to expect per type of breast reconstruction in terms of complications and quality of life. By extending a currently existing decision-aid for breast reconstruction with a nomogram application, it has the potential to combine patient preferences, clinical indications, and expected outcomes to optimally inform patients about the possible final result of each procedure. A side note, however, is that not all decisions may be as 'easy to frame' as decisions about breast reconstruction. Whereas decisions about breast reconstruction are rather elective in nature, decisions about for example the timing of chemotherapy are influenced by evidence that is still preliminary $[15,16]$. The possible consequences of such a decision may therefore be of another magnitude than decisions about for instance breast reconstruction.

\section{Limitations of this thesis}

Questionnaires administrated to patients and physicians were the main method of data collection in this thesis. However, use of questionnaires may be subject to several forms of bias, including recall bias, selection bias (as some patients were excluded from participation based on their characteristics), response bias (some patients responded while others did not), bias by indication (not all patients are eligible for each treatment option), and interpretation bias (patients might 
have interpreted questions in an individual way). For all studies in this thesis based on patientreported outcomes, patients reported their experiences with information provision and SDM in retrospect, possibly creating recall bias. To overcome this problem, we tried to balance patient experiences with chemotherapy decisions with beliefs and indications about chemotherapy timing as reported by physicians, creating a diptych of evidence. For decisions about immediate breast reconstruction in the second part, we can correlate our findings with leading beliefs and indications from physicians as well [105]. Alongside recall bias, the effect of cognitive dissonance may have resulted in current opinions to be more in line with the decisions that were initially taken at time of treatment [106]. In the studies that reported on quality of life, we expect recall bias was less present, as patients reported their current quality of life. However, as the result from response shift, patient might value their health-related quality of life differently now than they did before cancer diagnosis [107]. Regarding selection bias, patients who could not read or write in Dutch were excluded upfront from all studies, providing a more one-sided reflection of society than it in reality is. This limits the generalizability of our findings, as we expect rather different outcomes for these patients. We believe information provision and shared decision-making in more vulnerable patients, i.e. with low health literacy, should receive attention as a separate research topic. We expect similar effects caused by response bias. For instance, race, ethnicity, and annual household income were found to be associated with nonresponse to a survey about breast reconstruction [108]. Response bias may lead to an underestimation of quality of life effects, even in studies with relatively high participation rates, as participation was associated with factors as high socioeconomic status and having no comorbidities [109]. In the majority of our patient-reported studies, a response rate of about $50 \%$ was reached. Because nearly all our patient-reported data was merged with clinical data from the Netherlands Cancer Registry, we at least had insight in the patient, tumour and treatment characteristics of both responders and non-responders. However, even in studies were outcomes were corrected for preoperative characteristics, still slightly worse outcomes were found for late responders versus 'fast' responders [110]. We need more insight in the effects of response bias for PROMs, rather than (only) minimizing non-response rates [108]. Also, bias by indication was a challenge for quality of life in patients treated with different surgical interventions, as included for Chapter 3. A propensity-score weighting for multiple treatments was calculated to adjust for covariates that predict receiving either one of the four surgical treatment options. Finally, to minimise interpretation bias, the majority of our questionnaires was assessed on readability and comprehensibility by a panel of (former) breast cancer patients. These panels also payed attention to how questions were framed, as framing may steer patients towards certain answers [111].

Furthermore, the use of outcome measures such as patient preferences, needs, and experiences with information provision and shared decision-making has some difficulties, as they tend to consist of multiple facets. For instance, the inclusion of the concept 'preferences' is complicated, as they are the result of a complex interplay between intuitive and deliberative mental processes, that may or may not remain stable over time [67]. Furthermore, Charles et al. [112] stated that the concept of SDM is still open to different interpretations, thus measuring it remains problematic. PROMs are based on conceptual models that provide the rationale for and specification of the PROs of interest [113]. We tried to select validated PROMs to overcome issues as construct validity 
and content validity. However, for information provision and SDM specifically about chemotherapy timing and breast reconstruction, these were not available. Thus, one might sense these concepts are difficult to capture in a complete and uniform way.

\section{Future research}

We have demonstrated that treatment variation can result from variation in information provision, shared decision-making, and patient preferences, but also from physicians' leading beliefs and indications, for three aspects of breast cancer treatment and follow-up. However, we believe these are just examples of how to indicate variation and we suggest other aspects of cancer care and breast cancer care should be subjected to analyses similar as in this thesis.

To optimise decision-making through information provision and shared decision-making, and to understand variation, we need more evidence in terms of patient-reported outcomes, complementary to already available clinical evidence, for several reasons. First, in terms of patientreported outcomes research, to understand what happens to patients during and after breast cancer treatment on both the short and long run. By the further development of nomograms, we can also use these data in prospect, to predict and anticipate on what patients will go through when making a treatment decision. The further development of these models should include proper validation, risk presentation in an understandable way, collection of longitudinal PROM data, and clever use of existing data. But also in terms of detection of side effects of treatment and of health symptoms during follow-up, further development of PROMs is important. Up to five years after diagnosis, almost all breast cancer patients experienced health symptoms. For the symptoms that are most prevalent and have a large impact of health-related quality of life, we need effective interventions to manage these symptoms. PROMs are an important outcome measure in research into these interventions as well.

With PROM evidence, we will be able to better inform patients prior to treatment decision-making about treatment effects. We suggest it should be specifically stated in guidelines when a treatment decision should be discussed, thus highlighting the need of informing patients about their options [36]. Currently, we move from guidelines in text form to guidelines in the format of decision-trees, as for instance developed in the OncoGuide project [114]. We expect that highlighting these decision 'moments' will perfectly blend in the format of decision-trees. Also, at each 'decision knot', we can tie suggestions for relevant decision-aids and nomograms. And, when we include cut-offs or guidance for the interpretation of PROM scores [83], the patients' needs can become the pivot of follow-up consultations which is specifically relevant for follow-up care.

Furthermore, we should identify best practices for information provision and SDM, so that further implementation of both can take place. Besides, treatment decision-making in terms of investigating trade-offs and thresholds, but also its effectiveness in terms of self-efficacy and decisional regret, are fields of research in itself. We do believe decision-analysis can help in improving the current decision-making processes. An example is described in the study by Sun et al. [115], in which the elements of decision-making for breast reconstruction were set forth. 
Last, the studies presented in this thesis explored variation in breast cancer care from a patients perspective, therefore focussing on aspects of variation such as information provision and SDM, that are in particular important for individual patients. However, in determining causes of variation, relevant variables include patient- and clinical characteristics and physician, hospital, and organisational factors as well. Specifically for breast cancer care in the Netherlands, the influence of some of these factors has been studied. First, a study including all surgically treated stage III breast cancer patients in the Netherlands revealed that organisational factors such as use of breast MRI and hospital participation in neoadjuvant clinical studies were significant independent predictors of neoadjuvant chemotherapy use (all $P<0.001$ ). In addition, patientrelated factors as age $<50$ years, and disease-related factors as large tumour size, advanced nodal disease, and negative hormone receptor status were also independent predictors of neoadjuvant chemotherapy use [22]. It would be of interest to look into this for stage II patients as well. Second, immediate breast reconstruction was more often used in younger patients, multifocal tumours, smaller tumours, tumours with a lower grade, absence of lymph node involvement, ductal carcinomas, or hormone-receptor positive/HER2-positive tumours. However, even after correction for these factors, considerable variation remained, suggesting hospital and organizational factors may be of importance as well. Therefore, organisational factors were studied for immediate breast reconstruction as well. Hospital type, number of plastic surgeons available at the hospital, and attendance of a plastic surgeon at the multidisciplinary team meeting increased the rate of immediate breast reconstruction [27]. Furthermore, following a survey among surgical oncologists and plastic surgeons in the Netherlands about indications and contraindications for immediate breast reconstruction, it became evident that physicians differed in their opinions about these contra-indications. For example, plastic surgeons more frequently reported patientrelated risk factors of wound healing problems as contra-indication, while surgical oncologists reported more oncology-related contra-indications [105]. Evidence about surgical complications and reconstruction failure could help in creating consensus about contra-indications. Third, follow-up practice for breast cancer in the Netherlands was screened by the National Health Care Institute (Zorginstituut Nederland) to compose a plan of improvement towards appropriate care provision. They concluded that breast cancer follow-up could be improved by implementing risk-based screening for recurrent breast cancer, and by investing in proper information provision and SDM about effects of breast cancer and its treatment [47], suggesting organisational factors are affecting the provision of follow-up care as well. However, as these improvements have not yet been implemented in practice, the (health economic) evaluation of the adaptation of followup management would be a highly relevant subject for future research.

\section{Final words on this thesis}

This thesis demonstrates that treatment variation can result from information provision, shared decision-making, patient preferences, and physicians leading beliefs and indications in three aspects of breast cancer treatment and follow-up. For all three aspects, we have suggested improvements, and therefore expect the following trends for treatment personalisation and variation in the future. We believe decisions about the timing of chemotherapy will become more personalised when sufficient evidence about the risks and benefits of neoadjuvant chemotherapy will become available [116]. This may subsequently lead to an increase of the application of 
neoadjuvant chemotherapy, and likely as well and increase in breast conserving surgery. The past years, we already saw an increase in use of neoadjuvant chemotherapy and in patients receiving breast conserving surgery after neoadjuvant chemotherapy $[117,118]$. Moreover, in the trend of de-escalating treatment [50], breast conserving surgery has replaced mastectomy as the preferred surgical option for treating early-stage breast cancer $[116,119]$. This will affect the percentage of the breast cancer population opting for mastectomy with or without (immediate) breast reconstruction [119]. Within the group of mastectomy patients, we detected a small need for improvement of information provision. Yet, as information increased the odds of breast reconstruction by 14 times, a small increase in application of breast reconstruction is expected within the group of patients indicated or opting for mastectomy. Last, the necessity of personalized follow-up will become more pertinent, leading to ongoing efforts to align care with actual patient needs. Personalised follow-up care is not only relevant from a patient-centred perspective. With the increasing breast cancer prevalence, the access to follow-up care for the entire breast cancer population becomes under pressure. Therefore, personalisation of follow-up care becomes a necessity if we want to preserve equal and cost-effective access of care for every breast cancer patient in need of follow-up.

However, what is most important for all three examples, is that not the reduction of variation is the ultimate goal, but the improvement of quality of care through personalization of care. We more and more shift towards context-based evidence and practise-based medicine rather than strict evidence-based medicine [120], in part due to population-based data and quality registries as the Netherlands Cancer Registry and the NABON Breast Cancer Audit. However, that does not mean we should provide patients with every care they think they need, as if they can 'shop' for healthcare. A pitfall for patient-centred care is that care should be tailored towards the patient's needs, and not towards what they want or they believe they need [121]. Dare to tell the patient that not everything that is available, is necessary or beneficial [122]: if we align evidence of treatment effectiveness and patient preferences, we expect to reach healthcare that is of most value for individual patients. By doing this, patients are not only the subject of care, but also deliver input for care. We should extend this paradigm by including patients in research design and agenda setting, as we believe they can indicate what is needed towards patient-centred care. 


\section{References}

1. Netherlands Comprehensive Cancer Organisation (IKNL). About the registration 2018 [Available from: https://www.cijfersoverkanker.nl/about-the-registration-37.html.

2. van Bommel ACM, Spronk PER, Vrancken Peeters MJTFD, Jager A, Lobbes M, Maduro JH, et al. Clinical auditing as an instrument for quality improvement in breast cancer care in the Netherlands: The national NABON Breast Cancer Audit. Journal of surgical oncology. 2017;115(3):243-9.

3. van de Poll-Franse LV, Horevoorts N, Eenbergen M, Denollet J, Roukema JA, Aaronson NK, et al. The Patient Reported Outcomes Following Initial treatment and Long term Evaluation of Survivorship registry: Scope, rationale and design of an infrastructure for the study of physical and psychosocial outcomes in cancer survivorship cohorts. European journal of cancer (Oxford, England : 1990). 2011;47(14):2188-94.

4. Trask PC, Tellefsen C, Espindle D, Getter C, Hsu MA. Psychometric Validation of the Cancer Therapy Satisfaction Questionnaire. Value in Health. 2008;11(4):669-79.

5. Herdman M, Gudex C, Lloyd A, Janssen MF, Kind P, Parkin D, et al. Development and preliminary testing of the new five-level version of EQ-5D (EQ-5D-5L) Qual Life Res. 2011;20:1727-36.

6. Aaronson NK, Ahmedzai S, Bergman B, Bullinger M, Cull A, Duez NJ, et al. The European Organisation for Research and Treatment of Cancer QLQ-C30: A quality-of-life instrument for use in international clinical trials in oncology. J Natl Cancer Inst. 1993;85:365-76.

7. Sprangers MAG, Groenvold M, Arraras JI, Franklin J, te Velde A, Muller M, et al. The European Organisation for Research and Treatment of Cancer: Breast Cancer Specific Quality of Life Questionnaire Module: First results from a three-country field study. J Clin Oncol. 1996;14:2756-68.

8. Cano SJ, Klassen AF, Scott AM, Pusic AL. A Closer Look at the BREAST-Q. Clin Plastic Surg. 2013;40:287-96.

9. Pusic AL, Klassen AF, Scott AM, Klok JA, Cordeiro PG, Cano SJ. Development of a new patient-reported outcome measure for breast surgery: the BREAST-Q. Plast Reconstr Surg. 2009;124(2):345-53.

10. Mieog JSD, Hage JA van der, Velde $\mathrm{CJH}$ van de. Neoadjuvant chemotherapy for operable breast cancer. $\mathrm{Br}$ J Surg. 2007(94):1189-200.

11. Barranger E, Antomarchi J, Chamorey E, Cavrot C, Flipo B, Follana P, et al. Effect of Neoadjuvant Chemotherapy on the Surgical Treatment of Patients With Locally Advanced Breast Cancer Requiring Initial Mastectomy. Clinical breast cancer. 2015;15(5):e231-5.

12. Rastogi P, Anderson SJ, Bear HD, Geyer CE KM, Robidoux A, Margolese RG, et al. Preoperative chemotherapy: updates of National Surgical Adjuvant Breast and Bowel Project Protocols B-18 and B-27. J Clin Oncol. 2008;26(5):778-85.

13. Mauri D, Pavlidis N, loannidis JP. Neoadjuvant versus adjuvant systemic treatment in breast cancer: a metaanalysis. J Natl Cancer Inst. 2005;97(3):188-94.

14. Mougalian SS, Hernandez M, Lei X, Lynch S, Kuerer HM, Symmans WF, et al. Ten-Year Outcomes of Patients With Breast Cancer With Cytologically Confirmed Axillary Lymph Node Metastases and Pathologic Complete Response After Primary Systemic Chemotherapy. JAMA Oncology. 2016;2(4):508-16.

15. von Minckwitz G, Untch M, Nüesch E, Loibl S, Kaufmann M, Kümmel S, et al. Impact of treatment characteristics on response of different breast cancer phenotypes: pooled analysis of the German neoadjuvant chemotherapy trials. Breast Cancer Res Treat. 2011;125(1):145-56.

16. Cortazar P, Zhang L, Untch M, Mehta K, Costantino JP, Wolmark N, et al. Pathological complete response and long-term clinical benefit in breast cancer: the CTNeoBC pooled analysis. Lancet. 2014;384(9938):164-72.

17. Netherlands Comprehensive Cancer Organisation (IKNL). National guideline on Breast Cancer. Netherlands Comprehensive Cancer Organisation (IKNL),,; 2012.

18. Holmes D, Colfry A, Czerniecki B, Dickson-Witmer D, Francisco Espinel C, Feldman E, et al. Performance and Practice Guideline for the Use of Neoadjuvant Systemic Therapy in the Management of Breast Cancer. Ann Surg Oncol. 2015;22(10):3184-90.

19. Cardoso F, Costa A, Norton L, Senkus E, Aapro M, André F, et al. ESO-ESMO 2nd international consensus guidelines for advanced breast cancer (ABC2). The Breast. 2014;23(5):489-502. 
20. Mougalian SS, Soulos PR, Killelea BK, Lannin DR, Abu-Khalaf MM, DiGiovanna MP, et al. Use of neoadjuvant chemotherapy for patients with stage I to III breast cancer in the United States. Cancer. 2015;121(15):254452.

21. Dutch Institute for Clinical Auditing. Jaarrapportage 2013. NABON Breast Cancer Audit; 2014.

22. Spronk PER, van Bommel ACM, Vrancken Peeters MTFD, Siesling S, Smorenburg CH. Variation in the use of neoadjuvant chemotherapy for stage III breast cancer: results of the Dutch nationwide breast cancer registry NBCA (Nabon Breast Cancer Audit). The Breast. 2017;36:34-8.

23. Cordeiro PG. Breast Reconstruction after Surgery for Breast Cancer. New England Journal of Medicine. 2008(359):1590-601.

24. Senkus E, Kyriakides S, Ohno S, Penault-Llorca F, Poortmans P, Rutgers E, et al. Primary breast cancer: ESMO Clinical Practice Guidelines for diagnosis, treatment and follow-up. Annals of Oncology. 2015;26:8-30.

25. Mureau MAM, Nederlandse Vereniging voor Plastische Chirurgie (NVPC). Dutch Breast Reconstruction Guideline. Journal of Plastic Reconstructive and Aesthetic Surgery 2018:290-304.

26. van Bommel ACM, Mureau MAM, Schreuder K, van Dalen T, Vrancken Peeters MTFD, Schrieks M, et al. Large variation between hospitals in immediate breast reconstruction rates after mastectomy for breast cancer in the Netherlands. Journal of Plastic, Reconstructive \& Aesthetic Surgery. 2017;70(2):215-21.

27. Schreuder K, Van Bommel ACM, De Ligt KM, Maduro JH, Vrancken Peeters MTFD, Mureau MAM, et al. Hospital organizational factors affect the use of immediate breast reconstruction after mastectomy for breast cancer in the Netherlands. Breast (Edinburgh, Scotland). 2017;34:96-102.

28. Runowicz CD, Leach CR, Henry NL, Henry KS, Mackey HT, Cowens-Alvarado RL, et al. American Cancer Society/American Society of Clinical Oncology Breast Cancer Survivorship Care Guideline. Journal of Clinical Oncology. 2016;34(6):611-35.

29. Grunfeld E, Dhesy-Thind S, Levine M. Clinical practice guidelines for the care and treatment of breast cancer: follow-up after treatment for breast cancer (summary of the 2005 update). Canadian Medical Association Journal. 2005;172(10).

30. de Ligt KM, van Bommel ACM, Schreuder K, Maduro JH, Vrancken Peeters MTFD, Mureau MAM, et al. The effect of being informed on receiving immediate breast reconstruction in breast cancer patients. European journal of surgical oncology : the journal of the European Society of Surgical Oncology and the British Association of Surgical Oncology. 2018;44(5):717-24.

31. de Ligt KM, van Egdom LSE, Koppert LB, Siesling S, van Til JA. Opportunities for personalised follow-up care among patients with breast cancer: a scoping review to identify preference sensitive decisions European Journal of Cancer Care. 2019;28(3):e13092.

32. de Ligt KM, Spronk PER, van Bommel ACM, Vrancken Peeters MTFD, Siesling S, Smorenburg CH. Patients' experiences with decisions on timing ofchemotherapy for breast cancer. The Breast. 2018;37(99-106).

33. Spronk PER, de Ligt KM, van Bommel ACM, Siesling S, Smorenburg CH, MTFD; VP, et al. Current decisions on neoadjuvant chemotherapy for early breast cancer: experts' experiences in the Netherlands. Patient Educ Couns. 2018;101(12):2111-5.

34. Shelton RC, Brotzman LE, Crookes DM, Roblesa P, Neugut Al. Decision-making under clinical uncertainty: An in-depth examination of provider perspectives on adjuvant chemotherapy for stage II colon cancer. Patient Educ Couns. 2019;102:284-90.

35. Légaré F, Witteman HO. Shared Decision Making: Examining Key Elements And Barriers To Adoption Into Routine Clinical Practice. Health Affairs. 2013;32(2):276-84.

36. Stiggelbout AM, Pieterse AH, De Haes JCJM. Shared decision making: Concepts, evidence, and practice. Patient Education and Counseling. 2015;98:1172-9.

37. Flitcroft K, Brennan M, Spillane A. Decisional regret and choice of breast reconstruction following mastectomy for breast cancer: A systematic review. Psychooncology. 2018;27:1110-20.

38. Kunneman M, Engelhardt EG, Ten Hove FL, Marijnen CA, Portielje JE, Smets EM, et al. Deciding about (neo-) adjuvant rectal and breast cancer treatment: Missed opportunities for shared decision making. Acta Oncol. 2016;55(2):134-9.

39. Porter ME, Teisberg EO. How Physicians Can Change the Future of Health Care. JAMA. 2007;297(10):110311. 
40. Porter ME. Value-Based Health Care Delivery. Annals of Surgery. 2008;248(4).

41. Witteveen A, Vliegen IMH, Sonke GS, Klaase JM, IJzerman MJ, Siesling S. Personalisation of breast cancer follow-up: a time-dependent prognostic nomogram for the estimation of annual risk of locoregional recurrence in early breast cancer patients. Breast Cancer Research and Treatment. 2015;152(3):627-36.

42. de Oliveira RAA, da Conceição VM, Araujo JS, Zago MMF. Concept analysis of cancer survivorship and contributions to oncological nursing. International Journal of Nursing Practise. 2018;24(e12608).

43. Fallowfield L, Jenkins V. Psychosocial/Survivorship Issues in Breast Cancer: Are We Doing Better? J Natl Cancer Inst. 2015;107(1):dju335.

44. DH Macmillan Cancer Support, NHS Improvement. National Cancer Survivorship Initiative: Vision. 2010.

45. Donnelly P, Hiller L, Bathers S, Bowden S, Coleman R. Questioning specialists' attitudes to breast cancer follow-up in primary care. Annals of Oncology. 2007;18:1467-76.

46. Montgomery DA, Krupa K, Cooke TG. Alternative methods of follow up in breast cancer: a systematic review of the literature. British Journal of Cancer. 2007;96:1625-32.

47. Zorginstituut Nederland. Verbetersignalement Zinnige nacontrole bij vrouwen behandeld voor borstkanker. Diemen: Zorginstituut Nederland; 2016.

48. Kwast ABG, Drossaert CHC, Siesling S. Breast cancer follow-up: from the perspective of health professionals and patients. European Journal of Cancer Care. 2013;22:754-64.

49. Rosselli Del Turco M, Palli D, Cariddi A, Ciatto S, Pacini P, Distante V. Intensive diagnostic follow-up after treatment of primary breast cancer. A randomized trial. National Research Council Project on Breast Cancer follow-up. JAMA. 1994;271(20):1593-7.

50. Cardoso F, Harbeck N, Barrios CH, Bergh J, Cortés J, El Saghir N, et al. Research needs in breast cancer. Annals of Oncology. 2017;28:208-17.

51. de Ligt KM, Heins M, Verloop J, Smorenburg CH, Korevaar JC, Siesling S. Patient-reported health problems and healthcare use after treatment for early-stage breast cancer. The Breast. 2019;4(46):4-11.

52. de Ligt KM, Heins M, Verloop J, Ezendam NPM, Smorenburg CH, Korevaar JC, et al. The impact of health symptoms on health-related quality of life in earlystage breast cancer survivors. Submitted for publication at Cancer, 2019.

53. Black N. Patient reported outcome measures could help transform healthcare. British Medical Journal. 2013;346:f167.

54. Coleman MP, Quaresma M, Berrino F, Lutz JM, De Angelis R, Capocaccia R, et al. Cancer survival in five continents: a worldwide population-based study (CONCORD). Lancet Oncology. 2008;9:730-56.

55. Porter ME, Ishrak O. The State of Adoption in Value-Based Health Care. 2015.

56. Tevis SE, James TA, Kuerer HM, Pusic AL, Yao KA, Merlino J, et al. Patient-Reported Outcomes for Breast Cancer. Annals of Surgical Oncology. 2018;25:2839-45.

57. Gray AM, Clarke PM, Wolstenholme JL, Wordsworth S. Chapter 5: Measuring, valuing, and analysing health outcomes. Applied Methods of Cost-effectiveness Analysis in Health Care. Handbooks in Health Economic Evaluation Series. Oxford: Oxford University Press; 2011.

58. Stacey D, Légaré F, Lewis K, Barry MJ, Bennett CL, Eden KB, et al. Decision aids for people facing health treatment or screening decisions. Cochrane Database Syst Rev 2017;4(CD001431).

59. Klaassen LA, Dirksen CD, Boersma LJ, Hoving C. Developing an aftercare decision aid; assessing health professionals' and patients' preferences. Eur J Cancer Care (Engl). 2018;27(2).

60. Klaassen LA, Dirksen CD, Boersma LJ, Hoving C, On behalf of the B-beslist!-group. A novel patient decision aid for aftercare in breast cancer patients: A promising tool to reduce costs by individualizing aftercare. The Breast. 2018;41:144-50.

61. Heller L, Parker PA, Youssef A, Miller MJ. Interactive digital education aid in breast reconstruction. Plast Reconstr Surg. 2008;122(3):717-24.

62. Sherman KA, Shaw LK, Winch CJ, Harcourt D, Boyages J, Cameron LD, et al. Reducing Decisional Conflict and Enhancing Satisfaction with Information among Women Considering Breast Reconstruction following Mastectomy: Results from the BRECONDA Randomized Controlled Trial. Plast Reconstr Surg. 2016;138(4):592e-602e. 
63. Temple-Oberle C, Ayeni O, Webb C, Bettger-Hahn M, Ayeni O, Mychailyshyn N. Shared decision-making: applying a person-centered approach to tailored breast reconstruction information provides high satisfaction across a variety of breast reconstruction options. Journal of surgical oncology. 2014;110(7):796-800.

64. Causarano N, Platt J, Baxter NN, Bagher S, Jones JM, Metcalfe KA, et al. Pre-consultation educational group intervention to improve shared decision-making for postmastectomy breast reconstruction: a pilot randomized controlled trial. Supportive care in cancer : official journal of the Multinational Association of Supportive Care in Cancer. 2015;23(5):1365-75.

65. ter Stege JA, Woerdeman LAE, Hahn DEE, Oldenburg HSA, van Huizum MA, Witkamp AJ, et al. Impact of a web-based decision aid for women considering breast reconstruction: study protocol for a multicenter randomized controlled trial. Psycho-Oncology. 2016;25(S3):3-195.

66. Politi MC, Lewis CL, Frosch DL. Supporting Shared Decisions When Clinical Evidence Is Low. Medical Care Research and Review. 2013;70(1):113S-28S.

67. Katz SJ, Hawley S. The Value of Sharing Treatment Decision Making With Patients Expecting Too Much? JAMA. 2013;310(15):1559-60.

68. Balachandran VP, Gonen M, Smith JJ, DeMatteo RP. Nomograms in Oncology - More than Meets the Eye. Lancet Oncol. 2015;16(4):e173-e80.

69. Vos E, Koppert L, van Lankeren W, Verhoef C, Koerkamp BG, Hunink M. A preliminary prediction model for potentially guiding patient choices between breast conserving surgery and mastectomy in early breast cancer patients; a Dutch experience. Qual Life Res. 2018;27(2):545-53.

70. Brandzel S, Rosenberg DE, Johnson D, Bush M, Kerlikowske K, Onega T, et al. Women's experiences and preferences regarding breast imaging after completing breast cancer treatment. Patient preference and adherence. 2017;11:199-204.

71. Bray F, Ferlay J, Soerjomataram I, Siegel RL, Torre LA, Jemal A. Global Cancer Statistics 2018: GLOBOCAN Estimates of Incidence and Mortality Worldwide for 36 Cancers in 185 Countries. CA: A Cancer Journal for Clinicians. 2018;0:1-31.

72. Heins M, Verloop J, De Ligt KM, Siesling S, Korevaar J. Primary Secondary Cancer Care Registry (PSCCR): Following breast cancer patients from their first complaints up to 15 years after diagnosis. European Journal of Cancer. 2018;92(Supplement 3):S39-S40.

73. Rabin BA, Gaglio B, Sanders T, Nekhlyudov L, Dearing JW, Bull S, et al. Predicting cancer prognosis using interactive online tools: a systematic review and implications for cancer care providers. Cancer epidemiology, biomarkers \& prevention : a publication of the American Association for Cancer Research, cosponsored by the American Society of Preventive Oncology. 2013;22(10):1645-56.

74. Salz T, Baxi SS, Raghunathan N, Onstad EE, Freedman AN, Moskowitz CS, et al. Are we ready to predict late effects? A systematic review of clinically useful prediction models. European Journal of Cancer. 2015;51:75866.

75. Evidencio [web page]. [V2.16:[Available from: https://www.evidencio.com.

76. Donaldson MS. Taking PROs and patient-centered care seriously: incremental and disruptive ideas for incorporating PROs in oncology practice. Qual Life Res. 2008;17:1323-30.

77. Greenhalgh J. The applications of PROs in clinical practice: what are they, do they work, and why? Qual Life Res. 2009;18:115-23.

78. Basch E, Deal AM, Kris MG, Scher HI, Hudis CA, Sabbatini P, et al. Symptom Monitoring With PatientReported Outcomes During Routine Cancer Treatment: A Randomized Controlled Trial. Journal of Clinical Oncology. 2016;34(6).

79. Catt S, Starkings R, Shilling V, Fallowfield L. Patient-reported outcome measures of the impact of cancer on patients' everyday lives: a systematic review. Journal of Cancer Survivorship. 2017;11:211-32.

80. Shilling V, Starkings R, Jenkins V, Cella D, Fallowfield L. Development and validation of the patient roles and responsibilities scale in cancer patients. Quality of Life Research. 2018;27:2923-34.

81. Ong WL, Schouwenburg MG, van Bommel ACM, Stowell C, Allison KH, Benn KE, et al. A Standard Set of ValueBased Patient-Centered Outcomes for Breast Cancer: The International Consortium for Health Outcomes Measurement (ICHOM) Initiative. JAMA Oncology. 2017;3(5):677-85.

82. Basch E. Patient-Reported Outcomes - Harnessing Patients' Voices to Improve Clinical Care. N Engl J Med. 2017;376(2):105-8. 
83. Snyder CF, Aaronson NK, Choucair AK, Elliott TE, Greenhalgh J, MY H, et al. Implementing patient-reported outcomes assessment in clinical practice: a review of the options and considerations. Qual Life Res. 2012;21:1305-14.

84. Snyder C, Smith K, Holzner B, Rivera YM, Bantug E, Brundage M, et al. Making a picture worth a thousand numbers: recommendations for graphically displaying patient-reported outcomes data. Quality of Life Research. 2019;28:245-356.

85. Fries JF, Krishnan E, Rose M, Lingala B, Bruce B. Improved responsiveness and reduced sample size requirements of PROMIS physical function scales with item response theory. Arthritis Res Ther. 2011;13(5):R147.

86. Haverman L, Grootenhuis MA, Raat H, van Rossum MAJ, van Dulmen-den Broeder E, Hoppenbrouwers $\mathrm{K}$, et al. Dutch-Flemish translation of nine pediatric item banks from the Patient-Reported Outcomes Measurement Information System (PROMIS) . Qual Life Res. 2016;25:761-5.

87. Kulis D, Bottomley A, Whittaker C, van de Poll-Franse LV, Darlington A, Holzner B, et al. The Use of The Eortc Item Library To Supplement Eortc Quality of Life Instruments (PRM250). Value in Health. 2017;20:A399-A811.

88. Basch E, Snyder C. Overcoming barriers to integrating patient reported outcomes in clinical practice and electronic health records. Annals of Oncology. 2017;28(10):2332-3.

89. Oerlemans S, Arts LP, Horevoorts NJ, van de Poll-Franse LV. "Am I normal?" The Wishes of Patients With Lymphoma to Compare Their Patient-Reported Outcomes With Those of Their Peers. J Med Internet Res. 2017;19(8):e288.

90. Légaré F, Thompson-Leduc P. Twelve myths about shared decision making. Patient Educ Couns. 2014;96:2816.

91. Légaré F, Stacey D, Turcotte S, Cossi MJ, Kryworuchko J, GrahamID, et al. Interventions for improving the adoption of shared decision making by healthcare professionals. Cochrane Database Syst Rev. 2014;5:CD006732.

92. Atherton K, Young B, Kalakonda N, Salmon P. Perspectives of patients with haematological cancer on how clinicians meet their information needs: "Managing" information versus "giving" it. Psycho-Oncology. 2018;27:1719-26.

93. de Rooij BH, Ezendam NPM, Vos MC, Pijnenborg JMA, Boll D, Kruitwagen RFPM, et al. Patients' information coping styles influence the benefit of a survivorship care plan in the ROGY Care Trial: New insights for tailored delivery. Cancer. 2019;125(5):788-97.

94. Kouwenberg CAE, de Ligt KM, Kranenburg LW, Rakhorst H, Leeuw D, Siesling S, et al. Long-term healthrelated quality of life after four common surgical treatment options for breast cancer and the effect of complications - a retrospective patient-reported survey among 1871 patients. Submitted for publication at Journal of Plastic and Reconstructive Surgery. 2019.

95. Lagendijk M, van Egdom LSE, Richel C, van Leeuwen N, Verhoef C, Lingsma HF, et al. Patient reported outcome measures in breast cancer patients. European Journal of Surgical Oncology. 2018;44:963-8.

96. Santosa KB, Qi J, Kim HM, Hamill JB, Wilkins EG, Pusic AL. Long-term Patient-Reported Outcomes in Postmastectomy Breast Reconstruction. JAMA surgery. 2018;153(10):891-9.

97. Eltahir Y, Werners LLCH, Dreise MM, IA ZvE, Jansen L, Werker PMN, et al. Quality-of-life outcomes between mastectomy alone and breast reconstruction: comparison of patient-reported BREAST-Q and other healthrelated quality-of-life measures. Plastic and reconstructive surgery. 2013;132(2):201e-9e.

98. Kim MK, Kim T, Moon HG, Jin US, Kim K, Kim J, et al. Effect of cosmetic outcome on quality of life after breast cancer surgery. European journal of surgical oncology : the journal of the European Society of Surgical Oncology and the British Association of Surgical Oncology. 2014;41(3):426-32.

99. Gopie JP, Timman R, Hilhorst MT, Hofer SOP, Mureau MAM, Tibben A. The short-term psychological impact of complications after breast reconstruction. Psychooncology. 2013;22:290-8.

100. Higgins KS, Gillis J, Williams JG, LeBlanc M, Bezuhly M, Chorney JM. Women's Experiences With Flap Failure After Autologous Breast Reconstruction: A Qualitative Analysis. Ann Plas Surg. 2017;78(5):521-5.

101. Timman R, Gopie JP, Brinkman JN, Kleijne A, Seynaeve C, Menke-Pluymers MBE, et al. Most women recover from psychological distress after postoperative complications following implant or DIEP flap breast reconstruction: A prospective long-term follow-up study. PloS one. 2017;12(3):e0174455. 
102. Lu SM, Nelson JA, Fischer JP, Fosnot J, Goldstein J, Selber JC, et al. The impact of complications on function, health, and satisfaction following abdominally based autologous breast reconstruction: A prospective evaluation. J Plast Reconstr Aes 2014;67(5):682-92.

103. Magill LJ, Robertson FP, Jell G, Mosahebi A, Keshtgar M. Determining the outcomes of post-mastectomy radiation therapy delivered to the definitive implant in patients undergoing one- and two-stage implantbased breast reconstruction: A systematic review and meta-analysis. JPRAS. 2017;70:1329-35.

104. Jagsi R, Li Y, Morrow M, Janz N, Alderman A, Graff J, et al. Patient-Reported Quality of Life and Satisfaction with Cosmetic Outcomes After Breast Conservation and Mastectomy with and without Reconstruction: Results of a Survey of Breast Cancer Survivors. Ann Surg. 2015;261(6):1198-206.

105. van Bommel ACM, Schreuder K, Veenstra RK, de Ligt KM, MTFD VP, Maduro JH, et al. Discrepancies Between Surgical Oncologists and Plastic Surgeons in Patient Information Provision and Personal Opinions Towards Immediate Breast Reconstruction. Ann Plast Surg. 2018.

106. Festinger L. Conflict, Decision, and Dissonance. Stanford, CA: Stanford University Press; 1964.

107. Blome C, Augustin M. Measuring Change in Quality of Life: Bias in Prospective and Retrospective Evaluation. Value in Health. 2015;18:110-5.

108. Berlin NL, Hamill JB, Qi J, Kim HM, Pusic AL, Wilkins EG. Nonresponse bias in survey research: lessons from a prospective study of breast reconstruction. Journal of Surgical Research. 2018;224:112-20.

109. de Rooij BH, Ezendam NPM, Mols F, Vissers PAJ, Thong MSY, Vlooswijk CCP, et al. Cancer survivors not participating in observational patient-reported outcome studies have a lower survival compared to participants: the population-based PROFILES registry. Qual Life Res. 2018;27(12):3313-24.

110. Hutchings A, Grosse Frie K, Neuburger J, van der Meulen J, Black N. Late response to patient-reported outcome questionnaires after surgery was associated with worse outcome. J Clin Epidemiol. 2013;66(2):21825.

111. Dunsch F, Evans DK, Macis M, Wang Q. Bias in patient satisfaction surveys: a threat to measuring healthcare quality. BMJ Glob Health. 2018;3(e000694).

112. Charles C, Gafni A, Freeman E. Implementing shared treatment decision making and treatment decision aids: a cautionary tale. Psicooncologia. 2010;7(2-3):243-55.

113. Rothman ML, Beltran P, Cappelleri JC, Lipscomb J, Teschendorf B. Patient-Reported Outcomes: Conceptual Issues. Value in Health. 2007;10:S66-S75.

114. Netherlands Comprehensive Cancer Organisation. Oncoguide [Available from: www.oncoguide.nl.

115. Sun CS, Cantor SB, Reece GP, Fingeret MC, Crosby MA, Markey MK. Helping patients make choices about breast reconstruction: A decision analysis approach. Plast Reconstr Surg. 2014;134(4):597-608.

116. Murawa P, Murawa D, Adamczyk A, Połom K. Breast cancer: Actual methods of treatment and future trends. Reports of Practical Oncology and Radiotherapy. 2014;19:165-72.

117. van Bommel ACM, Spronk PER, Mureau MAM, Siesling S, Smorenburg CH, Tollenaar R, et al. Breast-ContourPreserving Procedure as a Multidisciplinary Parameter of Esthetic Outcome in Breast Cancer Treatment in The Netherlands. Annals of Surgical Oncology. 2019;26(6):1704-11.

118. Spronk PER, Volders J, van den Tol P, Smorenburg CH, Vrancken Peeters MTFD. Breast conserving therapy after neoadjuvant chemotherapy; data from the Dutch Breast Cancer Audit. European Journal of Surgical Oncology. 2019;45(2):110-7.

119. MacNeill F, Karakatsanis A. Over surgery in breast cancer. The Breast. 2017;31:284-9.

120. The Council for Public Health and Society. No evidence without context: About the illusion of evidencebased practice in heatchcare. Den Haag2017.

121. Cribb A, Owens J. Whatever suits you: unpicking personalization for the NHS. Journal of Evaluation in Clinical Practise. 2010;16:310-4.

122. Bos W. Stop met wegduiken voor pijnlijke keuzes in de zorg, bepleit Wouter Bos. De Volkskrant. 2018 Oct 12, 2018. 


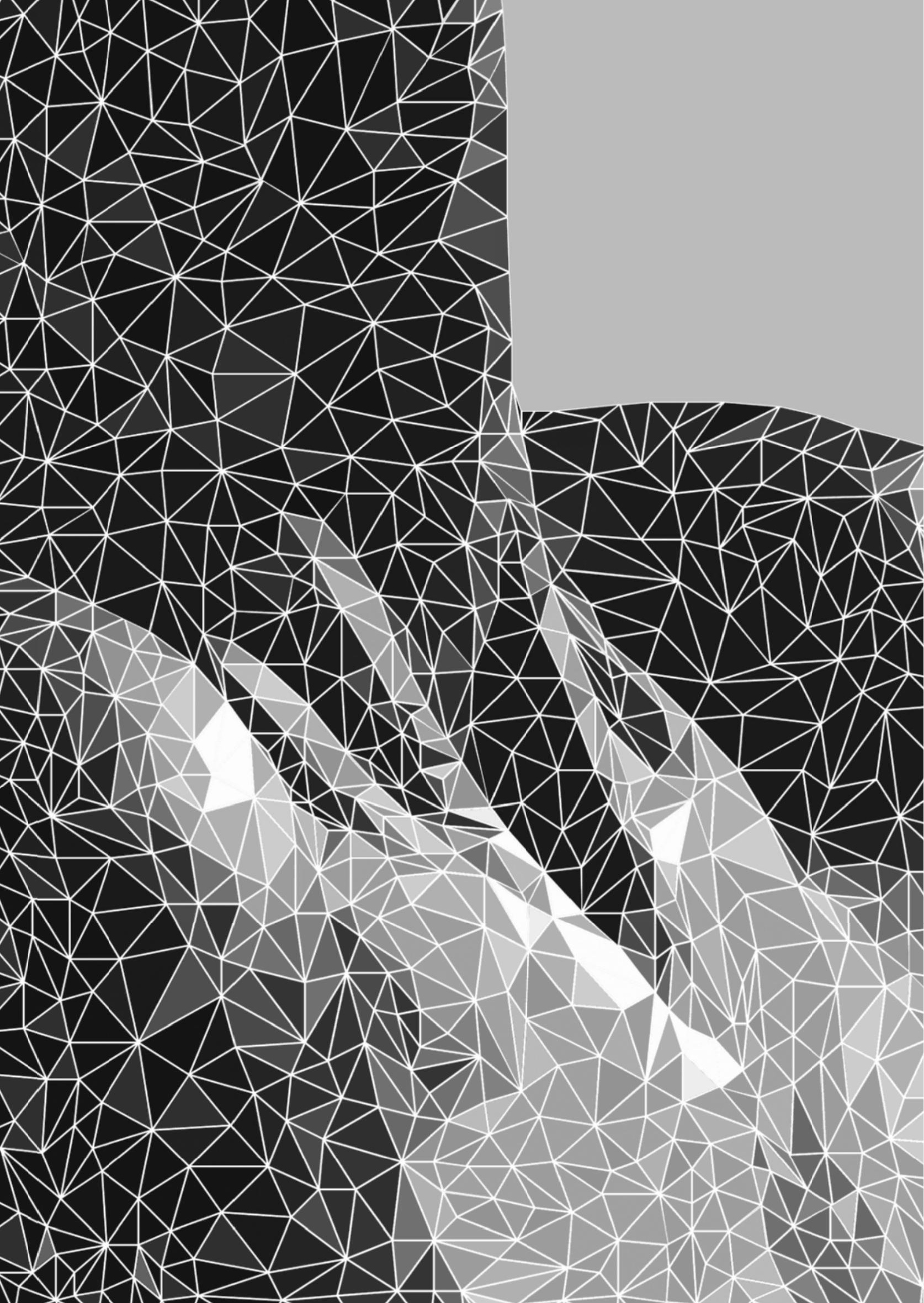


NEDERLANDSE SAMENVATTING

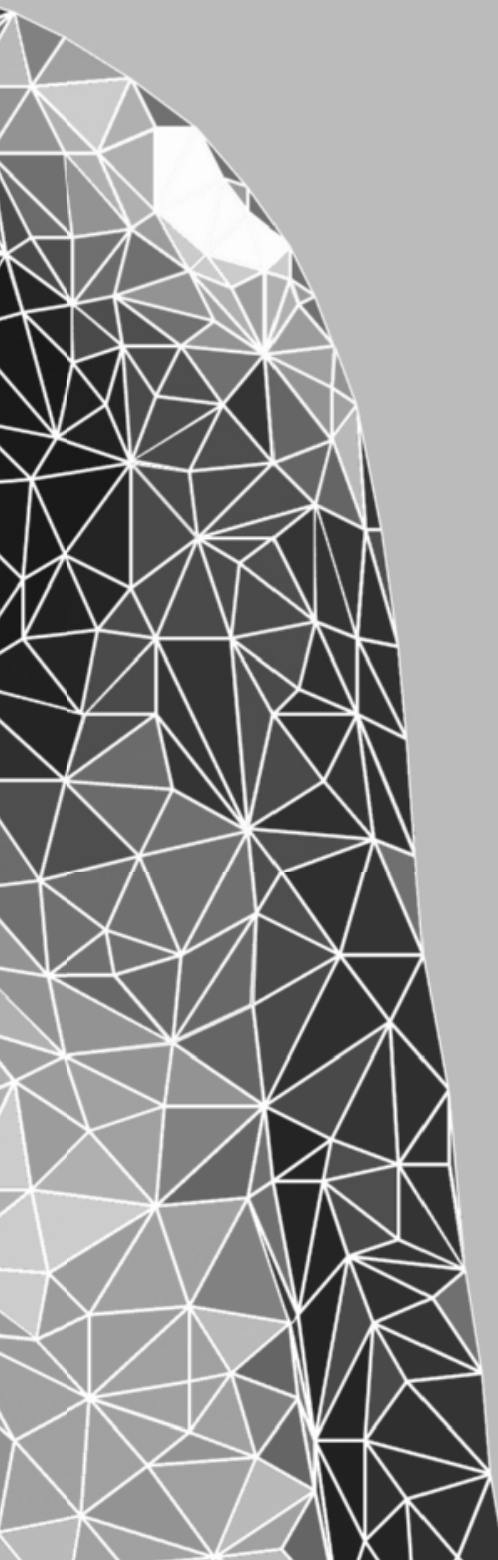





\section{Nederlandse samenvatting}

Borstkanker is wereldwijd de meest gediagnosticeerde vorm van kanker en de belangrijkste doodsoorzaak onder vrouwen. De behandeling van borstkanker bestaat uit chirurgische verwijdering van het aangedane borstweefsel (borstsparende chirurgie) of de gehele aangedane borst (mastectomie of borstamputatie). Aanvullend op een mastectomie kan een borstreconstructie uitgevoerd worden, om zo de vorm van de borst te herstellen. Daarnaast wordt borstkanker locoregionaal behandeld met radiotherapie, of systemisch met chemotherapie, antihormonale therapie, of doelgerichte therapie. Na curatieve behandeling van borstkanker wordt de gezondheid van patiënten ten minste vijf jaar gevolgd tijdens de nazorg. Deze nazorg bestaat uit een jaarlijks mammogram ter detectie van recidiverende kanker (de nacontrole), en consulten ter ondersteuning van de fysieke en psychosociale gezondheid van de patiënt. De aanbevelingen voor behandeling en nazorg zijn in Nederland beschreven in de NABON (Nationaal Borstkanker Overleg Nederland) multidisciplinaire richtlijn voor borstkanker.

Per patiënt wordt, in samenspraak tussen de arts en patiënt, bepaald welke combinatie van behandelmodaliteiten wordt toegepast om de ziekte te cureren. Behandelmodaliteiten hebben namelijk hun eigen voor- en nadelen: de voordelen ten aanzien van het bestrijden van de kanker, en de eventuele nadelen van het ondergaan van de behandeling en de effecten van deze behandeling op de gezondheid en kwaliteit van leven van de patiënt. Tevens hebben alle behandelingen hun eigen (directe) bijwerkingen en/of effecten die later na de behandeling optreden. Wanneer meerdere (behandel)opties voorhanden zijn, kan er variatie tussen ziekenhuizen of patiëntgroepen ontstaan in de toepassing ervan. Enerzijds kan variatie een aanwijzing zijn voor keuzen die in lijn zijn met de wensen en voorkeuren van de individuele patiënt. Anderzijds kan variatie een aanwijzing zijn voor ongelijke toegang tot of kwaliteit van de zorg.

\section{Gepersonaliseerde borstkankerzorg: variatie in behandeling en nazorg vanuit een patiëntperspectief}

Dit proefschrift richt zich op aspecten van variatie in de borstkankerzorg in Nederland, gezien vanuit het perspectief van de patiënt. Het doel van dit proefschrift was om na te gaan in hoeverre voorkeuren en behoeften van patiënten en hun ervaringen met informatievoorziening en gedeelde besluitvorming, de geobserveerde variatie in gebruik van borstkankerzorg in Nederland kan verklaren. Dit werd uitgewerkt in drie delen. Het eerste deel onderzoekt verklaringen voor de variatie in timing van chemotherapie, dat wil zeggen, voor of na de operatieve verwijdering van de borst of het borstweefsel. Het tweede deel behandelt de variatie geobserveerd voor de toepassing van directe borstreconstructie bij patiënten behandeld met een mastectomie. Het derde deel beschouwt de mogelijkheden voor het verder personaliseren van de nazorg voor borstkanker. 


\section{Eerste deel: besluitvorming over de timing van chemotherapie}

Chemotherapie kan preoperatief worden ingezet, ook wel neoadjuvant, met als voordelen de tumor te verkleinen en zo borstsparende en okselsparende chirurgie mogelijk te maken, en om de response van de tumor op de chemotherapie te bepalen. Toch is postoperatieve, ook wel adjuvante, toepassing nog steeds de gebruikelijke timing van chemotherapie, en werd een grote variatie tussen ziekenhuizen in Nederland gezien in toepassing van neoadjuvante chemotherapie. Deze variatie in de timing van chemotherapie voor borstkanker werd onderzocht vanuit zowel het perspectief van de patiënt in Hoofdstuk 1, als het perspectief van de behandelaar in Hoofdstuk 2. Op basis van de ervaringen van 400 borstkankerpatiënten behandeld met chemotherapie vonden we dat slechts een minderheid (stadium II: $14 \%$, stadium III: $31 \%$ ) van de patiënten behandeld met adjuvante chemotherapie voorafgaand aan de behandeling geïnformeerd was over de mogelijkheid van neoadjuvante chemotherapie. Tevens vonden we een aanzienlijke variatie in klinische opinies over de toepassing van neoadjuvante chemotherapie bij zowel chirurgen als medisch oncologen. Dit is mogelijk een verklaring voor het gebrek aan informatievoorziening over neoadjuvante chemotherapie.

\section{Tweede deel: besluitvorming over borstreconstructie}

Een borstreconstructie kan worden toegepast bij patiënten behandeld met een mastectomie om de contour of vorm van de borst te herstellen. Hoofdstuk $\mathbf{3}$ beschrijft de toegevoegde waarde van reconstructieve chirurgie, maar ook van borstsparende chirurgie, op de kwaliteit van leven van de borstkankerpatiënt ten opzichte van behandeling met (alleen) mastectomie. Daarbij hadden borstsparende chirurgie en de verschillende reconstructieve technieken hun eigen voordelen. De kennis over deze voordelen kan gebruikt worden in de afstemming van de wens van de patiënt en het uiteindelijke resultaat. Toch werd er variatie gezien tussen ziekenhuizen in de toepassing van directe borstreconstructies, en was het gemiddelde percentage borstreconstructies laag. In Hoofdstuk 4 worden de ervaringen met informatievoorziening over borstreconstructie van ruim 500 patiënten beschreven, vanuit de hypothese dat dit mogelijk de geobserveerde variatie kan verklaren. Ongeveer $80 \%$ van de patiënten onderging een chirurgische ingreep conform hun wensen en behoeften. De toepassing van directe borstreconstructie lijkt daarmee grotendeels afgestemd op de individuele patiënt. We vonden echter ook dat significant minder patiënten die geen borstreconstructie ondergingen (ongeveer drie-vierde) geïnformeerd waren over de optie tot borstreconstructie ten opzichte van patiënten die wel een borstreconstructie ondergingen (nagenoeg 100\%). Er is dus ruimte voor verbetering van deze informatievoorziening. Dit is belangrijk, omdat we tevens vonden dat deze informatie de kans op een borstreconstructie met 14 keer vergrootte. 


\section{Derde deel: mogelijkheden voor het personaliseren van nazorg}

In het laatste deel van dit proefschrift onderzochten we de mogelijkheden voor het verder personaliseren van de nazorg voor borstkanker. In Hoofdstuk 5 en Hoofdstuk 6 inventariseerden we de ervaringen van 400 borstkankerpatiënten die 1 tot 5 jaar geleden behandeld werden voor borstkanker. Zij rapporteerden zowel hun gezondheidsklachten als hun kwaliteit van leven. Nagenoeg iedere patiënt rapporteerde gezondheidsklachten (93\%), waarbij meer dan de helft last had van vermoeidheid, psychologische klachten, klachten in het borstgebied, musculoskeletale klachten, en klachten van het zenuwstelsel of reproductieve systeem. Vooral behandeling met chemotherapie en anti-hormonale therapie waren geassocieerd met het rapporteren van klachten. Daarbij hadden bijna alle klachten een effect op het functioneren van deze patiënten, maar met name vermoeidheid, musculoskeletale klachten, gastro-intestinale klachten, en klachten van het zenuwstelsel hadden een effect op meerdere domeinen van functioneren. Op basis van 41 studies gevonden in onze literatuurstudie beschrijft Hoofdstuk $\mathbf{7}$ de patiëntvoorkeuren en betrokkenheid in keuzen over nazorg bij borstkanker. Hoewel patiënten al erg betrokken werden in keuzen over (uitgestelde) borstreconstructie, was er weinig bewijs dat zorgverleners patiënten betrokken in keuzen over anti-hormonale therapie, menopauzale klachten, en de inrichting van nazorg. Dat terwijl patiënten hier wel voorkeuren en wensen over uitten. We zien dus mogelijkheden om zorg verder te personaliseren, door patiënten beter te betrekken bij deze keuzen. 


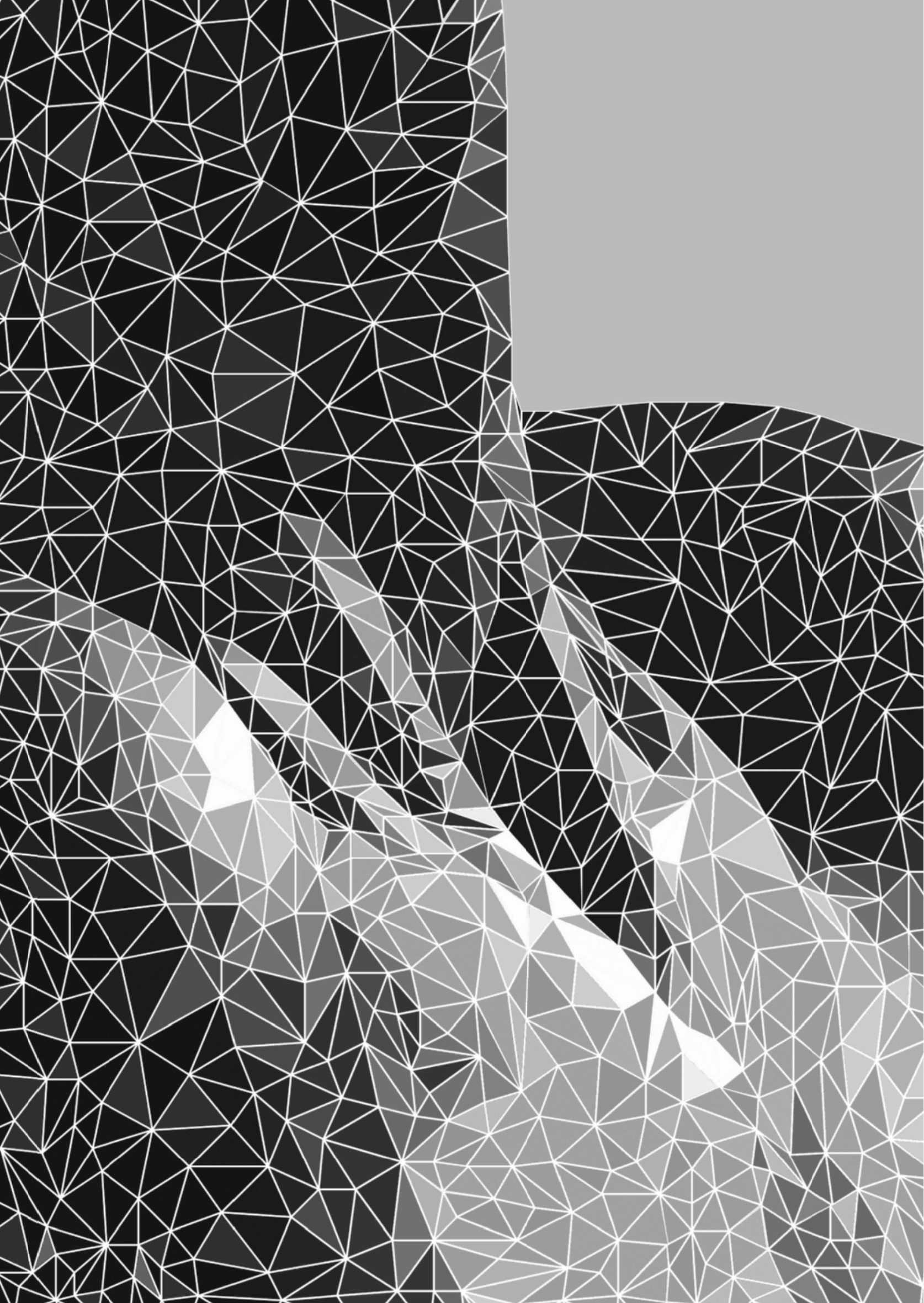


LIST OF PUBLICATIONS

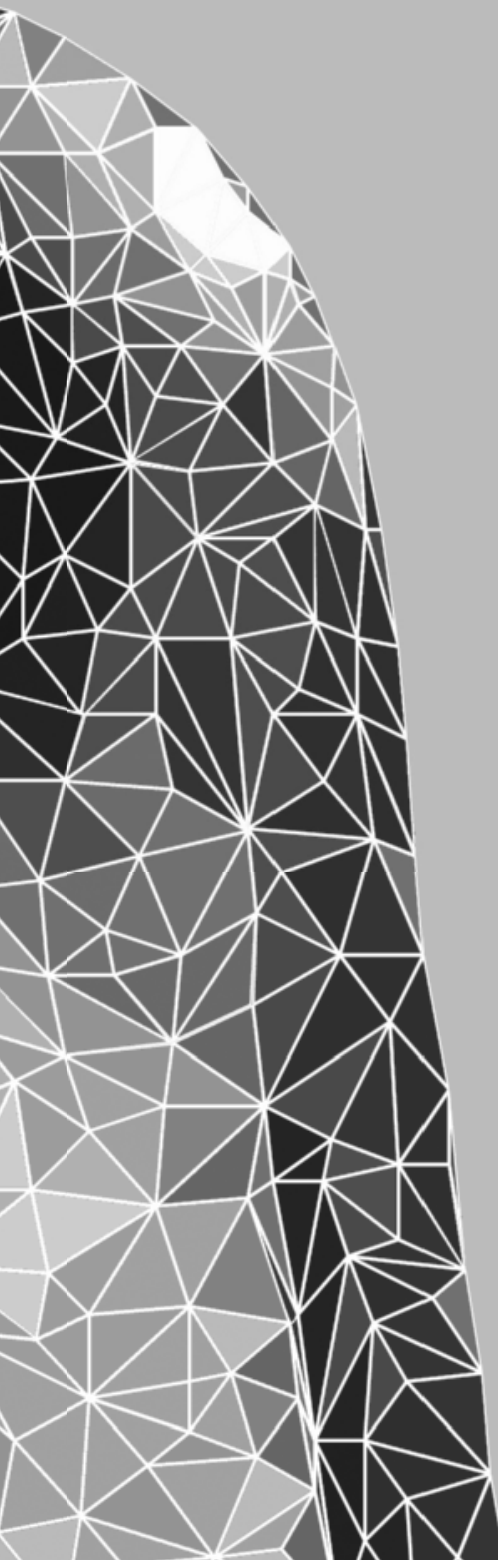





\section{In this thesis}

De Ligt KM, Heins M, Verloop J, Ezendam NPM, Smorenburg CH, Korevaar JC, Siesling S. The impact of health symptoms on quality of life in breast cancer survivors. Submitted at Cancer, June 2019.

Kouwenberg CAE, de Ligt KM, Kranenburg LW, Rakhorst $\mathrm{H}$, de Leeuw D, Siesling S, Busschbach JJ, Mureau MAM. Long-term health-related quality of life after four common surgical treatment options for breast cancer and the effect of complications - a retrospective patient-reported survey among 1871 patients. Submitted at Plastic and Reconstructive Surgery, May 2019.

De Ligt KM, van Egdom LSE, Koppert LB, Siesling S, van Til JA. Opportunities for personalised followup care among patients with breast cancer: A scoping review to identify preference-sensitive decisions. Eur J Cancer Care (Engl). 2019 May;28(3):e13092. doi: 10.1111/ecc.13092.

De Ligt KM, Heins M, Verloop J, Smorenburg CH, Korevaar JC, Siesling S. Patient-reported health problems and healthcare use after treatment for early-stage breast cancer. Breast. $2019 \mathrm{Apr}$ 4;46:4-11. doi: 10.1016/j.breast.2019.03.010.

Spronk PER, de Ligt KM, van Bommel ACM, Vrancken Peeters MTFD, Siesling S. Current decisions on neoadjuvant chemotherapy for early breast cancer: Experts' experiences in the Netherlands. Patient Education and Counselling. Jul 2018. DOI: 10.1016/j.pec.2018.07.012

De Ligt KM, van Bommel ACM, Schreuder K, Schrieks M, Maduro JH, Baas-Vrancken Peeters MTFD, Mureau MAM, Siesling $S$. The effect of being informed on receiving immediate breast reconstruction in breast cancer patients. European Journal of Surgical Oncology. May 2018, 44(5); p. 717-724. DOI: https://doi.org/10.1016/j.ejso.2018.01.226

De Ligt KM, Spronk PER, van Bommel ACM, Vrancken Peeters MTFD, Siesling S. Patients' experiences with decisions on timing of chemotherapy for breast cancer. The Breast 37 (2018) 99e106

\section{Other publications}

Van Bommel ACM, Scheuder K, Veenstra RK, de Ligt KM, Vrancken Peeters MJTFD, Maduro JH, Siesling S, Mureau MAM. Discrepancies Between Surgical Oncologists and Plastic Surgeons in Patient Information Provision and Personal Opinions Towards Immediate Breast Reconstruction. Annals of Plastic Surgery. Jul 2018, 81(4). DOI: 10.1097/SAP.0000000000001572

Ruiz A, van Hillegersberg R, Siesling S, Castro-Benitez C, Sebagh M, Wicherts DA, de Ligt KM, Goense L, Giacchetti S, Castaing D, Morère J, Adam R. Surgical resection versus systemic therapy for breast cancer liver metastases: Results of a European case matched comparison. European Journal of Cancer. 2018;95:1-10. DOI: https://doi.org/10.1016/j.ejca.2018.02.024. 
Schreuder K, van Bommel ACM, de Ligt KM, Maduro JH, Baas-Vrancken Peeters MTFD, Mureau MAM, Siesling S. Hospital factors explaining the variation in immediate post mastectomy breast reconstruction for breast cancer in the Netherlands. The Breast. 2017 Aug;34:96-102. doi: 10.1016/j.breast.2017.05.011. Epub 2017 May 25

De Ligt KM, Witteveen A, Siesling S, Steuten LGM. Shifting breast cancer surveillance from current hospital setting to a community based setting: A cost-effectiveness study. BMC Cancer, 18(1). 2018. DOI: 10.1186/s12885-018-3992-7

\section{Books and reports}

Veldhuis M, Ezendam E, De Ligt KM, Oerlemans S. Kankerzorg in Beeld: (Over)leven met en na kanker. April 2019. ISBN 978-90-72175-49-6.

De Ligt KM, Bretveld RW, Bastiaannet E, Liefers GJ, Smorenburg CH, van der Sangen MJC, Siesling S. Kankerzorg in Beeld: De oudere patiënt (hoofdstuk borstkanker). Dec 2016. ISBN 978-90-7217545-8.

\section{Abstracts}

\section{Oral presentations}

De Ligt KM, Heins M, Verloop J, Ezendam NJP, Smorenburg CH, Korevaar JC, Siesling S. Patientreported health problems and healthcare use after treatment for early-stage breast cancer and the impact of health problems on quality of life in breast cancer survivors. Presented at Bossche Mammacongres, Sint-Michelsgestel, June 2019.

De Ligt KM, Spronk PER, van Bommel ACM, Vrancken Peeters MTDF, Siesling S, Smorenburg CH. Patients' Experiences with Decisions on Timing of Chemotherapy for Breast Cancer. Presented at IACR 2017, October 2017.

De Ligt KM, Spronk PER, van Bommel ACM, Vrancken Peeters MTDF, Siesling S, Smorenburg CH. Patient experiences with shared decision-making on neoadjuvant chemotherapy in early breast cancer. Presented at Bossche Mammadagen, Sint-Michelsgestel, 2017, June 2017.

De Ligt KM, Witteveen A, Siesling S, Steuten LMG. Cost-effectiveness analysis of an alternative follow-up strategy for breast cancer patients that have received breast conserving treatment: referral to the National Screening Programme after one year of regular hospital follow-up compared to regular five-year follow-up at the hospital. Presented at EBCC10, Amsterdam, March 2016. 


\section{Poster presentations}

De Ligt KM, Heins M, Verloop J, Smorenburg CH, Korevaar JC, Siesling S. Patient-reported health problems and healthcare use after treatment for early-stage breast cancer. Presented at ESMO Breast, Berlin 2019.

De Ligt KM, Spronk PER, van Bommel ACM, Vrancken Peeters MTDF, Siesling S, Smorenburg CH. Patient experiences with shared decision-making on neoadjuvant chemotherapy in early breast cancer. Presented at WEON 2017, Antwerp, June 2017.

De Ligt KM, Bretveld R, Smorenburg CH, Bastiaannet E, Lieffers GJ, van der Sangen MJC, Siesling S. Breast cancer care for older patients in the Netherlands: a population based study 2005-2013. Presented at EBCC10, Amsterdam, March 2016.

De Ligt KM, van Bommel ACM, Mureau MAM, Schreuder K, Vrancken Peeters MTDF, Schrieks MA, Maduro JH, Siesling S. Patient reported shared decision making regarding breast reconstruction after mastectomy for invasive breast cancer. Presented at EBCC10, Amsterdam, March 2016. 


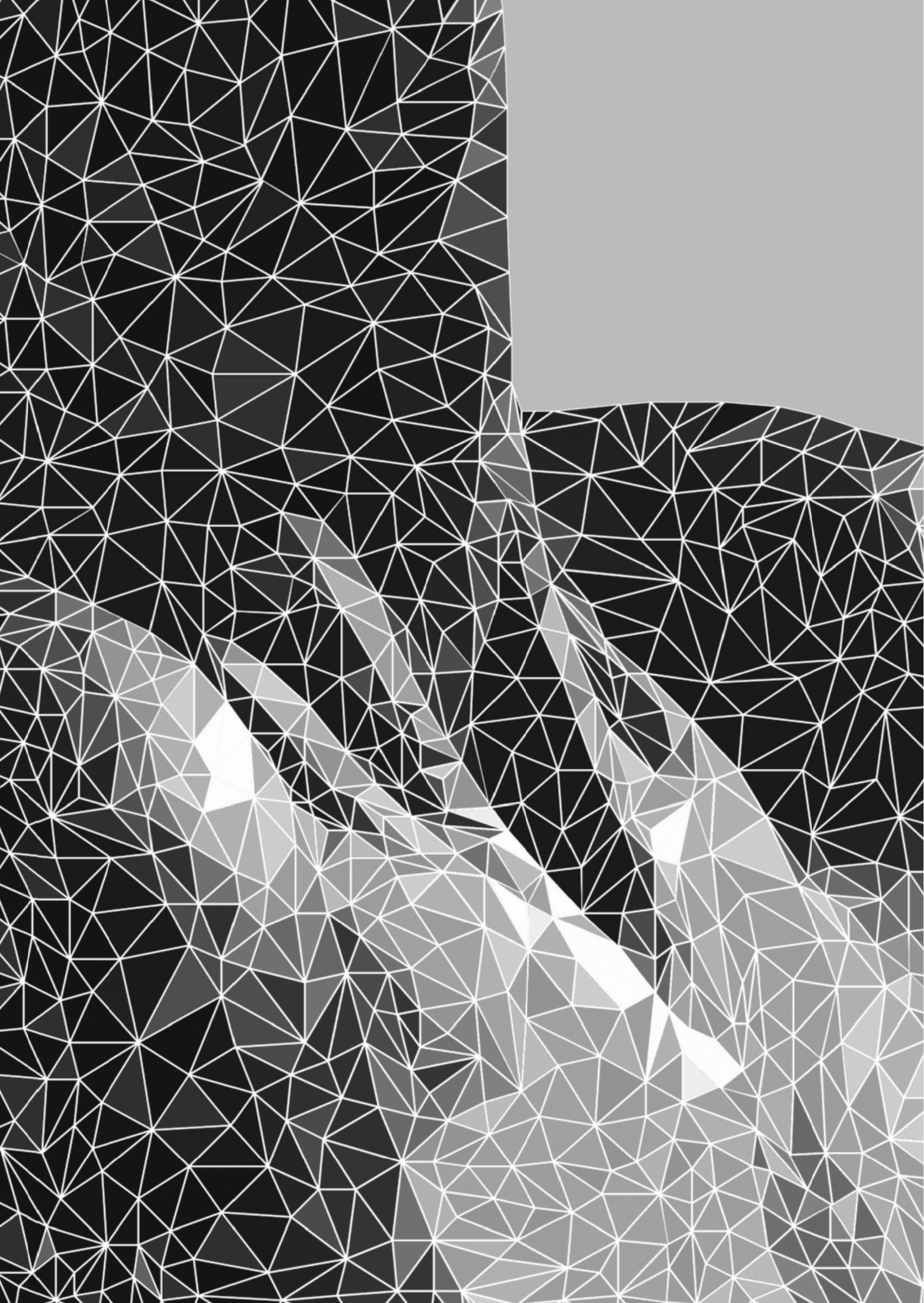


DANKWOORD

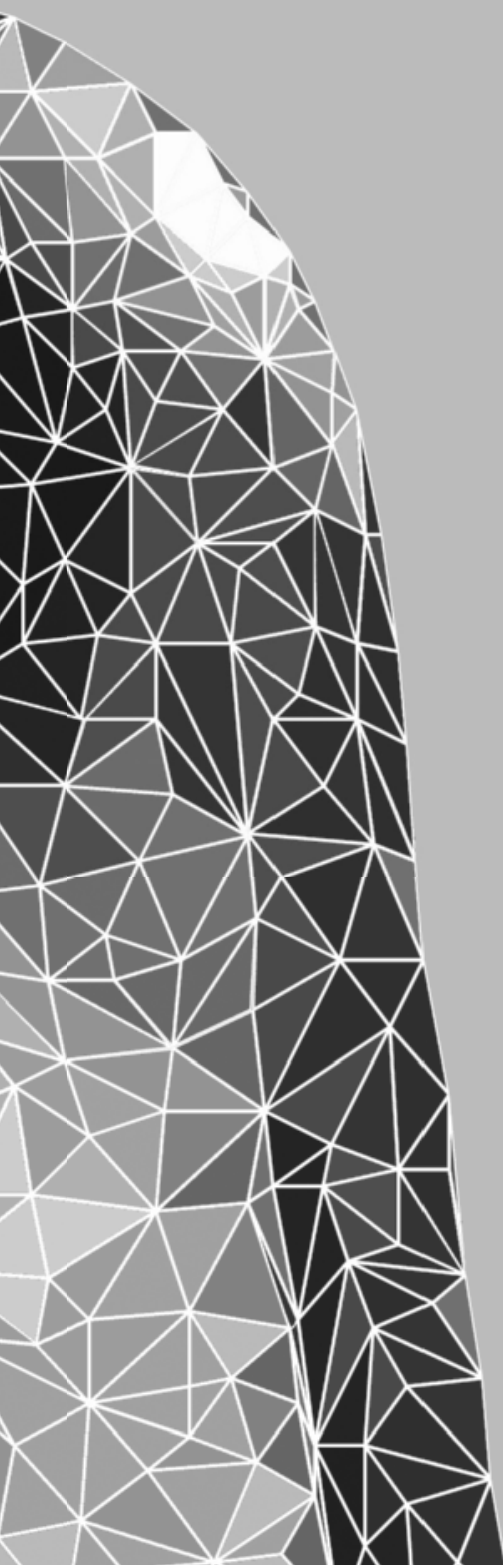





\section{Dankwoord}

Negenentwintig augustus 2014 was een zomerse dag. Ik bracht mijn laatste uurtjes als Masterstudent Gezondheidswetenschappen door in een zaaltje op de campus van de Universiteit Twente (UT), waar ik een presentatie gaf naar aanleiding van mijn stageperiode bij Integraal Kankercentrum Nederland (IKNL). Half september zou ik bij IKNL beginnen als onderzoeksassistent, een baan die mijn stagebegeleider Sabine me had aangeboden. Ik was niet overdreven geïnteresseerd in onderzoek doen, maar had wel interesse gekregen voor de wereld van de oncologie.

Vijf jaar na die middag in dat zaaltje, zijn we in een iets groter zaaltje aan de UT, en geef ik weer een presentatie ter afronding van een periode bij IKNL. Die van mijn promotie! Ik wil graag degenen bedanken die hebben bijgedragen aan mijn proefschrift en het onderzoek in dit proefschrift.

Allereerst gaat mijn dank uit naar mijn begeleidingscommissie, bestaande uit mijn promotor Prof. dr. Sabine Siesling, Dr. Marc Mureau, en Dr. Carolien Smorenburg. Sabine, wat ben ik blij dat ik destijds de kans van je kreeg om bij IKNL te beginnen. Toen ik kort geleden tijdens een overleg zei dat ik 'zin had om met de data aan de slag te gaan', moesten we allebei lachen. Want wie had dat uiteindelijk gedacht? Ik kijk terug op jaren waar ik niet alleen leerde over onderzoek doen en statistiek, maar daar ook steeds meer plezier in kreeg. Bedankt voor de tijd en ruimte die ik kreeg om mij persoonlijk te ontwikkelen. Soms ging dat (ook door de omstandigheden) met één stap vooruit en dan weer twee terug, maar mede dankzij jouw positieve instelling en de humor die we samen delen, werd ook daar een mooie draai aan gegeven. Ik bewonder je energie en je doorzettingsvermogen!

Marc en Carolien, jullie haakten ongeveer halverwege mijn promotietraject aan als mijn promotieteam. Ik had als onderzoeksassistent bij jullie beide in een project meegewerkt en wilde de onderwerpen uit die projecten graag met jullie verder uitwerken. Marc, de manier waarop jij snel kan schakelen en knopen kan doorhakken gaven enorm richting aan mijn promotietraject. Bedankt daarvoor! Tegelijk was je ook van de details, want menig manuscript werd voorzien van punten en komma's en prachtige Engelse woorden. Carolien, je kon mij er goed op wijzen dat het mijn proefschrift is (en ik dus mag beslissen ;-) ) en dat er nog meer belangrijke dingen zijn naast promoveren. Bedankt voor je eeuwige aanmoediging, maar ook voor de adviezen als het even niet zo lekker liep.

De hoofdstukken in dit proefschrift komen onder andere voort uit samenwerkingen met andere (junior) onderzoekers, arts-onderzoekers, en medisch specialisten. In het bijzonder: Annelotte, Casimir, Marianne, Pauline, en Pien. Het was fijn om met jullie samen te werken en ik ben trots op de artikelen die we maakten. Alle andere co-auteurs, namelijk Jan van Busschbach, Nicole Ezendam, Linetta Koppert, Joke Korevaar, Leonieke Kranenburg, Daniëlle de Leeuw, John Maduro, Hinne Rakhorst, Janine van Til, en Marie Jeanne Vrancken Peeters, bedankt voor al jullie input op de manuscripten. Samen kom je verder. 
Ik wil de leden van de beoordelingscommissie, Prof. dr. W. H. van Harten, Dr. C.H.C. Drossaert, Prof. dr. L. van de Poll-Franse, Prof. dr. J.A. Hazelzet, Prof. dr. S.C. Linn, en Prof. dr. J.H.G. Klinkenbijl bedanken voor het kritisch lezen en beoordelen van het manuscript en hun bereidheid om deel te nemen aan de oppositie.

Voorafgaand aan mijn promotieplechtigheid is een symposium georganiseerd over het 'Ondersteunen van patiënten met kanker: Informeren, kiezen, monitoren, empoweren!'. Voor de totstandkoming van dit symposium wil ik graag Ellen van der Blom-Wirosoewignjo, Henk Hummel, Ingrid de Kaste-Krisman, Linda Bergmans, Marlon Tonis, en Jan Maarten van der Zwan bedanken. Het maakt deze dag nog net iets meer bijzonder.

Mammaonderzoekers en andere graaggeziene bezoekers van het teamoverleg op vrijdag: wat zijn wij de afgelopen jaren een leuk team geworden! Janneke, Jolanda, Linda, Marissa, Kay, Marianne, Rianne, Ingrid, Sander, Adri, Irma, en Jaap: bedankt voor de fijne traktaties, teamuitjes, borrels, (promotie)feestjes, sinterklaasdobbels, schrijfweek, en congressen waar we samen geweest zijn! In iets breder kader wil ik ook de leden van het team Borstkanker bedanken voor hun fijne samenwerking.

Lieve collega's van Onderzoek en van locatie Utrecht, namelijk Annemarie, Anke, Amanda, Avinash, Carla, Dorien, Heidi, Jan Maarten, Lilian, Marianne, Maite, Mirian, Mujde, Natasja, Nora, Robin, Willemieke, en alle anderen. Het is niet altijd handig om koffiepauze-lunchpauze-koffiepauze te houden en dan ook nog een rondje te gaan lopen, maar wel heel gezellig op de soms hele lange dagen! Amanda en Mirian, bij deze vraag ik officieel een plekje op de Onderzoek Wall of Fame aan voor de kaft van mijn proefschrift;-)

Collega's uit Enschede, bij jullie begon het allemaal. Enschede is nog altijd een beetje thuiskomen.

In het kader van een ander mooi project afgelopen jaar, eveneens met een boekje als resultaat, wil ik graag Nicole Ezendam, Simone Oerlemans, en Miranda Velthuis bedanken. Ik denk dat we een Kankerzorg in Beeld-rapport hebben afgeleverd waar we trots op mogen zijn!

Hoewel ik het grootste deel van mijn tijd doorbracht op kantoor bij IKNL, sloot ik eens in de twee weken aan bij de vakgroepoverleggen van de vakgroep Health Technology and Services Research. Ondanks dat het in het begin een beetje vreemd voelde dat een groot deel van je docenten ineens je directe collega's zijn, vond ik het leuk om in jullie groep opgenomen te worden. Bedankt daarvoor! In het bijzonder wil ik Annemieke Witteveen bedanken: ergens tijdens mijn stage ben ik onderzoek toch als optie gaan zien, en ik weet zeker dat jij daar aan bijgedragen hebt ;-).

De laatste loodjes zijn het meest chaotisch. Kay, Melinda, Marissa, Marianne, Willemieke, fijn dat jullie mijn (concept)proefschrift rustig hebben nagelezen. Wie uiteraard de meeste orde in deze chaos schiepen, waren mijn paranimfen Marissa en Aniek, en Aimée als uitstekende achterwacht tijdens de zomervakantie. Ik vind het een eer dat jullie me met raad en daad bijstaan, zowel op de dag van de verdediging als tijdens de aanloop hier naartoe. 
Deze onderzoeken hadden niet kunnen bestaan zonder de onderliggende data. Ik wil graag alle deelnemers aan de vragenlijsten bedanken voor het delen van hun ervaringen. Verder wil ik de medewerkers van PROFIEL bedanken voor hun hulp bij het opzetten en uitvoeren van de dataverzamelingen. In het bijzonder dank aan Nicole Horevoorts: fijn dat jij altijd een antwoord hebt op mijn vragen! Ook wil ik graag de datamanagers van de Nederlandse Kankerregistratie bedanken. Nu ik zelf een lesje dataverzameling heb gekregen, waardeer ik jullie inzet, precisie, en doorzettingsvermogen nog veel meer.

Er zijn dingen waar je aan moet wennen. Vaak zijn dat wendingen. Tot slot wil ik graag mijn familie en vrienden bedanken. Voor hun oprechte interesse, hun vriendschap en steun wanneer werk door alle wendingen (van met name afgelopen jaar) als complete bijzaak voelde. Een paar van hen wil ik in het bijzonder bedanken. Lieve opa en oma, jullie steken nooit onder stoelen of banken hoe trots jullie op me zijn en dat geeft me moed om iedere keer weer een stapje verder te gaan. Jullie zijn waarschijnlijk mijn allergrootste fans (en wat was je er graag bij geweest op deze bijzondere dag, oma!). Lieve Ellen, bedankt dat ik een beetje van jouw creatieve brein mocht lenen bij het bedenken van een omslag voor mijn proefschrift. Lieve pap en mam, hoewel het lijkt alsof school, studeren, en promoveren me moeiteloos afgaan, weten wij inmiddels wel beter. Pap en Debora, de deur staat echt altijd voor me open en dat doet me goed. Mam, ik ben blij dat ik je altijd kan bellen. Ik hou van jullie!

Kelly

juli 2019 


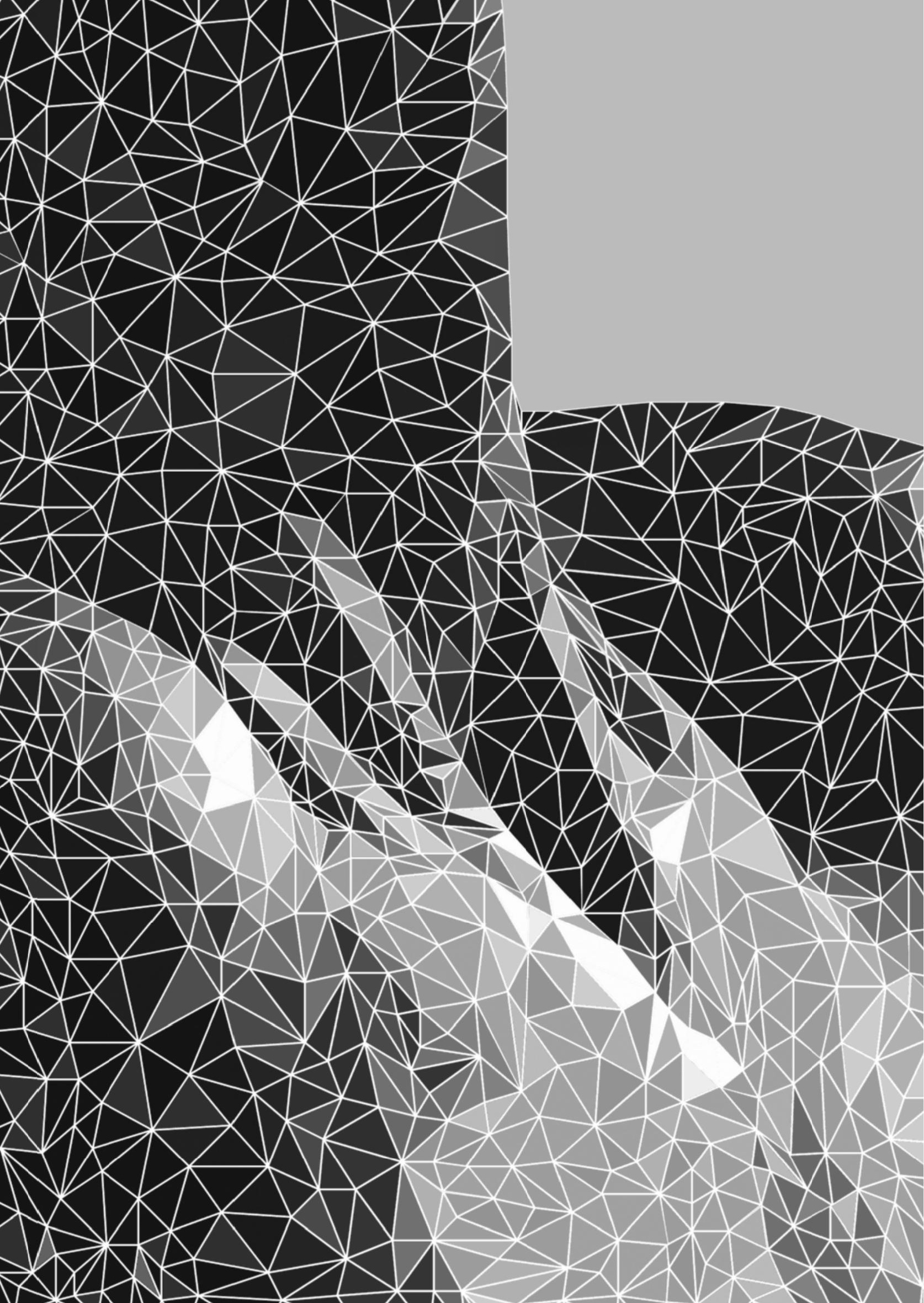


CURRICULUM VITAE

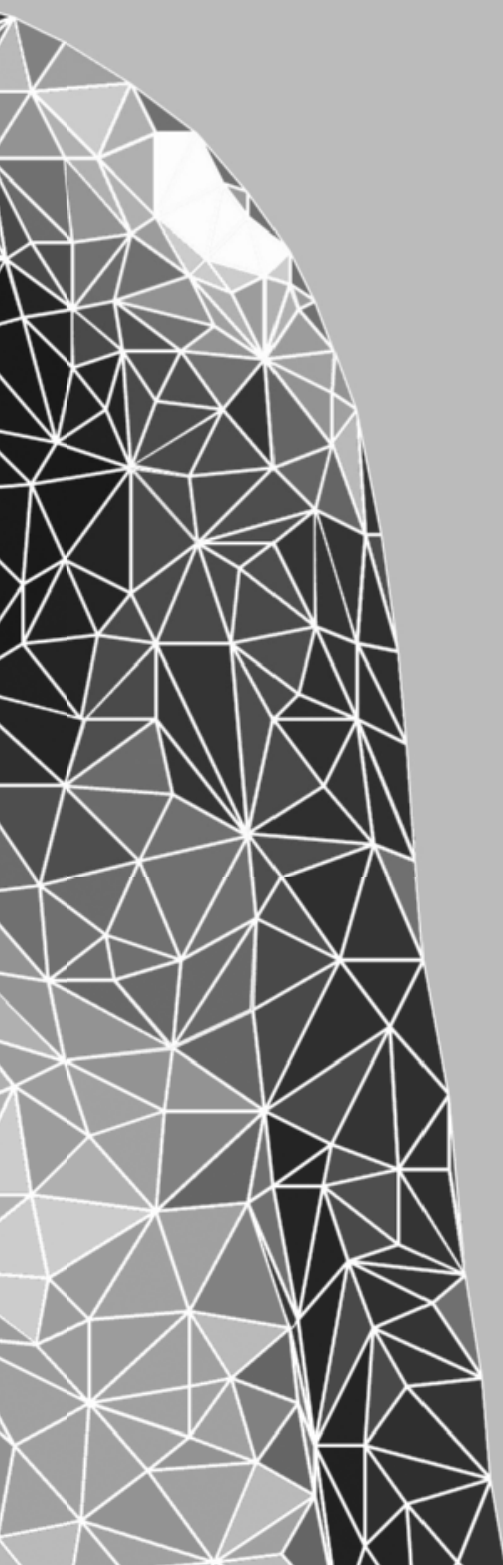





\section{Curriculum Vitae}

Kelly Maureen de Ligt was born on June 25th 1992, in Arnhem, The Netherlands. She attended secondary school at the Liemers College in Zevenaar, which she completed in 2010. In the same year, she started her Bachelor of Health Sciences at the University of Twente, Enschede, and completed this in 2013. Subsequently, she obtained her Masters Degree in Health Sciences at the University of Twente as well. Her final internship during this Master in 2014 was at the Netherlands Comprehensive Cancer Organisation (IKNL), in which she researched the costeffectiveness of breast cancer surveillance care within the setting of the national screening programme. This internship resulted in a job at IKNL as a research assistant, in which she managed the administration of several surveys about breast reconstruction and timing of chemotherapy in breast cancer patients. From 2015 and onwards, Kelly worked as a junior researcher at IKNL, with her main focus still on breast cancer care. This led

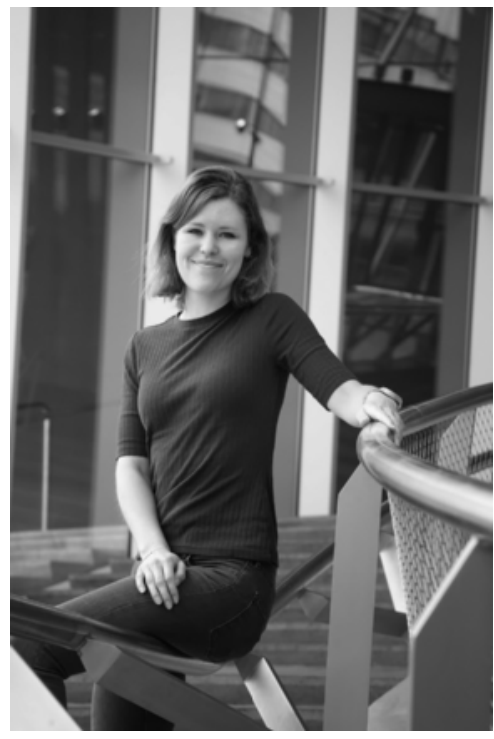
to a position as an external PhD-candidate at the University of Twente, at the Health Technology and Services Research department. The focus of her thesis is on variation in breast cancer care from a patient's perspective. To gain insight in this perspective, she initiated several survey cohorts including large groups of breast cancer patients across the Netherlands. Furthermore, during her PhD, she supervised several Health Sciences students. Within the past year, she worked on the 'Kankerzorg in Beeld' project at IKNL, which led to a report on cancer survivorship in the Netherlands. 




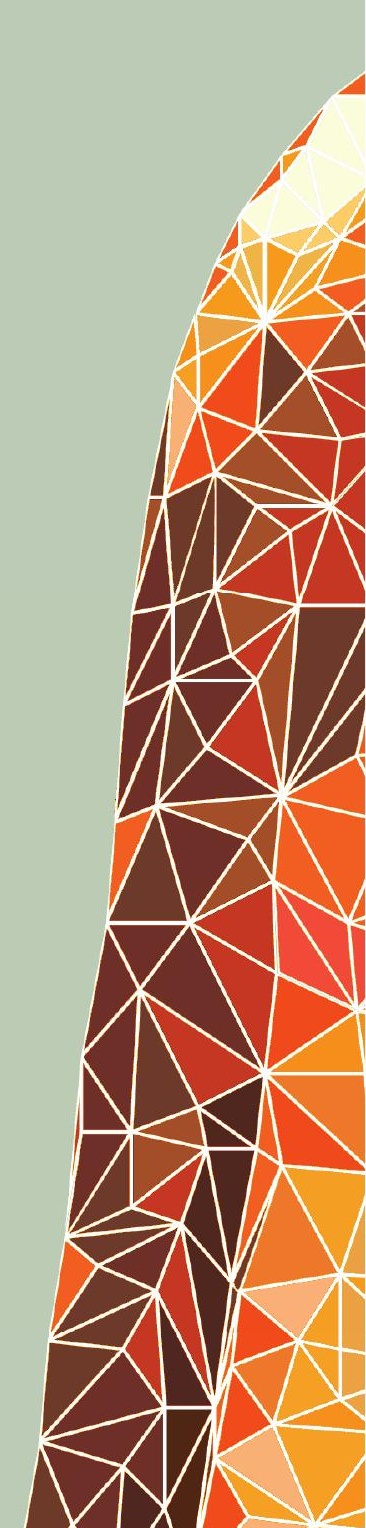

